\title{
Search for Higgs Boson Production in Association with the $W$ Boson in 1.96-TeV Proton-Antiproton Collisions
}

\author{
Yoshio ISHIZAWA
}

September 2005 



\title{
Search for Higgs Boson Production in Association with the $W$ Boson in 1.96-TeV Proton-Antiproton Collisions
}

\author{
Yoshio ISHIZAWA \\ (Doctoral Program in Physics)
}

Submitted to the Graduate School of

Pure and Applied Sciences

in Partial Fulfillment of the Requirements for the Degree of Doctor of Philosophy in Science

at the

University of Tsukuba 



\begin{abstract}
A search for the Standard Model Higgs boson was carried out in $W H \rightarrow \ell \nu b \bar{b}$ process in $p \bar{p}$ collisions at a center of mass energy of $1.96 \mathrm{TeV}$, where $W, H, \ell, \nu, b$ and $p$ denote either a $W^{+}$or $W^{-}$boson, Higgs boson, lepton (electron or muon), neutrino, bottom quark and proton, respectively. The data were collected with the Collider Detector at Fermilab from February 2002 to August 2004. The corresponding integrated luminosity is $319 \mathrm{pb}^{-1}$. We select events containing a single high- $p_{T}$ electron or muon, a large imbalance of the total transverse energy from a neutrino and two $b$ quark jets. The main backgrounds are the $W+$ light flavor/gluon jets and $W+$ heavy flavor jets processes. Requiring the secondary vertex $b$-tagging enables us to reject the $W+$ light flavor/gluon jets events effectively. After all event selections, we observe 187 events which is in agreement with the Standard Model background expectation of $175.2 \pm 26.3$ events, and there is no significant excess originating from the Higgs boson in the reconstructed dijet invariant mass distribution. We thus set a $95 \%$ confidence level upper limit on the production cross section times branching ratio decaying into $b \bar{b}, \sigma(p \bar{p} \rightarrow W H) \times \mathcal{B} r(H \rightarrow b \bar{b})$. The detection efficiency for the $W H$ events, which is necessary for calculating the upper limit, is determined by the Monte Carlo except for the lepton identification efficiency, the lepton trigger efficiency and the $b$-tagging efficiency which are estimated from the CDF real data. The resultant $95 \%$ confidence level upper limits are 10.0 pb to $2.8 \mathrm{pb}$ using at least one $b$-tagging method and $9.7 \mathrm{pb}$ to $6.6 \mathrm{pb}$ using double $b$-tagging method for the Higgs boson mass region $110 \mathrm{GeV} / c^{2}$ to $150 \mathrm{GeV} / c^{2}$, where the Standard Model prediction is approximately one or two order of magnitude lower than the results.
\end{abstract}





\section{Acknowledgments}

I thank Professor Fumihiko Ukegawa and Professor Shinhong Kim with their support throughout my graduate student career. They gave me the opportunity to join the CDF collaboration.

I wish to express my deepest appreciation for my analysis advisers, Wei-Ming Yao and Jason Nielsen, with their devoted support, encouragement and patience. I learned much from their precise ways of thinking and sincere attitudes to physics research. When we found the dijet invariant mass resonance using the $319 \mathrm{pb}^{-1}$ dataset, we spent a hard time to understand the mass resonance. I will not forget their great efforts with crucial points to overcome the hard time, and I could not finish this thesis without their great helps and patience. I am proud of working with them together.

I also greatly appreciate the publication committee members of this $W X \rightarrow \ell \nu b \bar{b}$ analysis, Brian Winer, Oscar Gonzalez-Lopes, Thomas Wright, Beate Heinemann, Song Ming Wang and Thomas Junk. They understood my difficult situation, and held the weekly meeting even during their busy time. In the meeting, they always gave me many great suggestions to understand, bless and publish this analysis.

I would like to gratitude for physics conveners and some people. Beate Heinemann, Song Ming Wang and Stephan Lammel (the exotic physics group), Wei-Ming Yao, John Conway, Aaron Dominguez, Thomas Junk and Anton Anastassov (the higgs working group), Takasumi Maruyama and Evelyn Thomson (the top physics group), Jason Nielsen and Robin Erbacher (the lepton + jets group), Stephen Levy and Thomas Wright (the high- $p_{T} b$-tag group), Florencia Cannelli, Tommaso Dorigo and Anwar Bhatti (the jet energy correction group), Young-Kee Kim and Luciano Ristori (the joint physics group and the spokespersons of the CDF collaboration), Tomonobu Tomura, Christopher Neu, Benjamin Kilminster and Yoshiaki Kusakabe organized my analysis, and they gave me many chances of presentation and discussion.

My many thanks also go to Yoshihiro Seiya and Satoru Uozumi for their helps of Plug Preradiator (PPR). In addition to many comments for PPR works, Yoshi gave me useful suggestions for this analysis. Satoru kindly instructed me in CDF software usage.

I should thank my "U.S. life style adviser," Nobuaki Oshima. I will not forgot to have lunch and dinner with him (and his wife) at Jurin, Sushi House, Kurumaya, etc. with a lot of enjoyment. He also gave me many comments for my difficulties. I could not get a next postdoc position without his appropriate comments.

In terms of my job search, Wei-Ming Yao, Jason Nielsen, Beate Heinemann, Stephan Lammel, Thomas Junk, Young-Kee Kim, Fumihiko Ukegawa and Takasumi Maruyama were very kind enough to write reference letters for my postdoc applications. I believe their letters gave me great helps.

I wish to thank members of the CDF collaboration and the Tsukuba high energy physics group for their continuous help. They include Koji Takikawa, Toru Okusawa, Makoto Shimojima, Akiya Miyamoto, Yoshiaki Fujii, Kazuhiko Hara, Yuji Takeuchi, Yukihiro Kato, Hiroyuki Matsunaga, Takashi Suzuki, Hirokazu Kobayashi, Junji 
Naganoma and other colleagues. Kazuko Kumashiro, Kyoko Kunori, Barb Perington and Carol Picciolo helped me with their excellent secretary works too.

Finally, I would like to express my great appreciation to my parents. They supported me throughout my student career.

I thank the Fermilab staff and the technical staffs of the participating institutions for their vital contributions. This work was supported by the U.S. Department of Energy and National Science Foundation; the Italian Istituto Nazionale di Fisica Nucleare; the Ministry of Education, Culture, Sports, Science and Technology of Japan; the Natural Sciences and Engineering Research Council of Canada; the National Science Council of the Republic of China; the Swiss National Science Foundation; the A.P. Sloan Foundation; the Bundesministerium fuer Bildung und Forschung, Germany; the Korean Science and Engineering Foundation and the Korean Research Foundation; the Particle Physics and Astronomy Research Council and the Royal Society, UK; the Russian Foundation for Basic Research; the Comision Interministerial de Ciencia y Tecnologia, Spain; in part by the European Community's Human Potential Programme under contract HPRN-CT-20002, Probe for New Physics; and by the Research Fund of Istanbul University Project No. 1755/21122001. 


\section{Outline}

A central mystery in elementary particle physics is the mechanism of electroweak symmetry breaking, by which weak vector bosons acquire non-zero masses. The physical manifestation of electroweak symmetry breaking could be the Standard Model Higgs Boson $(H)$ or a new particle responsible for the dynamics of a new interaction. With the top mass measurement from the CDF and D $\varnothing$ collaborations in the Tevatron Run 1 experiment, a global fit of the electroweak precision data yields an estimate of the Standard Model Higgs boson mass $m_{H}=129_{-49}^{+74} \mathrm{GeV} / c^{2}$ or $m_{H}<285 \mathrm{GeV} / c^{2}$ at $95 \%$ confidence level (C.L.). This estimate is consistent with results from direct searches at Large Electron-Positron collider (LEP2) that place limits on $m_{H}>114.4 \mathrm{GeV} / c^{2}$ at $95 \%$ C.L. Higgs boson searches at the Tevatron may be sensitive to the Higgs boson production at these masses. The CDF collaboration previously performed searches for such new particles that decay into a bottom quark pair $(b \bar{b})$ using data collected during Run 1 at the Tevatron. Similar searches have been performed by the $\mathrm{D} \varnothing$ collaboration using their Run 2 data.

In this thesis a search for the Higgs boson of the Standard Model in protonantiproton $(p \bar{p})$ collisions at a center-of-mass energy of $1.96 \mathrm{TeV}$ is described. The data were collected with the Collider Detector at Fermilab (CDF) from February 2002 to August 2004. The corresponding integrated luminosity is $319 \mathrm{pb}^{-1}$. The search signature considered here is $W H$ with $W \rightarrow \ell \nu$ and $H \rightarrow b \bar{b}$, giving final states with one high- $p_{T}$ lepton, an imbalance of the total transverse energy a.k.a. missing $E_{T}\left(\mathbb{E}_{T}\right)$ and two $b$ quark jets, where $W, \ell$ and $\nu$ mean either a $W^{+}$boson or $W^{-}$boson, lepton (electron or muon) and neutrino, respectively. We focus our attention on the $W+2$ jets signature using $b$-tagging, since it contains most of the signal, while $b$-tagged $W+\geq 3$ jets events are dominated by top quark pair $(t \bar{t})$ production.

The CDF detector is an azimuthally and forward-backward symmetric apparatus designed to explore $p \bar{p}$ collisions at the Tevatron. The detector has a charged particle tracking system immersed in a 1.4 Tesla magnet field aligned coaxially with the colliding beams. A silicon microstrip detector provides tracking over the radial range from 1.5 to $28 \mathrm{~cm}$. A $3.1 \mathrm{~m}$ long open-cell drift chamber, the Central Outer Tracker (COT), covers the radial range from 40 to $137 \mathrm{~cm}$. The fiducial region of the silicon detector extends to $|\eta| \sim 2$, while the COT provides coverage for $|\eta|<$ 1. Segmented electromagnetic and hadronic sampling calorimeters surround the tracking system and measure the energy flow of interacting particles in the pseudorapidity range $|\eta|<3.6$. The electromagnetic calorimeters are instrumented with proportional and scintillating strip detectors which measure the transverse profile of the electromagnetic shower candidates at a depth corresponding to the shower maximum. A set of drift chambers located outside the central hadron calorimeters and another set behind a $60 \mathrm{~cm}$ iron shield detect energy deposition from muon candidates with $|\eta|<0.6$. Additional drift chambers and scintillation counters detect muons in the region $0.6<|\eta|<1.0$. The beam luminosity is determined using gas Cerenkov counters located in the $3.7<|\eta|<4.7$ region; the counters measure the average number of inelastic $p \bar{p}$ collisions per bunch crossing. 
The event selection begins with the requirement of a primary lepton, either an isolated electron with $E_{T}>20 \mathrm{GeV}$ or an isolated muon with $p_{T}>20 \mathrm{GeV} / c$, in the central region $(|\eta|<1.0)$. A $W$ boson sample is selected by requiring $\mathscr{E}_{T}>20 \mathrm{GeV}$. Events that contain a second, same-flavor lepton with $p_{T}>10 \mathrm{GeV} / c$ are removed as possible $Z^{0}$ boson candidates, if the reconstructed $e^{+} e^{-}$or $\mu^{+} \mu^{-}$invariant mass is between 76 and $106 \mathrm{GeV} / c^{2}$. We also reject dilepton events consistent with $t \bar{t}$ production. To further reduce the dilepton backgrounds, we reject events with an additional high- $p_{T}$ isolated track $\left(p_{T}>20 \mathrm{GeV} / c\right)$ whose charge is opposite that of the primary lepton. The remaining events are classified according to jet multiplicity. A jet is defined as a cluster of $E_{T}$-weighted calorimeter towers within a fixed radius $\Delta R=\sqrt{(\Delta \phi)^{2}+(\Delta \eta)^{2}}=0.4$, and is required to have $E_{T}>15 \mathrm{GeV}$ and $|\eta|<2.0$ after corrections for calorimeter response and multiple interactions. We veto events that have any jets with $E_{T}>8 \mathrm{GeV}$ and $|\eta|>2.0$ as well as events that have jets with $8<E_{T}<15 \mathrm{GeV}$ and $|\eta|<2.0$.

The sample of events with $W+2$ jets is expected to contain most of the signal, while the sample of events with only 1 jet is used to constrain the $W+$ heavy flavor ( $b$ quark and charm $(c)$ quark) backgrounds, and the sample of events with 3 or more jets is used to estimate the $t \bar{t}$ contributions. In order to enhance the signal purity of the $W+2$ jets sample, we require at least one jet be tagged by the secondary vertex $b$-tagging algorithm (SecVtx) as containing a $B$ hadron. This procedure depends on the precise track measurements made possible by the silicon microstrip detector. The SecVtx searches for secondary vertices formed by two or more displaced tracks. A jet is declared as "tagged," if it contains a secondary vertex with a significant transverse displacement from the primary vertex.

Background events come predominantly from the direct production of $W$ bosons in association with heavy quarks $(W+b \bar{b}, W+c \bar{c}$ and $W+c), W$ production in association with light flavor/gluon jets that are mistagged as $b$ quark jets, $t \bar{t}$ and single top production ( $W^{*} \rightarrow t b$ and $g W \rightarrow t b$, where $g$ means a gluon), and jet events without $W$ bosons. Other small backgrounds include diboson $\left(W^{+} W^{-}, W Z^{0}\right.$ and $Z^{0} Z^{0}$ ) and $Z^{0} \rightarrow \tau^{+} \tau^{-}$production.

The fraction of $W+$ jets events that contain heavy quarks is estimated using the ALPGEN Monte Carlo program and is further calibrated using the inclusive jet data. We find this fraction must be scaled by an additional factor of $1.2 \pm 0.2$ to match the observed number of tagged $W+1$ jet events. The estimated number of background events is then obtained by multiplying the heavy flavor fraction, the event tagging efficiency and the number of $W+$ jets events in the data before tagging.

To estimate the number of false tags of light flavor/gluon jets, we first parameterize the negative tag rate in an inclusive jet sample as a function of jet $E_{T}$, track multiplicity, $\eta, \phi$ and the summed $E_{T}$ of all the jets in the event. Negative tags (with vertex displacement opposite the jet direction) are a good approximation of positive mistags (vertex displacement along the jet direction), since mistags are mostly due to symmetric resolution effects. Long-lived particles and secondary interactions with the detector material contribute asymmetrically to the positive tag rate and cannot be predicted from the negative tags. By analyzing inclusive jet data, we find the rate of negative tags must be increased by a factor of $1.27 \pm 0.13$ to account for the 
asymmetries. We apply the same correction to the $W+$ jets sample to estimate the expected number of positive mistagged events.

The remaining backgrounds are estimated from a combination of Monte Carlo

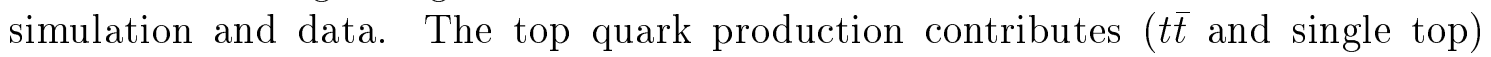
are estimated using HERWIG and PYTHIA Monte Carlo calculations normalized to the cross section measured in the same dataset $\left(\sigma_{t \bar{t}}=8.6 \pm 1.3 \mathrm{pb}\right)$ or to NLO calculations, respectively, for a top quark mass of $175 \mathrm{GeV} / c^{2}$. The other substantial background comes from events without $W$ bosons. These events are typically jet events where one of the jets has faked a high- $p_{T}$ lepton and mismeasured energies produce apparent $\mathbb{E}_{T}$. We measure this "non- $W$ " background by extrapolating the number of tagged events with an isolated lepton and low $\mathbb{E}_{T}$ into the signal region.

As a results, we observe 187 events which is in agreement with the Standard Model background expectation of $175.2 \pm 26.3$ events. The number of observed tagged events is in good agreement with the corresponding background estimations.

The acceptance for identifying $W H \rightarrow \ell \nu b \bar{b}$ events using the cuts described above is calculated as a function of $m_{H}$ from PYTHIA Monte Carlo samples of $W H \rightarrow W b \bar{b}$ events after full simulation of the CDF detector response. The total acceptance is calculated as a product of the $W$ leptonic branching fraction, the kinematic and geometric acceptance, the lepton identification efficiencies, the trigger efficiencies and $b$-tagging efficiencies. A $11 \%$ systematic uncertainty comes from uncertainties in the modeling of initial and final state radiation, parton distribution function, jet energy scale, $b$-tagging efficiency, jet energy resolution, electron and muon trigger efficiencies. The acceptance increases linearly from $1.5 \pm 0.2 \%$ to $1.7 \pm 0.2 \%$ as $m_{H}$ increases from $110 \mathrm{GeV} / c^{2}$ to $150 \mathrm{GeV} / c^{2}$.

We perform a direct search for a resonant mass peak in the reconstructed dijet invariant mass distribution. The dijet mass distributions in the at least one $b$ tagged data is in good agreement with the background expectations. In addition to the search with at least one $b$-tagging requirement, we examine a search with double $b$-tagging requirements as the cross-check. We require one of the jets to be tagged by SecVtx and the second jet to be also tagged by SecVtx. As a result, the observed number of events and dijet mass distribution are consistent with the estimated number of events and mass distribution.

Since there is no significant mass peak observed, we set an upper limit on the production cross section times branching ratio decaying into $b \bar{b}, \sigma(p \bar{p} \rightarrow W H) \times$ $\mathcal{B} r(H \rightarrow b \bar{b})$, as a function of $m_{H}$ using the number of events in the $W+2$ jets sample. A binned maximum likelihood technique is used to estimate the upper limits on the $W H$ production by constraining the number of background expectations within the uncertainties. The resultant $95 \%$ confidence level upper limits are $10.0 \mathrm{pb}$ to 2.8 $\mathrm{pb}$ using at least one $b$-tagging method and $9.7 \mathrm{pb}$ to $6.6 \mathrm{pb}$ using double $b$-tagging method for the Higgs boson mass region $110 \mathrm{GeV} / c^{2}$ to $150 \mathrm{GeV} / c^{2}$, where the Standard Model prediction is approximately one or two order of magnitude lower than the results. 



\section{Contents}

1 Introduction 1

2 The Standard Model $\quad \mathbf{5}$

2.1 Symmetry Principles and Gauge Invariance . . . . . . . . . . . . . 5

2.2 Abelian Gauge Transformations . . . . . . . . . . . . . . . 6

2.2.1 Global Gauge Transformations . . . . . . . . . . . . 6

2.2.2 Local Gauge Transformations . . . . . . . . . . . . . 6

2.3 Non-Abelian Gauge Transformations . . . . . . . . . . . . . . . 7

2.4 Electroweak Theory . . . . . . . . . . . . . . . . . . 8

2.5 The Higgs Mechanism . . . . . . . . . . . . . . . 10

2.5.1 Spontaneous Symmetry Breaking . . . . . . . . . . . 11

2.5.2 The Higgs Boson in the Standard Model . . . . . . . . . . . 12

2.6 The Higgs Boson Production and Decay at the Tevatron . . . . . . . 15

2.6.1 The Higgs Boson Production . . . . . . . . . . . . . . 15

2.6.2 The Higgs Boson Decay . . . . . . . . . . . . . 16

2.6.3 Main Search Channels at Tevatron . . . . . . . . . . 17

2.7 Review of the Previous Higgs Boson Search . . . . . . . . . . . 18

2.7.1 Direct Search at LEP . . . . . . . . . . . . . . 18

2.7 .2 Indirect Search . . . . . . . . . . . . . . . 18

2.7.3 Direct Search at the Tevatron Run 1 . . . . . . . . . 19

2.7.4 Direct Search at the Tevatron Run 2 . . . . . . . . . 19

3 Experimental Apparatus 25

3.1 The Tevatron and the Fermilab Accelerator System . . . . . . . . . 25

3.1.1 Proton Injector . . . . . . . . . . . . . 25

3.1.2 Antiproton Production and Recycling . . . . . . . . . . 27

3.1.3 The Tevatron Storage Ring . . . . . . . . . . . . . . 27

3.2 The Collider Detector at Fermilab . . . . . . . . . . . . 28

3.2.1 The CDF Coordinate System . . . . . . . . . . . 29

3.2.2 The Solenoid . . . . . . . . . . . . . 30

3.2.3 The Silicon Vertex Detector .............. 30

3.2.4 The Central Outer Tracker . . . . . . . . . . . . 31

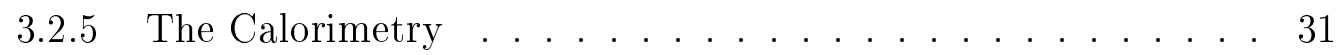

3.2 .6 The Muon System .................... 36

3.2.7 The Čerenkov Luminosity Counter . . . . . . . . . . 38 
3.2 .8 The Trigger System . . . . . . . . . . . . . . . . 40

3.2 .9 Detector Simulation . . . . . . . . . . . . . . . 41

4 Dataset and Event Selection 43

4.1 Tracking Reconstruction . . . . . . . . . . . . . . . . . 43

4.1 .1 COT Tracking . . . . . . . . . . . . . . . . 43

4.1.2 Silicon Detector Tracking. . . . . . . . . . . . . . . . . 44

4.2 Electron and Photon Identification $\ldots \ldots \ldots \ldots . \ldots . \ldots$

4.3 Muon Identification . . . . . . . . . . . . . . . . . . . 47

4.4 Jet Reconstruction . . . . . . . . . . . . . . . . . . . . . 48

4.5 Missing Transverse Energy Measurement . . . . . . . . . . . . . 49

4.6 High- $p_{T}$ Lepton Sample . . . . . . . . . . . . . . . 50

4.6 .1 Triggers . . . . . . . . . . . . . . 50

4.6 .2 Offline Selections . . . . . . . . . . . . . . 51

5 Secondary Vertex b-Tagging: The SecVtx Algorithm 53

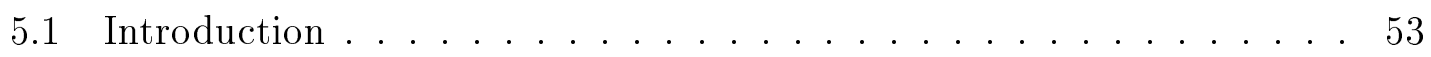

5.2 Event-by-Event Primary Vertex Finding . . . . . . . . . . . 54

5.3 The SecVtx Algorithm . . . . . . . . . . . . . . . . . 54

5.4 Track Selection . . . . . . . . . . . . . . . . 56

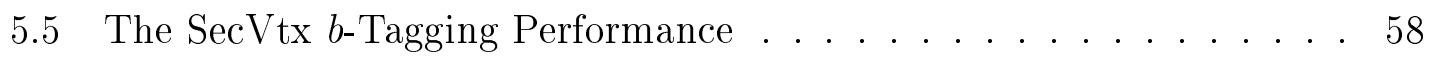

6 Background Estimation $\quad 59$

6.1 Non- $W$ Background . . . . . . . . . . . . . . . . . . . . . . . . 59

6.1.1 Pretag Non- $W$ Background . . . . . . . . . . . . 60

6.1 .2 Tagged Non- $W$ Background $\ldots \ldots \ldots$. . . . . . . . . . . 60

6.1 .3 Systematic Uncertainty . . . . . . . . . . . . . . . . 6 61

6.1.4 Summary of Non- $W$ Background $\ldots \ldots \ldots$. . . . . . 61

$6.2 W+$ Light Flavor/Gluon Jets (Mistags) $\ldots \ldots \ldots$

$6.3 W+$ Heavy Flavor Jets $(W+b \bar{b}, W+c \bar{c}$ and $W+c) \ldots \ldots 4$

6.4 Other Backgrounds ( $t \bar{t}$, single top, $W^{+} W^{-}, W Z^{0}, Z^{0} Z^{0}$ and $Z^{0} \rightarrow$

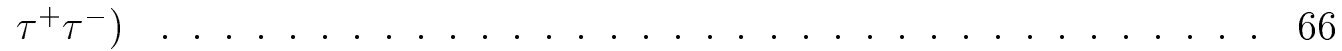

6.5 Summary of Background Estimation $\ldots \ldots \ldots 6$

7 Search for the Higgs Boson Decaying into $b \bar{b}$ and Produced in Association with $W$ Boson $\quad 69$

7.1 Event Selection Optimization _. . . . . . . . . . . . . . . 69

7.2 Dijet Mass Distribution for the Higgs Boson . . . . . . . . . . 71

7.3 Dijet Mass and $W$ Transverse Mass Shapes for Non- $W$ Background . 71

7.4 Validation of Kinematic Distributions . . . . . . . . . . . 78

7.5 Dijet Mass Shape . . . . . . . . . . . . . . . 78

7.6 Dijet Mass Distribution . . . . . . . . . . . . . . . 79 
8 Upper Limit on the Cross Section 89

8.1 Procedure for Setting Limit . . . . . . . . . . . . . 89

8.2 Signal Acceptance . . . . . . . . . . . . . . . . 90

8.3 A 95\% Confidence Level Limit on the Higgs Boson Production . . . . 96

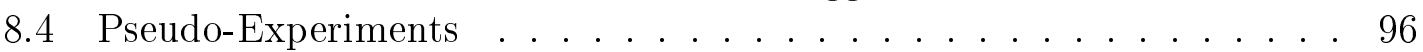

8.5 Double $b$-Tagging as an Cross Check . . . . . . . . . . . . . 99

8.6 Other Direct Standard Model Higgs Boson Searches at Tevatron . . . 99

8.7 Future Prospect . . . . . . . . . . . . . . 105

$\begin{array}{lll}9 & \text { Conclusion } & 107\end{array}$

$\begin{array}{ll}\text { A Non- } W \text { Background } & 109\end{array}$

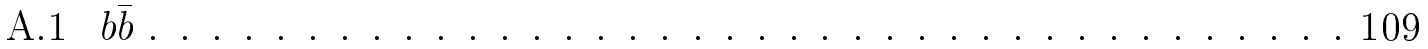

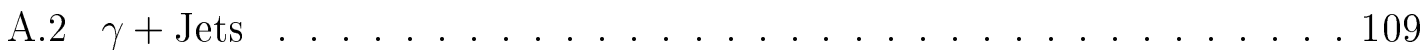

A.3 $W(\rightarrow \tau \nu)+$ Jets and $Z^{0}\left(\rightarrow \mu^{+} \mu^{-}\right)+$Jets . . . . . . . . . . 112

A.4 Dijet Mass and $W$ Transverse Mass Distributions . . . . . . . . . . 115

A.5 A Cross Check with Additional Cut on Non- $W \ldots . . . . . .116$

B Kolmogorov-Smirnov Test $\quad 119$ 



\section{List of Figures}

1.1 The Feynman diagrams of $p \bar{p} \rightarrow W H \rightarrow \ell \nu b \bar{b} . \ldots . \ldots$

2.1 Potential $V(\Phi)=(1 / 2) \mu^{2} \Phi^{2}+(1 / 4) \lambda \Phi^{4}\left(\mu^{2}<0\right.$ and $\left.\lambda>0\right)$. . . . 11

2.2 The production cross section of the Higgs boson as a function of the mass at the Tevatron. . . . . . . . . . . . . 15

2.3 The Feynman diagram of gluon-gluon fusion process. . . . . . . . . 15

2.4 The branching ratio of the Higgs boson as a function of the mass. . . 17

2.5 The Feynman diagrams of the Higgs boson productions which are promising channels at the Tevatron. (a) $p \bar{p} \rightarrow W H \rightarrow \ell \nu b \bar{b}$, (b)p $\bar{p} \rightarrow$ $Z^{0} H \rightarrow \nu \bar{\nu} b \bar{b},(\mathrm{c}) p \bar{p} \rightarrow Z^{0} H \rightarrow \ell^{+} \ell^{-} b \bar{b},(\mathrm{e}) g \bar{g} \rightarrow H \rightarrow W^{+} W^{-} \rightarrow$ $\ell^{+} \nu \ell^{-} \bar{\nu},(\mathrm{d}) p \bar{p} \rightarrow W H \rightarrow W W^{+} W^{-} \rightarrow \ell^{ \pm} \nu \ell^{ \pm} \nu q \bar{q} . \ldots . . . . .20$

2.6 Distributions of the reconstructed Higgs boson mass, $m_{H}^{\text {rec }}$, obtained from two selections with different expected signal purities. (a)Loose selections, (b)Tight selections. . . . . . . . . . . . 21

2.7 The Standard Model relationship for $m_{W}$ and $m_{t}$ as a function of the Higgs mass. The arrow labeled $\Delta \alpha$ shows the variation of this relation if $\alpha\left(m_{Z}^{2}\right)$ is changed by one standard deviation. This variation gives an additional uncertainty to the Standard Model band shown in the

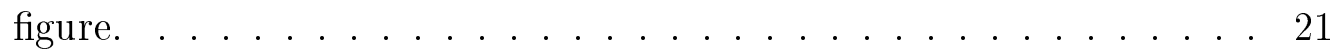

2.8 The $\Delta \chi^{2}$ distribution as a function of $m_{H}$ from a global fit (with only $m_{H}$ as a free parameter) using the precision electroweak measurements from LEP, SLD, CDF, DØ and NuTeV. . . . . . . . . 22

2.9 The $95 \%$ C.L. upper limits for the Standard Model Higgs boson production cross section from CDF Run 1 data. . . . . . . . . . 22

2.10 The $95 \%$ C.L. upper limits for the Standard Model Higgs boson production cross section from D $\varnothing$ Run 2 data. . . . . . . . . . 23

3.1 The chain of accelerators at Fermilab. . . . . . . . . 26

3.2 Diagram of one half of the CDF Run 2 detector. . . . . . . . . . 28

3.3 Tracking system of the CDF detector. . . . . . . . . . . . 29

3.4 CDF silicon tracking detector in $r \times \phi$ plane. . . . . . . . . 30

3.5 CDF silicon tracking detector in $r \times z$ plane. . . . . . . . . . . 31

3.6 Cell layout for super layer 2. . . . . . . . . . . . . . . . . . . . . . . . . . . 32

$3.71 / 6$ th view of COT east end plate. . . . . . . . . . . . 33

3.8 A isometric view of the central calorimeter wedge. . . . . . . . . 34

3.9 Cross section of upper part of end plug calorimeter. . . . . . . . . 35 
3.10 Segmentation of the plug calorimeter. . . . . . . . . . . 36

$3.11 \eta$ and $\phi$ coverage of the CDF muon system. . . . . . . . . . 37

3.12 Number of nuclear absorption lengths in front of the muon systems as a function of pseudo-rapidity. . . . . . . . . . . . 38

3.13 Section of a CMU module in the $x \times y$ plane. . . . . . . . . 39

3.14 The CDF Čerenkov Luminosity Counter. . . . . . . . . . . . . . 40

3.15 Time distribution in the East and West modules. Actual $p \bar{p}$ collisions deposit a coincident signal in the two modules. . . . . . . . . . . 41

3.16 The CDF Trigger system. (Left)schematic view of DAQ and trigger system, (Right)block diagram of the Level 1 and Level 2 triggers. . . 42

5.1 Schematic view of a positive (left) and negative (right) SecVtx tag. . 55

5.2 Vertex display of a $W+2$ jets candidate event with two SecVtx positive tags (run 166063, event 279746). Only tracks within a jet cone and passing the selection are shown. Prompt tracks, a muon track and tracks of the final secondary vertex fit are shown in solid black, solid blue and solid red. The $\mathbb{E}_{T}$ direction is also shown in a green arrow. . 56

5.3 The $b$-tagging efficiency to tag $b$ quark jets in $t \bar{t}$ MC samples which have been matched to $b$ quarks, using both the "tight" and "loose" tunes of the SecVtx tagger as functions of jet $E_{T}$ (left) and $\eta$ (right). The bands represent the systematic error on the $\varepsilon_{\text {data }} / \varepsilon_{\mathrm{MC}}$ scale factors. 58

5.4 The mistag rate for jets resulting from light quark/gluon fragmentation as functions of jet $E_{T}$ (left) and $\eta$ (right). These have been measured from inclusive jet data. . . . . . . . . . . 58

6.1 Comparison of observed and predicted negative SecVtx tags as a function of jet multiplicity in the lepton + jets data sample. (a)electron channel, (b)muon channel. . . . . . . . . . . . . . . . . 64

6.2 The number of observed $W+$ positive tagged events and the background summary as a function of jet multiplicity. The integrated luminosities are $318.5 \mathrm{pb}^{-1}$ for CEM and CMUP, and $305.2 \mathrm{pb}^{-1}$ for CMX .......................

7.1 The significance as a function of 1 st and 2 nd leading jet $E_{T}$ selection criteria. The significance is not sensitive to the 1st leading jet $E_{T}$. (a) $m_{H}=110 \mathrm{GeV} / c^{2}$, (b) $m_{H}=115 \mathrm{GeV} / c^{2}$, (c) $m_{H}=120 \mathrm{GeV} / c^{2}$, (d) $m_{H}=130 \mathrm{GeV} / c^{2}$, (e) $m_{H}=140 \mathrm{GeV} / c^{2}$, (f) $m_{H}=150 \mathrm{GeV} / c^{2}$. . 70

7.2 The jet multiplicity distribution after event selections using $W H \mathrm{MC}$ samples. (a) $m_{H}=110 \mathrm{GeV} / c^{2}$, (b) $m_{H}=115 \mathrm{GeV} / c^{2}$, (c) $m_{H}=120$ $\mathrm{GeV} / c^{2}$, (d) $m_{H}=130 \mathrm{GeV} / c^{2}$, (e) $m_{H}=140 \mathrm{GeV} / c^{2}$, (f) $m_{H}=150$ $\mathrm{GeV} / c^{2}$. The $W+2$ jets signature contains most of the signal events.

7.3 The reconstructed dijet mass distributions for the Higgs boson using $W H$ MC samples in $W+2$ jets bin. (a) $m_{H}=110 \mathrm{GeV} / c^{2}$, (b) $m_{H}=$ $115 \mathrm{GeV} / c^{2}$, (c) $m_{H}=120 \mathrm{GeV} / c^{2}$, (d) $m_{H}=130 \mathrm{GeV} / c^{2}$, (e) $m_{H}=$ $140 \mathrm{GeV} / c^{2}$, (f) $m_{H}=150 \mathrm{GeV} / c^{2} \ldots \ldots \ldots \ldots \ldots$ 
7.4 The Gaussian central value and width, the mass deviation and the mass resolution. (a)Gaussian central value, (b)Gaussian width, (c)mass deviation, $(\mathrm{d})$ mass resolution. . . . . . . . . . . . 74

7.5 The lepton $E_{T}$ distributions for the $W H$ tagged sample, where $m_{H}=$ $115 \mathrm{GeV} / c^{2}$ is assumed. (a)electron channel, (b)muon channel, (c)electron + muon channel. . . . . . . . . . . . . . . . 74

7.6 The $\mathbb{E}_{T}$ distributions for the $W H$ tagged sample, where $m_{H}=115$ $\mathrm{GeV} / c^{2}$ is assumed. (a)electron channel, (b)muon channel, (c)electron + muon channel. . . . . . . . . . . . . . . . 75

7.7 The $W$ transverse mass distributions for the $W H$ tagged sample, where $m_{H}=115 \mathrm{GeV} / c^{2}$ is assumed. (a)electron channel, (b)muon channel, (c)electron + muon channel. . . . . . . . . . . . . . 75

7.8 The 1st leading jet $E_{T}$ distributions for the $W H$ tagged sample, where $m_{H}=115 \mathrm{GeV} / c^{2}$ is assumed. (a)electron channel, (b)muon channel, (c)electron + muon channel. . . . . . . . . . . . . . 76

7.9 The 2nd leading jet $E_{T}$ distributions for the $W H$ tagged sample, where $m_{H}=115 \mathrm{GeV} / c^{2}$ is assumed. (a)electron channel, (b)muon channel, (c)electron + muon channel. . . . . . . . . . 76

7.10 The comparisons of dijet and $W$ transverse mass in the sideband Regions C, A', B' and in the conversion sample, where the upper isolation cut is lowered from 0.2 to 0.1 for Region C. (Left)dijet mass distribution, (Right) $W$ transverse mass distribution. . . . . . . . . 77

7.11 The comparisons of pretag dijet mass prediction with data events. The plot on the left uses a non- $W$ dijet mass shape derived in Region B', and the plot on the right uses a non- $W$ dijet mass shape derived in Region C, where the upper isolation cut is lowered from 0.2 to 0.1 for Region C. . . . . . . . . . . . . . . . . . 77

7.12 The lepton $E_{T}$ distributions for $W+2$ jets pretag sample. (a)electron channel, (b)muon channel, (c)electron + muon channel. . . . . . . . 78

7.13 The $\mathbb{E}_{T}$ distributions for $W+2$ jets pretag sample. (a)electron channel, (b)muon channel, (c)electron + muon channel. . . . . . . . . 79

7.14 The $W$ transverse mass distributions for $W+2$ jets pretag sample. (a)electron channel, (b)muon channel, (c)electron + muon channel. . 80

7.15 The 1st leading jet $E_{T}$ distributions for $W+2$ jets pretag sample. (a)electron channel, (b)muon channel, (c)electron + muon channel. $\quad 80$

7.16 The 2nd leading jet $E_{T}$ distributions for $W+2$ jets pretag sample. (a)electron channel, (b)muon channel, (c)electron + muon channel. . 81

7.17 The lepton $E_{T}$ distributions for $W+2$ jets tagged sample. (a)electron channel, (b)muon channel, (c)electron + muon channel. . . . . . . . 81

7.18 The $\mathbb{E}_{T}$ distributions for $W+2$ jets tagged sample. (a)electron channel, (b)muon channel, (c)electron + muon channel. . . . . . . . . . 82

7.19 The $W$ transverse mass distributions for $W+2$ jets tagged sample. (a)electron channel, (b)muon channel, (c)electron + muon channel. $\quad 82$

7.20 The 1st leading jet $E_{T}$ distributions for $W+2$ jets tagged sample. (a)electron channel, (b)muon channel, (c)electron + muon channel. . 83 
7.21 The 2nd leading jet $E_{T}$ distributions for $W+2$ jets tagged sample. (a)electron channel, (b)muon channel, (c)electron + muon channel. . 83

7.22 The lepton $E_{T}$ distributions for $W+1$ jet tagged sample. (a)electron channel, (b)muon channel, (c)electron + muon channel. . . . . . . . . 84

7.23 The $\not_{T}$ distributions for $W+1$ jet tagged sample. (a)electron channel, (b)muon channel, (c)electron + muon channel. . . . . . . . . . . 84

7.24 The $W$ transverse mass distributions for $W+1$ jet tagged sample. (a)electron channel, (b)muon channel, (c)electron + muon channel. . 85

7.25 The 1st leading jet $E_{T}$ distributions for $W+1$ jet tagged sample. (a)electron channel, (b)muon channel, (c)electron + muon channel. . 85

7.26 The invariant mass of the two leading jets in the $b$-tagged $W+\geq 3$ jets sample from data and expected backgrounds. . . . . . . . . . 86

7.27 The dijet mass distribution in the data along with background samples. The Higgs boson mass distribution is scaled up by a factor of 10, where the Higgs boson mass is assumed to be $115 \mathrm{GeV} / c^{2}$. . . . 87

7.28 The ratio of the numbers of events between data and background expectation as a function of the dijet mass. (Top)pretag sample, (Bottom)tagged sample. . . . . . . . . . . . 88

8.1 The logarithm of a likelihood function (top) compared to the likelihood function itself (bottom), illustrating the two different methods which can be used to derive the $95 \%$ C.L. on the parameter $\alpha$. . . . . 91

8.2 The event selection efficiency for $m_{H}=115 \mathrm{GeV} / c^{2}$ using the $W H \rightarrow$ $\ell^{\prime} \nu b \bar{b}\left(\ell^{\prime}=e, \mu, \tau\right) \mathrm{MC}$ sample in the $W+2$ jets bin. The selection number is described in Table 8.1. . . . . . . . . . . . . . . . 91

8.3 The extra jet selection efficiency for data and MC. . . . . . . . . . . 94

8.4 The overall $W H \rightarrow W b \bar{b}$ acceptance with overall systematic uncertainty $(11 \%)$ as a function of $m_{H}$. . . . . . . . . . . . 95

8.5 The $-\ln \mathcal{L}$ distributions as a function of the cross section for the dijet mass fit. . . . . . . . . . . . . . . . . . 97

8.6 The likelihood distributions as a function of the cross section for the dijet mass fit. . . . . . . . . . . . . . . . 97

8.7 The $95 \%$ C.L. upper limits (red circles) on the $W H$ cross section as a function of the Higgs boson mass. Also shown are the theoretical cross section (black triangles) for Standard Model Higgs boson production in association with a $W$ boson and the expected limit results from pseudo-experiments (blue region). . . . . . . . . . . 98

8.8 Comparison of observed limits with the expected limits from pseudoexperiments. The red line shows the measured upper limits. (a) $m_{H}=$ $110 \mathrm{GeV} / c^{2}$, (b) $m_{H}=115 \mathrm{GeV} / c^{2}$, (c) $m_{H}=120 \mathrm{GeV} / c^{2}$, (d) $m_{H}=$ $130 \mathrm{GeV} / c^{2}$, (e) $m_{H}=140 \mathrm{GeV} / c^{2}$, (f) $m_{H}=150 \mathrm{GeV} / c^{2}$. The lines show the measured upper limits. In figure, the "prob." means probability to measure the upper limit from data. . . . . . . . . . . . . . 100 
8.9 The observed and estimated number of events for (Left)at least one $b$-tagging method, (Right)double $b$-tagging method. The left plot is the same as Figure 6.2. . . . . . . . . . . . . . . . 101

8.10 The dijet mass distributions for (Left)at least one $b$-tagging method, (Right)double $b$-tagging method. The left plot is the same as Figure 7.27. . . . . . . . . . . . . . . . 102

8.11 Significance as a function of Higgs mass for the selection with at least one $b$-tagging or with double $b$-tagging. . . . . . . . . . . . . . 102

8.12 The total $W^{ \pm} H \rightarrow W b \bar{b}$ acceptance as a function of the Higgs boson mass for (Left)at least one $b$-tagging method, (Right)double $b$-tagging method. The left plot is the same as Figure 8.4. . . . . . . . . 103

8.13 The $95 \%$ C.L. upper limits (red circles) on the $W H$ cross section as a function of the Higgs boson mass for double $b$-tagged events. Also shown are the theoretical cross section (black triangles) for Standard Model Higgs boson production in association with a $W$ boson, and the expected limit results from pseudo-experiments (blue region). . . 104

8.14 The current Higgs boson search results at Tevatron. . . . . . . . . . 105

8.15 The Higgs boson search sensitivity at Tevatron. . . . . . . . . . . 106

A.1 The $b$-tagging rates in $b \bar{b}$ decays as functions of $(a) \mathbb{E}_{T}$ and (b)lepton isolation. . . . . . . . . . . . . . . . . 110

A.2 The dijet and $W$ transverse mass distributions from $b \bar{b}$ and fake $\left(\pi^{0}\right.$ decays) samples after the standard selection. (Top) $W$ transverse mass, (Bottom)dijet mass, (Left)electron channel, (Right)muon channel. . . . . . . . . . . . . . . . . . 111

A.3 Some kinematic distributions for pretag $\gamma+$ jets events. (Upper left)Lepton $E_{T}$, (Upper right) $\mathbb{E}_{T}$, (Middle left) $W$ transverse mass, (Middle right)1st leading jet $E_{T}$, (Lower left)2nd leading jet $E_{T}$, (Lower right)dijet mass. . . . . . . . . . . . . . . . 113

A.4 The distributions of dijet and $W$ transverse mass from $W \rightarrow \tau \nu$ and $Z^{0} \rightarrow \mu^{+} \mu^{-}$. (Top) $W \rightarrow \tau \nu$ sample, (Bottom) $Z^{0} \rightarrow \mu^{+} \mu^{-}$sample, (Left) $W$ transverse mass, (Right)dijet mass. . . . . . . . . . . . 114

A.5 The comparison of $W$ transverse mass in data and expected shape (see text). (a)electron channel, (b)muon channel, (c)electron + muon channel. . . . . . . . . . . . . . . . . 115

A.6 The comparison of dijet mass in data and expected shape (see text). (a)electron channel, (b)muon channel, (c)electron + muon channel. . . 116

A.7 The comparisons of dijet mass distribution from data and expected background with (left) and without (right) the $\Delta \phi$ cut. The left plot is the same as Figure 7.27. . . . . . . . . . . . . . . 117 



\section{List of Tables}

1.1 Three generations of leptons and quarks. . . . . . . . . . . 2

2.1 Multiplet and quantum number assignments for the fermions in the Standard Model. The prime indicates that the weak eigenstates of the quarks are not their mass eigenstates. The quark mixing is described by the Cabibbo-Kobayashi-Maskawa matrix. The indices $L$ (R) denote left (right)-handed fermions. . . . . . . . . . . 9

2.2 Quantum number assignments of the bosons in the Standard Model. . 14

3.1 Some properties of the CDF calorimeters. The last row shows the single particle energy resolution. . . . . . . . . . 36

4.1 CEM electron selection criteria. . . . . . . . . . . . 47

4.2 CMUP and CMX muon selection criteria. . . . . . . . . . . 48

5.1 Bins used to define the track classes. For tracks with 3 hits, the hit pattern (i.e. what layers the hits are on) is also considered. . . . . . 57

6.1 The ratio of extrapolated and observed non- $W$ backgrounds in Regions F and B' for pretag and $b$-tagged event samples. . . . . . . . . 62

6.2 The estimated number of $b$-tagged non- $W$ events (Region $\mathrm{B}$ rate

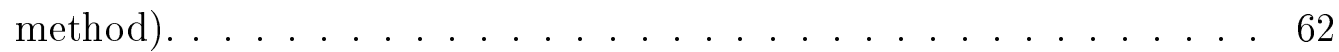

6.3 The estimated number of $b$-tagged non- $W$ events (tagged method). $\quad 62$

6.4 The estimated number of $b$-tagged non- $W$ events (average). . . . . . . 62

6.5 The estimated number of mistag background contribution. . . . . . . 63

6.6 Ratio of $W+$ heavy flavor production to total $W+$ jets production for different jet multiplicities. The SecVtx event tagging efficiencies include the $b$-tagging scale factor of $0.909 \pm 0.060$. The samples of $W+b \bar{b}$ and $W+c \bar{c}$ are further divided into two classes based on the number of heavy flavor jets $\left(E_{T}>15 \mathrm{GeV}\right.$ and $\left.|\eta|<2.4\right)$ inside the detector. . . . . . . . . . . . . . 65

6.7 The estimated $W+$ heavy flavor background contributions. . . . . . . 65

6.8 The theoretical and measured cross sections used to estimate the MC derived background contributions. . . . . . . . . . 66

6.9 The number of observed $W+$ positive tagged events and the background summary for an integrated luminosity of $318.5 \mathrm{pb}^{-1}$ for CEM and CMUP, and $305.2 \mathrm{pb}^{-1}$ for CMX. . . . . . . . . . 67 
8.1 The definition of event selection numbers. . . . . . . . . . . 92

8.2 Systematic uncertainties on the signal acceptance. . . . . . . . . . 94

8.3 The fit values with errors and the $95 \%$ C.L. upper limits. . . . . . . 99

8.4 The number of observed double positive tagged events and the background summary for an integrated luminosity of $318.5 \mathrm{pb}^{-1}$ for CEM and CMUP and $305.2 \mathrm{pb}^{-1}$ for CMX. . . . . . . . . . . 101

8.5 The numbers of signal and background in $1.5 \sigma$ mass window for $m_{H}=$ $115 \mathrm{GeV} / c^{2} \ldots \ldots \ldots \ldots \ldots \ldots 10 \ldots \ldots \ldots$

8.6 The signal acceptance for $m_{H}=115 \mathrm{GeV} / c^{2} \ldots \ldots \ldots \ldots \ldots$

9.1 The $95 \%$ C.L. upper limits for at least one $b$-tagging and double $b$ tagging methods. . . . . . . . . . . . . 107

A.1 The ratio of predicted and observed non- $W$ background in "signal" region for $b \bar{b}$ and "fake" (electrons from $\pi^{0}$ decay) samples. . . . . . 112

A.2 The ratio of the number of events between Region D (C) and Region B (A) for $\gamma+$ jets sample. . . . . . . . . . . . . . . . . . . . 112

A.3 The comparison of the backgrounds and observed number of tags with

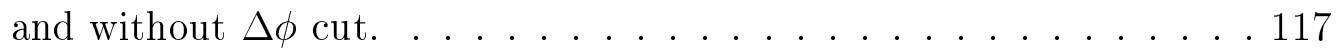




\section{Chapter 1}

\section{Introduction}

Elementary particle physics deals with questions first recorded by the philosophers in ancient Greece:

- What is the basic nature of the material world around us?

- What are the simplest, the most elementary kinds of matter?

- What are the basic forces that operate in our material world?

Although these are very old questions, it was not until the electron was discovered by J.J. Thomson about a century ago (1987) that scientists began to make appreciable progress. Currently the theoretical framework that describes the fundamental constituents of matter, which we call the elementary particles, and the basic forces that govern their behavior is a relativistic quantum field theory called the Standard Model [1]. The predictions made by the theory are in excellent agreement with experimental measurements. According to the Standard Model, there are three types of fundamental particles that interact via the four basic forces. The Standard Model successfully describes three ${ }^{1}$ of the four forces of nature: the electromagnetic, the weak and the strong. The fundamental particles are the leptons, quarks and the force carrying particles (gauge bosons). All matters are constructed from leptons and quarks, which interact via the exchange of gauge bosons.

Table 1.1 shows the leptons and quarks. The leptons and quarks are both spin 1/2 fermions. There are six types (or flavors) of leptons. Three of them are the electron $\left(e^{-}\right)$, muon $\left(\mu^{-}\right)$and tau $\left(\tau^{-}\right)$. These leptons carry electric charge of -1 and vary in mass. The other three leptons are the electron neutrino $\left(\nu_{e}\right)$, muon neutrino $\left(\nu_{\mu}\right)$ and tau neutrino $\left(\nu_{\tau}\right)$. These leptons are electrically neutral and have very small masses. The six leptons are paired into three generations. Each generation consists of a charged lepton and a neutrino. There are six distinct flavors of quarks. They are the up $(u)$, down $(d)$, charm $(c)$, strange $(s)$, top $(t)$ and bottom $(b)$. They differ in mass and carry electric charge equal to a precise fraction of an electron's charge: the $d, s$ and $b$ quarks have a charge of $-1 / 3$, and the $u, c$ and $t$ quarks have a charge of $+2 / 3$. Besides the electric charge, each quark has an additional degree

\footnotetext{
${ }^{1}$ The forth basic force, gravity, is not incorporated into the Standard Model.
} 


$$
\begin{aligned}
& \left(\begin{array}{c}
\nu_{e} \\
e^{-}
\end{array}\right)\left(\begin{array}{c}
\nu_{\mu} \\
\mu^{-}
\end{array}\right)\left(\begin{array}{c}
\nu_{\tau} \\
\tau^{-}
\end{array}\right) \\
& \left(\begin{array}{l}
u \\
d
\end{array}\right) \quad\left(\begin{array}{l}
c \\
s
\end{array}\right) \quad\left(\begin{array}{l}
t \\
b
\end{array}\right)
\end{aligned}
$$

Table 1.1: Three generations of leptons and quarks.

of freedom called color. It is labeled as Red (R), Green (G) or Blue (B), and plays the same role in strong interactions as the electric charge does in electromagnetic interactions.

The force between particles are mediated by the gauge bosons which are the remaining group of fundamental particles. They all have spin 1 . The photon $(\gamma)$ mediates the electromagnetic force, and the $W$ and $Z^{0}$ bosons $^{2}$ mediate the weak force. The strong force between quarks is transmitted by eight massless bosons called gluons $(g)$. The gluon also carries color which is a combination of a color and an anticolor.

The Standard Model has been the focus of intense scrutiny over the past three decades in which the theory has been tested to very high precision. The observations of $t$ quark [2] and $\nu_{\tau}$ [3] in experiments are the most recently discovered Standard Model particles. According to the Standard Model, these particles were guaranteed to be present, but they eluded the discoveries for many years. The observations of $t$ quark and $\nu_{\tau}$ were several in a series of convincing arguments for the validity of the Standard Model.

Despite the phenomenal success of the predictive powers of the Standard Model, there is a portion of the framework for which no evidence has been discovered. In the Standard Model, the mechanism by which the fundamental particles acquire the mass, which called the Higgs mechanism [4], has thus far eluded the experimental verification. It is the charge of this and subsequent generations of experiments to elucidate this portion of the theoretical framework. A primary current experimental goal is a search for the Higgs boson, a physically observable particle that is an additional consequence of the Higgs mechanism upon which the tenets of the Standard Model rely. Unfortunately the Standard Model does not predict the mass of the Higgs boson $\left(m_{H}\right)$; lacking a clear prediction from theory, previous direct and indirect searches considered a wide range of possible $m_{H}$. The results of these analysis indicate that a low mass Higgs boson is favored, and the mass lies within the range $114.4 \mathrm{GeV} / c^{2}<m_{H}<285 \mathrm{GeV} / c^{2}$ at $95 \%$ confidence level [5, 6].

This document describes a search for the Higgs boson in the remnants of protonantiproton collisions manufactured by the Tevatron accelerator complex at Fermi National Accelerator Laboratory. This search uses $319 \mathrm{pb}^{-1}$ data which were collected by Collider Detector at Fermilab (CDF) experiment. Figure 1.1 shows the Feynman diagrams of $p \bar{p} \rightarrow W H \rightarrow \ell \nu b \bar{b}$, where $p, W, H, \ell, \nu$ and $b$ denote a proton, $W$ boson, Higgs boson, lepton (electron or muon), neutrino and $b$ quark,

\footnotetext{
${ }^{2} W$ boson refers to both the $W^{+}$boson and its antiparticle, $W^{-}$boson.
} 
respectively ${ }^{3}$. We search for the Higgs boson decaying into $b \bar{b}$ and produced in association with a leptonically decaying $W$ boson.

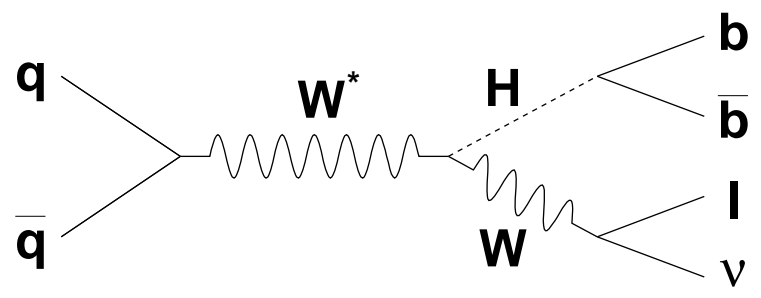

Figure 1.1: The Feynman diagrams of $p \bar{p} \rightarrow W H \rightarrow \ell \nu b \bar{b}$.

The contents of this document are organized as follows. Chapter 2 contains a description of the theoretical aspects behind the Higgs mechanism in the Standard Model, as well as present search limits. Chapter 3 describes the Tevatron accelerator complex and the CDF detector. Chapter 4 describes dataset and event selections. Chapter 5 goes into the detail of the secondary vertex $b$-tagging algorithm. The background estimations is found in Chapter 6. Chapter 7, Chapter 8 and Chapter 9 summarize the search results and conclusion.

\footnotetext{
${ }^{3}$ Although we treat the inclusive process, $p \bar{p} \rightarrow W H X \rightarrow \ell \nu b \bar{b} X$, where $X$ shows particles except $\ell \nu b \bar{b}$ in $p \bar{p}$ collisions, we omit $X$ in this thesis.
} 



\section{Chapter 2}

\section{The Standard Model}

The $\mathrm{SU}(3)_{C} \times \mathrm{SU}(2)_{L} \times \mathrm{U}(1)_{Y}$ description of fundamental particle interactions, commonly known as the Standard Model, is one of the most successful and most precisely tested theories to date. The Standard Model is based on the underlying principle of symmetry which, in physical terms, translates to the unification of forces which are seemingly different in nature. The Standard Model requires the Higgs sector to unify the observed weak and electromagnetic forces. The presence of the Higgs field allows other particles to acquire a mass term without breaking the gauge invariance of the Lagrangian.

\subsection{Symmetry Principles and Gauge Invariance}

The principle of symmetry and its application to modern particle physics theory is both powerful and far-reaching. The requirement that the Standard Model Lagrangian should conserve its properties under symmetry transformations is at the heart of all aspects of the Standard Model and many proposed extensions. This is what makes the electroweak sector of the Standard Model so attractive; starting from a very basic principle one can build the Lagrangian of the Standard Model. This is also why there is such a focus on finding the Higgs boson (or a Higgs-like particle). Without the Higgs boson the Standard Model would be incomplete as it would not be able to explain the difference in mass between the photon and the vector bosons ( $W$ and $Z^{0}$ bosons) or indeed the presence of massive particles.

To understand the electroweak sector of the Standard Model one needs to understand the basic principles on which it is based and how the Standard Model stems naturally from them. The Lagrangian for a free Dirac field under $\mathrm{SU}(2)$ and $\mathrm{U}(1)$ gauge transformations is studied in order to see how imposing the invariance of the Lagrangian under gauge transformations affects particle content and particle interactions. 


\subsection{Abelian Gauge Transformations}

\subsubsection{Global Gauge Transformations}

The Lagrangian $\mathcal{L}_{\text {Dirac }}$ for a free Dirac field $\psi(x)$ is given in the following Equation:

$$
\mathcal{L}_{\text {Dirac }}=\bar{\psi}(x)\left(i \gamma^{\mu} \partial_{\mu}-m\right) \psi(x),
$$

where $\bar{\psi}(x)$ is the conjugate field defined as $\psi^{\dagger}(x) \gamma^{0}$. The $\gamma^{\mu}$ are the $4 \times 4$ gamma matrices. Equation 2.1 is manifestly invariant under the following global gauge transformation:

$$
\psi(x) \rightarrow \psi^{\prime}(x)=e^{i q \alpha} \psi(x),
$$

where the conjugate field transforms in an equivalent way but with the opposite sign in the exponential. The $\alpha$ is a real and continuous parameter, and the $q$ is the charge of the field. The transformation in Equation 2.2 is that for an Abelian U(1) group and is global because the transformation is the same for all space-time points. The invariance of the Lagrangian under global and continuous transformations can be directly linked with a conserved current via Noether's theorem. In this example the conserved current is that of electric charge.

\subsubsection{Local Gauge Transformations}

A more interesting case is that in which the transformation is not global but has a space-time dependency i.e. the $\alpha$ in Equation 2.2 is replaced by $\alpha(x)$. Under the new transformation the Dirac Lagrangian is no longer invariant as the partial derivative in Equation 2.1 will now contribute an non-zero term from its action on the exponential. In order to impose local gauge invariance the extra $q \bar{\psi}(x) \psi(x) \gamma^{\mu} \partial_{\mu} \alpha(x)$ term from the action of the partial derivative has to be compensated for. A real gauge field $A_{\mu}(x)$ is introduced with the following gauge transformation property:

$$
A_{\mu}(x) \rightarrow A_{\mu}^{\prime}(x)=A_{\mu}(x)-\frac{1}{g} \partial_{\mu} \alpha(x)
$$

where $g$ is a real number. The Lagrangian is modified to that in Equation 2.4:

$$
\begin{aligned}
\mathcal{L} & =\bar{\psi}(x)\left(i \gamma^{\mu} \partial_{\mu}-g q \gamma^{\mu} A_{\mu}(x)-m\right) \psi(x) \\
& =i \bar{\psi}(x) \gamma^{\mu}\left(\partial_{\mu}+i g q A_{\mu}(x)\right) \psi(x)-m \bar{\psi}(x) \psi(x)
\end{aligned}
$$

where the mass term is manifestly invariant as before. The gauge transformation of $A_{\mu}(x)$ gives rise to a term $-q \bar{\psi}(x) \psi(x) \gamma^{\mu} \partial_{\mu} \alpha(x)$ which cancels the extra term from the action of the partial derivative and restores gauge invariance. The second term in Equation 2.4 describes the interaction between the Dirac and the gauge fields with a strength given by a coupling constant $(g)$ and the field charge $(q)$. In quantum electrodynamics (QED) this is the interaction between the electron and the photon field where the coupling is simply $e$, the magnitude of the electron charge.

Equation 2.4 can be rewritten in terms of a covariant derivative $D_{\mu}$. The covariant derivative has the property given in Equation 2.5, i.e. when replacing the 
partial derivative by the covariant derivative the gauge invariance of the Lagrangian follows as in the global case:

$$
D_{\mu} \psi(x) \rightarrow D_{\mu}^{\prime} \psi^{\prime}(x)=e^{i q \alpha(x)} D_{\mu} \psi(x)
$$

For QED the covariant derivative is

$$
D_{\mu} \equiv \partial_{\mu}+i e q A_{\mu}(x)
$$

In order for $A_{\mu}(x)$ to represent the photon field the Lagrangian needs to include a kinetic energy term and, in the most general case, a mass term. As the Lagrangian is now invariant under local gauge transformations, these additional terms also have to be locally gauge invariant. A kinetic term can be constructed from the antisymmetric field strength tensor $F_{\mu \nu}$ defined as

$$
F_{\mu \nu}=\partial_{\mu} A_{\nu}(x)-\partial_{\nu} A_{\mu}(x)
$$

This is invariant under the gauge transformation in Equation 2.3. The kinetic term appears as

$$
\mathcal{L}_{\gamma}^{\mathrm{K} . \mathrm{E} .}=-\frac{1}{4} F_{\mu \nu} F^{\mu \nu}
$$

A mass term for the field $A_{\mu}(x)$ would be of the form

$$
-\frac{1}{2} m_{\gamma}^{2} A_{\mu}(x) A^{\mu}(x)
$$

This is clearly not gauge invariant unless $m_{\gamma}=0$, which is perfectly consistent with observations. Therefore the Lagrangian invariant under U(1) local gauge transformation is given in Equation 2.10:

$$
\mathcal{L}=\bar{\psi}(x)\left(i \gamma^{\mu} D_{\mu}-m\right) \psi(x)-\frac{1}{4} F_{\mu \nu} F^{\mu \nu}
$$

\subsection{Non-Abelian Gauge Transformations}

The gauge transformation in Equation 2.2 can be extended trivially to non-Abelian local gauge transformations. It will be seen that local non-Abelian gauge transformations can be directly linked to the presence of the vector gauge bosons. The $\mathrm{SU}(2)$ isospin group can be used as an example without any loss of generality as the procedure is equivalent for an arbitrary group of $N$ dimensions. The Lagrangian given in Equation 2.1 is to be modified to account for the different group structure. The Lagrangian for a free isodoublet is

$$
\mathcal{L}=\bar{\psi}(x)\left(i \gamma^{\mu} \partial_{\mu}-m\right) \psi(x)
$$


where $\psi(x)$ is an $\mathrm{SU}(2)$ doublet of complex scalar fields. $\mathrm{SU}(2)$ is a dimension 3 group and hence has three generators. In the $2 \times 2$ matrix representation the generators $T_{a}(a=1,2,3)$ are given by $1 / 2$ times the Pauli spin matrices $\tau_{a}$ :

$$
\begin{aligned}
& T_{1}=\frac{1}{2} \tau_{1}=\frac{1}{2}\left(\begin{array}{cc}
0 & 1 \\
1 & 0
\end{array}\right), \\
& T_{2}=\frac{1}{2} \tau_{2}=\frac{1}{2}\left(\begin{array}{cc}
0 & -i \\
i & 0
\end{array}\right), \\
& T_{3}=\frac{1}{2} \tau_{3}=\frac{1}{2}\left(\begin{array}{cc}
1 & 0 \\
0 & -1
\end{array}\right),
\end{aligned}
$$

and obey the following commutation relations:

$$
\left[T_{a}, T_{b}\right]=i \epsilon_{a b c} T_{c}
$$

Hence the gauge transformation for a field $\psi(x)$ is

$$
\psi(x) \rightarrow \psi^{\prime}(x)=e^{i \boldsymbol{\alpha}(x) \cdot \boldsymbol{T}} \psi(x),
$$

where $\boldsymbol{\alpha}(x)$ are analogous to the real space-time fields $\alpha(x)$ in the Abelian case. To achieve local SU(2) invariance of $\mathcal{L}$, the partial derivative $\partial_{\mu}$ is replaced by the covariant derivative:

$$
D_{\mu}=\partial_{\mu}+i g \boldsymbol{T} \cdot \boldsymbol{W}_{\mu}(x),
$$

where three gauge fields $\boldsymbol{W}_{\mu}$ join the cast. Under an infinitesimal gauge fields transform as

$$
\psi(x) \rightarrow \psi^{\prime}(x)=(1+i \boldsymbol{\alpha}(x) \cdot \boldsymbol{T}) \psi(x),
$$

the three gauge fields transform as

$$
\boldsymbol{W}_{\mu}(x) \rightarrow \boldsymbol{W}_{\mu}(x)-\frac{1}{g} \partial_{\mu} \boldsymbol{\alpha}(x)-\boldsymbol{\alpha}(x) \times \boldsymbol{W}_{\mu}(x) .
$$

The gauge invariant Lagrangian is then

$$
\mathcal{L}=\bar{\psi}(x)\left(i \gamma^{\mu} D_{\mu}-m\right) \psi(x)-\frac{1}{4} \boldsymbol{W}_{\mu \nu} \times \boldsymbol{W}^{\mu \nu},
$$

where the kinetic energy term of the gauge fields are added with

$$
\boldsymbol{W}_{\mu \nu}=\partial_{\mu} \boldsymbol{W}_{\nu}(x)-\partial_{\nu} \boldsymbol{W}_{\mu}(x)-g \boldsymbol{W}_{\mu}(x) \times \boldsymbol{W}_{\nu}(x) .
$$

The last terms in Equation 2.17 and Equation 2.19 arise from the non-Abelian character of the group.

\subsection{Electroweak Theory}

The Standard Model neatly groups the experimentally observed elementary particles of the Universe [7]. It describes the strong and the electroweak interactions of all 


\begin{tabular}{|c|c|c|c|c|c|c|}
\hline & \multicolumn{3}{|c|}{ Family } & \multicolumn{3}{|c|}{ Quantum Number } \\
\hline & 1 & 2 & 3 & $T^{3}$ & $Y$ & $Q$ \\
\hline \multirow{3}{*}{ Leptons } & & $\nu_{\mu}$ & $\bar{\nu} \nu_{\tau}$ & $1 / 2$ & $-1 / 2$ & 0 \\
\hline & $e^{-}$ & $\mu^{-}$ & $\tau^{-}$ & $-1 / 2$ & $-1 / 2$ & -1 \\
\hline & $e_{R}$ & $\mu_{R}$ & $\tau_{R}$ & 0 & -1 & -1 \\
\hline \multirow{4}{*}{ Quarks } & & & $t$ & $1 / 2$ & $1 / 6$ & $2 / 3$ \\
\hline & $\left.d^{\prime}\right)$ & $\left.s^{\prime}\right)$ & $b^{\prime}$ & $-1 / 2$ & $-1 / 6$ & $-1 / 3$ \\
\hline & $u_{R}$ & $c_{R}$ & $t_{R}$ & 0 & $2 / 3$ & $2 / 3$ \\
\hline & $d_{R}$ & $s_{R}$ & $b_{R}$ & 0 & $-1 / 3$ & $-1 / 3$ \\
\hline
\end{tabular}

Table 2.1: Multiplet and quantum number assignments for the fermions in the Standard Model. The prime indicates that the weak eigenstates of the quarks are not their mass eigenstates. The quark mixing is described by the Cabibbo-KobayashiMaskawa matrix. The indices $L(R)$ denote left (right)-handed fermions.

particles known today. In the following only the electroweak part of the Standard Model is discussed.

The electroweak theory established by Glashow, Salam and Weinberg [1] is a gauge theory which unifies the electromagnetic and the weak force. Its importance is based on the successful description and ordering of particles and their properties which were observed in experiments. One of the most striking and convincing arguments in favor of this theory was the prediction of the heavy gauge bosons $W$ and $Z^{0}$. The observation of weak neutral current reactions by the Gargamelle Neutrino Collaboration in 1973 can be considered as a milestone for the manifestation of the Glashow-Salam-Weinberg model as a standard theory [8]. Eventually the discovery of $W$ and $Z^{0}$ bosons in 1983 was the breakthrough of the model [9]. The electroweak theory is a non-Abelian theory based on the $\mathrm{SU}(2) \times \mathrm{U}(1)$ group. The generators of the group are the three components of the weak isospin $\boldsymbol{T}$ and the hypercharge $Y$. They are related to the electromagnetic charge in the following way:

$$
Q=T^{3}+Y
$$

Parity violation is introduced by grouping left-handed and right-handed particles in different weak isospin multiplets. Left-handed fermions are grouped in doublets, whereas right-handed fermions are singlets. Table 2.1 summarizes the fermions of the Standard Model. Requiring invariance of the Lagrangian under a local SU(2) $\times \mathrm{U}(1)$ gauge transformation leads to the introduction of four gauge fields $\boldsymbol{W}_{\mu}$ and $B_{\mu}$ with the covariant derivative:

$$
D_{\mu} \equiv \partial_{\mu}+i g \boldsymbol{T} \cdot \boldsymbol{W}_{\mu}+i g^{\prime} \frac{Y}{2} B_{\mu}
$$

where $g$ and $g^{\prime}$ are the coupling constants of the interactions.

The Lagrangian of the electroweak theory can then be written as the sum of four independent terms:

$$
\mathcal{L}=\mathcal{L}_{\text {Fermion }}+\mathcal{L}_{\text {Gauge }}+\mathcal{L}_{\text {Higgs }}+\mathcal{L}_{\text {Yukawa }}
$$


where $\mathcal{L}_{\text {Fermion }}$ describes massless fermion fields and their interaction with the gauge fields:

$$
\mathcal{L}_{\text {Fermion }}=i \bar{\psi} \gamma^{\mu} D_{\mu} \psi
$$

The term $\mathcal{L}_{\text {Gauge }}$ contains the kinetic energy of the massless gauge fields $\boldsymbol{W}$ and $B$ and the self interaction of the $\boldsymbol{W}$ fields:

$$
L_{\text {Gauge }}=-\frac{1}{4} \boldsymbol{W}_{\mu \nu} \boldsymbol{W}^{\mu \nu}-\frac{1}{4} B_{\mu \nu} B^{\mu \nu},
$$

where

$$
\begin{aligned}
\boldsymbol{W}_{\mu \nu} & =\partial_{\mu} \boldsymbol{W}_{\nu}-\partial_{\nu} \boldsymbol{W}_{\mu}+g \boldsymbol{W}_{\mu} \times \boldsymbol{W}_{\nu}, \\
B_{\mu \nu} & =\partial_{\mu} B_{\nu}-\partial_{\nu} B_{\mu} .
\end{aligned}
$$

The third term in Equation 2.26 is the self interaction of the $\boldsymbol{W}$ fields. It arises from the non-Abelian character of the $\mathrm{SU}(2)$ group. The term $\mathcal{L}_{\text {Higgs }}$ and $\mathcal{L}_{\text {Yukawa }}$ describe the interaction of the gauge and the fermion fields with the Higgs boson. The structure of these terms will be discussed in section 2.5.2.

The mass eigenstates of the $W$ bosons are

$$
W_{\mu}^{ \pm}=\frac{1}{\sqrt{2}}\left(W_{\mu}^{1} \mp i W_{\mu}^{2}\right) .
$$

The photon field, $A_{\mu}$, and the field of the $Z^{0}$ boson, $Z_{\mu}$, are linear combination of $B_{\mu}$ and $W_{\mu}^{3}$ :

$$
\left(\begin{array}{c}
A_{\mu} \\
Z_{\mu}
\end{array}\right)=\left(\begin{array}{cc}
\cos \theta_{W} & \sin \theta_{W} \\
-\sin \theta_{W} & \cos \theta_{W}
\end{array}\right)\left(\begin{array}{c}
B_{\mu} \\
W_{\mu}^{3}
\end{array}\right)
$$

The weak mixing angle $\theta_{W}$ depends on the coupling constants $g$ and $g^{\prime}$ :

$$
\begin{aligned}
& \sin \theta_{W}=\frac{g^{\prime}}{\sqrt{g^{2}+g^{\prime 2}}}, \\
& \cos \theta_{W}=\frac{g}{\sqrt{g^{2}+g^{\prime 2}}} .
\end{aligned}
$$

So far, the gauge bosons, which were naturally introduced by requiring $\mathrm{SU}(2) \times$ $\mathrm{U}(1)$ gauge invariance, are massless. However, the $Z^{0}$ and $W$ bosons as well as the fermions are massive. Mass terms such as $(1 / 2) m^{2} B_{\mu} B^{\mu}$ for bosons and $m \bar{\psi} \psi$ for fermions would destroy the gauge invariance of the Lagrangian. Therefore, the mass is introduced in a very specific way, which is discussed in the next section.

\subsection{The Higgs Mechanism}

The Higgs mechanism [4] is introduced to give masses to the $W$ and $Z^{0}$ bosons, while keeping the invariance under a local $\mathrm{SU}(2) \times \mathrm{U}(1)$ gauge transformation. The same mechanism can be used to generate the fermion masses. In this section the general idea of spontaneous symmetry breaking and the mass generation of the gauge bosons and fermions are discussed. 


\subsubsection{Spontaneous Symmetry Breaking}

We assume a scalar field with the potential:

$$
V(\Phi)=\frac{1}{2} \mu^{2} \Phi^{2}+\frac{1}{4} \lambda \Phi^{4},
$$

with $\mu^{2}<0$ and $\lambda>0$. A potential, defined in this way, has one maximum value at $\Phi=0$ and two minimum values at $\Phi= \pm v$, where

$$
v=\sqrt{\frac{-\mu^{2}}{\lambda}}
$$

The ground state $v$ is called the vacuum state. Figure 2.1 shows the potential. Such a potential, where the vacuum state does not have the same symmetry as the

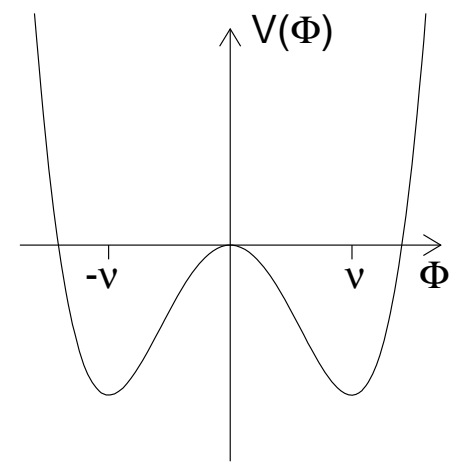

Figure 2.1: Potential $V(\Phi)=(1 / 2) \mu^{2} \Phi^{2}+(1 / 4) \lambda \Phi^{4}\left(\mu^{2}<0\right.$ and $\left.\lambda>0\right)$.

potential itself, is called spontaneously broken. Or in other words, the symmetry is hidden by the choice of the vacuum state.

Particles are quantum excitations of the vacuum state. Therefore, one can construct the particle spectrum by expanding the field $\Phi$ around the vacuum state:

$$
\Phi(x)=v+h(x) .
$$

The Lagrangian of the scalar Higgs field

$$
\mathcal{L}=\frac{1}{2} \partial_{\mu} \Phi \partial^{\mu} \Phi-\left(\frac{1}{2} \mu^{2} \Phi^{2}+\frac{1}{4} \lambda \Phi^{4}\right),
$$

then becomes

$$
\mathcal{L}=\frac{1}{2} \partial_{\mu} h \partial^{\mu} h-\left(\lambda v^{2} h^{2}+\lambda v h^{3}+\frac{1}{4} \lambda h^{4}\right)+\text { const. }
$$

The obvious symmetry $\Phi \rightarrow-\Phi$ of the Lagrangian given by Equation 2.34 is well hidden in Equation 2.35, although the two representations are completely equivalent. The term proportional to $h^{2}$ is interpreted as a mass term with

$$
m_{h}^{2}=2 \lambda v^{2}=-2 \mu^{2},
$$

whereas the higher order terms of $h$ are self-interaction terms of the $h$ field. 


\subsubsection{The Higgs Boson in the Standard Model}

In the last section a mass for a spin-0 particle was created by choosing a non-zero vacuum expectation value, which breaks spontaneously the symmetry of a Lagrangian that was invariant under a $\Phi \rightarrow-\Phi$ transformation.

In the Standard Model we require the gauge invariance under a local $\mathrm{SU}(2) \times$ $\mathrm{U}(1)$ transformation. Massive gauge bosons are needed to explain the "weakness" of interactions at low energies. The minimal model which can generate these masses without violating the gauge invariance of the Lagrangian is an one doublet Higgs model. In this model the Higgs boson is introduced as a weak isospin doublet:

$$
\Phi=\left(\begin{array}{c}
\Phi^{+} \\
\Phi^{0}
\end{array}\right)
$$

with the complex fields

$$
\begin{aligned}
& \Phi^{+}=\frac{\Phi_{1}+i \Phi_{2}}{\sqrt{2}}, \\
& \Phi^{0}=\frac{\Phi_{3}+i \Phi_{4}}{\sqrt{2}} .
\end{aligned}
$$

The Higgs potential is now

$$
V(\Phi)=\mu^{2} \Phi^{\dagger} \Phi+\lambda\left(\Phi^{\dagger} \Phi\right)^{2}
$$

Remembering, that for keeping the local $\mathrm{SU}(2) \times \mathrm{U}(1)$ gauge invariance, the partial derivative $\partial_{\mu}$ must be replaced by the covariant derivative of Equation 2.21, leads to the Lagrangian:

$$
\mathcal{L}_{\text {Higgs }}=\left(D_{\mu} \Phi\right)^{\dagger}\left(D^{\mu} \Phi\right)-\mu^{2} \Phi^{\dagger} \Phi-\lambda\left(\Phi^{\dagger} \Phi\right)^{2}
$$

The minimum of the Higgs potential for $\mu^{2}<0$ are at

$$
\Phi^{\dagger} \Phi=\frac{\Phi_{1}^{2}+\Phi_{2}^{2}+\Phi_{3}^{2}+\Phi_{4}^{2}}{2}=\frac{-\mu^{2}}{2 \lambda}=\frac{v^{2}}{2} .
$$

Choosing one particular ground state $\Phi_{3}=v, \Phi_{1}=\Phi_{2}=\Phi_{4}=0$ and expanding around it, leads to

$$
\Phi(x)=\frac{1}{\sqrt{2}}\left(\begin{array}{c}
0 \\
v+h(x)
\end{array}\right)
$$

One should note that this choice is not random. Choosing only for $\Phi_{3}$ a non-zero vacuum expectation value with setting others to zero, ensures that the electric charge is conserved. A non-zero vacuum expectation value of $\Phi^{+}$would lead to electric charge violation, which is in contradiction to experiment. The $\mathrm{O}(4)$ symmetry of Equation 2.42 has been broken down to an $\mathrm{O}(1)$ symmetry corresponding to the $\mathrm{U}(1)_{\text {EM }}$ group of electromagnetism. Three fields have been "gauged away." They will become the longitudinal polarization states of the $W$ and $Z^{0}$ bosons which are 
needed for massive gauge bosons. Investigating the first term in Equation 2.41 at the vacuum state and inserting $Y=1 / 2$ yields

$$
\begin{aligned}
\mid\left(i g \boldsymbol{T} \cdot \boldsymbol{W}_{\mu}\right. & \left.+i g^{\prime} \frac{Y}{2} B_{\mu}\right)\left.\right|^{2} \\
& =\frac{1}{8} v^{2} g^{2}\left(\left(W_{\mu}^{1}\right)^{2}+\left(W_{\mu}^{2}\right)^{2}\right)+\frac{1}{8} v^{2}\left(g^{\prime} B_{\mu}-g W_{\mu}^{3}\right)^{2} \\
& =\left(\frac{1}{2} v g\right)^{2}\left(W^{+}\right)_{\mu}\left(W^{-}\right)^{\mu}+\frac{1}{2}\left(\frac{1}{2} v \sqrt{g^{2}+g^{\prime 2}}\right)^{2} Z_{\mu} Z^{\mu} .
\end{aligned}
$$

For deriving the last line, the identities given by Equation 2.27 and Equation 2.28 were used. For charged bosons one expects a mass term $m_{W}^{2}\left(W^{+}\right)_{\mu}\left(W^{-}\right)^{\mu}$ and for the neutral bosons $(1 / 2)\left(m_{Z}^{2} Z_{\mu} Z^{\mu}+m_{A}^{2} A_{\mu} A^{\mu}\right)$. Therefore, the masses of the gauge bosons are given by

$$
\begin{aligned}
m_{W} & =\frac{1}{2} v g \\
m_{Z} & =\frac{1}{2} v \sqrt{g^{2}+g^{\prime 2}},
\end{aligned}
$$

and

$$
m_{\gamma}=0
$$

The last equation is derived from the fact that there is no term of the form $A_{\mu} A^{\mu}$ in Equation 2.44. These boson masses relate to the weak mixing angle:

$$
\cos \theta_{W}=\frac{m_{W}}{m_{Z}}
$$

A useful quantity which is often referred to is

$$
\rho=\frac{m_{W}}{m_{Z} \cos \theta} .
$$

As can be seen from Equation 2.48, the Standard Model predicts $\rho=1$ at the tree level. The experimental values of the $m_{W}, m_{Z}$ and $\sin ^{2} \theta_{W}$ have been precisely measured and yield $\rho=0.9998_{-0.0005}^{+0.000}$. Any theory making predictions on the mass generation will have to reproduce these measurements. The Higgs boson mass is predicted to be

$$
m_{H}=\sqrt{2 \lambda} v .
$$

Since $\lambda$ is unknown, it remains a free parameter of the theory. The bosons and their quantum number assignments in the Standard Model are summarized in Table 2.2

The fermion masses are generated by adding a $\mathrm{SU}(2) \times \mathrm{U}(1)$ gauge invariant interaction term of the Higgs boson field with the fermion fields to the Lagrangian. For the first lepton family (and similarly for the other lepton flavors) this is

$$
\mathcal{L}_{\text {Yukawa }}=G_{e}\left[(\bar{\nu}, \bar{e})_{L}\left(\begin{array}{c}
\Phi^{+} \\
\Phi^{0}
\end{array}\right) e_{R}+\bar{e}_{R}\left(\Phi^{-}, \bar{\Phi}^{0}\right)\left(\begin{array}{c}
\nu_{e} \\
e
\end{array}\right)_{L}\right] .
$$




\begin{tabular}{ccccc}
\hline \hline & & $T^{3}$ & $Y$ & $Q$ \\
\hline \multirow{3}{*}{ Gauge } & $\gamma$ & 0 & 0 & 0 \\
Bosons & $W^{+}$ & +1 & 0 & +1 \\
& $W^{-}$ & -1 & 0 & -1 \\
& $Z^{0}$ & 0 & 0 & 0 \\
\hline \multirow{2}{*}{ Higgs Boson } & \multirow{2}{*}{$\Phi=\left(\begin{array}{c}\Phi^{+} \\
\Phi^{0}\end{array}\right)$} & $+1 / 2$ & $+1 / 2$ & 1 \\
& & $-1 / 2$ & $+1 / 2$ & 0 \\
\hline \hline
\end{tabular}

Table 2.2: Quantum number assignments of the bosons in the Standard Model.

After spontaneous symmetry breaking, one obtains

$$
\mathcal{L}_{\text {Yukawa }}=\frac{G_{e}}{\sqrt{2}} v\left(\bar{e}_{L} e_{R}+\bar{e}_{R} e_{L}\right)+\frac{G_{e}}{\sqrt{2}}\left(\bar{e}_{L} e_{R}+\bar{e}_{R} e_{L}\right) h
$$

from which we can conclude that the electron mass is

$$
m_{e}=\frac{G_{e} v}{\sqrt{2}},
$$

whereas the neutrino remains massless. One should note that the coupling constant $G_{e}$ is not determined by the theory. However from Equation 2.53 it can be seen that it is proportional to the electron mass. Quark masses can be generated similarly. In order allow masses for up-type quarks the charge-conjugate Higgs doublet is used:

$$
\Phi_{c}=-i \tau_{2} \Phi^{*}=\left(\begin{array}{c}
-\bar{\Phi}^{0} \\
\Phi^{-}
\end{array}\right)
$$

The Yukawa term then becomes

$$
\begin{aligned}
\mathcal{L}_{\text {Yukawa }}= & G_{d}\left[(\bar{u}, \bar{d})_{L}\left(\begin{array}{c}
\Phi^{+} \\
\Phi^{0}
\end{array}\right) d_{R}+\bar{d}_{R}\left(\Phi^{-}, \bar{\Phi}^{0}\right)\left(\begin{array}{c}
u \\
d
\end{array}\right)_{L}\right] \\
+ & G_{u}\left[(\bar{u}, \bar{d})_{L}\left(\begin{array}{c}
-\bar{\Phi}^{0} \\
\Phi^{-}
\end{array}\right) d_{R}+\bar{d}_{R}\left(-\Phi^{0}, \bar{\Phi}^{+}\right)\left(\begin{array}{c}
u \\
d
\end{array}\right)_{L}\right] .
\end{aligned}
$$

After spontaneous symmetry breakdown this equation yields

$$
\mathcal{L}_{\text {Yukawa }}=\frac{G_{d} v}{\sqrt{2}} \bar{d} d+\frac{G_{u} v}{\sqrt{2}} \bar{u} u+\frac{G_{d}}{\sqrt{2}} \bar{d} d h+\frac{G_{u}}{\sqrt{2}} \bar{u} u h,
$$

from which the down and up quark masses $G_{d} v / \sqrt{2}$ and $G_{u} v / \sqrt{2}$ are derived. In general the mass of any given fermion $f$ is proportional to the coupling constant $G_{f}$ to the Higgs boson:

$$
m_{f}=\frac{G_{f} v}{\sqrt{2}} .
$$

This proportionality predicts that the Higgs boson prefers to decay into the heavier fermions - a property which is used in presently ongoing searches. 


\subsection{The Higgs Boson Production and Decay at the Tevatron}

After having introduced the principal idea and the properties of the Higgs boson in the Standard Model, the possible production scenario at the $p \bar{p}$ collider Tevatron is discussed in this section. The Tevatron is an accelerator, where the beam energy is $980 \mathrm{GeV}$. Currently the Tevatron is the only active facility capable of probing the Higgs boson.

\subsubsection{The Higgs Boson Production}

At the Tevatron there are three primary production modes of interest for the Standard Model Higgs boson. The main problems in the Higgs searches at the Tevatron are related to the hadronic environment. This creates problems for the event triggering to dictate which Higgs production mechanisms and decay channels are to be used.

Figure 2.2 shows the production cross section of the Higgs boson as a function of the mass at the Tevatron. The dominant process is gluon-gluon fusion, in which the

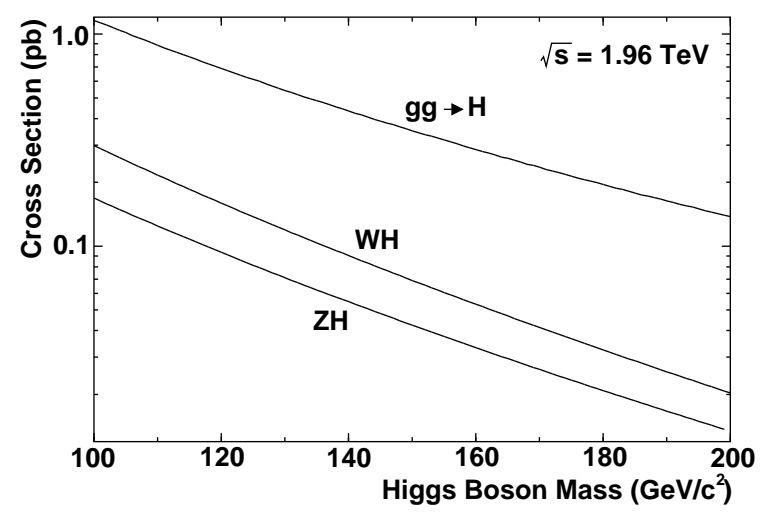

Figure 2.2: The production cross section of the Higgs boson as a function of the mass at the Tevatron.

Higgs boson is produced via a quark loop, shown in Figure 2.3. But this production channel will be difficult to exploit due to the large quantum chromodynamics (QCD) multi-jet backgrounds. Roughly a factor of five lower in the cross section are the

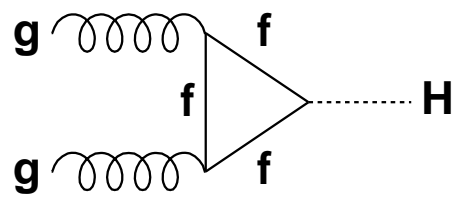

Figure 2.3: The Feynman diagram of gluon-gluon fusion process. 
two associated processes where the Higgs boson is produced in association with a $W$ or $Z^{0}$ boson.

\section{Associated Higgs Boson Production with the $W$ Boson}

At the Tevatron the process $q \bar{q} \rightarrow W H$ is the most promising to tag the Higgs boson by the $W$ boson decay products. This process has the following cross section [10]:

$$
\sigma\left(q_{i}{\overline{q^{\prime}}}_{j} \rightarrow W H\right)=\frac{\pi \alpha^{2}\left|V_{i j}\right|^{2}}{36 \sin ^{4} \theta_{W}} \cdot \frac{2 K}{\sqrt{s}} \cdot \frac{K^{2}+3 M_{W}^{2}}{\left(s-M_{W}^{2}\right)^{2}},
$$

where $\alpha\left(m_{Z}\right)=e^{2} / 4 \pi \hbar c(\sim 1 / 128),\left|V_{i j}\right|$ is one of the elements of the CabibboKobayashi-Maskawa matrix associated with the $q_{i} \bar{q}^{\prime}{ }_{j} \rightarrow W$ vertex [11], $\sqrt{s}$ is the center of mass energy of the $q_{i} \bar{q}^{\prime}{ }_{j}$ collision, $K$ is the center of mass momentum of the produced Higgs boson and $M_{W}$ is the $W$ boson mass. Since this process has a relatively small cross section, it is important to compute the rate as accurately as possible by including the QCD radiative corrections. The higher order QCD corrections produce the production cross section shown in Figure 2.2. The $W H$ events can be tagged by identifying the charged lepton from the $W$ decay. Imposing the isolation cuts on the lepton significantly reduces the background. For $m_{H}=115$ $\mathrm{GeV} / c^{2}$, where $m_{H}$ is the Higgs boson mass, the production cross section is about $0.2 \mathrm{pb}$.

\subsubsection{The Higgs Boson Decay}

The profile of the Higgs boson is uniquely determined if the Higgs boson mass is fixed [12]. Figure 2.4 shows the branching ratio of the Higgs boson. The main decay modes are decays into $b \bar{b}, W^{+} W^{-}$and $Z^{0} Z^{0}$ pairs, one of the gauge bosons being virtual below the respective threshold. Above the $W^{+} W^{-}$and $Z^{0} Z^{0}$ pair thresholds, the Higgs bosons decay almost exclusively into these two channels, with an admixture of $t$ quark decays near and above the $t \bar{t}$ threshold. Below $m_{H}=140$ $\mathrm{GeV} / c^{2}$, the decays $H \rightarrow \tau^{+} \tau^{-}, c \bar{c}$ and $g g$ are also important besides the dominating $b \bar{b}$ channel. Though suppressed in rate, $H \rightarrow \gamma \gamma$ provides a clear 2-body signature for the formation of Higgs bosons.

\section{Higgs Boson Decays into Fermions}

The dominant decay of a Higgs boson with a mass below the $W^{+} W^{-}$threshold are into fermion-antifermion pairs. The partial decay width of the Higgs boson to fermions is given by the following equation [13]:

$$
\Gamma(H \rightarrow f \bar{f})=\mathcal{N}_{c} \frac{G_{F} m_{f}^{2}}{4 \sqrt{2} \pi} m_{H} \beta_{f}^{3},
$$

where $\mathcal{N}_{c}$ is the color factor $\left(\mathcal{N}_{c}=1\right.$ for leptons and 3 for quarks $), G_{F}$ is the Fermi constant, $\beta_{f}=\sqrt{1-4 m_{f}^{2} / m_{H}^{2}}$ is the velocity of the final state fermion in the Higgs 


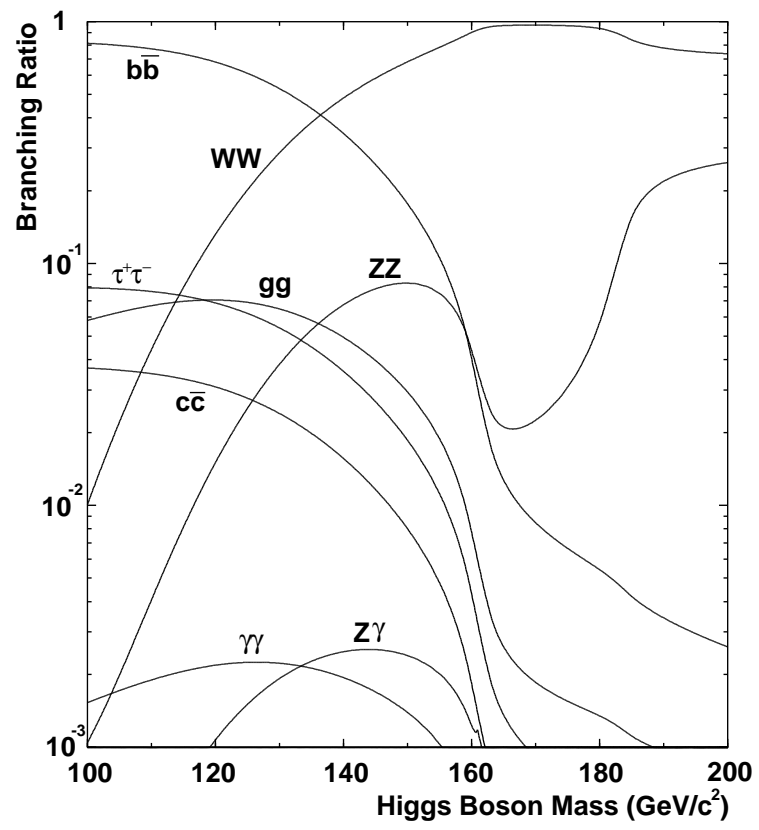

Figure 2.4: The branching ratio of the Higgs boson as a function of the mass.

boson rest frame. Higher order QCD corrections produce the branching ratios shown in Figure 2.4. The partial width is proportional to the square of the fermion mass. In the Tevatron region we expect the Higgs boson to decay most strongly to the kinematically available heaviest fermions, in this case the $b$ quarks.

\subsubsection{Main Search Channels at Tevatron}

The Higgs bosons are produced through a variety of mechanisms at the Tevatron. Figure 2.5 shows some Feynman diagrams of the Higgs boson productions which are promising channels at the Tevatron.

In this thesis, we search for the Higgs boson using $p \bar{p} \rightarrow W H \rightarrow \ell \nu b \bar{b}$, and this channel is the most promising channel at the Tevatron. Despite a smaller cross section than $g g \rightarrow H$, this production offers a signature whose backgrounds are considerably more manageable. The decay products of the leptonically decaying $W$ boson offers an additional handle for event identification ${ }^{1}$. The presence of a high $p_{T}$ lepton from $W$ decay can provide a clean trigger for baseline event selection. The neutrino that is produced in the leptonic $W$ decay escapes the detector without leaving telltale interaction remnants in their wake: however these neutrinos do carry away some of energy from the incident collision. The presence of neutrinos is inferred by an energy imbalance in the final state.

The $W$ boson decays to all three lepton families. We look for the decays $W \rightarrow$ $e \nu$ and $W \rightarrow \mu \nu$ in this analysis; $W \rightarrow \tau \nu$ is not considered here, because the

\footnotetext{
${ }^{1}$ The hadronic $W$ decay mode is avoided, because it leads to a four jet final state, $W H \rightarrow q \overline{q^{\prime}} b \bar{b}$. Like gluon-gluon fusion, this four jet final state also has formidable QCD backgrounds that we seek to avoid.
} 
large branching ratio $(\sim 64 \%)$ of the $\tau$ lepton to hadronic final states makes $\tau$ identification considerably more difficult. However, instances of $W \rightarrow \tau \nu$ in which the $\tau$ decays to leptons $(\sim 36 \%)$ are within the acceptance of the analysis.

In summary, the channel which we investigate in this analysis is $W H \rightarrow \ell \nu b \bar{b}$, where $\ell$ is an electron or muon. We are not free however from the influence of background channels in this final state: prominent background channels include $W+$ jets production, $t \bar{t}$ production, single top production and diboson channels.

\subsection{Review of the Previous Higgs Boson Search}

\subsubsection{Direct Search at LEP}

The LEP experiments performed a direct search for the Higgs boson using 2461 $\mathrm{pb}^{-1}$ of data at the center of mass energies between 189 and $209 \mathrm{GeV}$ [5]. At LEP the search channels were $e^{+} e^{-} \rightarrow Z^{0} H$, where $Z^{0}$ boson decays into all possible channels and Higgs boson decays into $b \bar{b}$. Also the $\tau$ decays of the Higgs with the $Z^{0}$ boson decaying into quarks was used. Figure 2.6 shows the reconstructed dijet mass distributions. Since there is no significant mass peak which is expected from the Higgs boson, the following lower mass limit is established:

$$
m_{H}>114.4 \mathrm{GeV} / c^{2},
$$

at $95 \%$ confidence level (C.L.).

\subsubsection{Indirect Search}

The existence of the Higgs boson has an impact on the value of the most electroweak parameters via higher order loop corrections. Indeed the theory is only renormalizable in the presence of the Higgs boson. One way to place a limit on the Higgs boson mass is to measure the effect of loop corrections to e.g. the $W$ boson mass $\left(m_{W}\right)$ which is logarithmic in the Higgs boson mass. The $W$ boson mass also has a noticeable correction due to $t$ quark loops which is quadratic in the mass of the $t$ quark $\left(m_{t}\right)$. Hence, by accurate measurements of the masses of the $t$ quark and $W$ boson, one can infer the Higgs boson mass, if the Higgs boson exists. Figure 2.7 shows the Standard Model relationship for $m_{W}$ and $m_{t}$ as a function of the Higgs mass [6]. The contours correspond to the measured mass values at a $68 \%$ C.L. Other electroweak parameters also have a similar dependence on the $W$ boson and $t$ quark masses and can be combined to perform an overall fit for the Higgs mass. Figure 2.8 shows the fit result. It can be noted that they point to a light Higgs boson. The electroweak fits place the following mass and upper limit [6]:

$$
\begin{aligned}
& m_{H}=129_{-49}^{+74} \mathrm{GeV} / c^{2} \\
& m_{H}<285 \mathrm{GeV} / c^{2} \text { at } 95 \% \text { C.L. }
\end{aligned}
$$




\subsubsection{Direct Search at the Tevatron Run 1}

Searches for the Higgs boson were performed by the CDF collaboration at the Tevatron during Run 1 experiment [14]. The data sample was about $110 \mathrm{pb}^{-1}$ at the center of mass energy of $1.8 \mathrm{TeV}$. The channels considered were $p \bar{p} \rightarrow V H \rightarrow X+b \bar{b}$, where $V$ was either $W$ or $Z^{0}$ and $X$ included the majority of the possible $W$ and $Z^{0}$ boson decays. The results were expressed as $95 \%$ exclusion curves for the $V H$ cross sections in the various decay channels. The results are shown in Figure 2.9. The upper limits on the cross sections are an order of magnitude above the Standard Model predictions.

\subsubsection{Direct Search at the Tevatron Run 2}

In Run 2 the DØ collaboration has been searching for the Higgs boson at Tevatron [15]. Using $174 \mathrm{pb}^{-1}$ of the integrated luminosity, the DØ collaboration set a $95 \%$ C.L. upper limit. Figure 2.10 shows the upper limit. The upper limits on $W H \rightarrow$ $e \nu b \bar{b}$ production are $9.0 \sim 12.2 \mathrm{pb}$ for the Higgs boson masses of $105 \sim 135 \mathrm{GeV}$. 
(a)

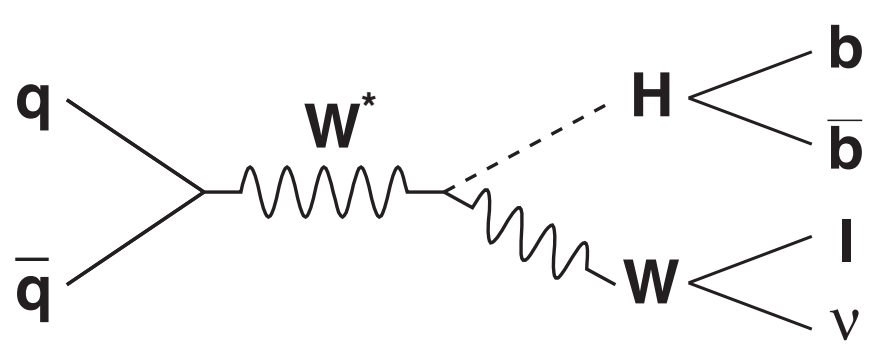

(b)

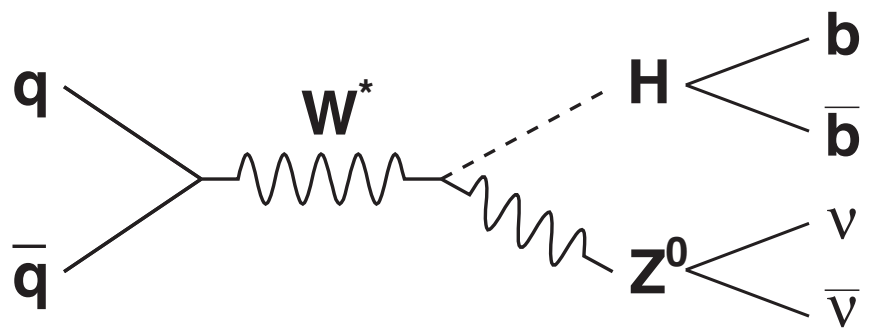

(c)

(d)
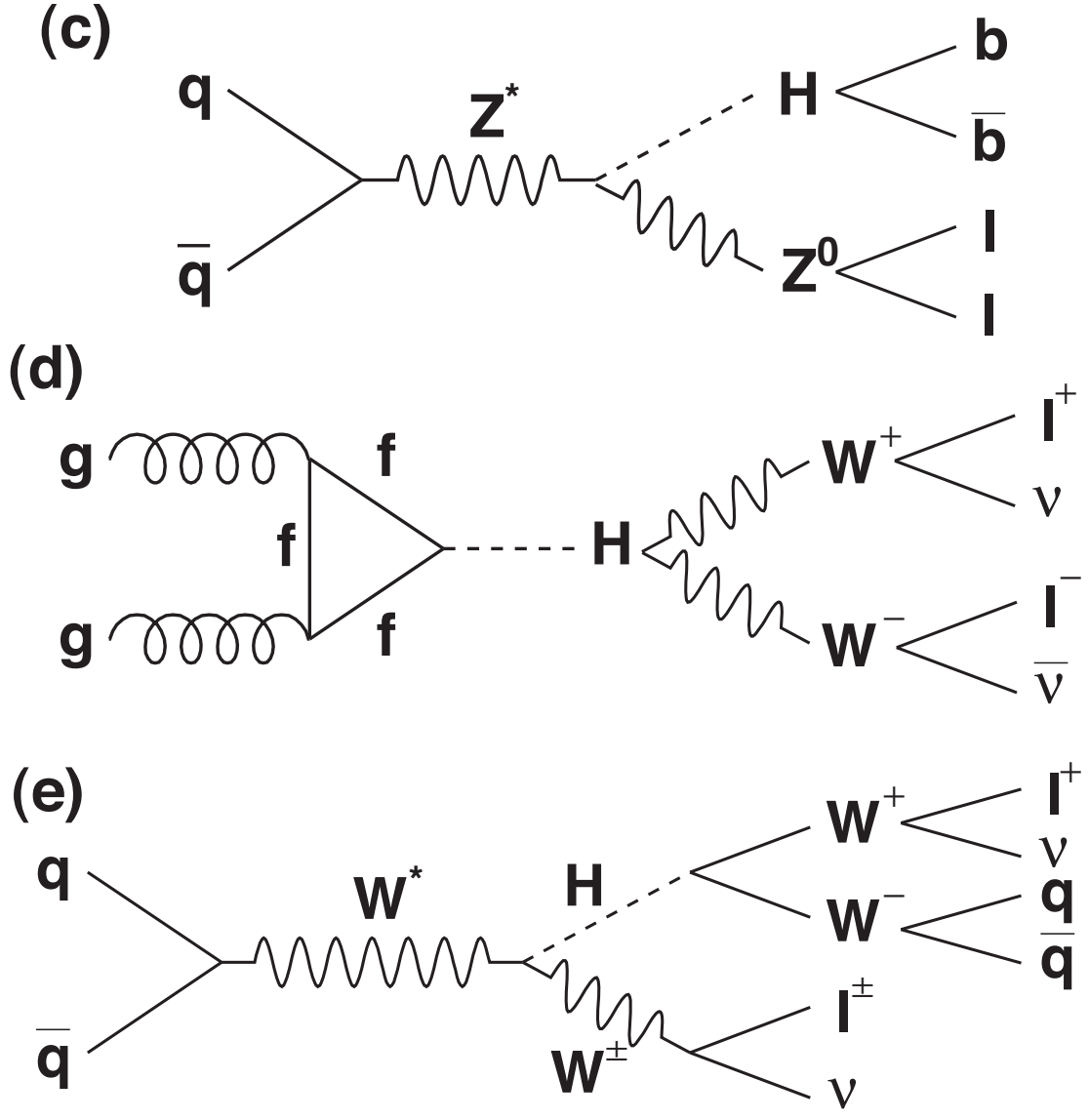

Figure 2.5: The Feynman diagrams of the Higgs boson productions which are promising channels at the Tevatron. (a) $p \bar{p} \rightarrow W H \rightarrow \ell \nu b \bar{b}$, (b) $p \bar{p} \rightarrow Z^{0} H \rightarrow \nu \bar{\nu} b \bar{b}$, (c) $p \bar{p} \rightarrow Z^{0} H \rightarrow \ell^{+} \ell^{-} b \bar{b},(\mathrm{e}) g \bar{g} \rightarrow H \rightarrow W^{+} W^{-} \rightarrow \ell^{+} \nu \ell^{-} \bar{\nu},(\mathrm{d}) p \bar{p} \rightarrow W H \rightarrow$ $W W^{+} W^{-} \rightarrow \ell^{ \pm} \nu \ell^{ \pm} \nu q \bar{q}$. 

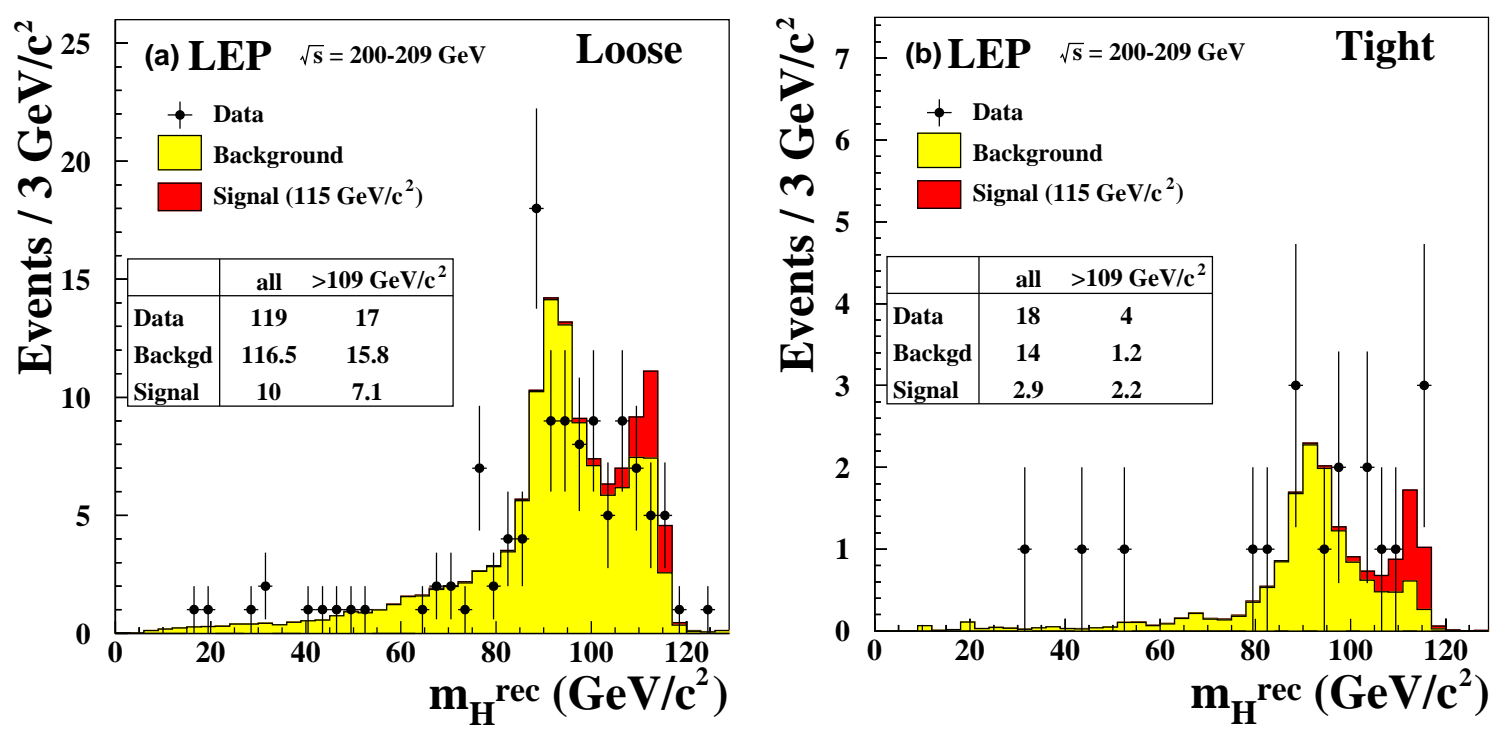

Figure 2.6: Distributions of the reconstructed Higgs boson mass, $m_{H}^{\text {rec }}$, obtained from two selections with different expected signal purities. (a)Loose selections, (b)Tight selections.

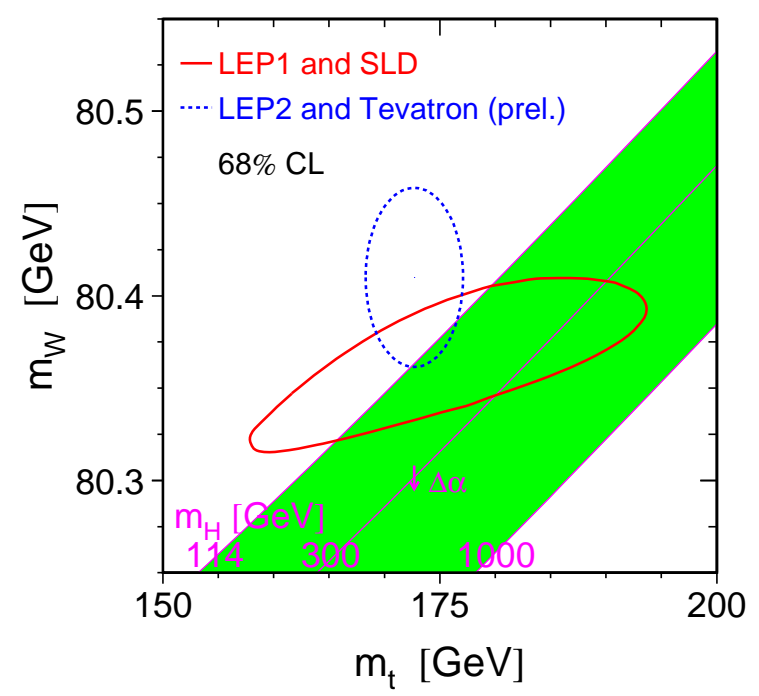

Figure 2.7: The Standard Model relationship for $m_{W}$ and $m_{t}$ as a function of the Higgs mass. The arrow labeled $\Delta \alpha$ shows the variation of this relation if $\alpha\left(m_{Z}^{2}\right)$ is changed by one standard deviation. This variation gives an additional uncertainty to the Standard Model band shown in the figure. 


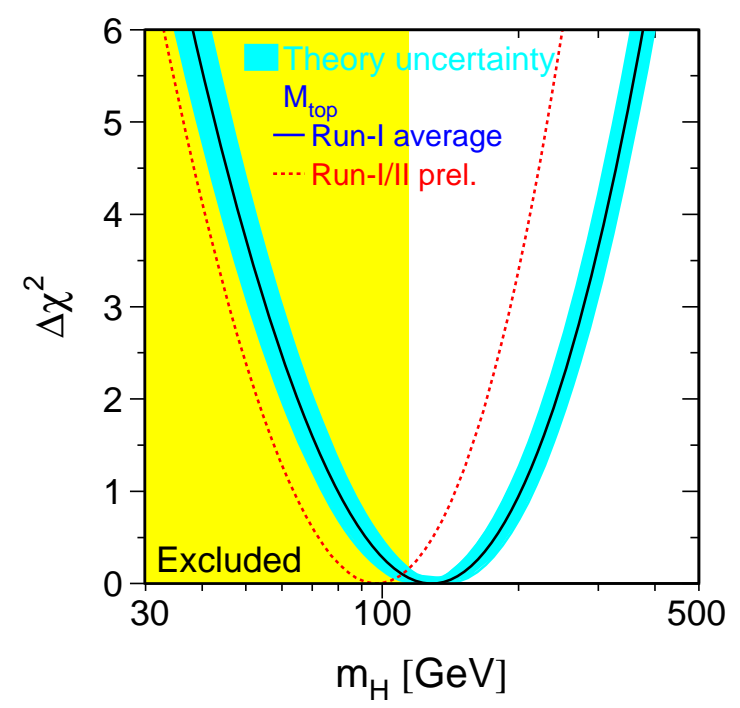

Figure 2.8: The $\Delta \chi^{2}$ distribution as a function of $m_{H}$ from a global fit (with only $m_{H}$ as a free parameter) using the precision electroweak measurements from LEP, SLD, CDF, DØ and NuTeV.

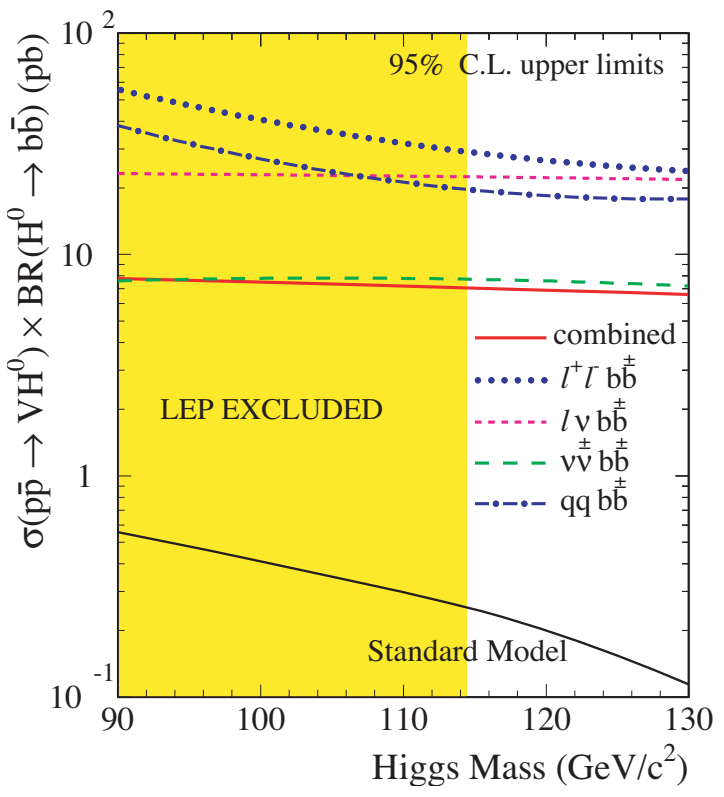

Figure 2.9: The 95\% C.L. upper limits for the Standard Model Higgs boson production cross section from CDF Run 1 data. 


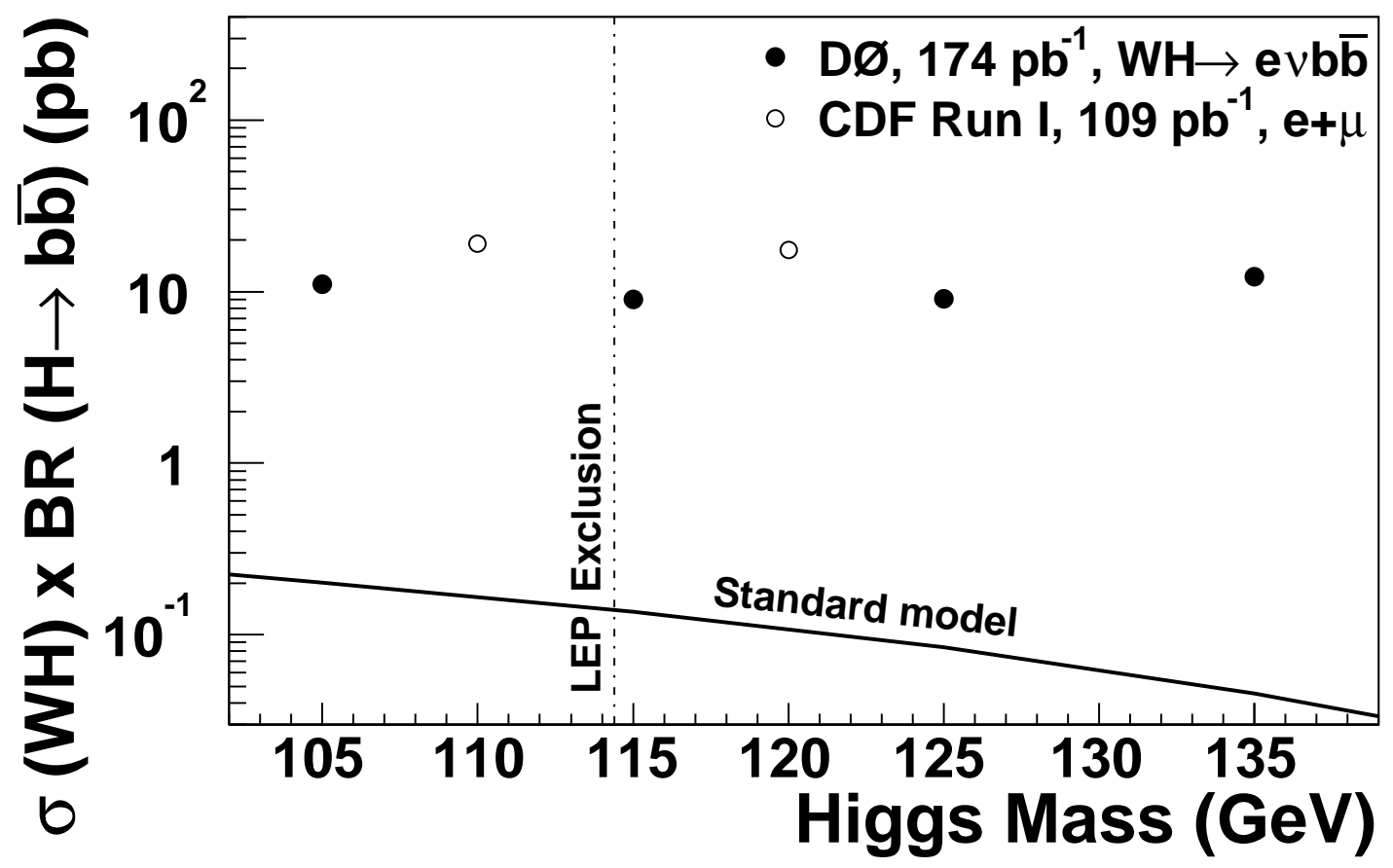

Figure 2.10: The 95\% C.L. upper limits for the Standard Model Higgs boson production cross section from $\mathrm{D} \varnothing$ Run 2 data. 



\section{Chapter 3}

\section{Experimental Apparatus}

The analysis presented in this thesis uses data collected between February 2002 and August 2004 from proton-antiproton collisions produced by the Tevatron at a center-of-mass energy of $1.96 \mathrm{TeV}$, and observed by the Collider Detector at Fermilab $(\mathrm{CDF})$. This chapter describes the collider apparatus and the CDF detector.

\subsection{The Tevatron and the Fermilab Accelerator System}

The Tevatron is a proton-antiproton storage ring complex located at the Fermi National Accelerator Laboratory (Fermilab), in Batavia, Illinois, U.S.A. With a center of mass energy of $\sqrt{s}=1.96 \mathrm{TeV}$, it is currently the most powerful collider, and the only apparatus capable of probing the Higgs boson. The $2 \mathrm{~km}$ diameter storage ring is the last step of a complex chain of accelerators that produce and accelerate the proton and antiproton beams. Figure 3.1 shows the Fermilab accelerator complex. Each step of the accelerator is described in the subsequent sections. Major upgrades have been made to the Fermilab accelerator system in order to increase the luminosity for the Tevatron Run 2. The center of mass energy was also increased from 1.8 TeV to $1.96 \mathrm{TeV}$. Two technical challenges limit the luminosity: the antiproton production and the control of beams of very high intensity. To cope with these challenges, the Main Injector (MI) and the Recycler were built.

\subsubsection{Proton Injector}

The proton beam is initiated with di-hydrogen molecules $\left(\mathrm{H}_{2}\right)$ that are split by an intense local electrostatic field; the resulting $\mathrm{H}^{-}$anions are accelerated by a 750 $\mathrm{kV}$ Cockcroft-Walton machine. The continuous beam of $\mathrm{H}^{-}$ions is segmented into bunches, and transfered into a $150 \mathrm{~m}$ long linear accelerator (LINAC) and brought to an energy of $400 \mathrm{MeV}$. Upon exiting the LINAC, the anions pass through a thin foil of graphite that tears apart the pair of electrons of each ion, leaving only the proton and thus converting the anion beam into a proton beam. The beam is transfered into a $475 \mathrm{~m}$ long circular synchrotron (the Booster) that accelerates the 


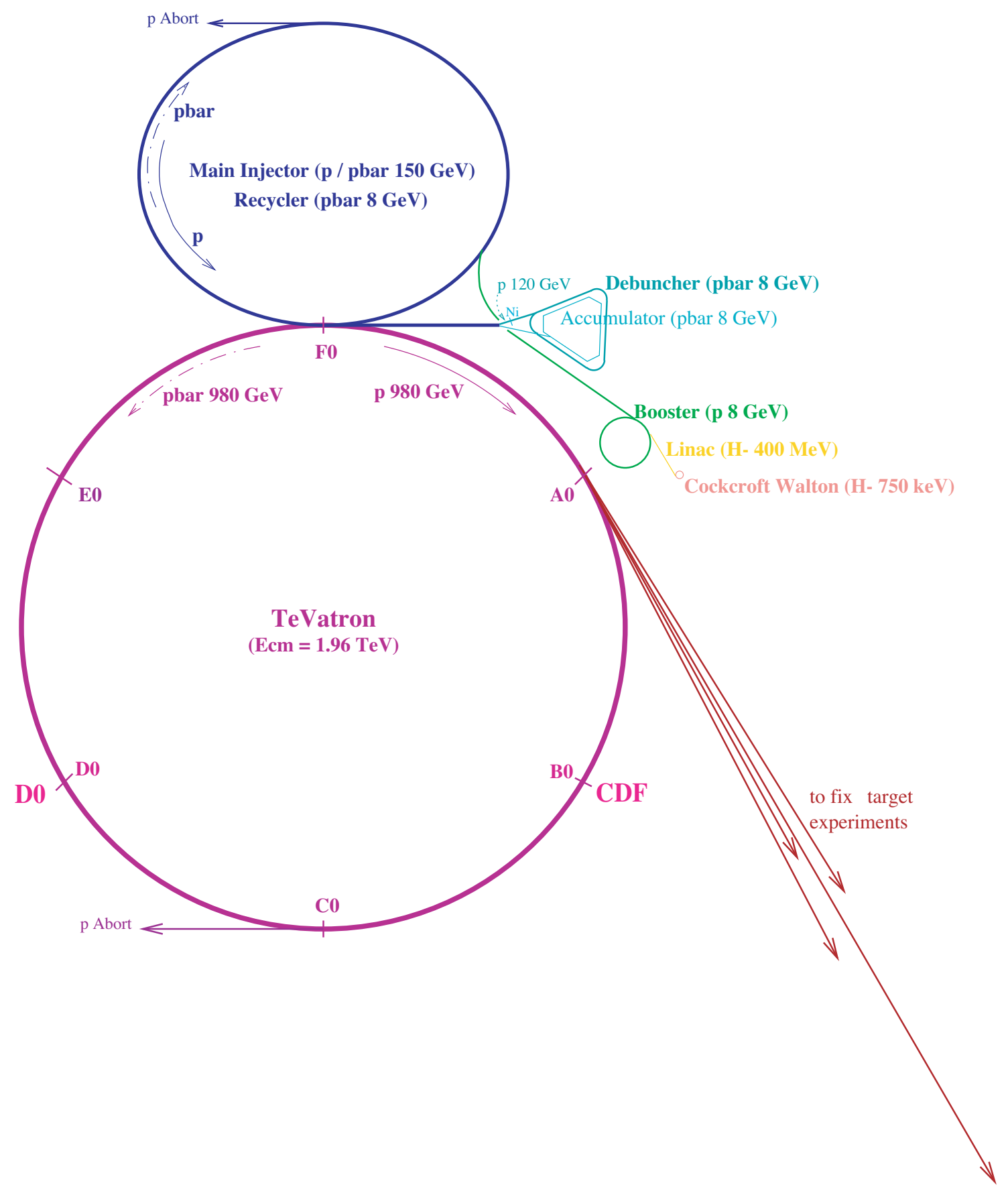

Figure 3.1: The chain of accelerators at Fermilab. 
protons to $8 \mathrm{GeV}$, which are in turn transfered into the MI. The MI is used both for accelerating and injecting the proton beam into the Tevatron and for producing an intense proton beam used to produce antiprotons (see next section). Before injecting into the Tevatron, protons are accelerated up to $150 \mathrm{GeV}$ within a few seconds.

\subsubsection{Antiproton Production and Recycling}

Antiprotons $(\bar{p})$ are produced by colliding a $120 \mathrm{GeV}$ proton beam from the MI onto a Nickel target. Roughly, one antiproton is produced for every million proton collisions. Hence the interaction products need to be efficiently filtered in order to reject non- $\bar{p}$ particles. In a first step, charged particles are focused by a "lithium lens," a tubular piece of lithium traversed by a $650 \mathrm{kA}$ current. Then a pulsed magnet vetoes positively charged particles and selects negative particles with a momentum of the order of $8 \mathrm{GeV}$. After the non- $\bar{p}$ particles decay in flight, only $\bar{p}$ remain. The resulting $\bar{p}$ beam is de-bunched and focused further through stochastic cooling and momentum cooling. Antiprotons are stored into the accumulator, a set of concentric synchrotrons $80 \mathrm{~m}$ long in circumference. Accumulation rates of the order of $10^{11}$ antiprotons per hour have been reached. Once enough antiprotons are accumulated (typically $150 \cdot 10^{10}$ ), they are transfered into the MI in the direction opposite to the protons, accelerated up to $150 \mathrm{GeV}$ together with the proton beam, and transfered into the Tevatron.

Because $\bar{p}$ production is one of the limiting factors to increasing the luminosity, the recycler was designed to recoup the antiprotons that remain in the Tevatron at the end of a store, and re-inject them in the Tevatron for the next store, together with a new stack of antiprotons from the accumulator.

\subsubsection{The Tevatron Storage Ring}

Unlike the other accelerators in the chain, the Tevatron is equipped with super conducting magnets. The beams in the Tevatron have a $36 \times 36$ bunch structure, with a 396 ns time spacing between bunches. At the beginning of a store, the Tevatron beams contain about $10^{13}$ protons and $10^{12}$ antiprotons.

The luminosity is given by the following formula:

$$
\mathcal{L}=\frac{N_{p} N_{\bar{p}} B f}{4 \pi \sigma_{x} \sigma_{y}},
$$

where $N_{p}\left(N_{\bar{p}}\right)$ is the number of protons (antiprotons) per bunch, $B$ is the number of bunches, $f$ is the revolution frequency, and $\sigma_{x}$ and $\sigma_{y}$ are the beam dimensions in the plane transverse to the beam at the interaction point. However, luminosity is not determined from this formula, but from the measured rate of some reference physical processes. The measurement of the luminosity delivered by the Tevatron to the CDF experiments is described in Section 3.2.7. The peak luminosities above $10^{32} \mathrm{~cm}^{-2} \mathrm{~s}^{-1}$ have been reached.

Beams are brought into collision in the center of the CDF and D $\varnothing$ detectors, where the resulting events are recorded. 


\subsection{The Collider Detector at Fermilab}

The Collider Detector at Fermilab (CDF) is a multipurpose high energy particle detector with approximate cylindrical and forward-backward mirror symmetry with respect to the beam axis. The CDF collaboration gathers more than 700 physicists and 49 institutions from 12 different countries. In normal operations, several dozens of physicists and technicians take shifts to run, monitor and maintain the very complex detector. It is composed of several coaxial sub-detectors which serve different purposes, and which will be described in the following sections: the Silicon Vertex Detector and the Central Outer Tracker, which lie inside a 1.4 Tesla superconducting solenoid magnet, form the tracking system, and precisely measure trajectories and momenta of charged particles as they go through the detector; the calorimeters lie outside the solenoid and measure particle energies; the muon system lies outside the calorimeter and detects muons that escape the calorimeters; the Cerenkov Luminosity Counter located near the beam measures the rates of interactions for luminosity measurement. A Time Of Flight detector located between the COT and the solenoid and other sub-detectors used to study diffractive physics are not used in this thesis; their description has been omitted. Figure 3.2 shows a section of the CDF detector; Figure 3.3 shows the details of the tracking system.

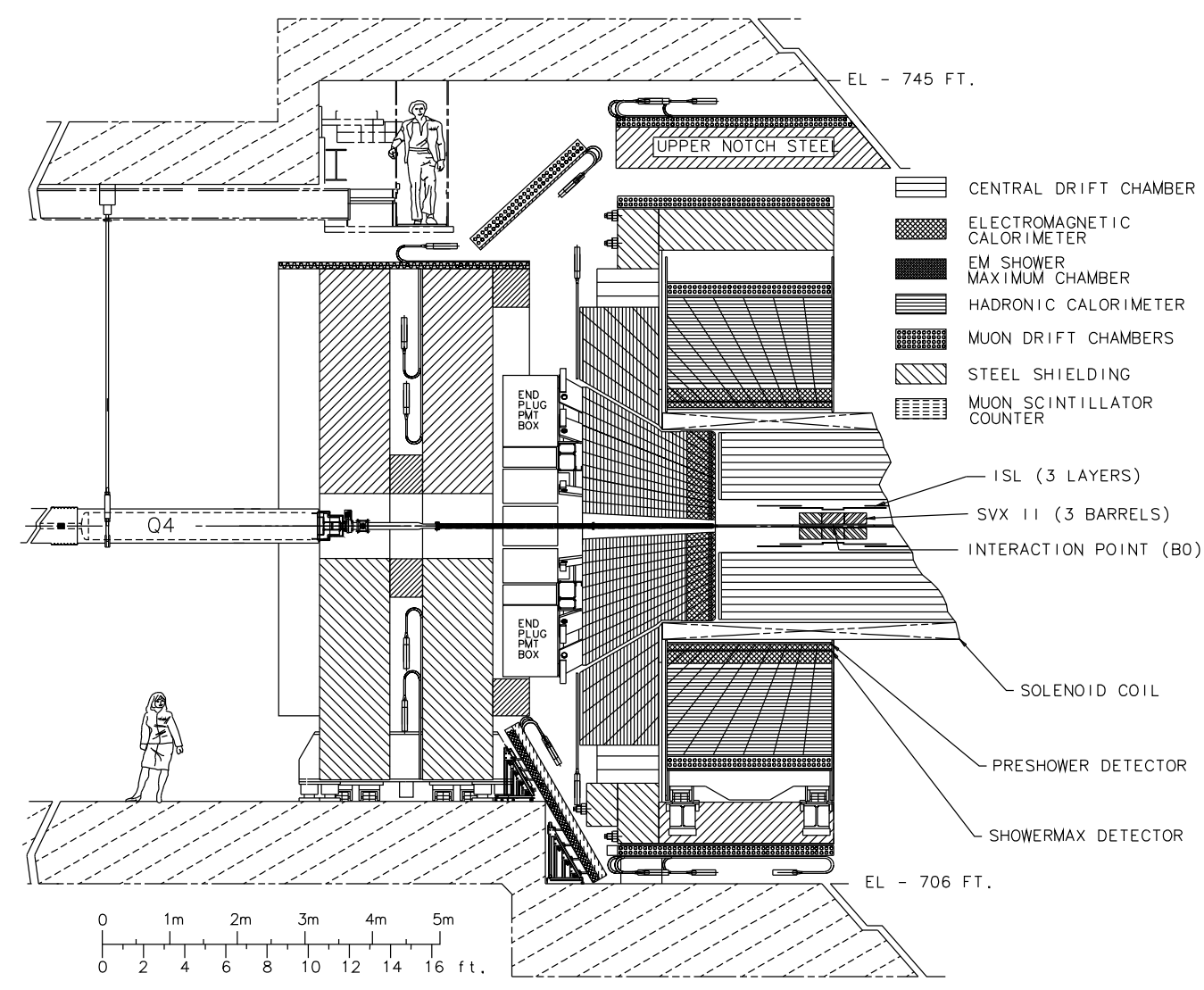

Figure 3.2: Diagram of one half of the CDF Run 2 detector. 


\section{CDF Tracking Volume}

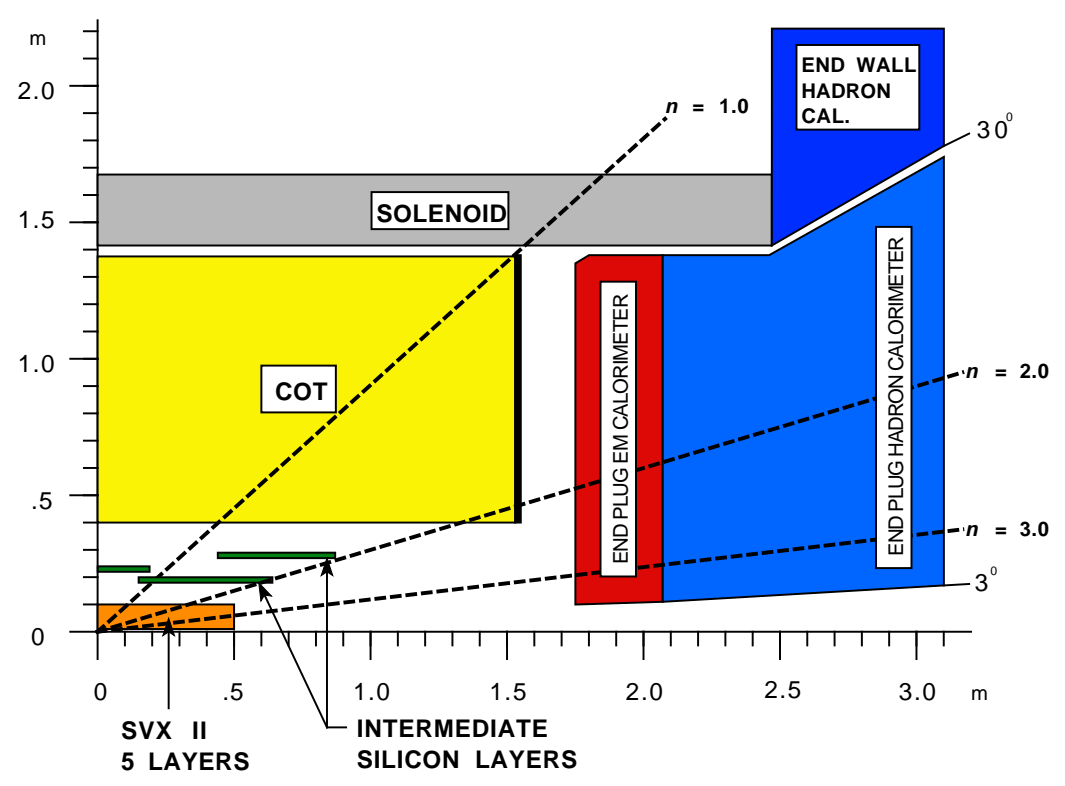

Figure 3.3: Tracking system of the CDF detector.

Many components of the detector have been upgraded for the Tevatron Run 2: the Technical Design Report [16] contains a lot of useful information about these upgrades.

\subsubsection{The CDF Coordinate System}

The CDF experiment uses a standard right-handed coordinate system:

- The $x$ axis is horizontal, pointing North (outward w.r.t. the center of the Tevatron),

- The $y$ axis is vertically upward,

- The $z$ axis is defined by the proton beam.

Spherical coordinates are also commonly used:

- The polar angle $\theta$ is defined w.r.t. the proton beam ( $+z$ direction),

- The azimuthal angle $\phi$ is defined in $x \times y$ plane w.r.t. the $x$ axis.

A commonly used variable related to the polar angle is the pseudo-rapidity $\eta=$ $-\ln (\tan (\theta / 2))$. In the limit of massless particles, the pseudo-rapidity is equivalent to the rapidity $y=(1 / 2) \ln \left(\left(E+p_{z}\right) /\left(E-p_{z}\right)\right)$, where $E$ is the energy and $p_{z}$ is the $z$ component of the momentum of the particle. The variable $r$ refers to the distance to the beam. The $(x, y)$ plane is named the transverse plane. Projected quantities onto the transverse plane are referred to as "transverse," and noted with a "T" subscript (such as transverse momentum $p_{T}$ ). 


\subsubsection{The Solenoid}

A superconducting solenoid coil creates a nearly uniform magnetic field in the tracking region $(|z|<1.5 \mathrm{~m}, r<1.4 \mathrm{~m})$. The coil itself is $4.8 \mathrm{~m}$ long and $\sim 25 \mathrm{~cm}$ thick with an inner radius $r=1.4 \mathrm{~m}$. A current of 4650 A goes through the 1164 turns of aluminum-stabilized NbTi to produce a magnetic field of 1.4 Tesla oriented in the $-z$ direction. The coil amounts to 0.85 radiation lengths at normal incidence.

\subsubsection{The Silicon Vertex Detector}

The role of a vertex detector is to provide precise tracking information near the interaction point in order to identify displaced tracks and reconstruct secondary vertices, produced by long life time particle decays. Hence it is built as close to the interaction point as possible, and must be resistant to high doses of radiation. Another requirement for this type of detector is to be able to function in a high track density environment. Semiconductor detectors satisfy such requirements.

The vertex detector $[17,18,19]$ at the CDF detector is divided into three coaxial silicon strip devices. Figure 3.4 and Figure 3.5 show the silicon vertex detector. The Layer 00 (L00) is a single-sided layer mounted directly on the beam pipe, in order to be as close as possible to the interaction point $(r \sim 1.3 \mathrm{~cm})$. The SVX II is composed of five $90 \mathrm{~cm}$ long double-sided layers $(2.55<r<10.6 \mathrm{~cm})$. The Intermediate Silicon Layer (ISL) is made of one double-sided layer in the central region $(r \sim 22.0 \mathrm{~cm})$ and two in the forward region $(r \sim 20$ and $28 \mathrm{~cm})$, and extends the tracking capability to $|\eta|=2.0$, while helping with the track reconstruction in the central region by filling the gap between the COT and the SVX II (see Figure 3.3).

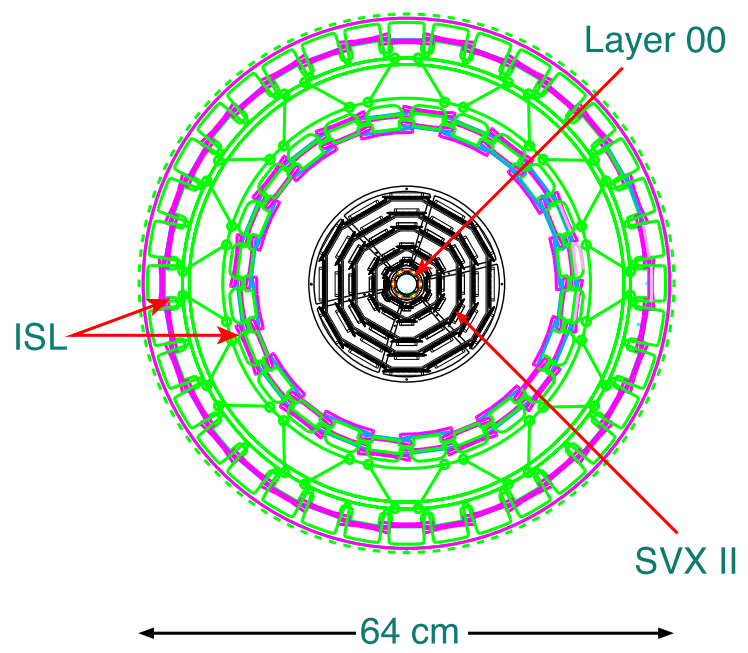

Figure 3.4: CDF silicon tracking detector in $r \times \phi$ plane. 


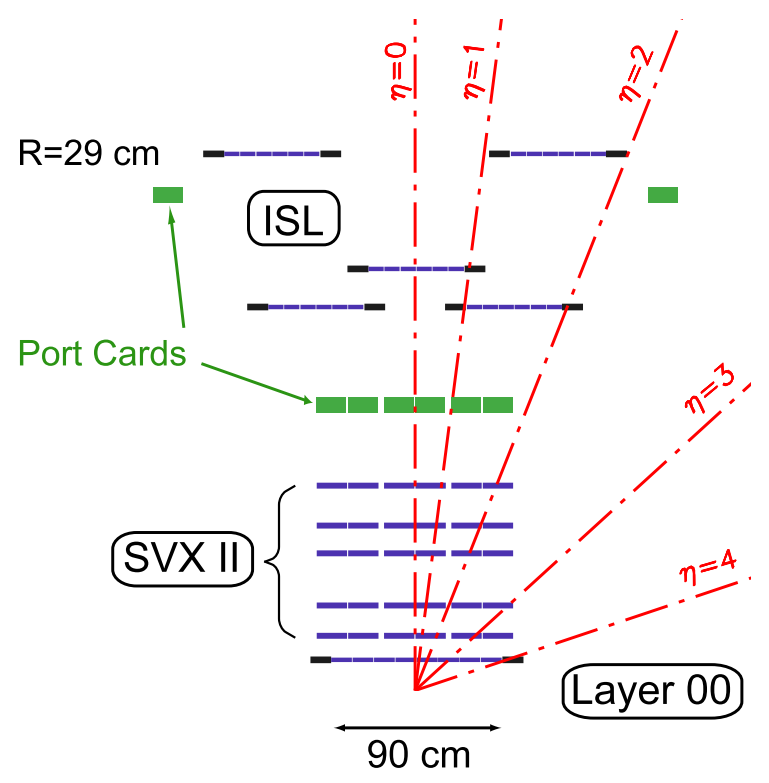

Figure 3.5: CDF silicon tracking detector in $r \times z$ plane.

\subsubsection{The Central Outer Tracker}

The Central Outer Tracker (COT) [20] is a large open-cell drift chamber built to replace the one used in Run 1 (CTC). The initially planned $132 \mathrm{~ns}$ bunch spacing for Run 2 requires a shorter drift time: the COT was designed in order to achieve a maximal drift time of $100 \mathrm{~ns}$, corresponding to a maximal drift distance of 0.88 $\mathrm{cm}$. The COT extends to $|z|<150 \mathrm{~cm}$ and radii between 40 and $137 \mathrm{~cm}$ from the beam, covering the pseudo-rapidity range $|\eta|<1.0$.

Figure 3.6 shows the layout of open cells. Each open cell contains 12 sense wires and is tilted by $35^{\circ}$ w.r.t. the radial direction. Cells are gathered into eight socalled "superlayers," as shown in Figure 3.7. There are four radial and four stereo superlayers; wires in radial layers are parallel to the $z$ axis, while in stereo layers they are tilted by $+3^{\circ}$ and $-3^{\circ}$ (alternatively) w.r.t. the $z$ axis, in order to provide measurement relative to this axis. The number of cells varies from 168 for the innermost superlayer to 480 for the outermost one. The COT amounts to $1.69 \%$ of a radiation length at normal incidence.

The gas mixture chosen is $50: 35: 15$ Argon-Ethane- $\mathrm{CF}_{4}$. Indeed, adding 15\% of $\mathrm{CF}_{4}$ reduces the aging of the wires. However, in the course of Run 2 data taking, some premature aging of the gas flux was observed, and the addition of a very small fraction of oxygen to the mixture solved the problem.

\subsubsection{The Calorimetry}

The CDF calorimetry consists of both electromagnetic calorimeters (lead absorber with fine sampling for photon and electron identification and energy measurement) and hadronic calorimeters (steel absorber for hadron energy measurement). 


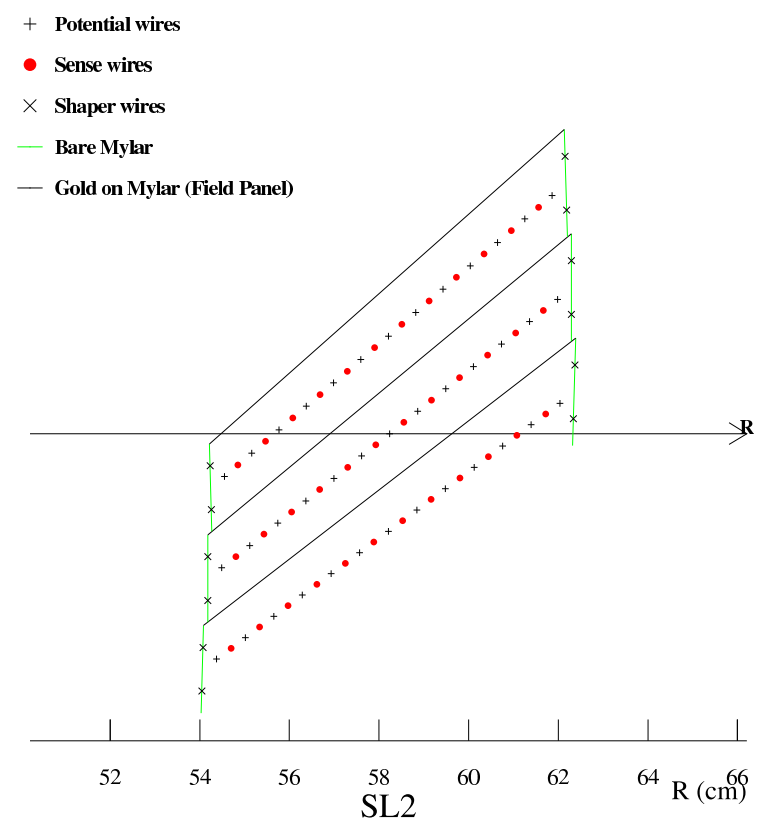

Figure 3.6: Cell layout for super layer 2.

The central region of the detector is covered by the central electromagnetic (CEM) and central hadronic (CHA) calorimeters [21, 22], in the pseudo-rapidity ranges $|\eta|<1.1$ and $|\eta|<0.9$, respectively. In the forward region, the plug electromagnetic (PEM) and hadronic (PHA) calorimeters [23, 24, 25] cover the regions $1.1<|\eta|<3.6$ and $1.3<|\eta|<3.6$, respectively. The wall hadronic (WHA) calorimeter [26] is a hadronic calorimeter that fills the gap between the CHA and the PHA in the pseudo-rapidity range $0.7<|\eta|<1.3$.

This calorimetry system provides a nearly $4 \pi$ solid angle coverage around the interaction point, which is particularly important in order to identify the presence of high- $p_{T}$ neutrinos by looking for an imbalance in the event transverse energy.

Figure 3.8 shows a wedge of the central calorimeters. The CEM is a sample device made of $5 \mathrm{~mm}$ thick layers of polystyrene scintillator, alternated with 1/8 inch thick $(3.18 \mathrm{~mm})$ layers of aluminum-clad lead. In order to maintain a constant number of radiation lengths as a function of $\theta$ (total $\sim 18 X_{0}$, and $\sim 5 X_{0}$ up to CES, see below), some lead layers are replaced by acrylic (Plexiglas), so that the actual number of absorber layers varies from 30 near the center to 20 at $|\eta| \sim 1.1$. The CEM is divided into four arches (North-West, South-West, North-East and South-East), made of identical $15^{\circ}$ modules, each of them being segmented into 10 projective towers. Thus each tower covers a solid angle of 0.1 by $15^{\circ}$ in the $\eta \times \phi$ space. The blue light emitted by the scintillators is collected on each side of the tower by acrylic wavelength shifters that convert it into green light and guide the light toward two photomultiplier tubes (PMT's, Hamamatsu R580) outside the CHA. The two most forward towers of one of the CEM and CHA modules are not instrumented (the so-called "chimney"), in order to provide access for cryogenics to the solenoid. Based on test beam data, the CEM energy resolution for an electron 


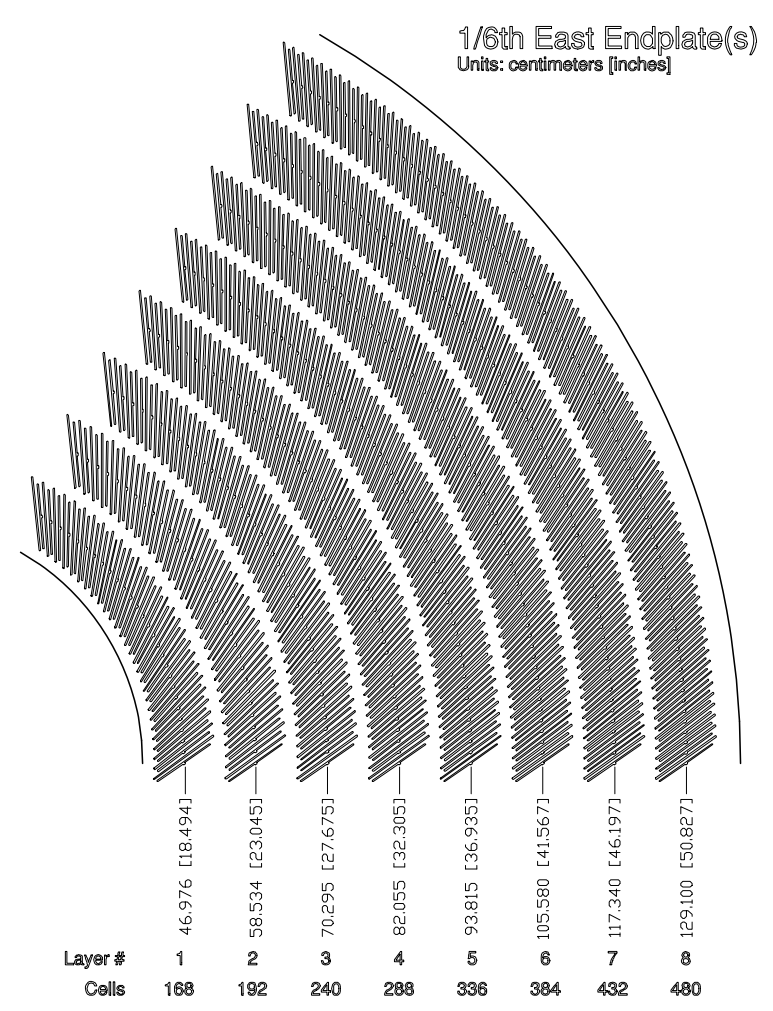

Figure 3.7: 1/6th view of COT east end plate.

going through the center of a tower is found to be

$$
\frac{\sigma_{E}}{E}=\frac{13.5 \%}{\sqrt{E(\mathrm{GeV})}} \oplus 2 \%,
$$

where the symbol $\oplus$ means that the errors are to be added in quadrature.

The central electron strip chambers (CES) are used to determine shower position and transverse shower development. They are located between the 8th lead layer and the ninth scintillator layer (counting outward), which is the expected position of shower maximum ( $~ 6 X_{0}$, including tracking and solenoid material). In each CEM module, a CES module is a multi-wire proportional chamber with 64 anode wires parallel to the beam axis, spaced $0.73 \mathrm{~cm}$ apart and split at $|z|=121 \mathrm{~cm}$, and 128 cathode strips, with a pitch of $1.67 \mathrm{~cm}$ for $|z|<121 \mathrm{~cm}$, and $2.01 \mathrm{~cm}$ for $|z|>121$ $\mathrm{cm}$. The spatial resolution achieved is $\sim 2 \mathrm{~mm}$.

The CEM is also equipped with a preshower detector (CPR), useful in discriminating between hadrons and photon/electrons. The CPR is a set of multi-wire proportional chambers with wires parallel to the beam providing transverse measurements and strip cathodes providing $z$ information, with a resolution of the order of a few millimeters.

The CHA is a sampling hadronic calorimeter surrounding the CEM, following the same segmentation $\left(0.1\right.$ by $15^{\circ}$ in $\left.\eta \times \phi\right)$. The WHA extends the CHA coverage and uses the same technology as the CHA. Altogether, a CHA + WHA wedge contains 12 


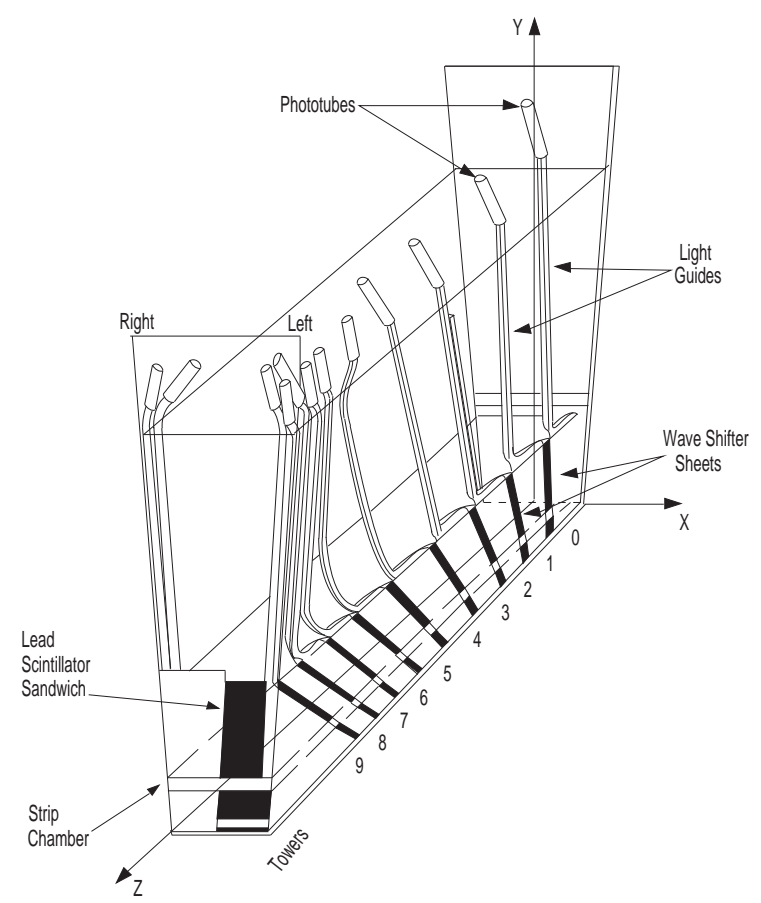

Figure 3.8: A isometric view of the central calorimeter wedge.

towers, 6 of which are fully in the CHA, 3 in the WHA, and 3 are shared between the two. The total number of interaction lengths $(\lambda)$ is approximately constant through the entire range of pseudo-rapidity, $\sim 4.5 \lambda$. The CHA is made of 32 layers of $2.5 \mathrm{~cm}$ thick steel absorber, and $1.0 \mathrm{~cm}$ thick scintillator. The WHA is made of 15 layers of $5.0 \mathrm{~cm}$ thick steel absorber, and $1.0 \mathrm{~cm}$ thick scintillator. 2 PMT's per tower are linked to the scintillators by a wavelength shifter and a light guide. The CHA and WHA single pion energy resolutions are

$$
\frac{\sigma_{E}}{E}=\frac{50 \%}{\sqrt{E(\mathrm{GeV})}} \oplus 3 \%,
$$

and

$$
\frac{\sigma_{E}}{E}=\frac{75 \%}{\sqrt{E(\mathrm{GeV})}} \oplus 4 \%
$$

respectively.

Figure 3.9 shows the plug calorimeter. The plug calorimeter was designed and built to replace the CDF Run 1 plug calorimeters and to cope with the Run 2 requirements (higher luminosity and $132 \mathrm{~ns}$ bunch spacing). It consists of an electromagnetic (PEM) and hadronic (PHA) calorimeters with the same projection segmentation. Figure 3.10 shows the segmentation pattern of a $15^{\circ}$ module: towers cover an azimuthal angle of $7.5^{\circ}$ down to $\eta=2.11$, and $15^{\circ}$ further; similarly, the segmentation in $\eta$ becomes coarser as one moves closer to the beam. Figure 3.10 also shows how towers are combined for the purpose of being used by the trigger 


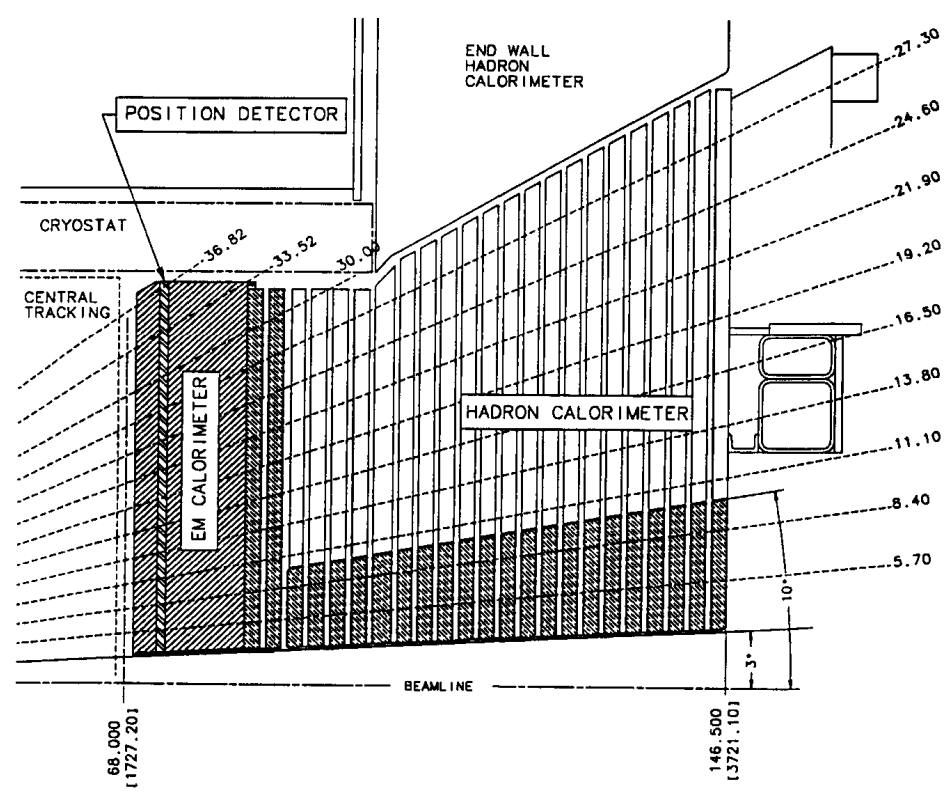

Figure 3.9: Cross section of upper part of end plug calorimeter.

system. The PEM is made of 22 layers of $4.5 \mathrm{~mm}$ lead and $4 \mathrm{~mm}$ thick scintillator tiles. Each scintillator tile is read out by a single PMT. The first layer of the PEM is a $1 \mathrm{~cm}$ thick scintillator tile read out by a multi-anode photomultiplier (MAPMT) which is used as a preshower detector. The PEM, including the preshower, amounts to a total of 21 radiation lengths. The PEM energy resolution is

$$
\frac{\sigma_{E}}{E}=\frac{16 \%}{\sqrt{E(\mathrm{GeV})}} \oplus 1 \%
$$

The plug shower maximum detector (PES) [27] is made of two sets scintillating strips that provide precise two dimensional shower position measurement (resolution $\sim 1$ $\mathrm{mm})$. The PHA is made of 23 layers of 2 inch $(5.08 \mathrm{~cm})$ thick steel absorber, and 6 $\mathrm{mm}$ thick scintillator, corresponding to 7 interaction lengths. Its resolution is

$$
\frac{\sigma_{E}}{E}=\frac{80 \%}{\sqrt{E(\mathrm{GeV})}} \oplus 5 \%
$$

Several regions of the detector have low calorimetry response (called "crack"): at the junction between East and West modules near $\eta=0$, the "chimney," near the azimuthal boundaries between wedges, and at the junction between central and plug calorimeters at $\eta \sim 1.3$. Table 3.1 summarizes some properties of the various calorimeters. 


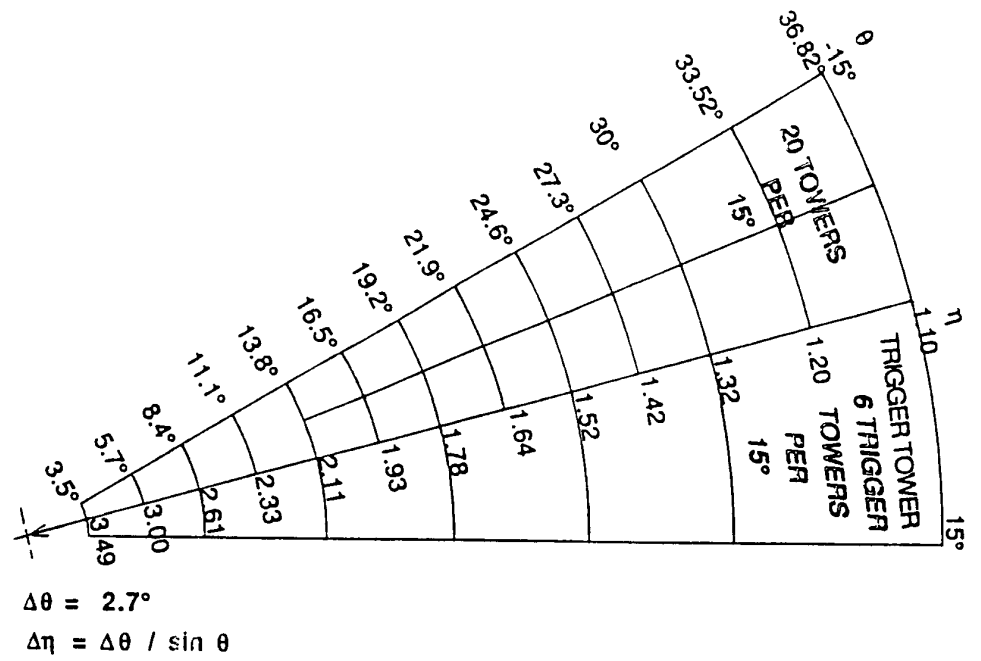

Figure 3.10: Segmentation of the plug calorimeter.

\begin{tabular}{cccccc}
\hline \hline & CEM & CHA & WHA & PEM & PHA \\
\hline$X_{0}$ & 19 & - & - & 21 & - \\
$\lambda$ & 1 & 4.7 & 4.5 & 1 & 7 \\
\# of layers & 21 & 32 & 15 & 22 & 23 \\
Absorber & $3.18 \mathrm{~mm}$ lead & $2.5 \mathrm{~cm}$ steel & $5 \mathrm{~cm}$ steel & $4.5 \mathrm{~mm}$ lead & $5.08 \mathrm{~cm}$ steel \\
Scintillator & $5 \mathrm{~mm}$ SCSN-38 & $1 \mathrm{~cm}$ PMMA & $1 \mathrm{~cm} \mathrm{PMMA}$ & $4 \mathrm{~mm}$ SCSN-38 & $6 \mathrm{~mm}$ SCSN-38 \\
$\sigma_{E} / E$ & $13.5 \% / \sqrt{E} \oplus 2 \%$ & $50 \% / \sqrt{E} \oplus 3 \%$ & $75 \% / \sqrt{E} \oplus 4 \%$ & $16 \% / \sqrt{E} \oplus 1 \%$ & $80 \% / \sqrt{E} \oplus 5 \%$ \\
\hline \hline
\end{tabular}

Table 3.1: Some properties of the CDF calorimeters. The last row shows the single particle energy resolution.

\subsubsection{The Muon System}

The muon system [28] has been upgraded for Run 2. In particular to complete the coverage in the central region, three muon detectors are used for the analysis described in this document: the Central MUon detector (CMU) and Central Muon uPgrade (CMP), covering the pseudo-rapidity range $|\eta|<0.6$, and the Central Muon eXtension (CMX), covering $0.6<|\eta|<1.0$, now have almost full $2 \pi$ azimuthal coverage. Figure 3.11 shows the coverage of each subdetector in the $\eta \times \phi$ coordinates. It should be noted that the CMU and the CMP coverages do not exactly overlap. The CMU is located outside the Central Hadronic Calorimeter $(\sim 5 \lambda)$ at a radius of $3.47 \mathrm{~m}$ from the beam. The CMP lies behind a $60 \mathrm{~cm}$ thick steel shielding (providing an extra $2.4 \lambda$ shielding at normal incidence) in order to reduce the rate of hadrons escaping the hadronic calorimeter and faking a muon. The CMX is an arch-shaped detector built around the plug calorimeter. The CMU and CMX $(\mathrm{CMP})$ can be reached by muons of $p_{T}>1.4 \mathrm{GeV} / c\left(p_{T}>2.2 \mathrm{GeV} / c\right)$. Figure 3.12 shows the number of nuclear absorption lengths a particle coming from the center 


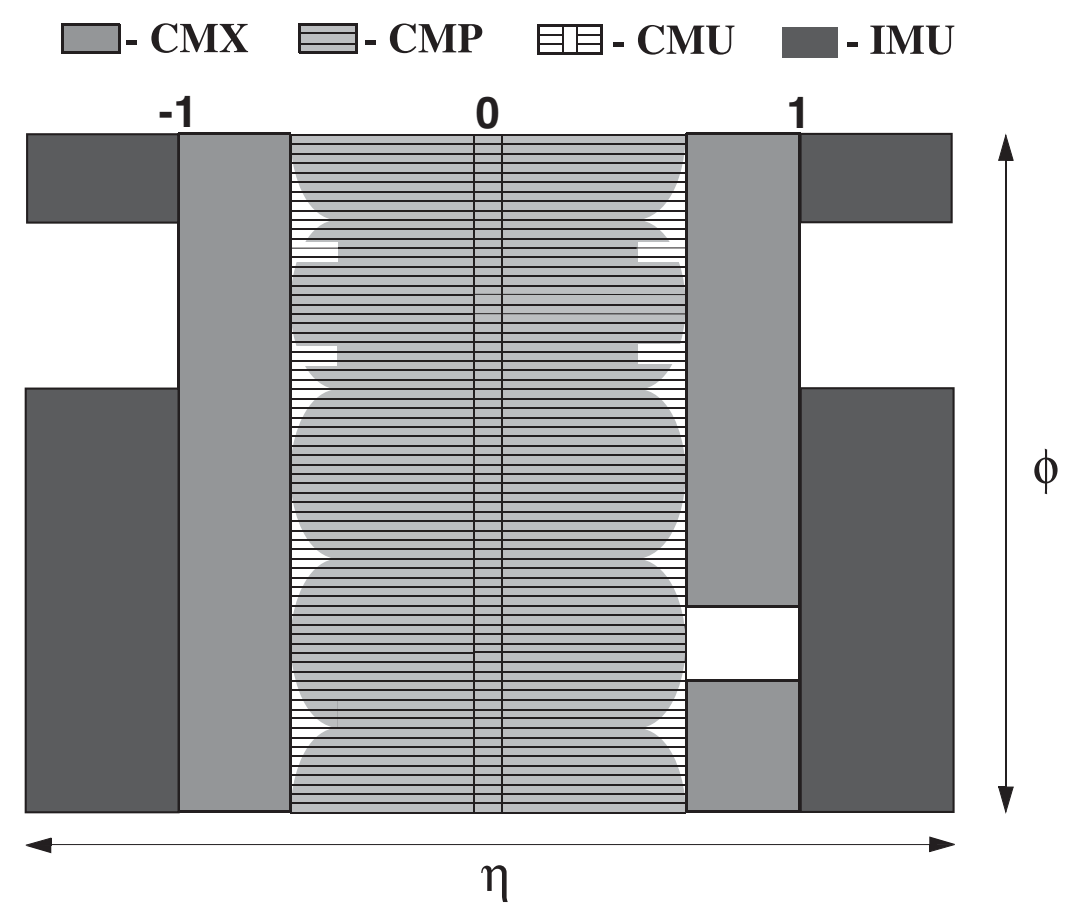

Figure 3.11: $\eta$ and $\phi$ coverage of the CDF muon system.

of the detector goes through before reaching the muon detectors, as a function of pseudo-rapidity.

The muon system relies on proportional wire chambers to provide tracking information, and scintillation counters for identifying the beam bunch. The three detectors are designed with the similar four layer configuration of drift chambers.

Figure 3.13 shows the four layer configuration of a CMU module. Each cell is $6.35 \times 2.68 \times 226 \mathrm{~cm}^{3}$ with a $50 \mu \mathrm{m}$ stainless steel sense wire in the center parallel to the beam axis. The muon $z$ location is measured using charge division at each end of the wire. The maximum drift time is $800 \mathrm{~ns}$. The wires in the first and third layers are slightly offset in $\phi$ w.r.t. the second and forth layers, in order to remove the $\phi$ ambiguity in the track reconstruction and also to provide triggering.

CMP cells are $2.5 \times 15 \times 640 \mathrm{~cm}^{3}$, providing $\phi$ information only, with a maximum drift time of $1.4 \mu \mathrm{s}$. Additionally, a layer of scintillator counters covers the outside surface of the drift chambers. Each counter covers two chambers in width, and half the chamber length, and is read out by a single photo-tube located at the center of the detector.

The CMX cells differ from the CMP cells only by their length $(180 \mathrm{~cm})$. The polar angle of the tracks is measured thanks to the stereo angle between adjacent cells, which partially overlap. Because of the conical arrangement of the cells, the overlap is more important near the inner edge, where a track can typically cross six cells. The CMP is equipped with two layers of scintillators, one on each face. Scintillator counters on the inside and outside layers are half-cell staggered, in order to effectively double the granularity of the system. Each counter is read out by a 


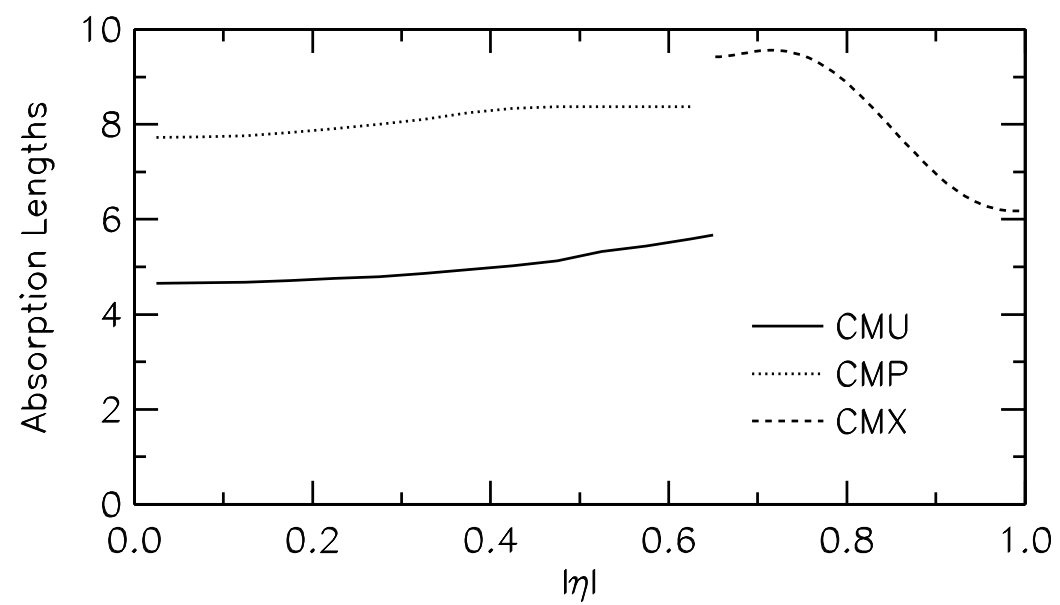

Figure 3.12: Number of nuclear absorption lengths in front of the muon systems as a function of pseudo-rapidity.

single photo-tube.

In the forward region $(1.0<|\eta|<2.0)$, the IMU detector replaces the Run 1 detector; since the IMU commissioning was still in progress during the time data was taken, it is not used for this analysis.

\subsubsection{The Čerenkov Luminosity Counter}

The Čerenkov Luminosity Counter (CLC) [29] was designed for the Tevatron Run 2 in order to achieve a precision measurement of the instantaneous luminosity up to $\sim 2 \times 10^{32} \mathrm{~cm}^{-2} \mathrm{~s}^{-1}$ and to cope with the $132 \mathrm{~ns}$ bunch spacing that was originally envisaged. The detector is made of two identical modules located near the beam pipe and inside the plug calorimeter, on each side of the interaction point. Figure 3.14 shows two views of such a module. Each module is composed of 48 conical gaseous Cerenkov counters pointing toward the interaction point and covering the pseudo-rapidity range $3.7<|\eta|<4.7$. The outer cones are $180 \mathrm{~cm}$ long, while the inner ones are $110 \mathrm{~cm}$ long; their diameter varies from 2 to $6 \mathrm{~cm}$. At the widest end of each cone (the furthest away from the interaction point), a conical mirror collects the Čerenkov light into PMT's (Hamamatsu R5800Q), capable of operating at gains up to $2 \times 10^{6}$. The modules are filled with iso-butane at atmospheric pressure; it is however possible to increase the pressure up to $2 \mathrm{~atm}$, in order to increase the yield of Čerenkov light. Iso-butane was chosen because of its large refractive index at atmospheric pressure (1.0043) and its good transparency to ultra violet light.

Because of the narrow shape and the orientation of the cones, particles produced by $p \bar{p}$ interactions close to the center of the detector are likely to go through a large portion of the CLC, producing an important light yield (several hundred photoelectrons), while particles from the beam halo or from secondary interactions transverse the detector at large angle, and have lower energy, hence producing a much smaller light signal. Thus the background is easily rejected by requiring a certain minimal 


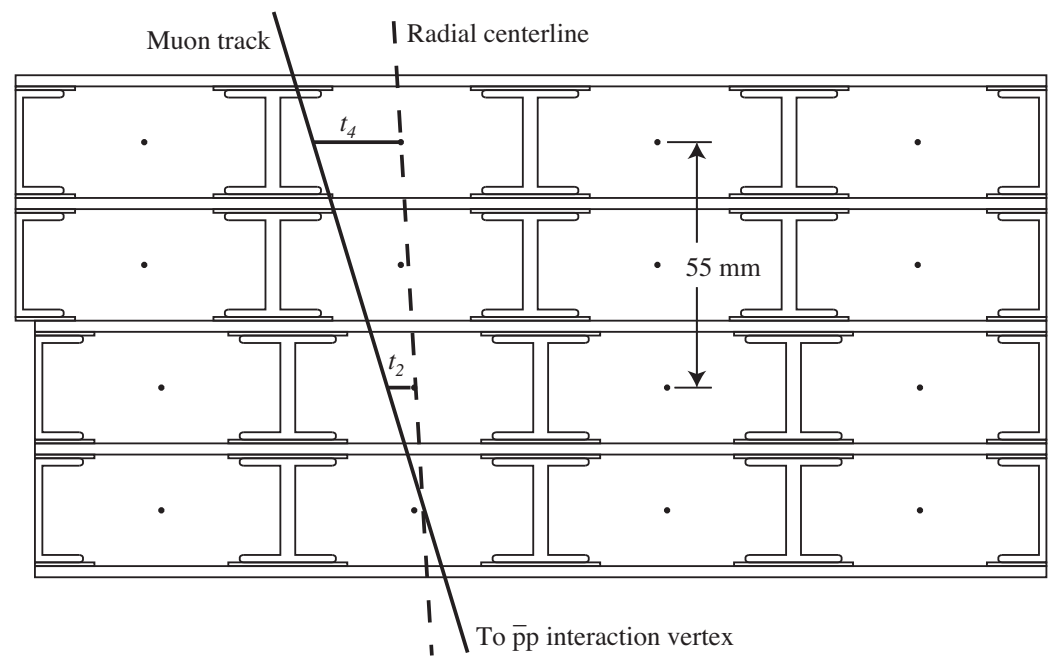

Figure 3.13: Section of a CMU module in the $x \times y$ plane.

light yield threshold in each channel; the number of particles is measured from the total light yield in the module. Thanks to the CLC excellent time resolution (less $100 \mathrm{ps}$ ), it is also possible to select hits from prompt particles by requiring time coincidence between hits in the two different modules. Time distribution of hits in each module is shown in Figure 3.15. One can clearly see the contributions from the proton beam halo, the antiproton beam halo and actual $p \bar{p}$ collisions.

The instantaneous luminosity can be expressed as a function of the measured number of hits per bunch crossing as follows:

$$
\mathcal{L}=\frac{f_{\mathrm{BC}}}{\sigma_{\text {inelastic }} \cdot \varepsilon} \cdot \frac{\left\langle N_{\text {Hits }}>\right.}{\left\langle N_{\text {Hits }}^{1}>\right.},
$$

where

- $f_{\mathrm{BC}}$ is the bunch crossing frequency,

- $\sigma_{\text {inelastic }}$ is the inelastic scattering cross section,

- $\varepsilon$ is the acceptance of the CLC for inelastic scattering events,

- $<N_{\text {Hits }}>$ is the average number of hits (particles) per bunch crossing,

- $<N_{\text {Hits }}^{1}>$ is the average number of hits (particles) for a single $p \bar{p}$ inelastic interaction.

The total inelastic scattering cross section was measured in several experiments using a luminosity independent method combining Regge theory [30] to obtain the total cross section and the optical theorem to compute the inelastic cross section from the total cross section. CDF Run 1 and E811 measurements [31] were combined, giving $\sigma_{\text {inelastic }}=60.4 \pm 2.3 \mathrm{mb}$ at $1.8 \mathrm{TeV}$, which can be extrapolated to $61.7 \pm 2.4 \mathrm{mb}$ at $1.96 \mathrm{TeV}$. This is the value used in this thesis. 


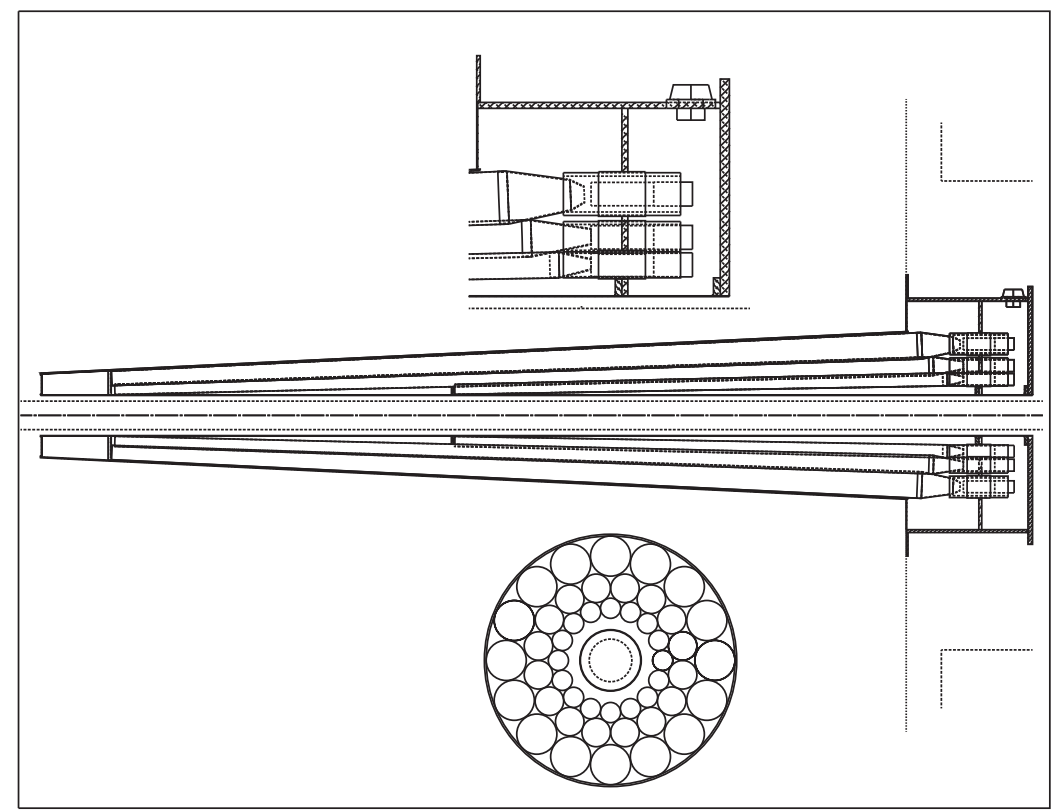

Figure 3.14: The CDF Čerenkov Luminosity Counter.

A precision of $5.9 \%$ on the luminosity is achieved with the CLC, with the main systematic uncertainties due to the detector stability (1.8\%), the CLC acceptance $(4.0 \%)$ and the inelastic cross section $(3.8 \%)$.

\subsubsection{The Trigger System}

The CDF 2 trigger system is composed of two hardware levels - Level 1 and Level 2 - and a software level - Level 3 - to select interesting events and reduce the very high rate of collisions to an acceptable one. For Run 2, the trigger system was designed to cope with a bunch crossing spacing of $132 \mathrm{~ns}(7.6 \mathrm{MHz})$; currently, the Tevatron actually operates with a 396 ns bunch spacing, which corresponds to an interaction rate of $2.5 \mathrm{MHz}$.

Figure 3.16 (Left) shows a block diagram of the data flow at CDF Run 2. In a first stage, the Level 1 gathers information from the calorimeters, the COT and the muon system and makes a decision within $5.5 \mu \mathrm{s}$. In particular, a route-based hardware tracking algorithm (the "eXtra Fast Tracker," XFT) provides tracking information from the COT to the Level 1. In order to avoid dead time while the Level 1 decision is being made, a 42 deep pipeline stores subsequent events until the Level 1 is available to process them. In a second stage, the Level 2 further selects events that pass the Level 1 selection. The Level 2 is equipped with a 4 event deep buffer, and can cope with input rates up to $50 \mathrm{kHz}$; currently, rates of the order of $25 \mathrm{kHz}$ are common. The Level 2 takes $\sim 20 \mu$ s to make a decision. The Level 2 is able to reconstruct calorimeter clusters and to use the maximum shower detector information. A novelty in hadronic physics, it is able to use the silicon vertex detector: the Silicon Vertex Trigger (SVT) uses XFT tracks as an input and tries 


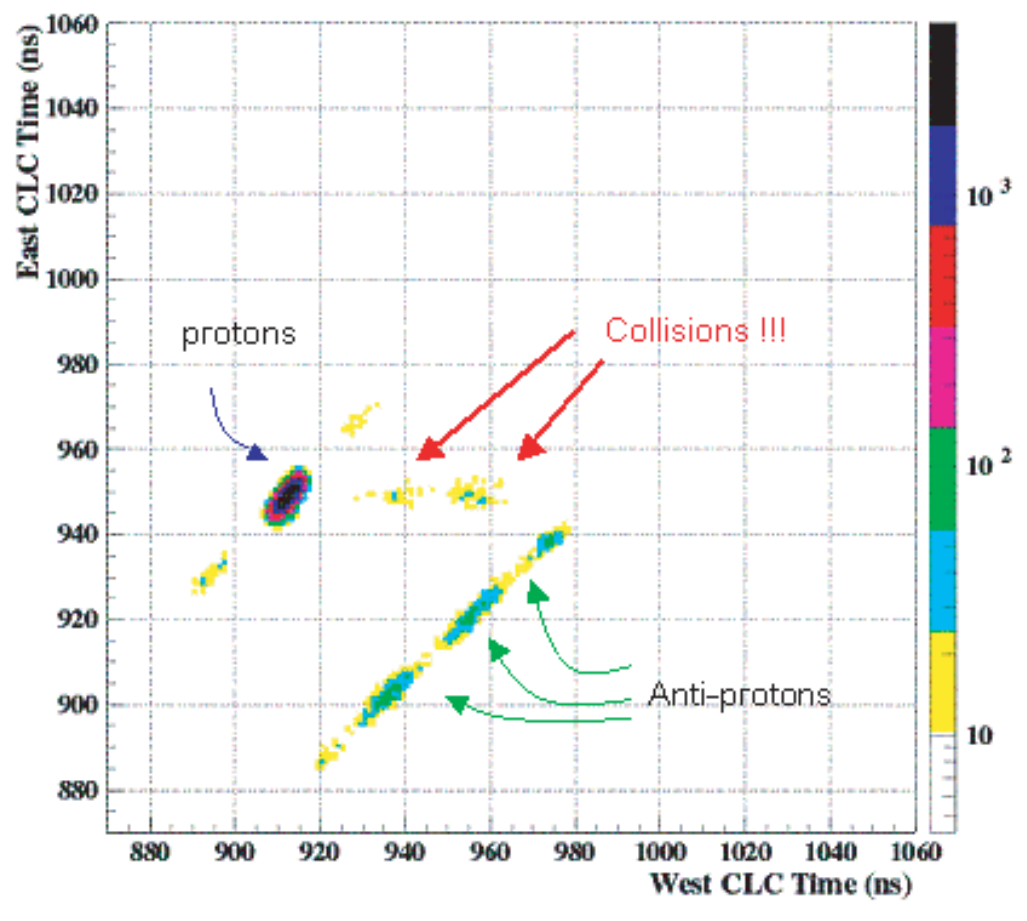

Figure 3.15: Time distribution in the East and West modules. Actual $p \bar{p}$ collisions deposit a coincident signal in the two modules.

to reconstruct tracks based on silicon hits in the neighborhood of an XFT tracks; this technique significantly reduces the number of candidate hits, hence allows very fast reconstruction, while achieving a resolution comparable with the full tracking reconstruction. The SVT is able to identify tracks that are significantly displaced from the beam location, hence selecting heavy flavor enriched events. Figure 3.16 (Right) shows what information is available to Level 1 and Level 2. Finally, a cluster of $\sim 200$ Linux PCs reconstructs the events that pass Level 2 using a simplified version of the software used for offline analysis. Level 3 reduces the rate of events from $\sim 300 \mathrm{~Hz}$ to $\sim 50 \mathrm{~Hz}$. Events that pass Level 3 are monitored in real time in the control room, and stored on tape.

\subsubsection{Detector Simulation}

Physical processes of the $p \bar{p}$ interactions are simulated by Monte Carlo (MC) generators: the PYTHIA [32], HERWIG [33, 34] and ALPGEN [35] generators are used in conjunction with the CTEQ5L parton distribution function (PDF) [36] throughout this document. The QQ v9.1 [37] decay tables are interfaced to provide proper modeling of $b$ and $c$ hadron decays. For each event, these generators produce a list of particles (and their four vector) that define the final state of the process ${ }^{1}$. In

\footnotetext{
${ }^{1}$ To be accurate, ALPGEN only produces a list of partons, while PYTHIA and HERWIG also simulate the hadronization of gluons and quarks. ALPGEN needs to be used in combination with
} 


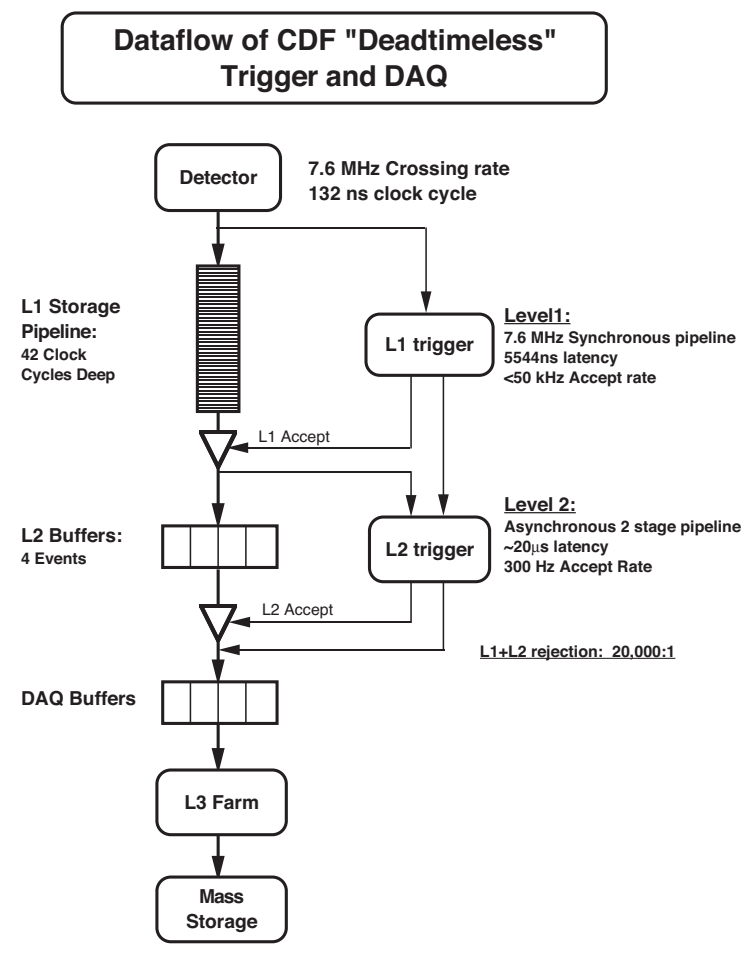

\section{RUN II TRIGGER SYSTEM}

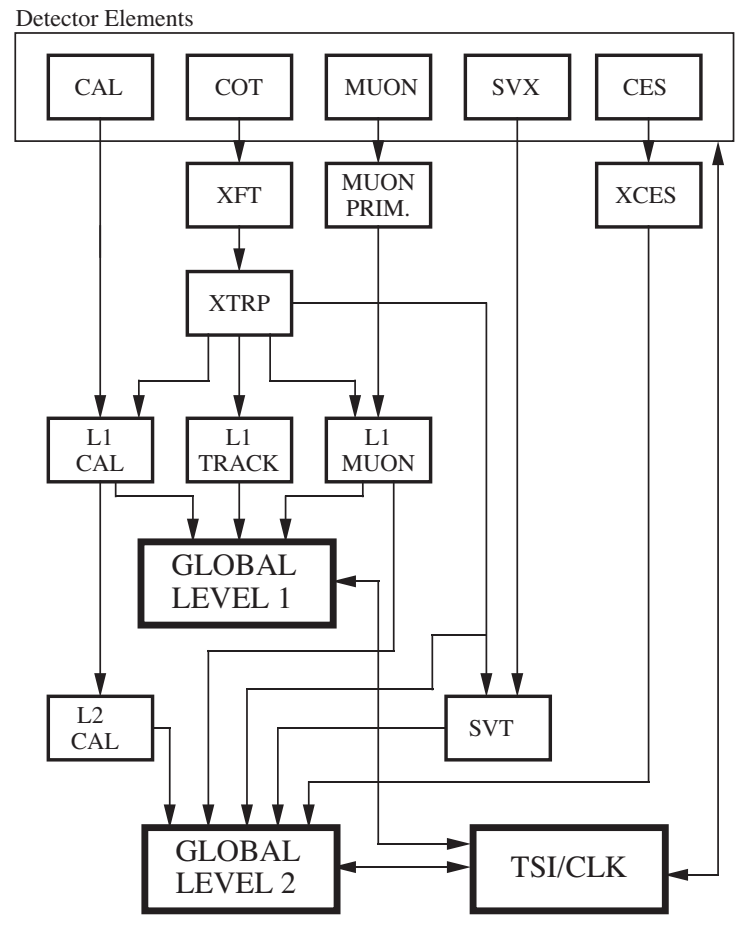

Figure 3.16: The CDF Trigger system. (Left)schematic view of DAQ and trigger system, (Right)block diagram of the Level 1 and Level 2 triggers.

order to simulate the detector response, a complex simulation of the interaction of the particles present in the final state with the detector is needed. The GEANT 3 [38] package is used together with a detailed description of the CDF sub-detectors and the passive material (such as readout system, cables, ... etc.). Specific modules are used for some of the sub-detectors. The calorimeter response is simulated with a parametrized shower simulation package GFLASH [39] tuned for single particle response and shower shape based on data. The simulation of the COT drift time uses the GARFIELD package [40]. For the silicon, a parametrized charge deposition model which is developed by CDF collaboration is used in place of GEANT 3. The simulation includes the modeling of noise and dead regions in the silicon detectors: the hardware and calibration silicon detector databases were used to account for bad silicon strips, noise and defective modules in order to reproduce the actual detector configuration in the Monte Carlo. The product of the simulation is stored in the same format as the real data, allowing the reconstruction and selection of simulated events to be performed in exactly the same way as real events.

hadronization package of another generator in order to produce the actual final state. 


\section{Chapter 4}

\section{Dataset and Event Selection}

This chapter describes the algorithms and techniques used to reconstruct charged particle trajectories, to identify electrons, muons, photons and jets, and to measure kinematic quantities. The specific event selection to the lepton + jets channel and the data samples used in this document are described in the last section.

\subsection{Tracking Reconstruction}

\subsubsection{COT Tracking}

The COT drift chamber is able to efficiently reconstruct the trajectory of charged particles ("tracks") and measure their momenta up to $|\eta|=1$. The tracking pattern recognition algorithm works in several successive steps [41]:

- Segment finding:

in each of the eight superlayers, sets of three hits consistent with a straight line trajectory are used as a segment-seed, and fit to a straight line by the method of least squares. Other hits in the superlayer lying within a road of $20 \mathrm{~ns}(\sim 1 \mathrm{~mm}$ for a drift time of $55 \mu \mathrm{m} / \mathrm{ns})$ are added to the straight line fit in an iterative procedure.

\section{- Axial tracking reconstruction:}

in the first pass of the tracking reconstruction, tracks are reconstructed in the transverse plane, using axial superlayers only. Two algorithms are run in parallel for this purpose, in order to increase the reconstruction efficiency:

- The "segment-linking" algorithm matches segments from different superlayers, then fits all the hits in the segments by minimizing a reduced $\chi^{2}$.

- The "histogram-linking" algorithm starts with a simple circle fit defined by one segment and the beam position. It then looks for hits within a 1 $\mathrm{cm}$ road about the circle and fills a $200 \mu \mathrm{m}$ binned histogram with the radius (i.e. the distance to the center of the track circle) of each hit. If the most populated bin contains at least 10 hits, a track is made out by 
those hits. Finally, the algorithm attempts to find additional hits within $750 \mu \mathrm{m}$ of the track, and refits the track.

Track duplicates (reconstructed by both algorithms) are removed in the end.

- Stereo tracking reconstruction:

information from the stereo angle superlayers is added to the axial tracks in this last step. Again, two algorithms are combined, but this time run in series:

- For every axial track, the stereo segment-linking algorithm attempts to add a stereo segment from the outer stereo superlayer, refits the track to get a first estimate of the track $z$ and $\cot \theta$, and then looks for additional stereo segments in the inner layers.

- Once the stereo segment-linking is completed, the stereo tracks are used to identify the $z$ coordinate of vertices in the event, using a histogram method. Axial tracks that could not be extended to a three dimensional track with the stereo segment-linking algorithm go through a stereo hitlinking algorithm, which scans the list of $z$ vertices and attempts to add stereo hits for a given $z$ vertex.

\section{- Refit:}

finally, tracks are refitted to take into account corrections due to the actual magnetic field map, and drift model.

The efficiency for isolated tracks of large transverse momentum $\left(p_{T}>1.5 \mathrm{GeV} / c\right)$ was found to be larger than $99 \%$. This efficiency drops to $\sim 95 \%$ for $p_{T} \sim 500$ $\mathrm{MeV} / c[42]$.

\subsubsection{Silicon Detector Tracking}

\section{Hit Clustering Algorithm}

A charged particle going through a silicon sensor often deposits charge in several contiguous strips. In the L0, the SVX and the ISL, only strips above a certain threshold and their immediate neighbors are read out. The purpose of the hit clustering algorithm is to identify sets of strips that belong to the same track, gather them into a strip cluster (or "hit"), and estimate the exact location of the track impact. In a first step, the algorithm identifies sets of contiguous strips above threshold strips ("super-clusters"). It then splits super-clusters into clusters at local minima, and identifies local maxima (peaks). If any local maximum exists, the strip at a local minimum strip charge is split between the two clusters. Finally, the hit position is estimated by the charge centroid of the cluster (weighting the central location of each strip by its charge).

\section{Outside-In Algorithm}

COT tracks are used as seeds to look for hits on the silicon detector. The hits are attached to the track in an iterative procedure, starting with the outermost layer, 
and moving inward (referred to as "outside-in" (OI) algorithm [43]). In a first pass, only axial hits are attached to the track. In a second pass, the algorithm attempts to add stereo (small angle and $90^{\circ}$ ) hits. Only tracks with at least three axial hits are kept. The algorithm looks for hits lying in a "road" four standard deviations wide around the seed track. At each iteration (i.e. in each layer), several hits may be found within the road; a new track candidate is considered for each hit found, so that a "tree" of candidate tracks is produced from a single COT seed track. In order to speed up the algorithm, only the tracks with the most and second most number of silicon hits are considered for the following iteration. The seed track is also kept for the following iteration, in case none of the hits found belong to the track. At each iteration, the track is refit in order to improve the pointing resolution and to take into account the amount of scattering material encountered. At the end of the process, only one track is kept, based on the number of silicon hits attached to it, and on the $\chi^{2}$ of the fit.

\subsection{Electron and Photon Identification}

Electron $^{1}$ and photon identification relies on the combination of the tracking and calorimeter information. Electrons and photons leave a characteristic signature in the calorimeters, since an electromagnetic (EM) shower is almost entirely contained in the EM calorimeter. Electrons are distinguished from photons in part by the slight difference shape of the EM shower, but mostly by requiring that its trajectory is reconstructed and point to the calorimeter cluster produced by the EM shower. Photons, being neutral, do not leave any trace in the tracking system.

Charged hadrons can mimic an electron signature if they shower early in the solenoid or in the EM calorimeter. Also, an electron can be misidentified as a photon if the electron track is not reconstructed. Alternatively, a photon can be misidentified as an electron if the photon converts to an electron-positron pair as it goes through material, or if a track is wrongly associated with the EM cluster. Photon conversions are identified by looking for pairs of COT tracks satisfying the following cuts:

- The two tracks have opposite sign,

- $|\Delta(x y)|<2 \mathrm{~mm}$,

- $|\Delta(\cot \theta)|<0.04$,

where $\Delta(x y)$ is the distance between the tracks in the transverse plane at the point where they are tangent in that plane, and $\Delta(\cot \theta)$ is the difference between the polar angle cotangent of the two tracks. If a candidate electron combined with a partner track is consistent with a photon conversion, it is rejected. However, if a third track can be combined with the electron partner track to form a conversion as well ("trident"), the conversion is likely to be due to a high-energy bremsstrahlung

\footnotetext{
${ }^{1}$ Electron refers to both the electron and its antiparticle, the positron.
} 
photon emitted by the initial electron as it goes through matter: in this case, the candidate electron is real, and is not rejected.

Since photon identification is not used for the analysis presented here, it is not discussed any further. Electron identification in the central calorimeter (CEM electron) for Run 2 is almost similar to the one used in Run 1 [45]. An electron candidate is considered if a tower in the CEM has $E_{T}>2 \mathrm{GeV}$ and a track points to this tower. Adjacent towers in the same wedge (i.e. towers with same $\phi$ ) are added to the cluster. The energy attributed to the electron is the total energy of the cluster. The momentum of the electron is the momentum of the track with highest- $p_{T}$ associated with the cluster; the direction of this track defines the direction of the electron, and is used to compute the electron $E_{T}=E \cdot \sin \theta$. The selection cuts shown in Table 4.1 using the variables described below efficiently select electrons and reject the background:

- The track is required to satisfy some quality requirements:

it should come from the luminous region $\left(\left|z_{0}\right|<60 \mathrm{~cm}\right)$, and have a large number of hits attached to it (at least three axial and two stereo superlayers must provide segments with at least seven hits to the track reconstruction). The track must point to an instrumented region of the detector; in particular it should be away from the tower edges and from the "chimney."

- $E$ is the total energy of the electron calorimeter cluster. $p$ is the electron momentum measured from the track. $p_{T}$ is the projection of the electron momentum onto the transverse $x \times y$ plane.

- $E_{\mathrm{HAD}}\left(E_{\mathrm{EM}}\right)$ is the energy of the electron in the hadronic (electromagnetic) calorimeter.

- $L_{\mathrm{shr}}$ is a variable relative to the EM shower shape. The energy deposited in the two towers adjacent to the tower at the center of the cluster is compared to the one expected from test beam data:

$$
L_{\mathrm{shr}}=0.14 \times \sum_{k} \frac{M_{k}-P_{k}}{\sqrt{\left(0.14 \sqrt{E_{\text {cluster }}}\right)^{2}+\sum\left(\Delta P_{k}\right)^{2}}},
$$

where the sum is over towers adjacent to the seed tower in the cluster, $M_{k}$ is a measured energy on the adjacent tower $k, P_{k}$ is the same quantity predicted using the impact point in $z$ on the strip chamber. $L_{\mathrm{shr}}$ is required to be less than 0.2 .

- The shower maximum detector (CES) is used to reject possible hadron contamination. The track is required to match a CES cluster in both axial $(|\Delta z|<3$ $\mathrm{cm})$ and azimuthal $(-1.5<Q \cdot \Delta x<3 \mathrm{~cm}$, where $Q$ is the charge of the electron) directions. In the azimuthal direction, the shower asymmetry caused by the electron bremsstrahlung is taken into account. The shape of the CES cluster is required to be similar to the one evaluated from test beam data based on a $\chi^{2}$ test $\left(\chi_{\mathrm{CES}}^{2}<10\right)$. 


\begin{tabular}{ll}
\hline \hline Variable & Value \\
\hline Fiducially & $\begin{array}{l}\text { Requirement to be in the active region of } \\
\text { the CES and CEM, and away from the } \\
\text { missing tower ("chimney") } \\
\end{array}$ \\
$\begin{array}{l}\leq 60 \mathrm{~cm} \\
\left|z_{0}\right|\end{array}$ & $\geq 3$ with $\geq 7$ hits each \\
Good COT Axial Segments & $\geq 2$ with $\geq 7$ hits each \\
Good COT Stereo Segments & $\leq 2.0$ \\
$E / p$ & $\leq 0.055+0.00045 \cdot E$ \\
$E_{\mathrm{HAD}} / E_{\mathrm{EM}}$ & $\leq 0.2$ \\
$L_{\mathrm{shr}}$ & $\leq 3 \mathrm{~cm}$ \\
$|\Delta z|$ & $\leq-1.5 \mathrm{~cm}$ and $\geq 3.0 \mathrm{~cm}$ \\
$Q \times \Delta x$ & $\leq 10$ \\
$\chi_{\mathrm{CES}}^{2} \times$ &
\end{tabular}

Table 4.1: CEM electron selection criteria.

The electron transverse energy is reconstructed from the electromagnetic cluster with the following precision [21]:

$$
\frac{\sigma_{E_{T}}}{E_{T}}=\frac{13.5 \%}{\sqrt{E_{T}(\mathrm{GeV})}} \oplus 2 \% \text {. }
$$

\subsection{Muon Identification}

Unlike electrons, muons do not initiate an EM shower in the calorimeters due to their larger mass (the muon mass, $m_{\mu}=105 \mathrm{MeV} / c^{2}$, compared to the electron mass, $\left.m_{e}=0.511 \mathrm{MeV} / c^{2}\right)$. Unlike hadrons, muons do not interact strongly, hence do not shower in the hadronic calorimeter either. As a result, muons with a transverse momentum of a few $\mathrm{GeV}$ or more deposit only a small fraction of their energy in the calorimeters due to ionization, and escape the detector. Muons are thus identified by matching hits in the muon chambers with a reconstructed track, and requiring that little energy be deposited in the calorimeter on the trajectory of the particle. In each muon system (CMU, CMP and CMX), the 4 layer structure allows to reconstruct a track segment (a so-called "stub"). A muon is reconstructed if such a stub is found in one of the muon systems and if a track points to this stub. Muons can be mimicked by hadrons that shower unusually late or not at all in the calorimeter and manage to escape the detector ("punch-through"). Another source of background is due to muons from cosmic rays, which are vetoed by using timing information of the muon chambers and of the COT. Table 4.2 shows the selection cuts that are used in order to efficiently select muons and reject backgrounds. The variables are defined as follows:

- The requirements on the COT track quality are the same as for the electron. 


\begin{tabular}{ll}
\hline \hline Variable & Value \\
\hline$\left|z_{0}\right|$ & $\leq 60 \mathrm{~cm}$ \\
$\left|d_{0}\right|$ if no Si hits & $\leq 0.2 \mathrm{~cm}$ \\
$\left|d_{0}\right|$ if Si hits & $\leq 0.02 \mathrm{~cm}$ \\
Good COT Axial Segments & $\geq 3$ with $\geq 7$ hits each \\
Good COT Stereo Segments & $\geq 2$ with $\geq 7$ hits each \\
COT exit radius (CMX-only) & $\geq 140 \mathrm{~cm}$ \\
$E_{\mathrm{EM}}$ & $\leq \max (2,2+0.0115 \times(p-100))$ \\
$E_{\mathrm{HAD}}$ & $\leq \max (6,6+0.0280 \times(p-100))$ \\
$|\Delta x|_{\mathrm{CMU}}$ & $\leq 3.0 \mathrm{~cm}$ \\
$|\Delta x|_{\mathrm{CMP}}$ & $\leq 5.0 \mathrm{~cm}$ \\
$|\Delta x|_{\mathrm{CMX}}$ & $\leq 6.0 \mathrm{~cm}$ \\
\hline \hline
\end{tabular}

Table 4.2: CMUP and CMX muon selection criteria.

- Additionally, in order to reject cosmic ray background, the track is required to have a low impact parameter $d_{0}$ (the distance between the track and the beam at the point of closest approach). If hits from the Silicon Vertex Detector are attached to the track, the requirement on the impact parameter is more stringent, since the resolution is greatly improved.

- For CMX muons (higher pseudo-rapidity), the track is required to go through all COT superlayers, i.e. exit the COT volume at a radius of $140 \mathrm{~cm}$.

- The energies $E_{\mathrm{EM}}$ and $E_{\mathrm{HAD}}$ deposited in the EM and hadronic calorimeters, respectively, along the trajectory of the muon are required to be small.

- The track is required to match the muon stub in the axial direction: $\Delta x$ is the distance in the $x \times y$ plane between the muon chambers' hits and the track extrapolated to the muon chambers' radius. The cut value varies depending on the type of muon chambers involved. There are two types of muons: CMX muons (with a stub in the CMX system), and CMUP muons (with a stub in both the CMU and CMP systems).

The muon transverse momentum is measured by the COT with the following precision:

$$
\frac{\sigma_{p_{T}}}{p_{T}}=0.0015 \cdot p_{T}(\mathrm{GeV} / c) .
$$

At high momentum, multiple scattering is negligible and the uncertainty is dominated by the drift chamber intrinsic resolution.

\subsection{Jet Reconstruction}

Jets are the experimental signature of the production of high momentum gluons and quarks, which hadronize into several collimated particles that deposit a cluster of 
energy in electromagnetic and hadronic calorimeter towers. Jets are reconstructed using a cone algorithm similar to the one used in Run 1. As a first step, every tower with transverse energy is defined as $E_{T}=E \cdot \sin \theta$, where $E$ is the energy in the tower, and $\theta$ is the polar angle of the center of the tower ${ }^{2}$. Towers with $E_{T}>100 \mathrm{MeV}$ in a cone of semi-angle $\Delta R=\sqrt{\Delta \phi^{2}+\Delta \eta^{2}}$ around the seed tower are gathered into a cluster. The centroid of these towers (in the $\eta \times \phi$ space), weighted by the energy of each tower, is computed and the list of towers in the cluster is reevaluated by now centering the cone on the energy-weighted tower centroid. In an iterative procedure, the centroid and the list of towers in the cluster are computed until the list of towers remains unchanged from one iteration to the next. The last step of the algorithm consists in dealing with overlapping jets: if the transverse energy common to two jets amounts to more than $75 \%$ of the jet with smaller transverse energy, the two jets are merged; otherwise towers in the overlapping region are assigned to the nearest jet (in the $\eta \times \phi$ space). A cone of semi-angle $\Delta R=0.4$ is used everywhere in the present document. The approximate jet energy resolution [46] for $35<E_{T}<450$ $\mathrm{GeV}$ is

$$
\sigma_{\mathrm{RMS}}=0.1 \cdot E_{T}+1.0 \mathrm{GeV}
$$

Several corrections need to be applied to the raw energy measurement in order to flatten the response of the detector and to obtain the same response in the simulations as in the data [47]: calorimeter energy scale calibration (set the calorimeter energy scale), $\eta$ dependence (makes jet energy uniform along $\eta$ ), multiple interactions (subtracts the energy coming from different $p \bar{p}$ interactions), absolute scale (corrects the jet energy measured in the calorimeter for non-linearity and energy loss in the uninstrumented regions), underlying event (subtracts the energy associated with the spectator partons in a hard collision event) and out of cone energy (adds the energy outside the clustering cone used for jet definition, taking the "jet energy" back to "parent parton energy") are taken into account.

\subsection{Missing Transverse Energy Measurement}

Even though the momentum of the initial parton is inaccessible, its momentum in the transverse plane should be null (in the detector frame). Thus, due to conservation of momentum, the final state is expected to have a null total transverse momentum as well. Because neutrinos escape from the detector without any hits, they create an apparent imbalance in the event transverse momentum. This imbalance is measured by combining the information from every calorimeter tower and defining the event missing transverse energy $\mathbb{E}_{T}$ (which is, actually, a momentum):

$$
\boldsymbol{E}_{T}=-\sum_{i=\text { towers }} \boldsymbol{E}_{T}^{i}
$$

\footnotetext{
${ }^{2}$ In order to compute tower transverse energies, the $z$ coordinate of the interaction point from which the jet is produced needs to be known; several methods, described later, can be used to evaluate the $z$ coordinate of the interaction.
} 
where $\boldsymbol{E}_{\boldsymbol{T}}^{\boldsymbol{i}}$ is a two dimensional vector (in the transverse plane) pointing from the interaction point to the center of tower $i$.

Muons deposit only a small fraction of their energy in the calorimeter: this is taken into account by adding their momentum measured from the COT to the transverse energy sum and subtracting the energy deposited in the calorimeter along the path of the muons. Finally, in order to improve the resolution on the measurement, the corrections mentioned in the previous section are applied to every jet with raw $E_{T}>8 \mathrm{GeV}$ and $|\eta|<2.4$.

\subsection{High- $p_{T}$ Lepton Sample}

The analysis presented in this document relies on data accumulated between February 2002 and August 2004, corresponding to an integrated luminosity of $319 \mathrm{pb}^{-1}$. The primary data set for this analysis is called the $W+$ jets sample, which contains most of the $W H$ events decaying into lepton + jets mode. The trigger and event selection used to isolate this sample are described below. The experimental signature of a $W H$ event in the lepton + jets decay mode is:

- The $W$ boson decaying leptonically, producing a single high- $p_{T}$ electron or muon, and large $\mathbb{E}_{T}$ due to the neutrino that escapes the detector,

- Two $b$ quark jets: two $b$ quark jets from the Higgs boson decay.

The leptonic decay of the $W$ boson gives a clear signature that is used by the hardware and software triggers. Further selections are then made offline.

\subsubsection{Triggers}

\section{High- $p_{T}$ Electron Trigger}

At the Level 1 of the trigger, calorimeter towers are gathered in pairs so that the effective $\eta \times \phi$ segmentation is $0.2 \times 15^{\circ}$. At least one trigger tower is required to have $E_{T}>8 \mathrm{GeV}$, with an $E_{\mathrm{HAD}} / E_{\mathrm{EM}}$ ratio less than 0.125 . At least one XFT track with $p_{T}>8 \mathrm{GeV} / c$ is required to point to this tower. At Level 2, a clustering algorithm combines the energy deposited in neighboring trigger towers. Towers adjacent to the seed tower found at Level 1 with $E_{T}>7.5 \mathrm{GeV}$ are added to the cluster. The total $E_{T}$ of the cluster must be larger than $16 \mathrm{GeV}$. At Level 3, a full event reconstruction and electron identification (as described in the previous sections) is performed. A three-dimensional COT track of $p_{T}>9 \mathrm{GeV}$ must point to a cluster of $E_{T}>18$ $\mathrm{GeV}$ with $E_{\mathrm{HAD}} / E_{\mathrm{EM}}<0.125$.

\section{High- $p_{T}$ Muon Triggers}

The CMUP muon hardware trigger requires hits in the CMU to match hits in the CMP. An XFT track with $p_{T}>4 \mathrm{GeV} / c$ must point to the CMU and CMP hits. Level 2 requires the presence of an XFT track with $p_{T}>8 \mathrm{GeV} / c$, not necessarily 
matching the muon hits. At Level 3, a fully reconstructed COT track with $p_{T}>$ $18 \mathrm{GeV} / c$ must match a stub in the CMU $\left(|\Delta x|_{\mathrm{CMU}}<10 \mathrm{~cm}\right)$ and in the CMP $\left(|\Delta x|_{\mathrm{CMP}}<20 \mathrm{~cm}\right)$.

The CMX muon trigger proceeds in a similar way: at Level 1, CMX hits must match the central muon extension scintillator hits and an XFT track with $p_{T}>8$ $\mathrm{GeV} / c$. There is no requirement at Level 2. At Level 3, a fully reconstructed COT track with $p_{T}>18 \mathrm{GeV} / c$ must match a stub in the $\operatorname{CMX}\left(|\Delta x|_{\mathrm{CMX}}<10 \mathrm{~cm}\right)$.

\subsubsection{Offline Selections}

The first step of the event selection isolates $W$ boson candidates by identifying events with a single high- $p_{T}$ electron or muon, where the electron has $E_{T}>20 \mathrm{GeV}$ and $p_{T}>9 \mathrm{GeV} / c$, and the muon has $p_{T}>20 \mathrm{GeV} / c$. The $W$ boson identification is completed by requiring that $\mathbb{E}_{T}>20 \mathrm{GeV}$ and that the lepton is isolated. The isolation variable $I$ is defined as the ratio of the calorimeter energy in a cone of radius $\Delta R=0.4$ around the lepton direction (but in the case of an electron, not including the electron cluster itself) to the lepton energy, and is required to satisfy $I<0.1$. The isolation requirement is meant to reject leptons from semileptonic decay of heavy flavor hadrons and leptons faked by hadrons: indeed, in both cases, the lepton candidate is produced in a jet environment and tends to be less isolated than a lepton produced by a $W$ boson decay.

The second step identifies and counts jets in the $W$ boson events. Only jets with $E_{T}>15 \mathrm{GeV}$ and $|\eta|<2.0$ are counted. With these requirements, $W H$ events are expected to give two jets. The $W+1$ jet, $W+3$ jets and $W+\geq 4$ jets samples are considered control samples, while $W$ boson events with two jets constitute the signal sample for this analysis.

In addition to the selections above, events which are flagged as $Z^{0}$ bosons and cosmic rays are removed. The $Z^{0}$ boson veto is described later in this section. In order to further reduce the top contributions, we remove events with a high- $p_{T}$ isolated track $\left(p_{T}^{\text {seed }}>20 \mathrm{GeV} / c\right)$ and charge opposite to the primary lepton. The isolated track is defined as the track with $p_{T}^{\text {seed }} /\left(p_{T}^{\text {seed }}+\Sigma p_{T}\right)>0.9$, where $p_{T}^{\text {seed }}$ is the seed track $p_{T}$ and $\Sigma p_{T}$ is the sum of track $p_{T}(>0.5 \mathrm{GeV} / c)$ in a cone of radius 0.4 around the seed track. We also require that there are no any extra jets with $E_{T}^{\text {raw }}>8 \mathrm{GeV}$ in the forward region $(2.0<|\eta|<3.0)$ or two more extra jets with $E_{T}^{\text {raw }}>8 \mathrm{GeV}$ and $E_{T}<15 \mathrm{GeV}$ in the central region $(|\eta|<2.0)$, where $E_{T}^{\text {raw }}$ is uncorrected jet $E_{T}$.

In order to purify the sample, one attempts to identify the $b$ quark jets in the $W H$ events using a secondary vertex $b$-tagging algorithm (described in Chapter 5 ). Events in which at least one of the jets is $b$-tagged are kept. The sample prior to requiring a $b$-tagged jet is referred to as the "pretag" sample.

\section{$Z^{0}$ and Dilepton Vetoes}

$Z^{0}$ boson and $t \bar{t}$ dilepton decays that contribute to the inclusive high- $p_{T}$ lepton dataset are removed by flagging the presence of a second lepton. Any event with 
two leptons satisfying the lepton identification is removed. Events where the second lepton is an electron in the plug calorimeter or a muon that fails the CMUP requirement, but has one CMU or CMP muon segment, are also removed. Finally, remaining $Z^{0}$ bosons are removed by requiring that there be no second object which forms an invariant mass with the primary lepton between 76 and $106 \mathrm{GeV} / c^{2}$. For primary muons the other object is an opposite-signed isolated track with $p_{T}>10$ $\mathrm{GeV} / c$. For primary electrons the second object may be such a track, an electromagnetic cluster, or a jet with $E_{T}>15 \mathrm{GeV}$ and $|\eta|<2.0$ that has fewer than 3 tracks in $\Delta R<0.4$ and an electromagnetic energy fraction greater than $95 \%$. 


\section{Chapter 5}

\section{Secondary Vertex $b$-Tagging: The SecVtx Algorithm}

\subsection{Introduction}

Identifying heavy flavor jets (i.e. jets containing a heavy flavor — bottom or charm - hadron) plays an important role in this analysis. While $W H$ events produce two $b$ quark jets from the hadronization of two $b$ quarks from the Higgs boson decay, most of the non- $W H$ processes found in the $W+$ jets sample do not contain heavy flavor quarks in the final state. Thus identifying $b$ quark jets significantly reduces the background.

Several methods exist. One technique looks for a low momentum electron or muon ("soft lepton tagger") coming from the semileptonic decay of heavy flavor hadron. This method is limited by the small semileptonic decay branching ratio $(\sim 11 \%)$ and by the difficulty of reconstructing low momentum leptons in a high occupancy jet environment.

Other methods take advantage of the long life time of the $B$ hadrons: with a mean life time of the order of $1.5 \mathrm{ps}(c \tau \sim 450 \mu \mathrm{m})$, together with a large relativistic boost, a $B$ hadron in a $W H$ event flies for several millimeters (on average) before decaying. The average transverse momentum of a $B$ hadron in a $W H$ is $40 \mathrm{GeV} / c$ for the Higgs boson mass of $115 \mathrm{GeV} / c^{2}$. For example, for a $40 \mathrm{GeV} / c$, neutral meson $B^{0}$ of the mass $5.28 \mathrm{GeV} / c^{2}$ corresponding to a boost $\beta \gamma=7.6$, and life time $c \tau=460$ $\mu \mathrm{m}$, the average decay length is $3.5 \mathrm{~mm}$. $B$ hadrons decay to multiple particles, producing a secondary vertex displaced from the primary interaction. Tracks coming from this secondary vertex are reconstructed by the silicon vertex detector with sufficient precision to be distinguished from the prompt tracks produced at the primary interaction.

$B$ hadron decay channels are numerous. Most of them involve neutral particles whose trajectories cannot be reconstructed, and/or a $D$ (charm) hadron, which in turn decays to several particles after a finite life time, producing a tertiary vertex making the kinematics of the decay even more complex. As a result, a full reconstruction of the $B$ hadron is impossible with a high efficiency and more inclusive methods need to be used. The SecVtx algorithm, described in Section 5.3, selects 
tracks with a large impact parameter and reconstructs a secondary vertex out of these tracks. The up side is to be relatively insensitive to the particular $B$ decay involved. The down side is that $D$ hadrons cannot be distinguished from $B$ hadrons. Although $D$ hadrons have a shorter life time and decay with lower track multiplicity than $B$ hadrons, they are tagged with a relatively high efficiency as well. Thus the SecVtx algorithm is actually a "heavy flavor tagger." In the rest of this document, the terms of "b-tagging" and "heavy flavor tagging" are used depending on the context.

This chapter consists of the following contents: Section 5.2 describes the eventby-event primary vertex algorithm. Section 5.3 describes the details of the SecVtx algorithm. In Section 5.4, the method used to define the track is discussed. The performance of the SecVtx algorithm is shown in Section 5.5. Section IV of [48] should be referred for a detailed discussion of the SecVtx algorithm.

\subsection{Event-by-Event Primary Vertex Finding}

In order to provide the initial $z$ location of the interaction, the $z$ vertex (described in Section 4.1) of the identified electron or muon is used [49]. The position of the primary vertex is then determined by fitting together the tracks within $\mathrm{a} \pm 1 \mathrm{~cm}$ window in $z$ around this vertex. The procedure starts by fitting a vertex using all tracks within the $z$ window and with impact parameter significance (relative to the average beam position) $\left|d_{0} / \sigma_{d_{0}}\right|<3$, where $\sigma_{d_{0}}$ includes the uncertainty on both the track and the beam position. The transverse profile of the beam at the $z$ of the initial vertex estimate is also used as a constraint in the fit. A pruning stage removes tracks which contribute $\chi^{2}>10$ to the fit (or the track with the largest $\chi^{2}$ contribution if the total fit reduced chi-squared per degree of freedom $\chi^{2} / n_{d}>5$, where $n_{d}$ is the degree of freedom). After the initial pruning, the fit is repeated using only the remaining tracks until a vertex with no tracks over the $\chi^{2}$ cut is found. If no tracks survive the pruning stage, then the beam profile is used for the primary vertex position estimate.

\subsection{The SecVtx Algorithm}

Secondary vertex $b$-tagging operates on a per-jet basis, where only tracks within the jet cone of radius $\Delta R=0.4$ are considered for each jet in the event. A set of cuts involving the transverse momentum, the number of silicon hits attached to the tracks, the quality of those hits and the $\chi^{2} / n_{d}$ of the track fit are applied to reject poorly reconstructed tracks. The details of this track selection are described in the next section. Clearly, only jets with at least two of these tracks can produce a displaced vertex; a jet is defined as "taggable," if the jet contains two selected tracks. Displaced tracks within the jet are selected based on the significance of their impact parameter $d_{0} / \sigma_{d_{0}}$ with respect to the primary vertex and are used as input to the SecVtx algorithm. SecVtx uses a two-pass approach to find the secondary vertices: 
- Pass 1:

The first pass requires at least three tracks with $p_{T}>0.5 \mathrm{GeV} / c$ and $\left|d_{0} / \sigma_{d_{0}}\right|>$ 2.0 , out of which it attempts to reconstruct a secondary vertex. At least one of the tracks in the fitted secondary vertex must have $p_{T}>1.0 \mathrm{GeV} / c$.

- Pass 2:

If the first pass is unsuccessful, the SecVtx algorithm performs a second pass which makes tighter track requirements $\left(p_{T}>1.0 \mathrm{GeV} / c\right.$ and $\left|d_{0} / \sigma_{d_{0}}\right|>2.5$ with one track of $p_{T}>1.5 \mathrm{GeV} / c$ ), but requires only two displaced tracks to be found in the jet, and attempts to reconstruct a two track vertex.

Once a secondary vertex is found in a jet, the two dimensional decay length $L_{x y}$ is calculated as the projection onto the jet axis, in the $r \times \phi$ plane only, of the secondary vertex vector pointing from the primary vertex to the secondary vertex. The sign of $L_{x y}$ is defined relatively to the jet direction, specifically by the angle $\alpha$ between the jet axis and the secondary vertex vector (positive for $\alpha<90^{\circ}$, negative for $\alpha>90^{\circ}$ ). Figure 5.1 shows a schematic view of a positive tag (left) and a negative tag (right). Secondary vertices corresponding to the decay of $B$

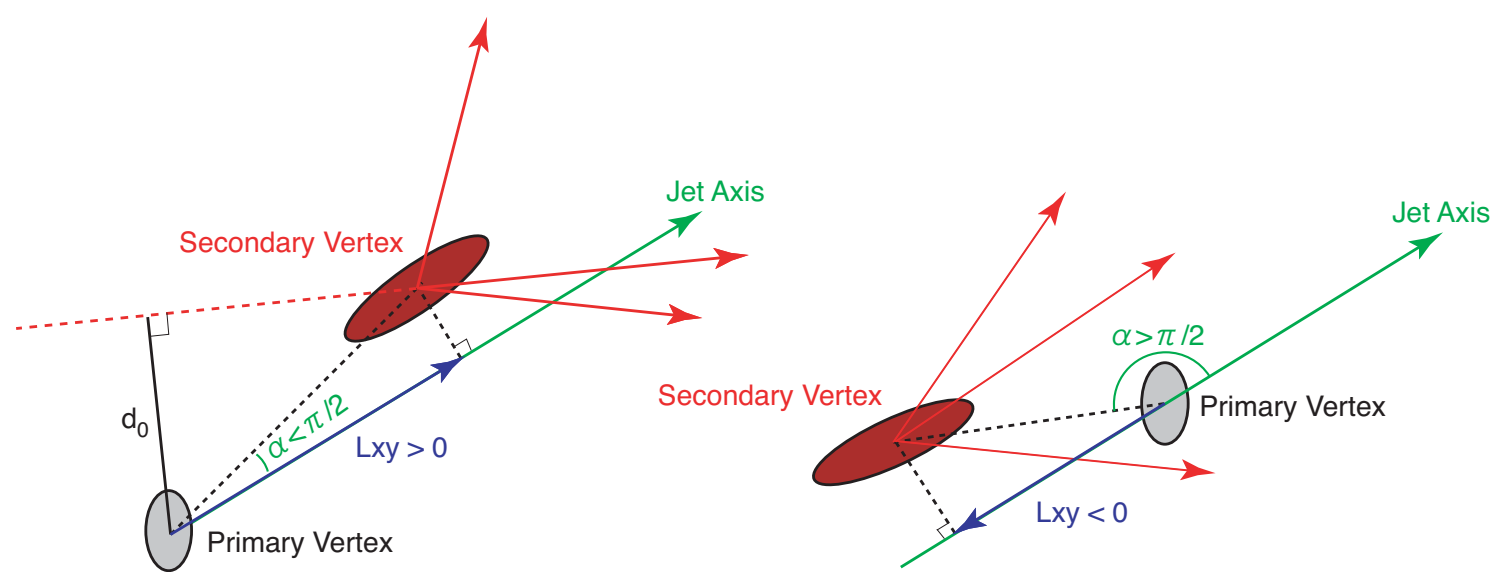

Figure 5.1: Schematic view of a positive (left) and negative (right) SecVtx tag.

and $D$ hadrons are expected to have large positive $L_{x y}$, while the secondary vertices from random mismeasured tracks are expected to be less displaced from the primary vertex and to give a symmetric $L_{x y}$ distribution w.r.t. the primary vertex. To reduce the background from the false secondary vertices, a secondary vertex is required to have $L_{x y} / \sigma_{L_{x y}}>7.5$ (positive tag) or $L_{x y} / \sigma_{L_{x y}}<-7.5$ (negative tag), where $\sigma_{L_{x y}}$ is the total estimated uncertainty on $L_{x y}$ including the errors on the primary and secondary vertices. Additionally, in order to reject secondary vertices due to material interaction [50], we converged on not allowing any vertices with exactly two Pass 1 tracks where the vertex is found between 1.2 and $2.5 \mathrm{~cm}$ with respect to the center of the $\mathrm{SVX}^{1}$. All vertices with a radius greater than $2.5 \mathrm{~cm}$ with respect to the center

\footnotetext{
${ }^{1}$ Some care must be taken since the CDF origin is taken to be the center of the COT which is not the origin of the SVX (the SVX center is shifted approximately $(x, y)=(-1 \mathrm{~mm},+1 \mathrm{~mm})$ with respect to the COT origin).
} 
of the SVX are vetoed.

The negative tags are useful for evaluating the rate of false positive tags ("mistags"), since the $L_{x y}$ distribution of fake tags is expected to be symmetric. Section 6.2 provides more details about the mistags. Figure 5.2 shows a vertex display of an actual data event.

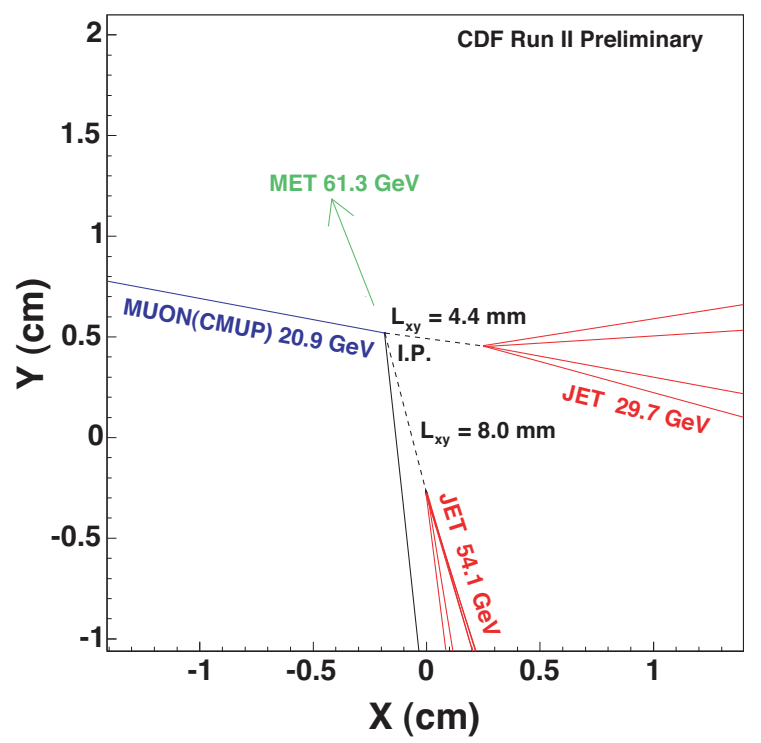

Figure 5.2: Vertex display of a $W+2$ jets candidate event with two SecVtx positive tags (run 166063, event 279746). Only tracks within a jet cone and passing the selection are shown. Prompt tracks, a muon track and tracks of the final secondary vertex fit are shown in solid black, solid blue and solid red. The $\mathbb{E}_{T}$ direction is also shown in a green arrow.

\subsection{Track Selection}

The vast majority of reconstructed tracks are prompt, meaning that they emanate from the primary interaction point whose location is estimated by the reconstructed primary vertex. Tracks that are displaced from the interaction point can be:

- misreconstructed tracks (mostly due to multiple scattering in the material),

- secondary particles produced by nuclear interactions in the detector material,

- the decay product of long life time particles ( $B$ and $D$ hadrons, but also strange particles, mostly $K_{S}^{0}$ and $\Lambda$ ).

We are interested in selecting tracks coming from the decay of a heavy flavor hadron as much as possible. Decays from $K_{S}^{0}$ and $\Lambda$ are removed explicitly by reconstructing the invariant mass of pairs of tracks. Nuclear interactions are often produced far away from the beam in the detector and at large angle: an upper bound on the impact parameter $\left|d_{0}\right|<1.5 \mathrm{~mm}$ removes the majority — but not all — of them. The track selection aims at reducing the fraction of misreconstructed tracks, the selection being described in the previous selection. Such tracks tend to have a lower 


\begin{tabular}{ll}
\hline \hline$\chi^{2}$ & $<1 ;[1,3) ;[3,6) ;[6,12),[12,25) ; \geq 25$ \\
$\#$ of hits & $\geq 2 ; 3 ; 4 ; 5$ \\
\# of good hits & $\geq 2 ; 3 ; 4$ \\
\# of missing hits & $\geq 0 ; 1 ; 2 ; 3$ \\
\hline \hline
\end{tabular}

Table 5.1: Bins used to define the track classes. For tracks with 3 hits, the hit pattern (i.e. what layers the hits are on) is also considered.

momentum, a larger fit $\chi^{2}$, fewer attached hits in the silicon detector, or hits of poorer quality than tracks coming from the decay of $B$ or $D$ hadrons. Tracks are selected based on these criteria. As a first baseline selection, only tracks satisfying the following selection are considered further:

- The track must be within the jet cone:

$$
\Delta R=\sqrt{\left(\eta_{\text {track }}-\eta_{\mathrm{jet}}\right)^{2}+\left(\phi_{\mathrm{track}}-\phi_{\mathrm{jet}}\right)^{2}}<0.4,
$$

- $p_{T}>500 \mathrm{MeV} / c$,

- $\left|d_{0}\right|<0.15 \mathrm{~cm}$,

- $\left|z_{0}-z_{\text {primary vertex }}\right|<2.0 \mathrm{~cm}$,

- Not compatible with a $K_{S}^{0}$ or $\Lambda$ decay.

In order to measure the purity of the tracks as a function of the selection cuts, a control sample made of generic QCD jet events is used. Such a sample is expected to contain few heavy flavor jets so that, to first order, one can assume that all real, well-reconstructed tracks are prompt, while misreconstructed tracks and tracks due to material effect are likely to have a large impact parameter. Thus "real tracks" (signal) are defined as tracks with an impact parameter significance $\left|d_{0} / \sigma_{d_{0}}\right|<4.0$; while fake tracks (background) are defined by $\left|d_{0} / \sigma_{d_{0}}\right|>4.0$. The purity of the track sample is then studied depending on the following variables:

- Track fit $\chi^{2}$ (normalized to the number of degrees of freedom),

- The number of SVX silicon $\phi$ (axial) hits,

- The number of missing SVX axial hits. Missing hits are identified by extrapolating the track in active modules of the silicon detector where one would expect to find a hit attached to the track, but does not find any,

- The number of good silicon hits. A good hit is required not to contain any defective strip, to be made of at most five strips, and not to be shared with any other track satisfying the baseline selection mentioned above.

A large number of classes of tracks is defined according to bins shown in Table 5.1. 


\subsection{The SecVtx $b$-Tagging Performance}

A precise understanding of the $b$-tagging algorithm performance is necessary. The CDF collaboration measures the efficiency to identify a $b$ quark jet (" $b$-tagging efficiency") using $t \bar{t} \mathrm{MC}$ sample. However, since we do not have the same $b$-tagging efficiency for MC as data yet, the ratio of the $b$-tagging efficiency $\left(\varepsilon_{\text {data }} / \varepsilon_{\mathrm{MC}}=\right.$ $0.909 \pm 0.060)$ which is estimated from $b$ semileptonic decay using jet samples is applied as the correction factor [51, 52, 53]. Figure 5.3 shows the $b$-tagging efficiencies as functions of jet $E_{T}$ and $\eta$. Although we just use the tight SecVtx tagger, the $b$-tagging efficiency for loose tagger is also shown [54]. The bands represent the systematic error on the $\varepsilon_{\text {data }} / \varepsilon_{\mathrm{MC}}$ scale factors. The decrease in efficiency at high- $E_{T}$ jet is due to worse resolution of the secondary vertex w.r.t. the jet axis.
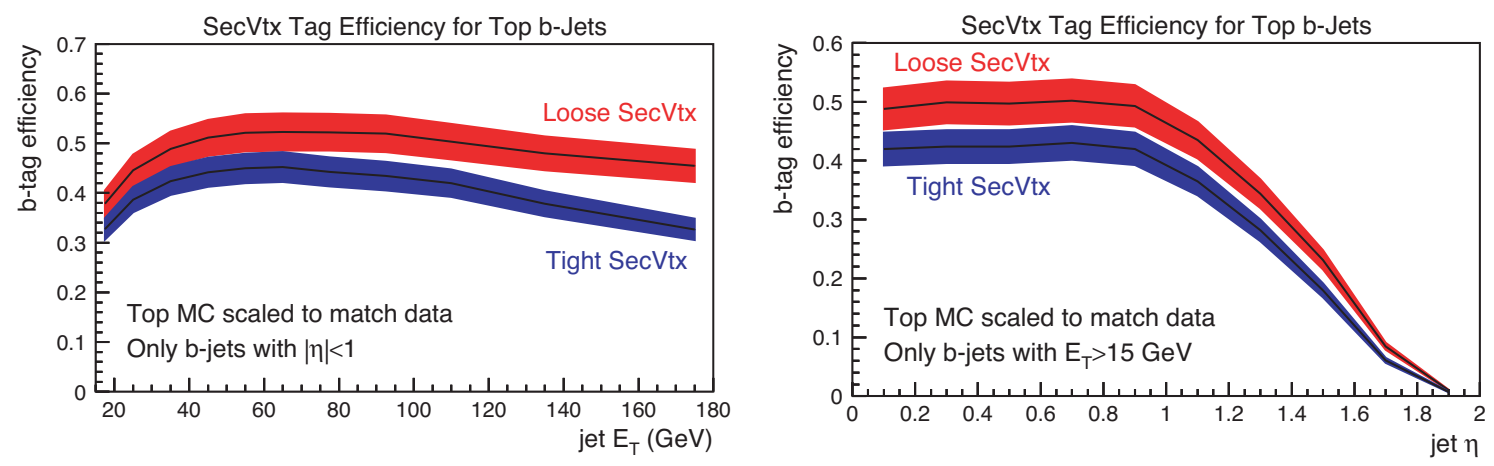

Figure 5.3: The $b$-tagging efficiency to tag $b$ quark jets in $t \bar{t} \mathrm{MC}$ samples which have been matched to $b$ quarks, using both the "tight" and "loose" tunes of the SecVtx tagger as functions of jet $E_{T}$ (left) and $\eta$ (right). The bands represent the systematic error on the $\varepsilon_{\text {data }} / \varepsilon_{\mathrm{MC}}$ scale factors.

Figure 5.4 shows the mistag rate for jets resulting from light quark/gluon fragmentation. These have been measured from inclusive jet data. The mistag rate is very low.
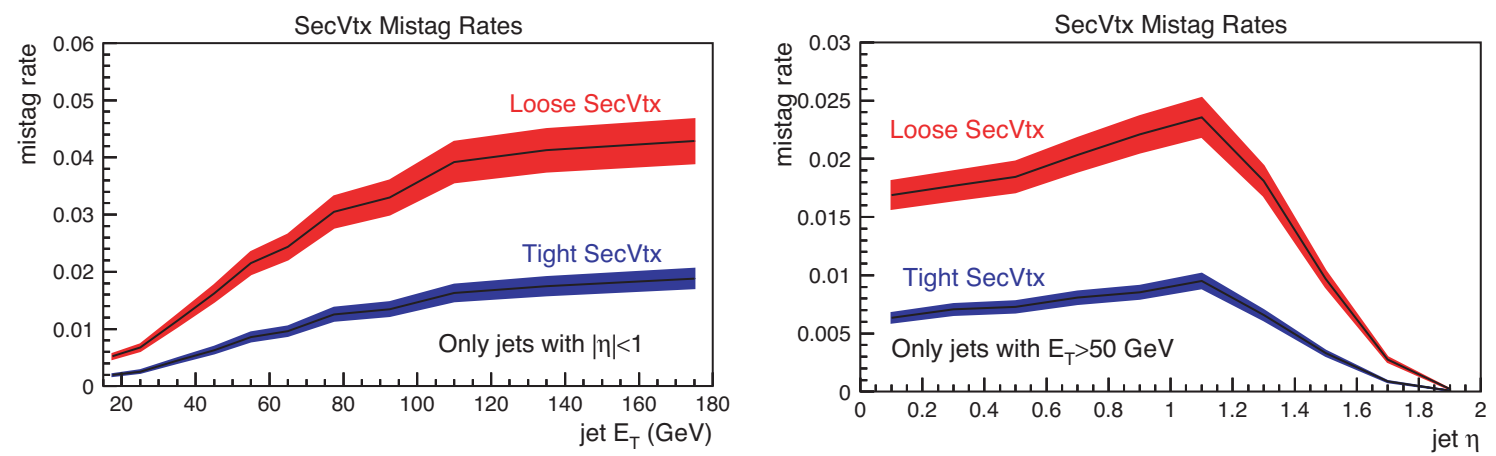

Figure 5.4: The mistag rate for jets resulting from light quark/gluon fragmentation as functions of jet $E_{T}$ (left) and $\eta$ (right). These have been measured from inclusive jet data. 


\section{Chapter 6}

\section{Background Estimation}

Several non- $W H$ processes are present in the $W+$ jets sample, even though we have optimized the event selection to isolate the $W H$ signal (Chapter 7). Here is a list of the backgrounds, whose contribution needs to be evaluated in order to search for the $W H$ production.

- $W+$ light flavor/gluon jets: the production of the $W$ boson in association with multiple light flavor/gluon jets is a background that is greatly reduced by the use of the SecVtx $b$ quark jet identification; however, events where a light flavor/gluon jet is mistagged remain as an important source of background.

- $W+$ heavy flavor jets: the production of the $W$ boson in association with heavy flavor jets is an irreducible source of background.

- non- $W$ (lepton fake): jet events faking the $W$ boson leptonic decay signature can fall into the $W+$ jets event.

- $Z^{0}+$ jets and $Z^{0}+$ heavy flavor: if one of the legs of the $Z^{0}$ boson leptonic decay is undetected, such events can fall into the $W H$ event selection. These backgrounds, though the contributions are small, are evaluated together with the $W+$ light flavor/gluon/heavy flavor jets.

- Other low rate electroweak processes with heavy flavor, such as diboson and single top productions also contribute to the background.

The estimation of each background is described in this chapter.

\subsection{Non- $W$ Background}

Jet events can fake the $W$ boson signature in several ways: the lepton can be faked by a hadron, or can come from a semileptonic decay of a heavy flavor hadron. The event with the faked $W$ boson signature is referred to as a "non- $W$." Similarly, the large $E_{T}$ can be due to a detector mismeasurement or the neutrino from the semileptonic decay. Finally, the $b$ tag can be due to an actual heavy flavor jet or a mistag ${ }^{1}$. Such

\footnotetext{
${ }^{1}$ The mistag is described in Section 6.2
} 
mismeasurements are difficult to reproduce in the simulation, since the heavy flavor content in such events is not well predicted. One must rely on the data to evaluate this source of background $[55,56]$. Since the $W / Z^{0}+$ heavy flavor backgrounds are calculated by normalizing the inclusive cross sections to the number of $W+$ jets events before $b$-tagging, it is necessary to understand the non- $W$ contamination in the pretag sample as well as in the tagged sample. More detailed discussion is found in Appendix A.

\subsubsection{Pretag Non- $W$ Background}

Generally, non- $W$ events produce non-isolated leptons and low $\mathbb{E}_{T}$, so that the regions with poor isolation (large $I$ ) and low $\mathbb{E}_{T}$ in the lepton sample are composed mostly by the non- $W$ events. These regions are used to extrapolate the expected non- $W$ contribution in the signal region (good isolation ( The lepton data sample is divided into the following four regions:

- Region A: $I>0.2$ and $\not_{T}<15 \mathrm{GeV}$,

- Region B: $I<0.1$ and $\not_{T}<15 \mathrm{GeV}$,

- Region C: $I>0.2$ and $\not_{T}>20 \mathrm{GeV}$,

- Region D: $I<0.1$ and $\mathbb{E}_{T}>20 \mathrm{GeV}$,

where Region $\mathrm{D}$ is the $W$ boson signal region. The main assumption of the method is to consider that isolation and $\mathbb{E}_{T}$ are uncorrelated for non- $W$ events, so that the ratio of non- $W$ events with $I>0.2$ and $I<0.1$ in the low $\mathbb{E}_{T}$ region is the same as in the high $\mathbb{E}_{T}$ region. Then the number of non- $W$ events in the signal region $\mathrm{D}$ is given by

$$
N_{\mathrm{D}}^{\text {non }-W}=\frac{N_{\mathrm{B}} \times N_{\mathrm{C}}}{N_{\mathrm{A}}},
$$

where $N_{\mathrm{X}}$ denotes the number of events in Region $\mathrm{X}$. The non- $W$ background is calculated separately for the electron and muon channels and for each jet multiplicity.

\subsubsection{Tagged Non- $W$ Background}

Two methods are employed and combined to estimate the number of non- $W$ events in the $b$-tagged " $W+$ jets" sample.

One method evaluates the $b$-tagging rate of non- $W$ events in the signal sideband region, and applies this rate to the pretag non- $W$ estimate in the signal region D. Although the heavy flavor content of the non- $W$ events is not well known (in particular, the presence of a real lepton enhances the heavy flavor content in non- $W$ events), the $b$-tagging rate in Region $\mathrm{B}$ is used as an estimate of the $b$-tagging rate in the signal region D (Region $B$ rate method). In order to reduce the statistical uncertainty, the $b$-tagging rate per taggable jet is measured and it is applied to the number of taggable jets in the signal region $\mathrm{D}$. 
Another method (tagged method) regards the isolation distribution of leptons in the tagged events in the low $\mathbb{E}_{T}$ region as an unbiased isolation distribution of the non- $W$ events. This isolation distribution can then be used to estimate the number of non- $W$ events in high $\mathbb{E}_{T}$. With the limited statistics in the present data sample, this method is applied in the two isolation bins $(I<0.1$ and $I>0.1)$, effectively applying Equation 6.1 to the tagged event sample, where the upper isolation cut is lowered from 0.2 to 0.1 in order to gain statistics in the control regions. For the same reason, the $N_{\mathrm{B}} / N_{\mathrm{A}}$ ratios are also measured for two jet bins ( $W+1$ jet and $W+\geq 2$ jets samples).

\subsubsection{Systematic Uncertainty}

In order to validate the method and estimate the systematic uncertainty associated with the background estimation, we divide $\mathbb{E}_{T}$ and isolation into the following sideband regions:

- Region A : $I>0.2$ and $\mathbb{E}_{T}<15 \mathrm{GeV}$,

- Region E : $0.1<I<0.2$ and $\not_{T}<15 \mathrm{GeV}$,

- Region $\mathrm{C}: I>0.2$ and $\mathbb{E}_{T}>20 \mathrm{GeV}$,

- Region F : $0.1<I<0.2$ and $\mathscr{E}_{T}>20 \mathrm{GeV}$,

- Region A": $I>0.1$ and $\mathbb{E}_{T}<10 \mathrm{GeV}$,

- Region $\mathrm{A}^{\prime}: I>0.1$ and $10<\mathbb{E}_{T}<20 \mathrm{GeV}$,

- Region B": $I<0.1$ and $\mathbb{E}_{T}<10 \mathrm{GeV}$,

- Region B' $: I<0.1$ and $10<\mathbb{E}_{T}<20 \mathrm{GeV}$.

We calculate the ratios, $G=\left(N_{\mathrm{E}} \cdot N_{\mathrm{C}}\right) /\left(N_{\mathrm{A}} \cdot N_{\mathrm{F}}\right)$ and $G^{\prime}=\left(N_{\mathrm{B}}, \cdot N_{\mathrm{A}},\right) /\left(N_{\mathrm{A}}, \cdot N_{\mathrm{B}}{ }^{\prime}\right)$ for both pretag and $b$-tagged samples, shown in Table 6.1. Here Region $\mathrm{F}$ is the isolation sideband and $\left(N_{\mathrm{E}} \cdot N_{\mathrm{C}}\right) / N_{\mathrm{A}}$ is the estimation in Region $\mathrm{F}$ as described in Section 6.1.1. Region B' is the $\mathbb{F}_{T}$ sideband and $\left(N_{\mathrm{B}}, \cdot N_{\mathrm{A}},\right) / N_{\mathrm{A}}$ " is also the estimation in this region. If the extrapolations from isolation or $\mathbb{E}_{T}$ sideband regions are valid, then the fractions $G$ and $G^{\prime}$ should be equal to 1 . Any deviation from unity will be assigned as a part of systematic uncertainty. For both pretag and tagged samples, the methods seem to valid at a $25 \%$ level.

\subsubsection{Summary of Non- $W$ Background}

Table 6.2 and Table 6.3 show the estimated number of $b$-tagged non- $W$ events from the Region B rate method and the tagged method. A systematic uncertainty of $25 \%$ is attached. Both background estimations are weight averaged, as shown in Table 6.4 . 


\begin{tabular}{|c|c|c|c|c|}
\hline & \multicolumn{2}{|c|}{ Pretag } & \multicolumn{2}{|c|}{ Tagged } \\
\hline & Electron & Muon & Electron & Muon \\
\hline \multicolumn{5}{|c|}{$G$ :Region $\mathrm{F}\left(0.1<I<0.2\right.$ and $\left.\mathscr{E}_{T}>20 \mathrm{GeV}\right)$} \\
\hline$W+1 \mathrm{jet}$ & $1.00 \pm 0.03$ & $0.63 \pm 0.03$ & $0.9 \pm 0.1$ & $0.7 \pm 0.2$ \\
\hline$W+2$ jets & $0.84 \pm 0.05$ & $0.7 \pm 0.1$ & $1.0 \pm 0.2$ & $0.8 \pm 0.3$ \\
\hline \multicolumn{5}{|c|}{ 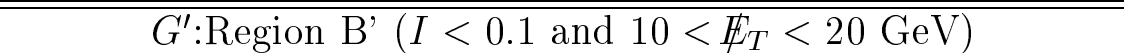 } \\
\hline$W+1$ jet & $1.05 \pm 0.01$ & $0.76 \pm 0.02$ & $1.05 \pm 0.08$ & $0.82 \pm 0.12$ \\
\hline$W+2 \mathrm{jets}$ & $0.77 \pm 0.02$ & $0.65 \pm 0.04$ & $0.79 \pm 0.12$ & $0.72 \pm 0.20$ \\
\hline
\end{tabular}

Table 6.1: The ratio of extrapolated and observed non- $W$ backgrounds in Regions $\mathrm{F}$ and B' for pretag and $b$-tagged event samples.

\begin{tabular}{ccccc}
\hline \hline Jet Multiplicity & 1jet & 2jets & 3jets & $\geq$ 4jets \\
\hline electron channel & $23.7 \pm 6.0$ & $10.6 \pm 2.8$ & $2.7 \pm 0.8$ & $1.0 \pm 0.3$ \\
muon channel & $6.6 \pm 1.3$ & $3.8 \pm 0.9$ & $1.6 \pm 0.4$ & $0.7 \pm 0.2$ \\
\hline \hline
\end{tabular}

Table 6.2: The estimated number of $b$-tagged non- $W$ events (Region B rate method).

\begin{tabular}{ccccc}
\hline \hline Jet Multiplicity & 1jet & 2jets & 3jets & $\geq 4$ jets \\
\hline electron channel & $37.4 \pm 9.9$ & $17.7 \pm 5.0$ & $4.2 \pm 1.5$ & $1.7 \pm 0.7$ \\
muon channel & $5.5 \pm 1.3$ & $3.4 \pm 1.1$ & $2.3 \pm 0.7$ & $1.2 \pm 0.5$ \\
\hline \hline
\end{tabular}

Table 6.3: The estimated number of $b$-tagged non- $W$ events (tagged method).

\begin{tabular}{ccccc}
\hline \hline Jet Multiplicity & 1jet & 2jets & 3jets & $\geq$ 4jets \\
\hline electron channel & $27.3 \pm 5.1$ & $12.3 \pm 2.5$ & $3.0 \pm 0.7$ & $1.1 \pm 0.3$ \\
muon channel & $6.0 \pm 1.2$ & $3.8 \pm 0.8$ & $1.8 \pm 0.4$ & $0.8 \pm 0.2$ \\
\hline \hline
\end{tabular}

Table 6.4: The estimated number of $b$-tagged non- $W$ events (average). 


\begin{tabular}{ccccc}
\hline \hline Jet Multiplicity & 1jet & 2jets & 3jets & $\geq$ 4jets \\
\hline Mistags & $98.0 \pm 7.9$ & $39.3 \pm 3.1$ & $12.1 \pm 1.2$ & $5.9 \pm 0.8$ \\
\hline \hline
\end{tabular}

Table 6.5: The estimated number of mistag background contribution.

\subsection{W+Light Flavor/Gluon Jets (Mistags)}

A light flavor/gluon jet that ends up being positively $b$-tagged by SecVtx is referred to as a "mistag." Mistag background events are $W+$ jets events where the tagged jet does not result from the decay of a heavy flavor hadron. Mistags are caused mostly by random overlap of tracks which are displaced from the primary vertex due to tracking errors. There are some contributions from $K_{S}^{0}$ and $\Lambda^{0}$ decays and nuclear interactions with the detector material (the beam pipe or the inner silicon layers). Since the MC fails in describing them properly, these effects are measured directly from jet data samples without relying on the detector simulation.

Because the SecVtx algorithm is symmetric in its treatment of $d_{0}$ and $L_{x y}$ significance, the mistags should occur at the same rate for $L_{x y}>0$ and $L_{x y}<0$. Therefore, a good estimate of the positive mistag rate due to resolution effect can be obtained from the negative tag rate [57]. The samples of jet triggers are used to measure the rate of negative tags for taggable jets. The mistag rate is parametrized as a function of four jet variables $-E_{T}$, track multiplicity, $\eta$ and $\phi-$ and one event variable $\sum E_{T}$, the scalar summed $E_{T}$ of all jets with $E_{T}>10 \mathrm{GeV}$ and $|\eta|<2.4$. These parametrized rates are used to obtain the probability that a given jet will be negatively tagged.

In estimating the mistag background, each jet in the pretag sample is weighted with its mistag rate. The sum of the weights over all jets in the sample is then scaled down to account for the fraction of pretag events which are due to non- $W$ background. The low mistag rate per jet means that negligible number of events has multiple mistagged jets; the number of mistagged jets is well approximated to the number of events with (at least) one mistagged jet. This method is tested to predict the negative SecVtx tagged events in the lepton + jets sample. As compared in Figure 6.1 as a function of the jet multiplicity, there is a fair agreement in the shape and the normalization between the prediction and observation.

For the estimation of the number of fake positive tags, a correction factor of $1.27 \pm 0.13$ is applied to account for additional mistags of light flavor/gluon jets due to material interactions and long lived light quark hadrons [50]. The results for the mistag estimate are shown in Table 6.5. The error includes statistical uncertainties from the pretag sample, and takes into account the small effect of correlation between mistag weights that come from the same bin in the mistag matrix. In addition, there is a $8 \%$ systematic uncertainty accounting for the sample dependence of the mistag rate parametrization [52]. 


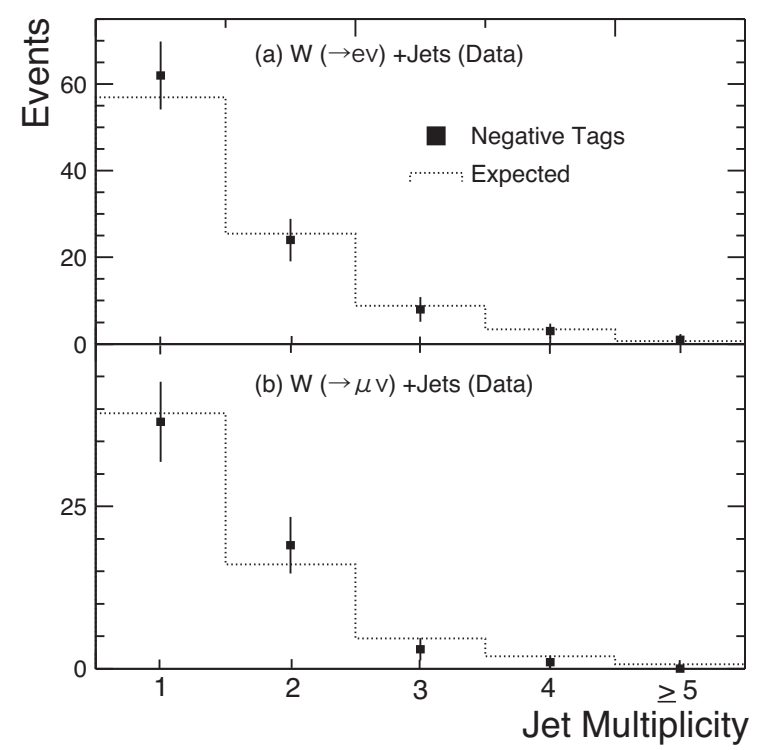

Figure 6.1: Comparison of observed and predicted negative SecVtx tags as a function of jet multiplicity in the lepton + jets data sample. (a)electron channel, (b)muon channel.

\section{3 $W+$ Heavy Flavor Jets $(W+b \bar{b}, W+c \bar{c}$ and $W+c)$}

Heavy flavor production in association with a vector boson (e.g. $W+b \bar{b}, W+$ $c \bar{c}, W+c)$ contributes significantly to the $W H$ background in the $b$-tagged $W+$ jets sample, even though $W+$ light flavor/gluon jets production dominates in the pretag sample. Several MC generators are capable of performing matrix element calculations for $W / Z^{0}+$ jets production, even to high jet multiplicity, but these generators use leading-order calculations. As a result, the overall normalization of these contributions has a large theoretical uncertainty. However, the relative contributions of the various diagrams are rather well-defined. Thus a matrix element MC program is used to estimate the relative fraction of $W+$ heavy flavor production, but the overall normalization of the $W+$ jets production is measured from the data. Combining the two results gives an estimate of the $W+$ heavy flavor signal.

The new event generator ALPGEN [35] is used in this study. The ALPGEN calculates exact matrix elements at leading order for a large set of parton level processes in QCD and electroweak interactions, taking into account all heavy quark masses, spins and color flows. Table 6.6 shows the heavy flavor fractions evaluated using the ALPGEn [59]. The numbers of $W+b \bar{b}, W+c \bar{c}$ and $W+c$ events are given by multiplying the heavy flavor fractions by the pretag event count, after subtracting the QCD background. Estimates of the $b$-tagged background are then obtained by multiplying the SecVtx tagging efficiencies summarized in Table 6.6. The SecVtx tagging efficiencies include the scale factor $\left(\varepsilon_{\text {data }} / \varepsilon_{\mathrm{MC}}=0.909 \pm 0.060\right)$, which corrects the $b$-tagging efficiency differences between data and $\mathrm{MC}[51,52,53]$. 


\begin{tabular}{|c|c|c|c|c|}
\hline Jet Multiplicity & 1jet & 2 jets & 3jets & $\geq 4$ jets \\
\hline \multicolumn{5}{|c|}{ 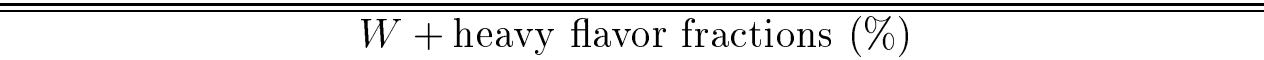 } \\
\hline $\bar{c} W+b b(1 b)$ & 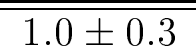 & 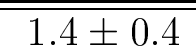 & $2.0 \pm 0.5$ & $2.2 \pm 0.6$ \\
\hline$W+b \bar{b}(2 b)$ & & $1.4 \pm 0.4$ & $2.0 \pm 0.5$ & $2.6 \pm 0.7$ \\
\hline$W+c \bar{c}(1 c)$ & $1.6 \pm 0.4$ & $2.4 \pm 0.6$ & $3.4 \pm 0.9$ & $3.6 \pm 1.0$ \\
\hline$W+c \bar{c}(2 c)$ & & $1.8 \pm 0.5$ & $2.7 \pm 0.7$ & $3.7 \pm 1.0$ \\
\hline$W+c$ & $4.3 \pm 0.9$ & $6.0 \pm 1.3$ & $6.3 \pm 1.3$ & $6.1 \pm 1.3$ \\
\hline \multicolumn{5}{|c|}{ SecVtx $b$-tagging efficiencies (\%) } \\
\hline $\bar{~} W+b b(1 b, \geq 1 \mathrm{tag})$ & $26.8 \pm 2.0$ & $27.8 \pm 2.2$ & $29.3 \pm 2.5$ & $24.2 \pm 3.3$ \\
\hline$W+b \bar{b}(2 b, \geq 1 \mathrm{tag})$ & & $48.6 \pm 3.2$ & $50.0 \pm 3.8$ & $50.3 \pm 4.9$ \\
\hline$W+b \bar{b}(2 b, \geq 2$ tags $)$ & & $9.1 \pm 1.4$ & $9.5 \pm 1.5$ & $8.1 \pm 1.4$ \\
\hline$W+c \bar{c}(1 c, \geq 1 \mathrm{tag})$ & $6.2 \pm 0.9$ & $6.7 \pm 1.0$ & $6.1 \pm 1.1$ & $7.7 \pm 1.9$ \\
\hline$W+c \bar{c}(2 c, \geq 1 \mathrm{tag})$ & & $12.3 \pm 1.9$ & $11.6 \pm 2.0$ & $10.1 \pm 2.3$ \\
\hline$W+c \bar{c}(2 c, \geq 2$ tags $)$ & & $0.5 \pm 0.2$ & $0.4 \pm 0.1$ & $0.8 \pm 0.4$ \\
\hline$W+c(\geq 1 \mathrm{tag})$ & $5.8 \pm 0.9$ & $6.1 \pm 0.9$ & $7.1 \pm 1.2$ & $5.6 \pm 1.6$ \\
\hline
\end{tabular}

Table 6.6: Ratio of $W+$ heavy flavor production to total $W+$ jets production for different jet multiplicities. The SecVtx event tagging efficiencies include the $b$-tagging scale factor of $0.909 \pm 0.060$. The samples of $W+b \bar{b}$ and $W+c \bar{c}$ are further divided into two classes based on the number of heavy flavor jets $\left(E_{T}>15 \mathrm{GeV}\right.$ and $|\eta|<2.4)$ inside the detector.

\begin{tabular}{ccccc}
\hline \hline Jet Multiplicity & 1jet & 2jets & 3jets & $\geq$ 4jets \\
\hline$W+b b$ & $99.3 \pm 34.2$ & $54.0 \pm 18.4$ & $10.5 \pm 3.5$ & $1.6 \pm 0.7$ \\
$W+c \bar{c}$ & $37.6 \pm 13.0$ & $19.5 \pm 6.6$ & $4.2 \pm 1.4$ & $0.7 \pm 0.3$ \\
$W+c$ & $83.2 \pm 20.9$ & $16.8 \pm 4.3$ & $2.2 \pm 0.6$ & $0.3 \pm 0.1$ \\
\hline \hline
\end{tabular}

Table 6.7: The estimated $W+$ heavy flavor background contributions.

The $t \bar{t}$ cross section group [58] in the CDF collaboration found, by fitting the entire jet multiplicity distribution, that the data are most compatible with $W+$ heavy flavor background; the distribution is $15 \pm 11 \%$ higher than the predicted contributions. No rescaling of any other background is favored. Obviously we cannot use this number blindly because it fits our signal region, namely the $W+2$ jets bin. Of all the jet multiplicity bins, the $W+1$ jet bin has the most statistical power to contribute to the fit. We fit to derive an independent a single heavy flavor rescaling factor using that $W+1$ jet bin. Our fitted rescaling value is $1.2 \pm 0.2$, consistent with what the top group found. However once this fit has been performed, the $W+1$ jet bin is no longer an independent control region for the background estimate. The results are shown in Table 6.7 . 


\begin{tabular}{cc}
\hline \hline Process & Cross Section $(\mathrm{pb})$ \\
\hline$W^{+} W^{-}$ & $12.4 \pm 0.80$ \\
$W Z^{0}$ & $3.96 \pm 0.06$ \\
$Z^{0} Z^{0}$ & $1.58 \pm 0.02$ \\
$t \bar{t}\left(m_{t}=173.5 \mathrm{GeV} / c^{2}\right)$ & $8.6 \pm 1.3$ \\
single top $(s$-channel $)$ & $0.88 \pm 0.05$ \\
single top $(t$-channel $)$ & $1.98 \pm 0.08$ \\
$Z^{0} \rightarrow \tau^{+} \tau^{-}$ & $254.9 \pm 5.6$ \\
\hline \hline
\end{tabular}

Table 6.8: The theoretical and measured cross sections used to estimate the MC derived background contributions.

\subsection{Other Backgrounds ( $t \bar{t}$, single top, $W^{+} W^{-}$, $W Z^{0}, Z^{0} Z^{0}$ and $\left.Z^{0} \rightarrow \tau^{+} \tau^{-}\right)$}

A number of backgrounds are too small to be measured from data, thus the simulation is used to predict their contribution to the sample. The diboson production processes $W^{+} W^{-}, W Z^{0}$ and $Z^{0} Z^{0}$, in association with jets, can mimic the $W H$ signal, when one boson decays leptonically and the other hadronically. The process $Z^{0} \rightarrow \tau^{+} \tau^{-}$, in association with jets, can mimic the signal, when one $\tau$ decays leptonically and the other decays hadronically. The $t$ quark decays into $W^{+}$boson and $b$ quark, where $W^{+}$boson decays leptonically or hadronically. As the result, $t \bar{t}$ and single top ( $s$-channel $q \bar{q}$ annihilation and $t$-channel $W$-gluon fusion) processes can also mimic the signal.

MC samples are used to measure the acceptance and $b$-tagging efficiency. The $\mathrm{MC}$ acceptances are corrected for the lepton identification and trigger efficiencies as described in Chapter 7 . The $b$-tagging efficiency is also scaled by the factor of $0.909 \pm 0.060$, described in Section 5.5. The normalization is based on the measured integrated luminosity and the theoretical (single top, $W^{+} W^{-}, W Z^{0}$ and $Z^{0} Z^{0}$ ) and measured $\left(t \bar{t}\right.$ and $\left.Z^{0} \rightarrow \tau^{+} \tau^{-}\right)$cross sections, shown in Table 6.8 [60, 61, 62, 63, 64].

\subsection{Summary of Background Estimation}

Figure 6.2 and Table 6.9 summarize the number of observed $W+b$-tagged events along with the estimated background events. The number of observed $W+b$-tagged events in $W+1$ jet and $W+\geq 3$ jets are used to constrain the $W+$ heavy flavor and $t \bar{t}$ backgrounds. 


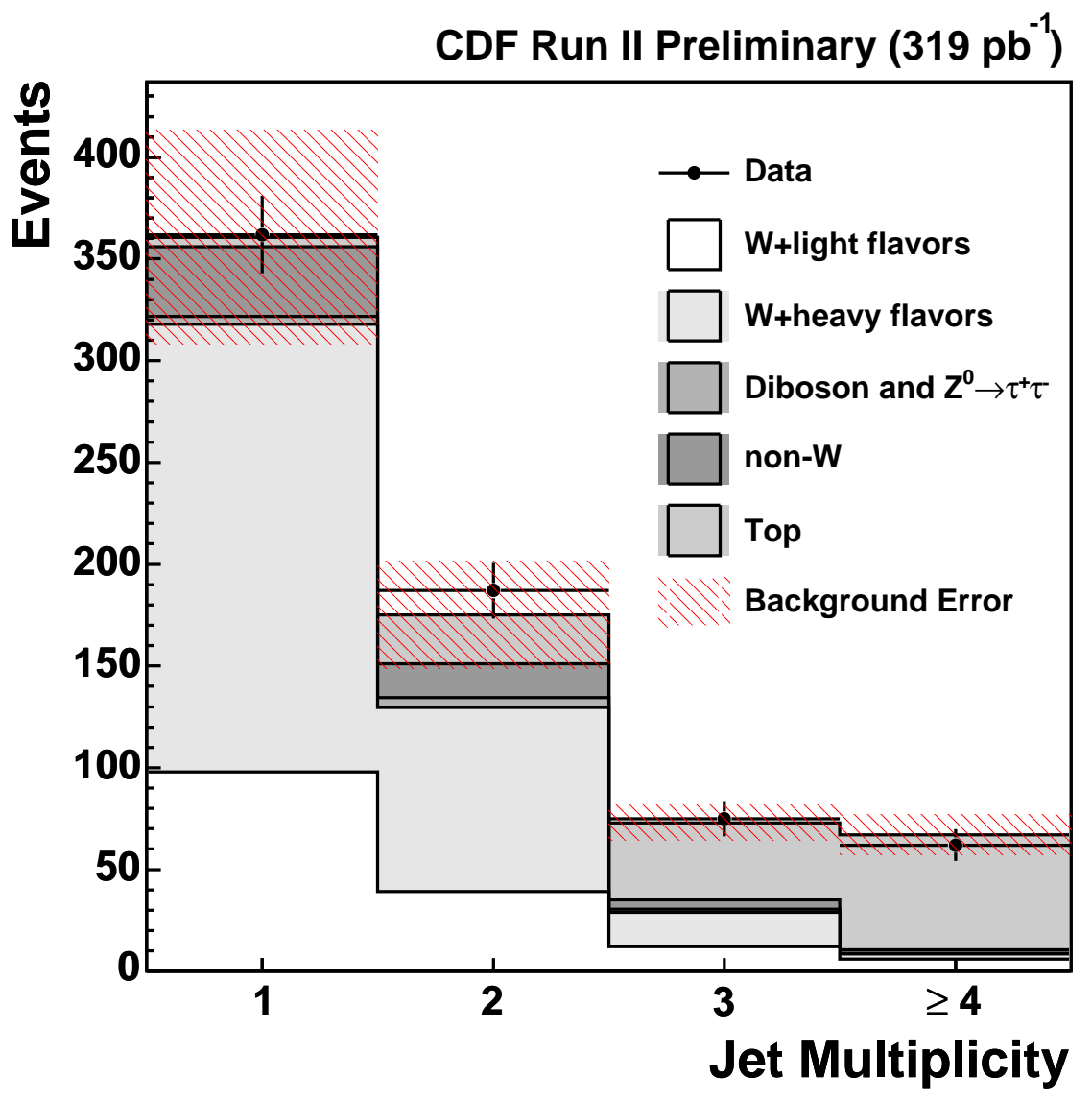

Figure 6.2: The number of observed $W+$ positive tagged events and the background summary as a function of jet multiplicity. The integrated luminosities are $318.5 \mathrm{pb}^{-1}$ for CEM and CMUP, and $305.2 \mathrm{pb}^{-1}$ for CMX.

\begin{tabular}{ccccc}
\hline \hline Background & $W+1$ jet & $W+2$ jets & $W+3$ jets & $W+\geq 4$ jets \\
\hline Events before tagging & 26218 & 3910 & 602 & 160 \\
\hline Mistags & $98.0 \pm 7.9$ & $39.3 \pm 3.1$ & $12.1 \pm 1.2$ & $5.9 \pm 0.8$ \\
$W+b \bar{b}$ & $99.3 \pm 34.2$ & $54.0 \pm 18.4$ & $10.5 \pm 3.5$ & $1.6 \pm 0.7$ \\
$W+c \bar{c}$ & $37.6 \pm 13.0$ & $19.5 \pm 6.6$ & $4.2 \pm 1.4$ & $0.7 \pm 0.3$ \\
$W+c$ & $83.2 \pm 20.9$ & $16.8 \pm 4.3$ & $2.2 \pm 0.6$ & $0.3 \pm 0.1$ \\
Diboson $/ Z^{0} \rightarrow \tau^{+} \tau^{-}$ & $3.7 \pm 0.9$ & $5.0 \pm 1.1$ & $1.5 \pm 0.5$ & $0.3 \pm 0.1$ \\
non- $W$ & $34.3 \pm 6.3$ & $16.5 \pm 3.2$ & $4.8 \pm 1.0$ & $1.9 \pm 0.4$ \\
single top & $3.4 \pm 0.7$ & $9.6 \pm 2.0$ & $2.0 \pm 0.5$ & $0.4 \pm 0.1$ \\
$t \bar{t}$ & $1.3 \pm 0.2$ & $14.6 \pm 2.5$ & $35.6 \pm 6.0$ & $56.2 \pm 9.5$ \\
\hline Total Background & $360.7 \pm 52.7$ & $175.2 \pm 26.3$ & $72.8 \pm 8.7$ & $67.1 \pm 9.9$ \\
\hline Observed $W+$ positive tags & 362 & 187 & 75 & 62 \\
\hline \hline
\end{tabular}

Table 6.9: The number of observed $W+$ positive tagged events and the background summary for an integrated luminosity of $318.5 \mathrm{pb}^{-1}$ for CEM and CMUP, and 305.2 $\mathrm{pb}^{-1}$ for CMX. 



\section{Chapter 7}

\section{Search for the Higgs Boson Decaying into $b \bar{b}$ and Produced in Association with $W$ Boson}

This chapter describes a search for the Higgs boson decaying into $b \bar{b}$ and produced in association with $W$ boson using $319 \mathrm{pb}^{-1}$ dataset. The search starts from event selection optimization for jet $E_{T}$ selection criteria, described in Section 7.1. Section 7.2 describes the reconstructed dijet mass distribution, the mass width, the mass deviation and the mass resolution for the Higgs boson. Section 7.3 describes the dijet mass and $W$ transverse mass shapes for the non- $W$ background. After validation of kinematic distributions (Section 7.4), we discuss the validation of the dijet mass shape using $W+\geq 3$ jets bin in Section 7.5. Finally we search for a dijet invariant mass resonance from the Higgs boson in the reconstructed dijet mass distribution after $b$-tagging and discuss the validation of the dijet mass shape in $W+2$ jets bin (Section 7.6).

\subsection{Event Selection Optimization}

The 1st and 2nd leading jet $E_{T}$ selection criteria are studied in order to maximize the search sensitivity. We vary the cuts from $15 \mathrm{GeV}$ to $50 \mathrm{GeV}$ using a $W H \mathrm{MC}$ sample and the same background estimation methods as described in Chapter 6 . We evaluate the significance for each set of cuts. The significance is defined as $S / \sqrt{B}$, where $S$ and $B$ are the numbers of signal and background events in a mass window, which is within $\pm 1.5 \sigma_{\text {rec }}$ of the reconstructed Higgs boson mass, where the $\sigma_{\text {rec }}$ shows the width of the dijet mass fit by Gaussian. Figure 7.1 shows the significance as a function of the jet $E_{T}$ selection criteria. Since the significance is not sensitive to the 1st leading jet $E_{T}$ and jet $E_{T}>15 \mathrm{GeV}$ selection criteria are well studied in the CDF collaboration [58], we require the 1st leading jet $E_{T}>15 \mathrm{GeV}$ and the $2 \mathrm{nd}$ leading jet $E_{T}>15 \mathrm{GeV}$. 

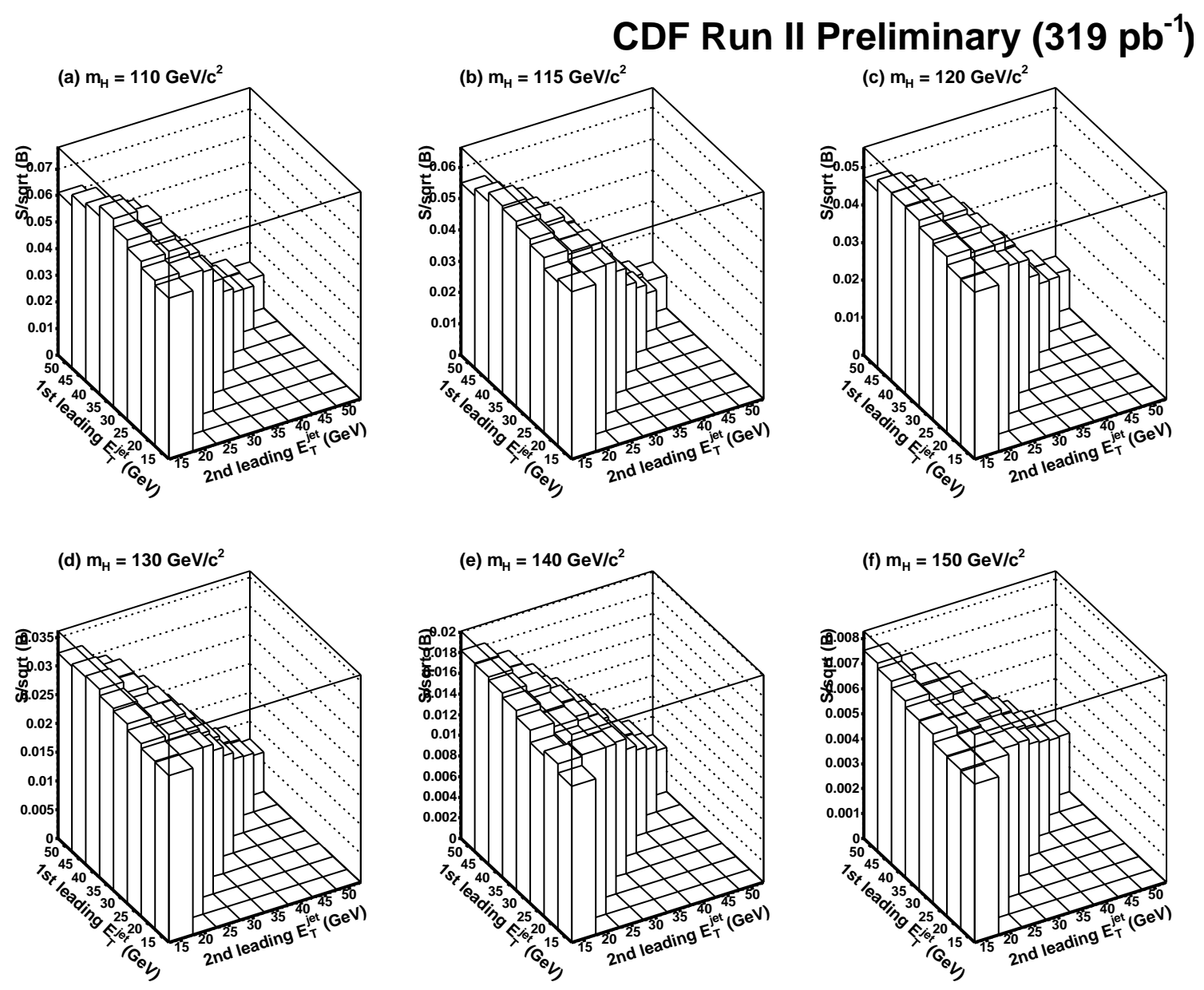

Figure 7.1: The significance as a function of 1st and 2nd leading jet $E_{T}$ selection criteria. The significance is not sensitive to the 1st leading jet $E_{T}$. (a) $m_{H}=$ $110 \mathrm{GeV} / c^{2}$, (b) $m_{H}=115 \mathrm{GeV} / c^{2}$, (c) $m_{H}=120 \mathrm{GeV} / c^{2}$, (d) $m_{H}=130 \mathrm{GeV} / c^{2}$, (e) $m_{H}=140 \mathrm{GeV} / c^{2}$, (f) $m_{H}=150 \mathrm{GeV} / c^{2}$. 


\subsection{Dijet Mass Distribution for the Higgs Boson}

Since the Higgs boson decays into two $b$ quark jets, it is important to understand the reconstructed dijet mass distribution for the Higgs boson. Figure 7.2 shows the jet multiplicity distribution after event selections using $W H \mathrm{MC}$ sample of $m_{H}=115$ $\mathrm{GeV} / c^{2}$. The $W+2$ jets signature contains most of the signal events. We reconstruct the Higgs boson mass from two jets in $W+2$ jets bin. Figure 7.3 shows the dijet mass distributions. The dijet mass distributions have the low mass tails which originate from events with $b$ semileptonic decay and events with the gluon jets ${ }^{1}$. We fit the distributions with Gaussians in $2 \sigma_{\text {rec }}$ mass regions. Then we define the mass deviation and the mass resolution as $\left(m_{\text {rec }}-m_{\text {gen }}\right) / m_{\text {gen }}$ and $\sigma_{\text {rec }} / m_{\text {rec }}$, where $m_{\text {rec }}$ and $m_{\text {gen }}$ denote the Gaussian central value and Higgs mass we set in PYTHIA. Figure 7.4 shows the central value and width of Gaussian fit, the mass deviation and the mass resolution. Figures 7.5 to 7.9 show the kinematic distributions for lepton $E_{T}, E_{T}, W$ transverse mass, 1 st leading jet $E_{T}$ and 2 nd leading jet $E_{T}$ in the tagged samples, respectively, where $m_{H}=115 \mathrm{GeV} / c^{2}$ is assumed.

\subsection{Dijet Mass and $W$ Transverse Mass Shapes for Non- $W$ Background}

Since there is no reliable MC simulation for non- $W$ background, we have to obtain predictions for this background directly from data. We have a reasonable method to estimate the number of non- $W$ background in the signal region, but characterizing kinematic distributions of non- $W$ background in the signal region $(I<0.1$ and $E_{T}>20 \mathrm{GeV}$ ) may be difficult; the shape from one of the sidebands and the shape of events with the electron identified as one of photon conversion pair may be different. We have compared the shapes of dijet mass and $W$ transverse mass in the Regions $\mathrm{C}, \mathrm{A}^{\prime}$ and B' along with a dedicated sample of conversions in signal region. In Figure 7.10, the electron +2 jets sample is used for the dijet mass shapes in Regions $\mathrm{C}, \mathrm{A}^{\prime}$ and B, where the upper isolation cut is lowered from 0.2 to 0.1 for Region C. The dijet mass shapes are quite similar, but there seems to be some bias on the $W$ transverse mass due to different isolation and $\mathbb{E}_{T}$ requirements and additional photon conversion requirement. In particular, the isolation cut, $I>0.1$, selects a large number of events with the $W^{ \pm}$transverse mass near $0 \mathrm{GeV} / c^{2}$, where the non-isolated electron and $\mathbb{E}_{T}$ vector are in the same jet. For this reason, the Region B' ( $B_{T}$ sideband region) seems a better choice for kinematic modeling of the signal region. This approach is confirmed from the comparisons of the predicted dijet mass distribution with the distribution from pretag data events. Figure 7.11 shows the comparison with the $\chi^{2}$ test and Kolmogorov-Smirnov test (see Appendix B). The Region B' shape represents a quantitatively better agreement with data. We note that the $W$ transverse mass prediction is biased low in the $\mathbb{E}_{T}$ sideband region we used, but that the region does describe better the dijet mass distribution; the Higgs

\footnotetext{
${ }^{1}$ The gluon jets originate from initial and final state radiation.
} 


\section{CDF Run II Preliminary}
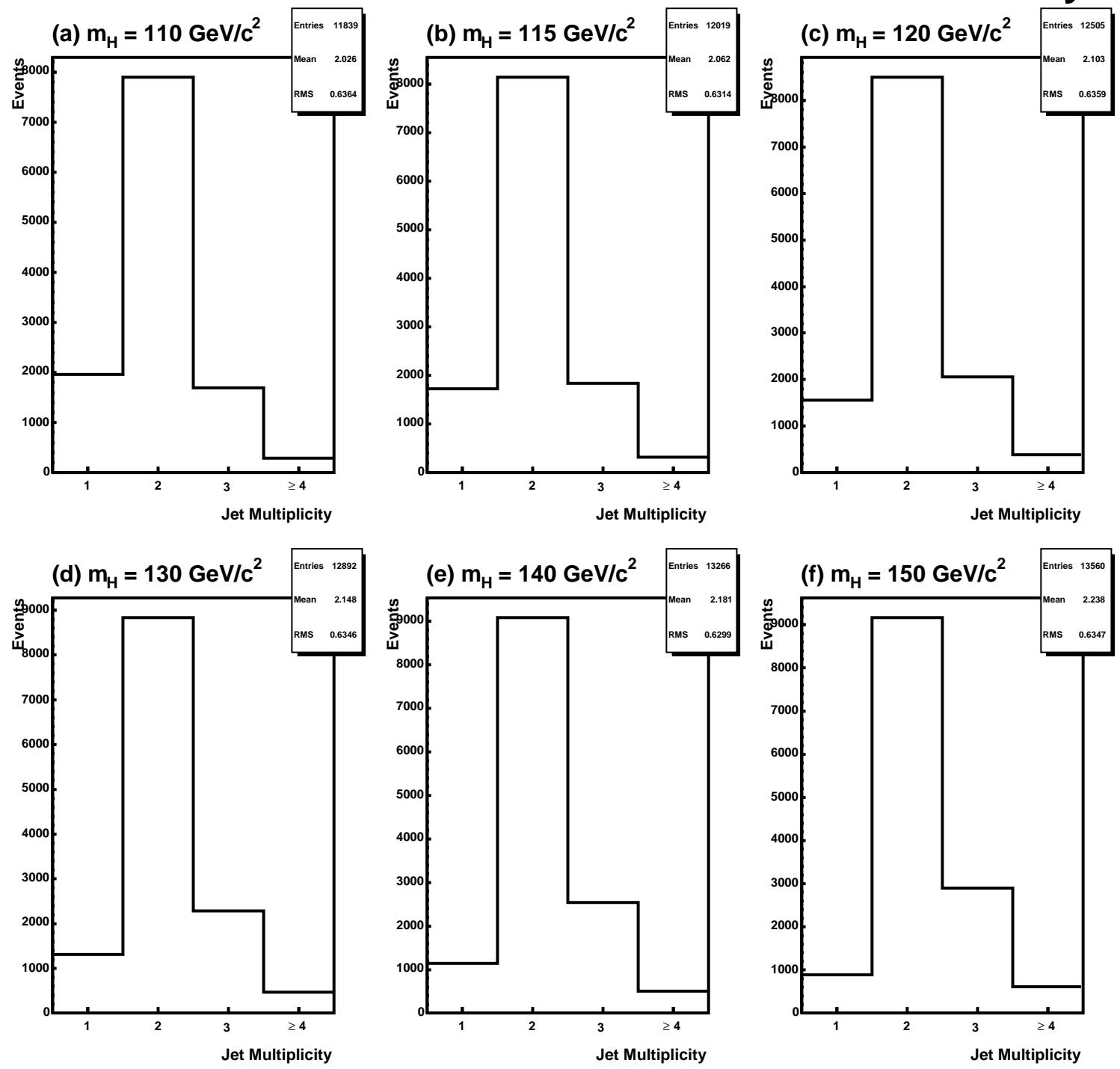

Figure 7.2: The jet multiplicity distribution after event selections using $W H \mathrm{MC}$ samples. (a) $m_{H}=110 \mathrm{GeV} / c^{2}$, (b) $m_{H}=115 \mathrm{GeV} / c^{2}$, (c) $m_{H}=120 \mathrm{GeV} / c^{2}$, (d) $m_{H}=130 \mathrm{GeV} / c^{2}$, (e) $m_{H}=140 \mathrm{GeV} / c^{2}$, (f) $m_{H}=150 \mathrm{GeV} / c^{2}$. The $W+2$ jets signature contains most of the signal events. 

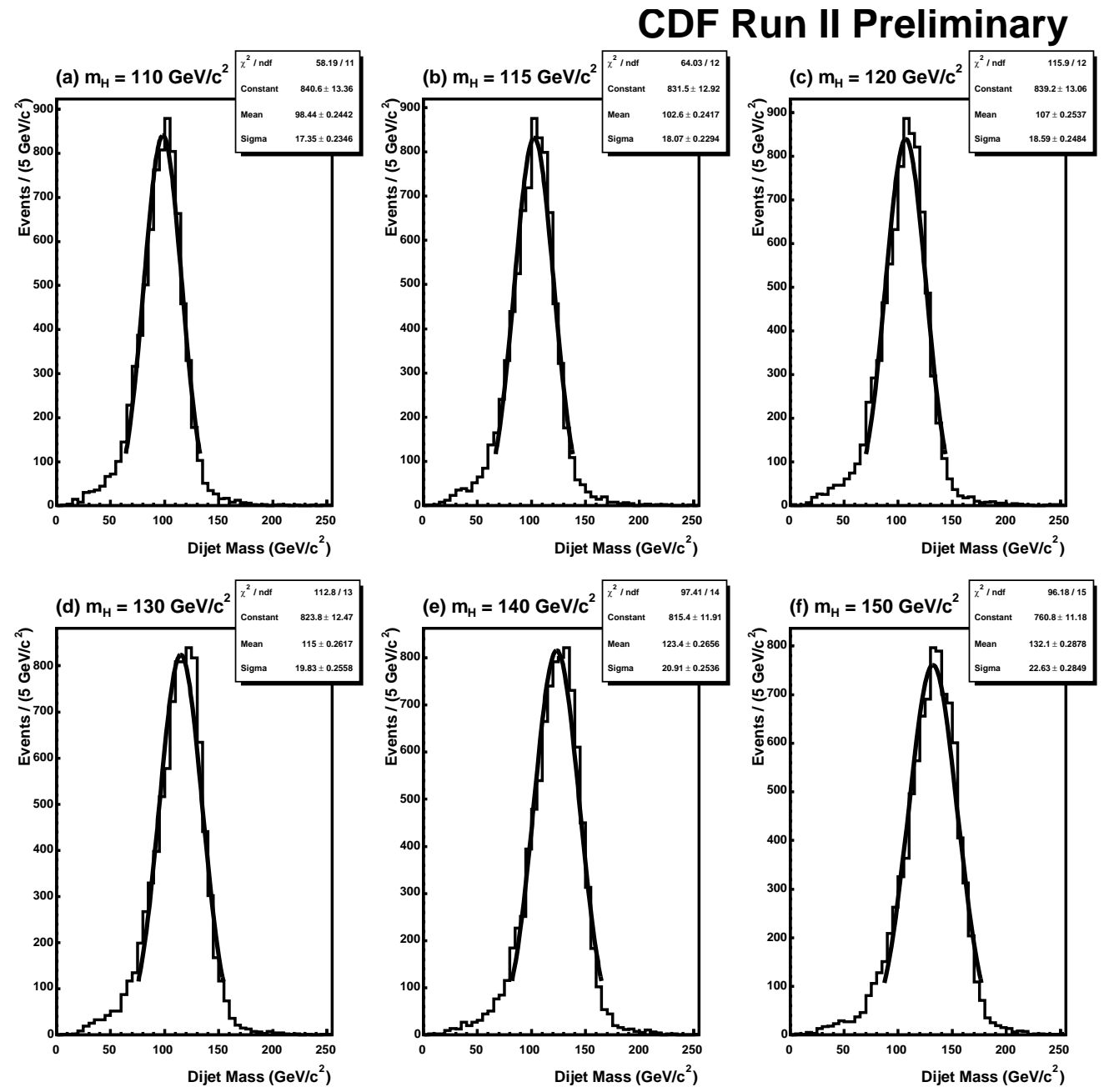

Figure 7.3: The reconstructed dijet mass distributions for the Higgs boson using $W H$ MC samples in $W+2$ jets bin. (a) $m_{H}=110 \mathrm{GeV} / c^{2}$, (b) $m_{H}=115 \mathrm{GeV} / c^{2}$, (c) $m_{H}=120 \mathrm{GeV} / c^{2}$, (d) $m_{H}=130 \mathrm{GeV} / c^{2}$, (e) $m_{H}=140 \mathrm{GeV} / c^{2}$, (f) $m_{H}=150$ $\mathrm{GeV} / c^{2}$.

boson search relies on not the $W$ transverse mass distribution but the dijet mass distribution. 

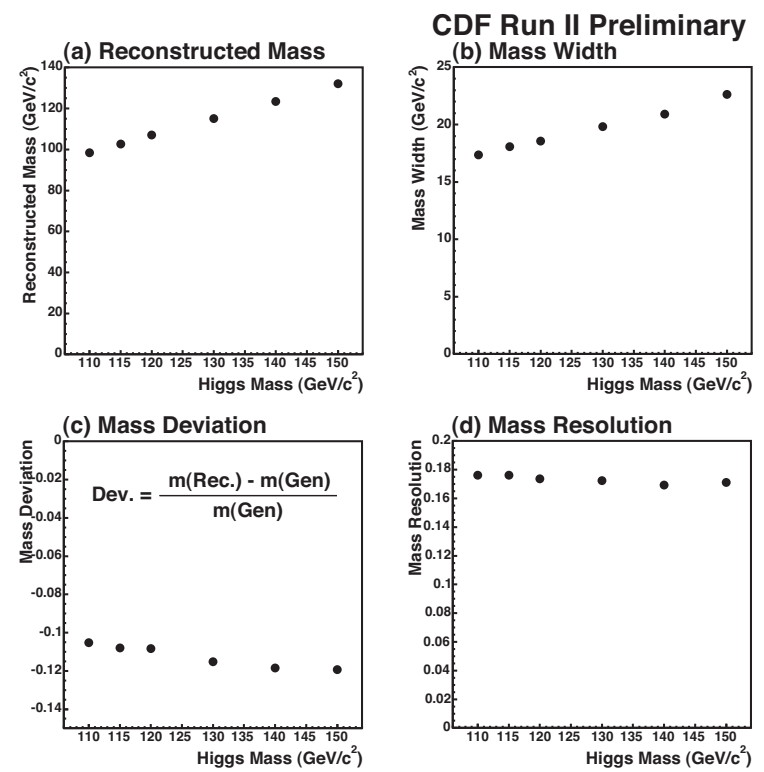

Figure 7.4: The Gaussian central value and width, the mass deviation and the mass resolution. (a)Gaussian central value, (b)Gaussian width, (c)mass deviation, (d)mass resolution.
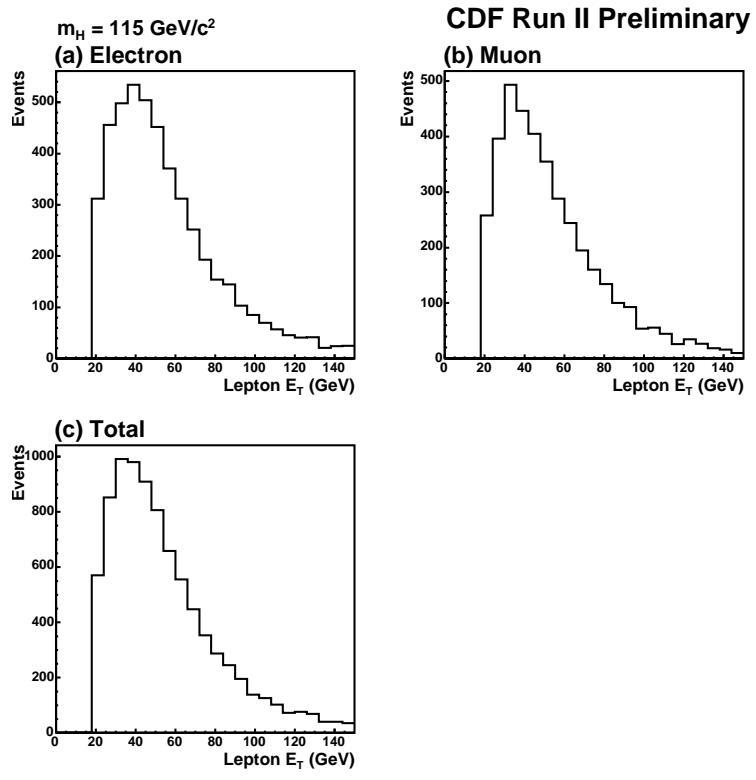

Figure 7.5: The lepton $E_{T}$ distributions for the $W H$ tagged sample, where $m_{H}=115$ $\mathrm{GeV} / c^{2}$ is assumed. (a)electron channel, (b)muon channel, (c)electron + muon channel. 

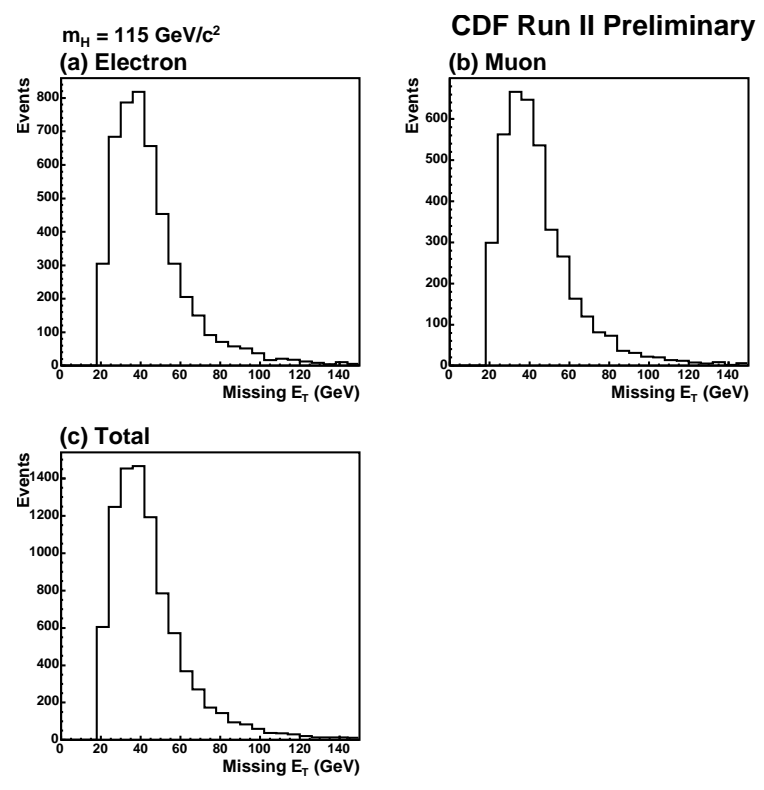

Figure 7.6: The $\not_{T}$ distributions for the $W H$ tagged sample, where $m_{H}=115$ $\mathrm{GeV} / c^{2}$ is assumed. (a)electron channel, (b)muon channel, (c)electron + muon channel.
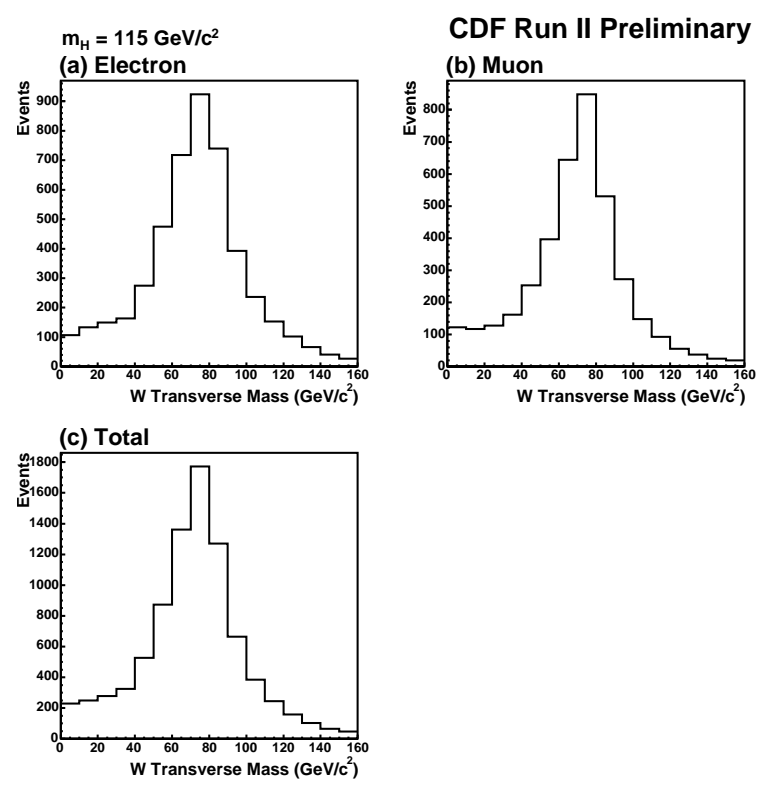

Figure 7.7: The $W$ transverse mass distributions for the $W H$ tagged sample, where $m_{H}=115 \mathrm{GeV} / c^{2}$ is assumed. (a)electron channel, (b)muon channel, (c)electron + muon channel. 

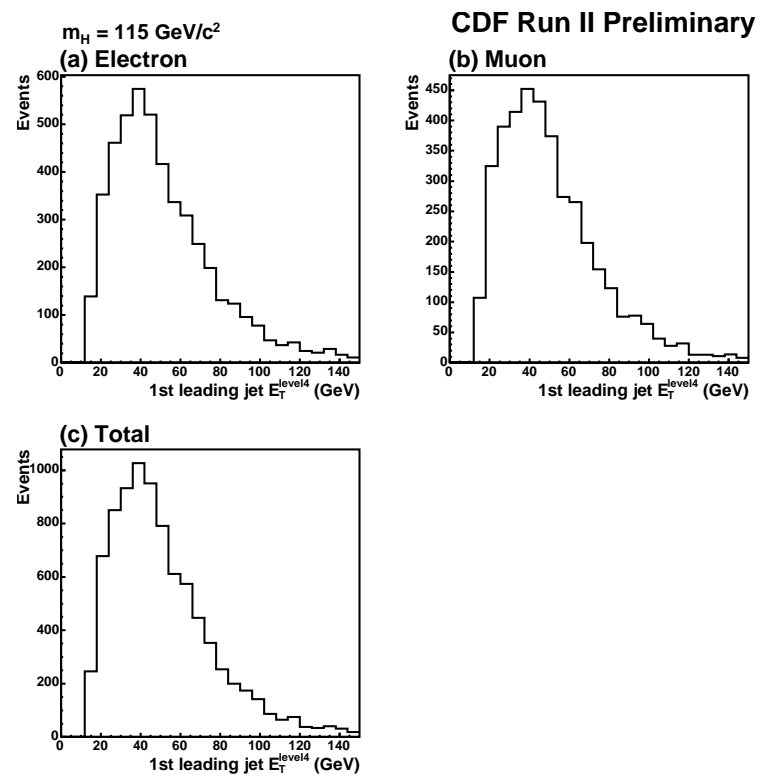

Figure 7.8: The 1st leading jet $E_{T}$ distributions for the $W H$ tagged sample, where $m_{H}=115 \mathrm{GeV} / c^{2}$ is assumed. (a)electron channel, (b)muon channel, (c)electron + muon channel.
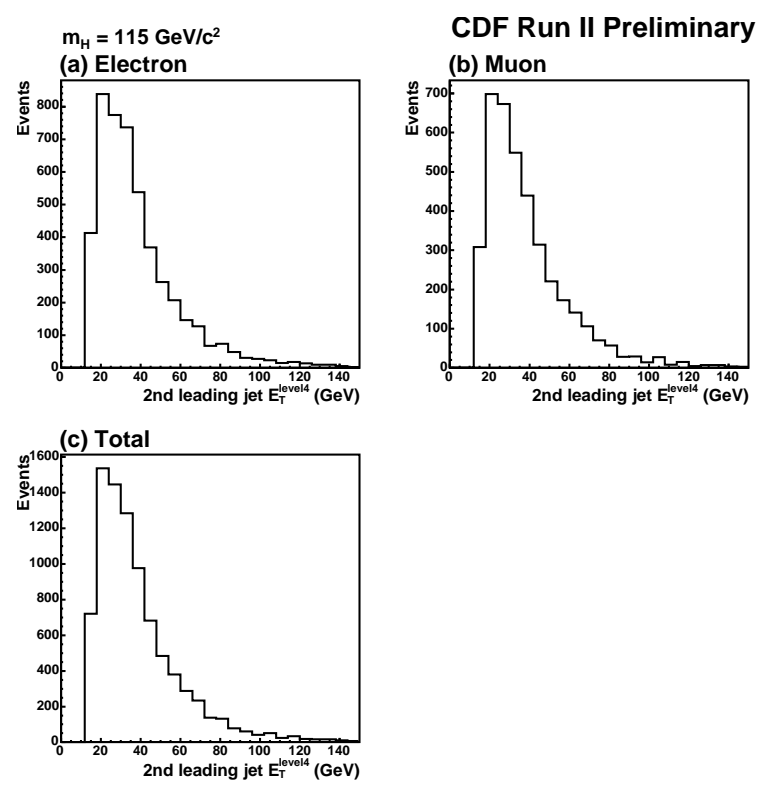

Figure 7.9: The 2nd leading jet $E_{T}$ distributions for the $W H$ tagged sample, where $m_{H}=115 \mathrm{GeV} / c^{2}$ is assumed. (a)electron channel, (b)muon channel, (c)electron + muon channel. 

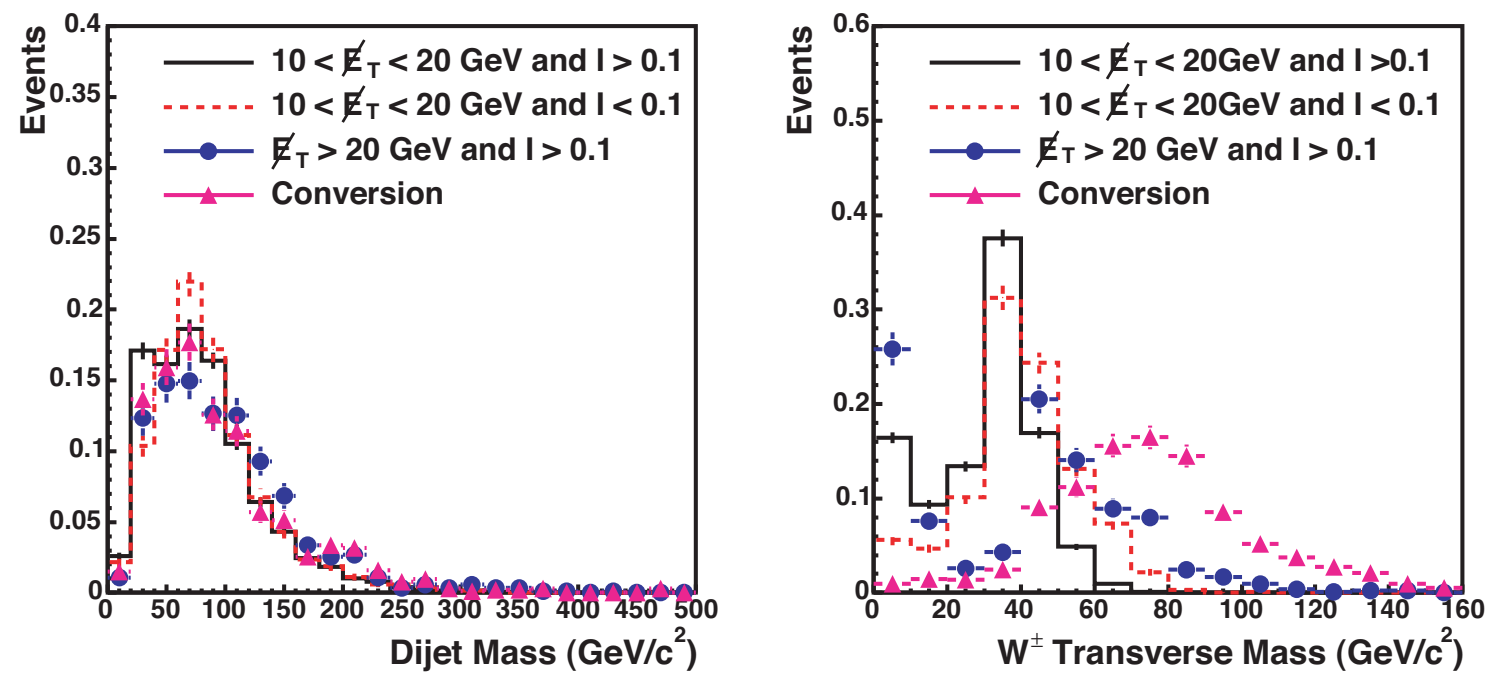

Figure 7.10: The comparisons of dijet and $W$ transverse mass in the sideband Regions $\mathrm{C}, \mathrm{A}^{\prime}, \mathrm{B}$ ' and in the conversion sample, where the upper isolation cut is lowered from 0.2 to 0.1 for Region C. (Left)dijet mass distribution, (Right) $W$ transverse mass distribution.
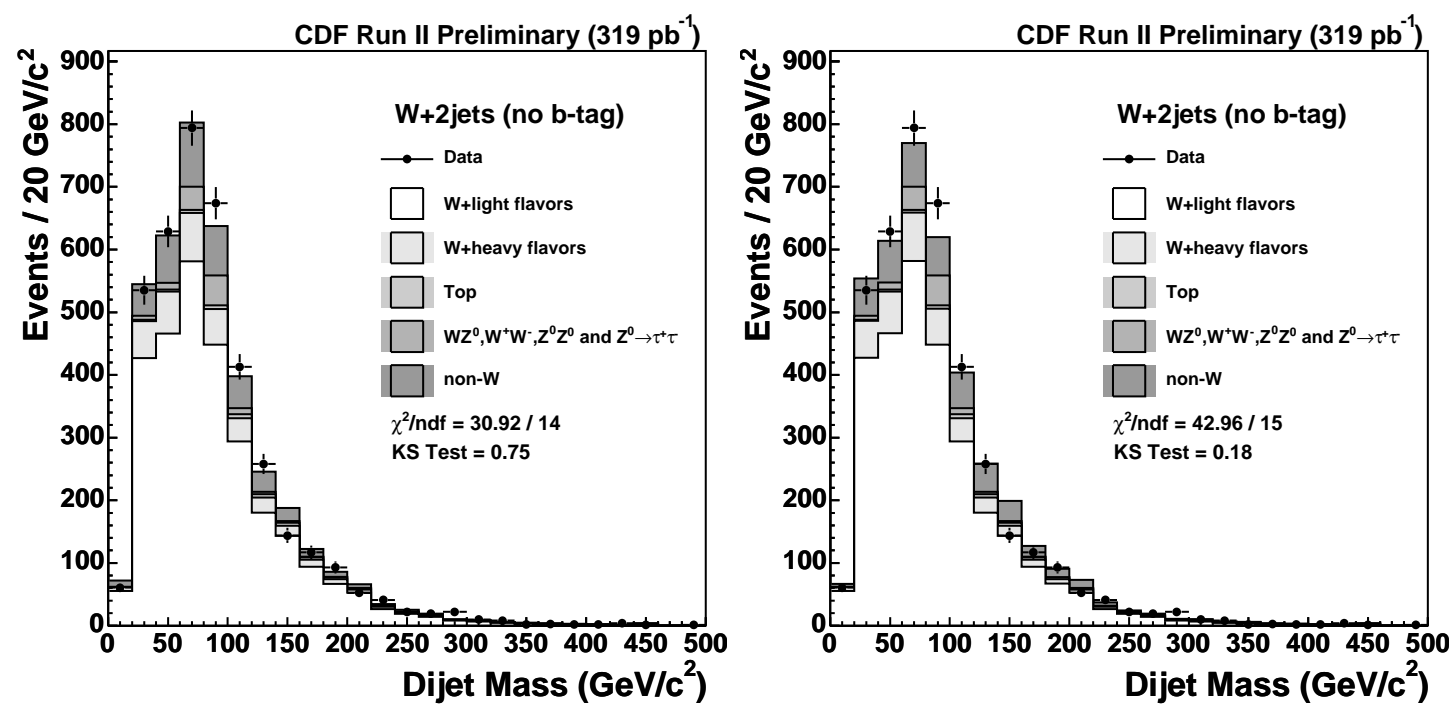

Figure 7.11: The comparisons of pretag dijet mass prediction with data events. The plot on the left uses a non- $W$ dijet mass shape derived in Region B', and the plot on the right uses a non- $W$ dijet mass shape derived in Region $\mathrm{C}$, where the upper isolation cut is lowered from 0.2 to 0.1 for Region C. 


\subsection{Validation of Kinematic Distributions}

Before proceeding to the Higgs boson search, we validate some of basic kinematic distributions using a large control sample of $W+2$ jets and $b$-tagged $W+1$ jet in both data and MC samples. This is to insure our search for new physics in any samples with limited statistics, such as in the $b$-tagged $W+2$ jets sample we are using in this analysis. We have compared the kinematic distributions for pretag and tagged samples. Figures 7.12 to 7.25 show the kinematic distributions for lepton $E_{T}, \mathbb{E}_{T}, W$ transverse mass, 1 st leading jet $E_{T}$ and 2nd leading jet $E_{T}$ in the $W+2$ jets pretag and tagged samples, and in the $W+1$ jet tagged sample, respectively ${ }^{2}$. The data seem to be well described by the MC, except in some of the distributions for the $W+2$ jets tagged sample, where statistical significance is limited.
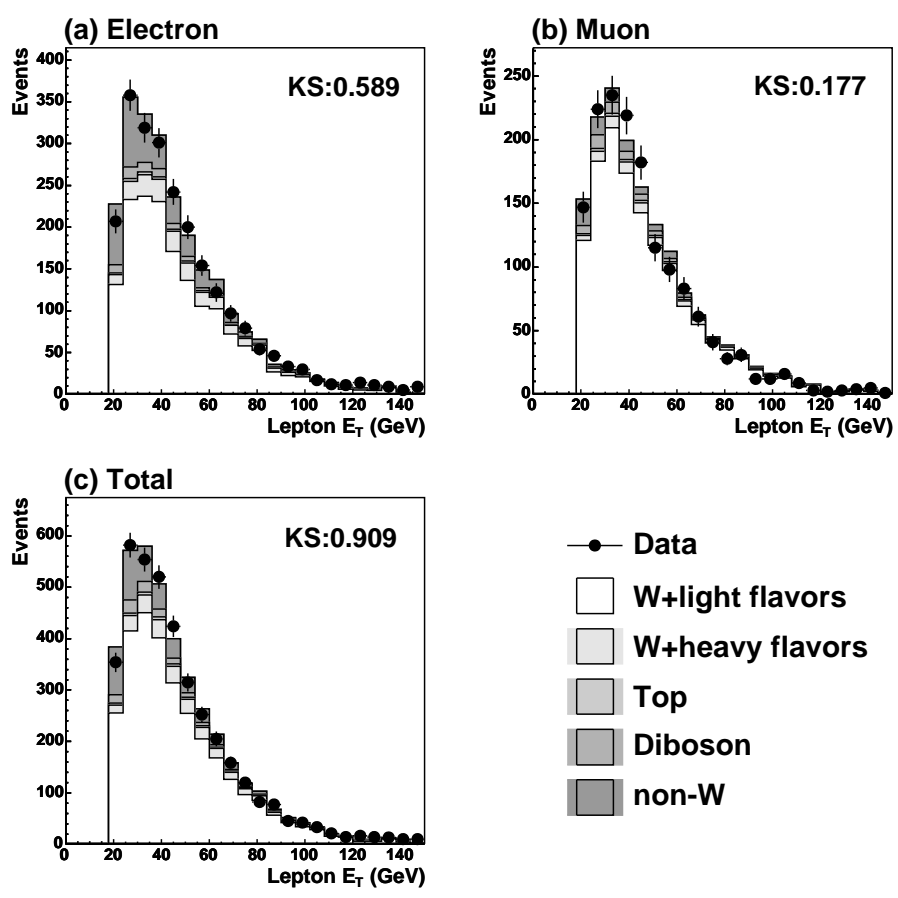

Figure 7.12: The lepton $E_{T}$ distributions for $W+2$ jets pretag sample. (a)electron channel, (b)muon channel, (c)electron + muon channel.

\subsection{Dijet Mass Shape}

To understand the dijet mass resolution, we compare the observed dijet mass distribution with the expected dijet mass distribution. This is required to extract the limit on the Higgs boson production, if there is no significant dijet invariant mass resonance from the Higgs boson.

\footnotetext{
${ }^{2} \mathbb{E}_{T}>20 \mathrm{GeV}$ and $I>0.1$ region is utilized for the $\mathbb{E}_{T}$ distribution.
} 

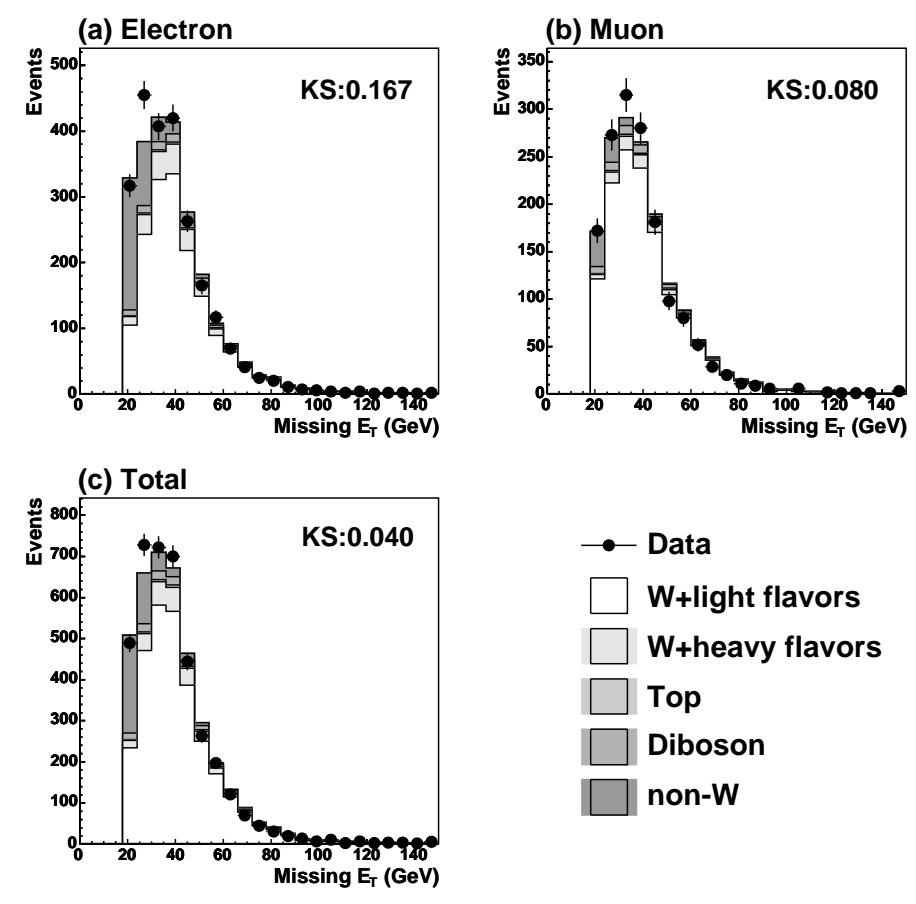

Figure 7.13: The $\mathbb{E}_{T}$ distributions for $W+2$ jets pretag sample. (a)electron channel, (b)muon channel, (c)electron + muon channel.

We checked the dijet invariant mass distribution of the two leading jets in the $b$-tagged $W+\geq 3$ jets events where $t \bar{t}$ contribution dominates. Figure 7.26 exhibits a good agreement between data and expectation, which gives us a confidence in the validity of the simulation and background modeling.

We have also compared the pretag dijet mass distribution with the expectation from the various backgrounds, which is shown in Figure 7.11 (Left). The agreement between the data and $\mathrm{MC}$ is reasonable again.

\subsection{Dijet Mass Distribution}

We perform a direct search for a resonant mass peak in the reconstructed dijet invariant mass distribution. Figure 7.27 shows the dijet mass distribution after requiring at least one $b$-tagged jet in $W+2$ jet sample. There seems to be an excess of events between 40 and $120 \mathrm{GeV} / c^{2}$, but it is not statistically significant. Figure 7.28 shows the ratio of the numbers of events between data and background expectation as a function of the dijet mass for the pretag and tagged samples. The errors include statistical errors only from the data sample. The ratios are consistent to be unity, fluctuating around 1 . 

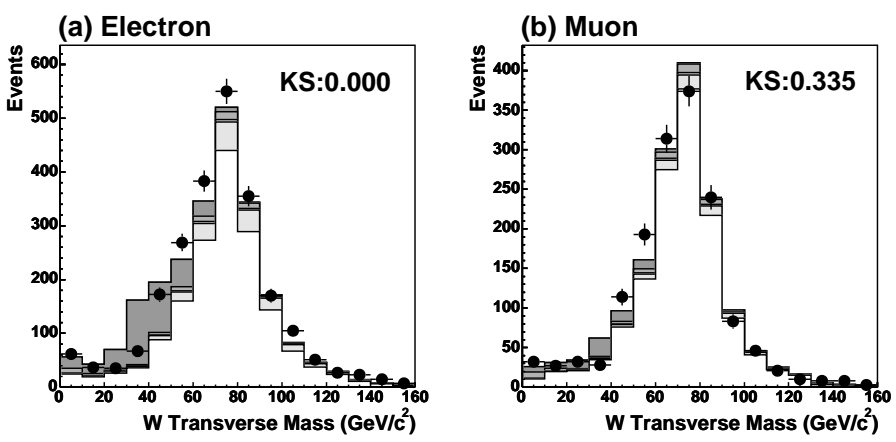

\section{(c) Total}
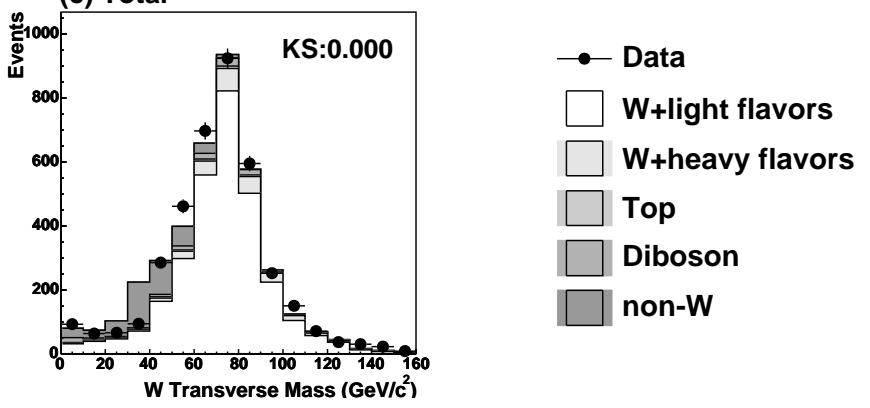

Figure 7.14: The $W$ transverse mass distributions for $W+2$ jets pretag sample. (a)electron channel, (b)muon channel, (c)electron + muon channel.

(a) Electron
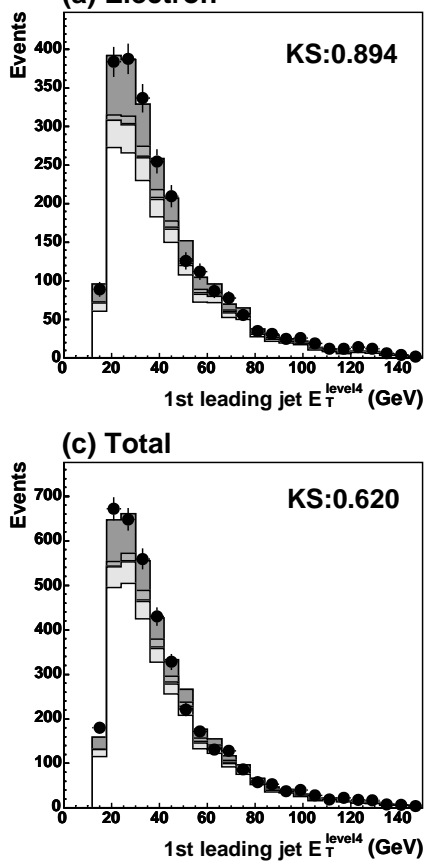

(b) Muon
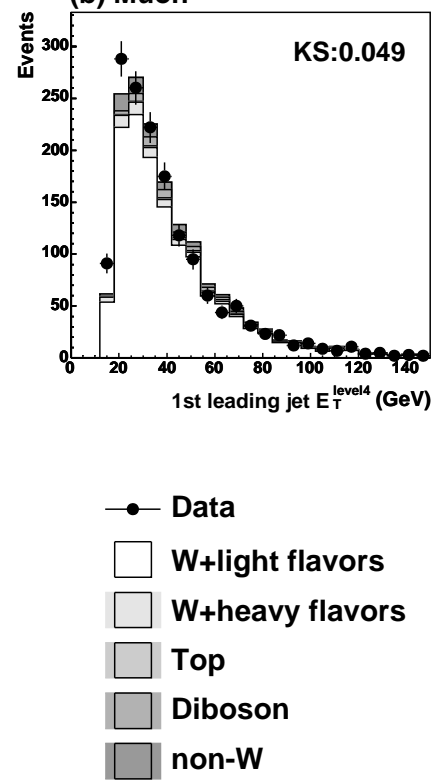

Figure 7.15: The 1st leading jet $E_{T}$ distributions for $W+2$ jets pretag sample. (a)electron channel, (b)muon channel, (c)electron + muon channel. 

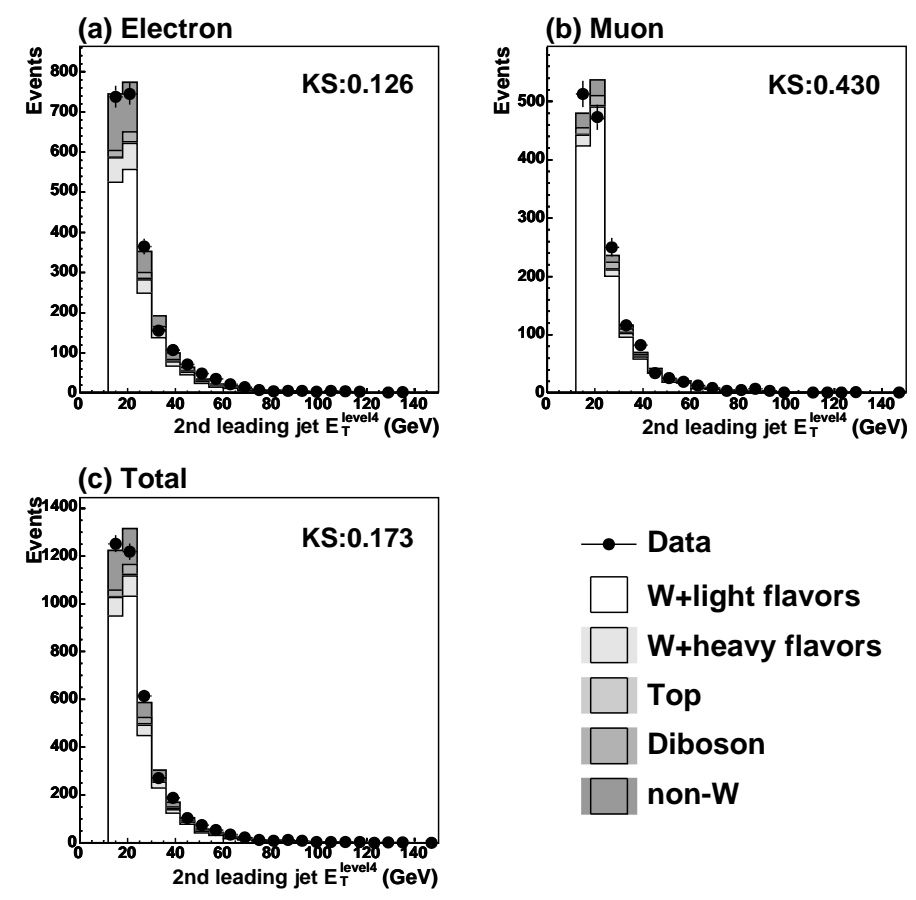

Figure 7.16: The 2nd leading jet $E_{T}$ distributions for $W+2$ jets pretag sample. (a)electron channel, (b)muon channel, (c)electron + muon channel.
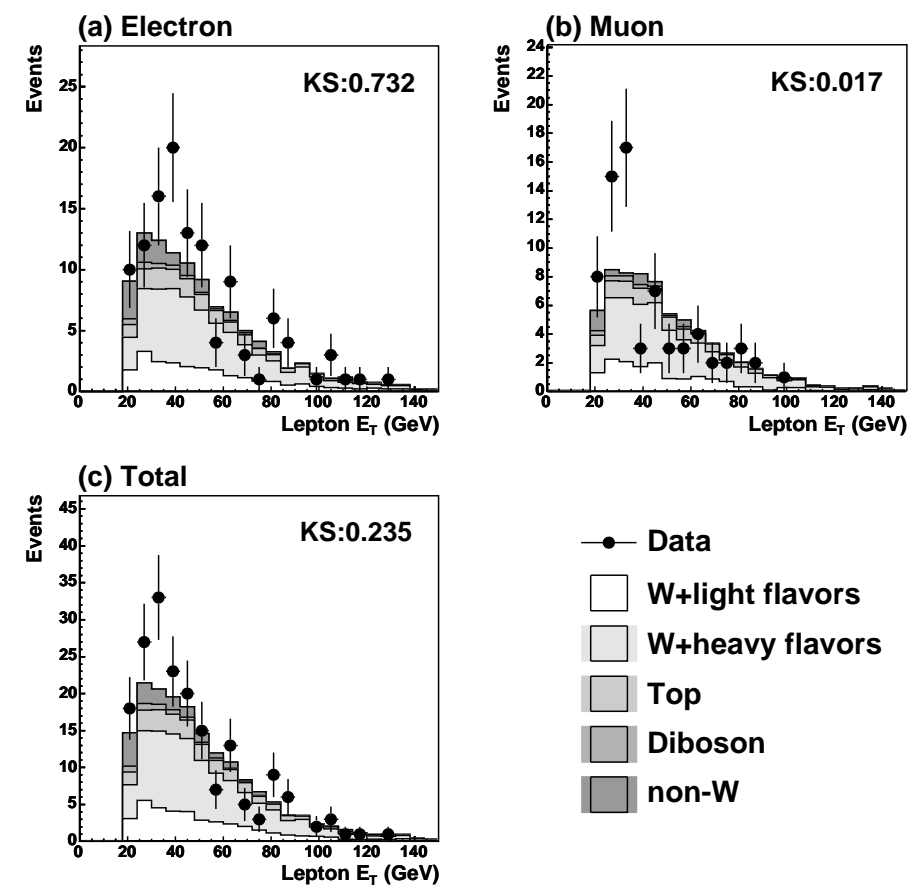

Figure 7.17: The lepton $E_{T}$ distributions for $W+2$ jets tagged sample. (a)electron channel, (b)muon channel, (c)electron + muon channel. 

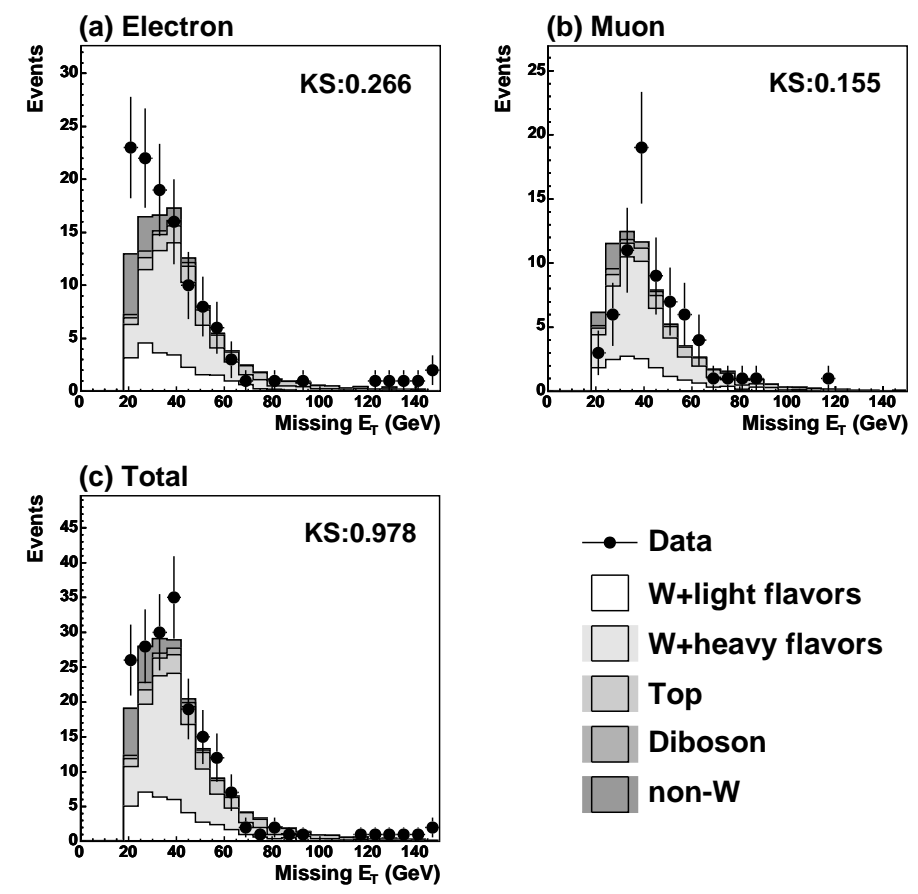

Figure 7.18: The $\mathbb{E}_{T}$ distributions for $W+2$ jets tagged sample. (a)electron channel, (b)muon channel, (c)electron + muon channel.

(a) Electron
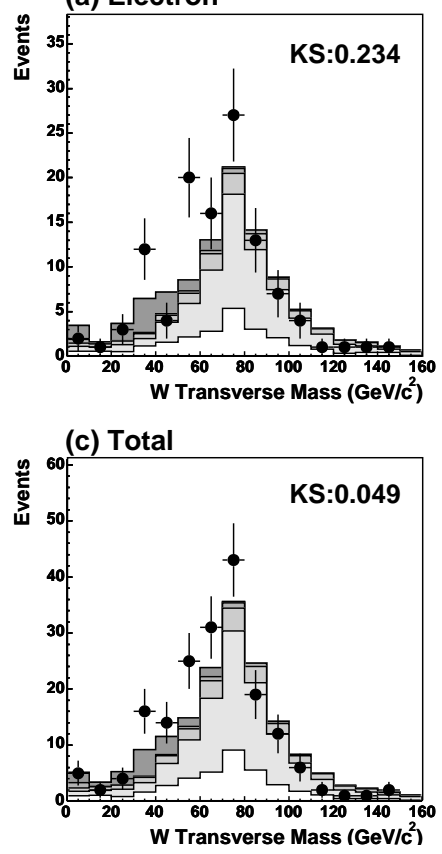

(b) Muon

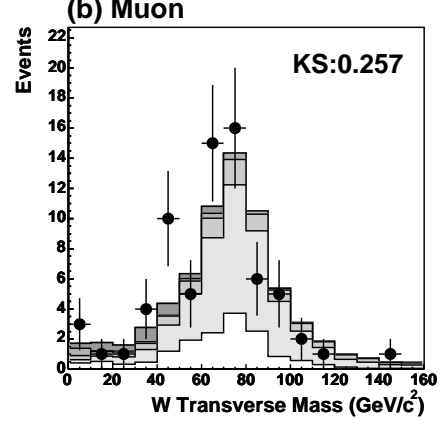

$\multimap$ Data

W+light flavors

W+heavy flavors

Top

Diboson

non-W

Figure 7.19: The $W$ transverse mass distributions for $W+2$ jets tagged sample. (a)electron channel, (b)muon channel, (c)electron + muon channel. 
(a) Electron

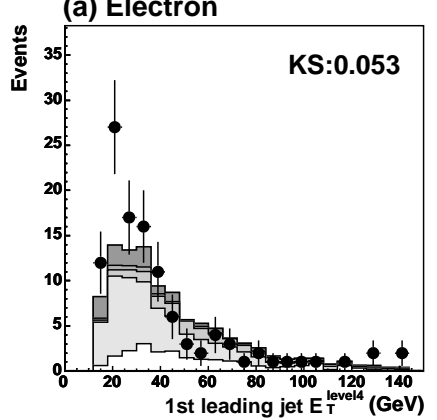

(c) Total

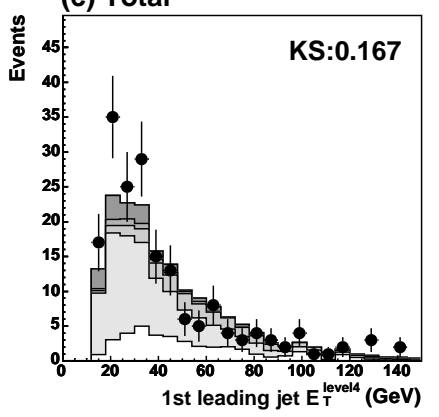

(b) Muon
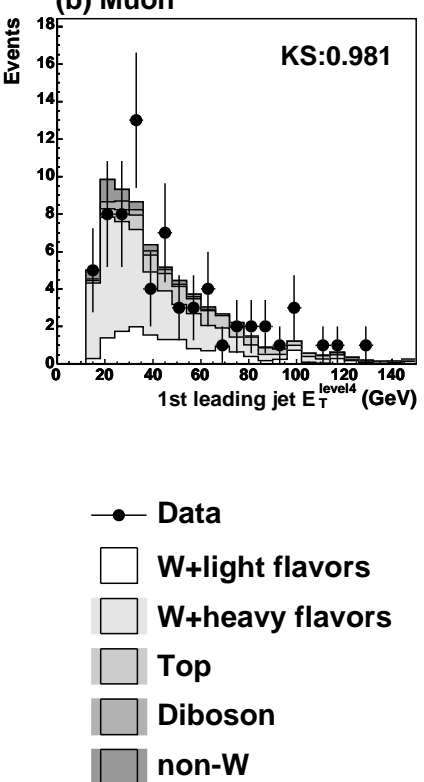

Figure 7.20: The 1st leading jet $E_{T}$ distributions for $W+2$ jets tagged sample. (a)electron channel, (b)muon channel, (c)electron + muon channel.
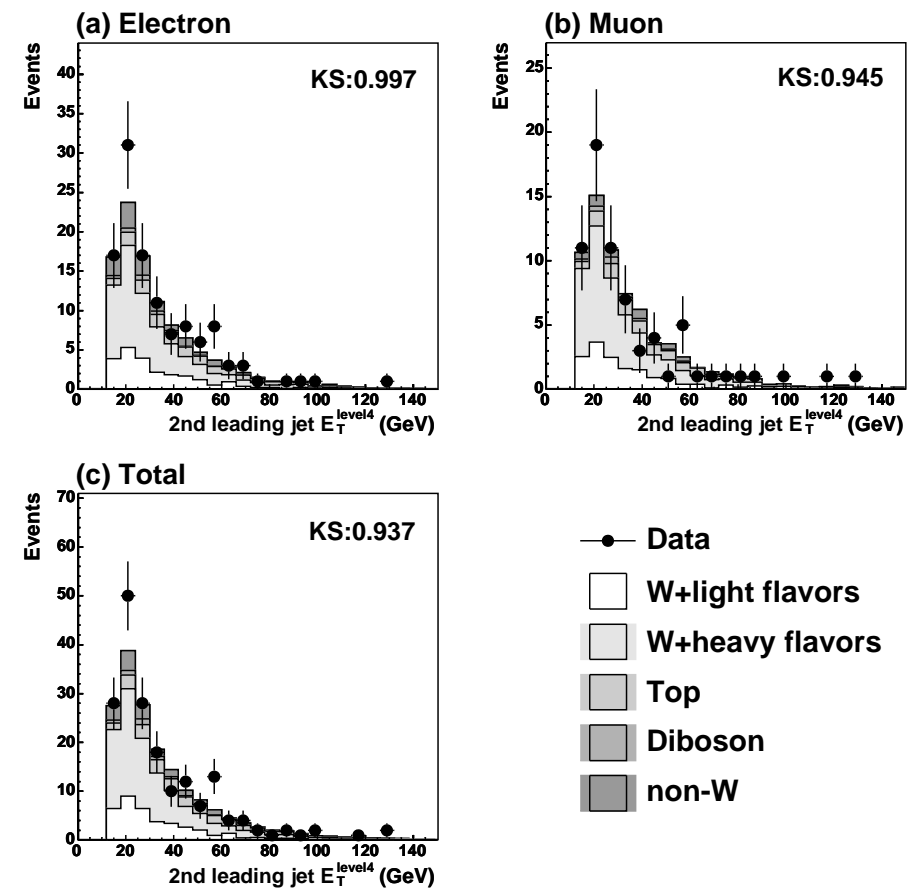

Figure 7.21: The 2nd leading jet $E_{T}$ distributions for $W+2$ jets tagged sample. (a)electron channel, (b)muon channel, (c)electron + muon channel. 

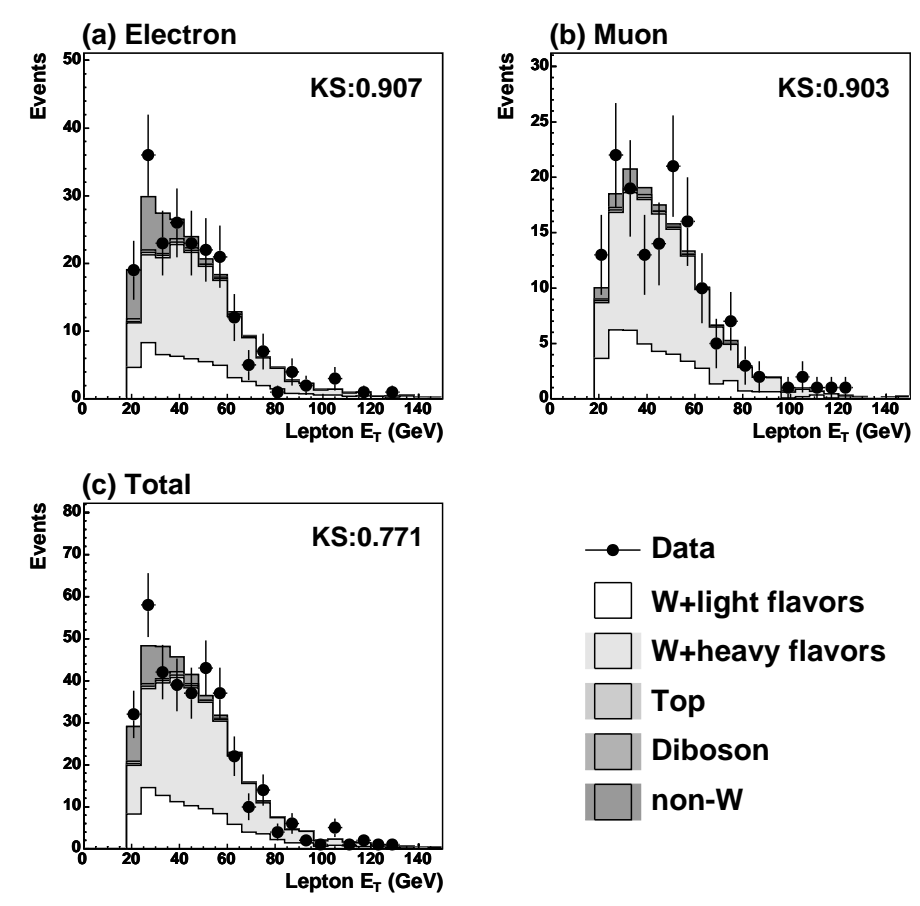

Figure 7.22: The lepton $E_{T}$ distributions for $W+1$ jet tagged sample. (a)electron channel, (b)muon channel, (c)electron + muon channel.
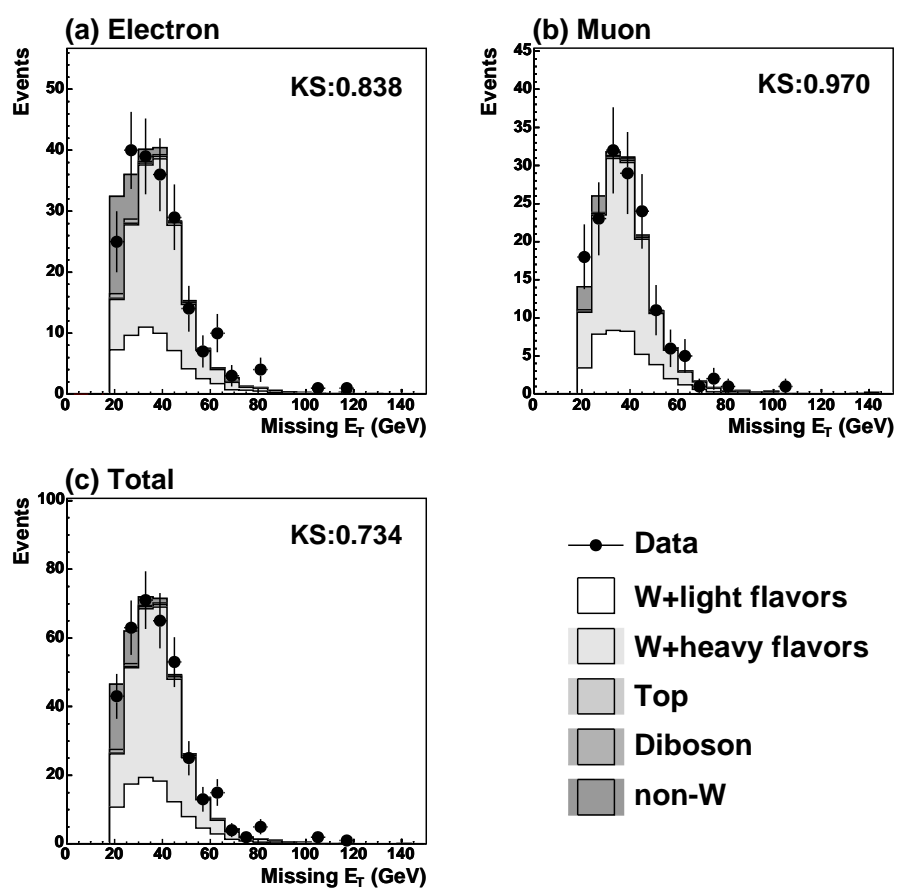

Figure 7.23: The $\mathscr{E}_{T}$ distributions for $W+1$ jet tagged sample. (a)electron channel, (b)muon channel, (c)electron + muon channel. 

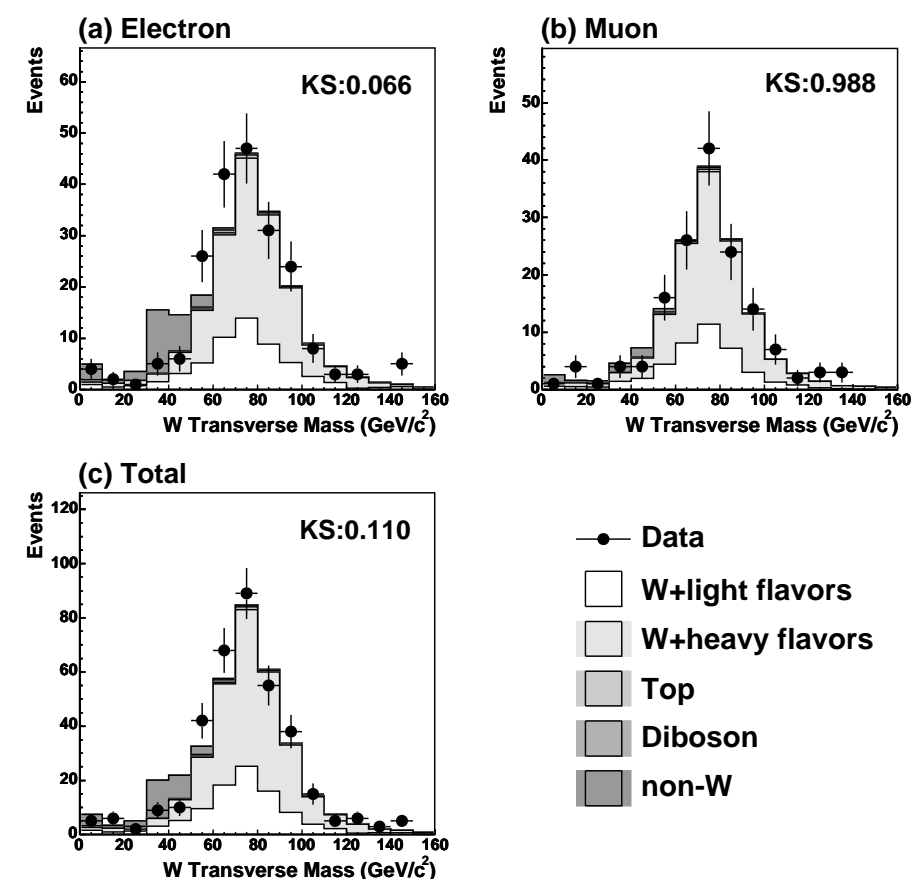

Figure 7.24: The $W$ transverse mass distributions for $W+1$ jet tagged sample. (a)electron channel, (b)muon channel, (c)electron + muon channel.

(a) Electron

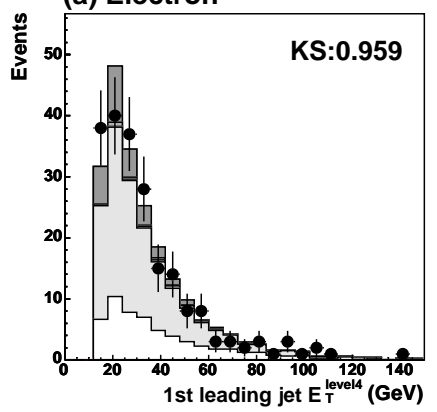

(c) Total

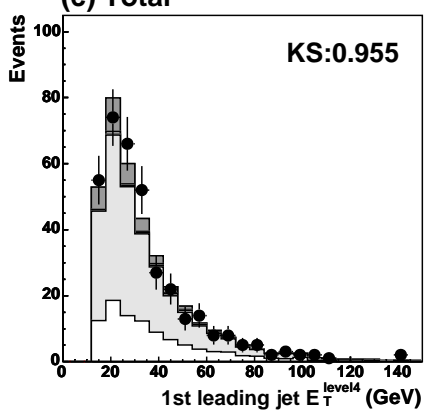

(b) Muon

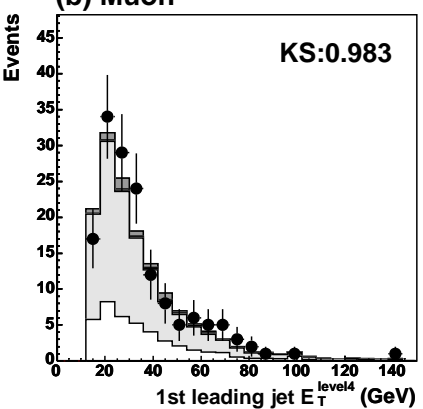

- Data

W+light flavors

W+heavy flavors

Top

Diboson

non-W

Figure 7.25: The 1st leading jet $E_{T}$ distributions for $W+1$ jet tagged sample. (a)electron channel, (b)muon channel, (c)electron + muon channel. 


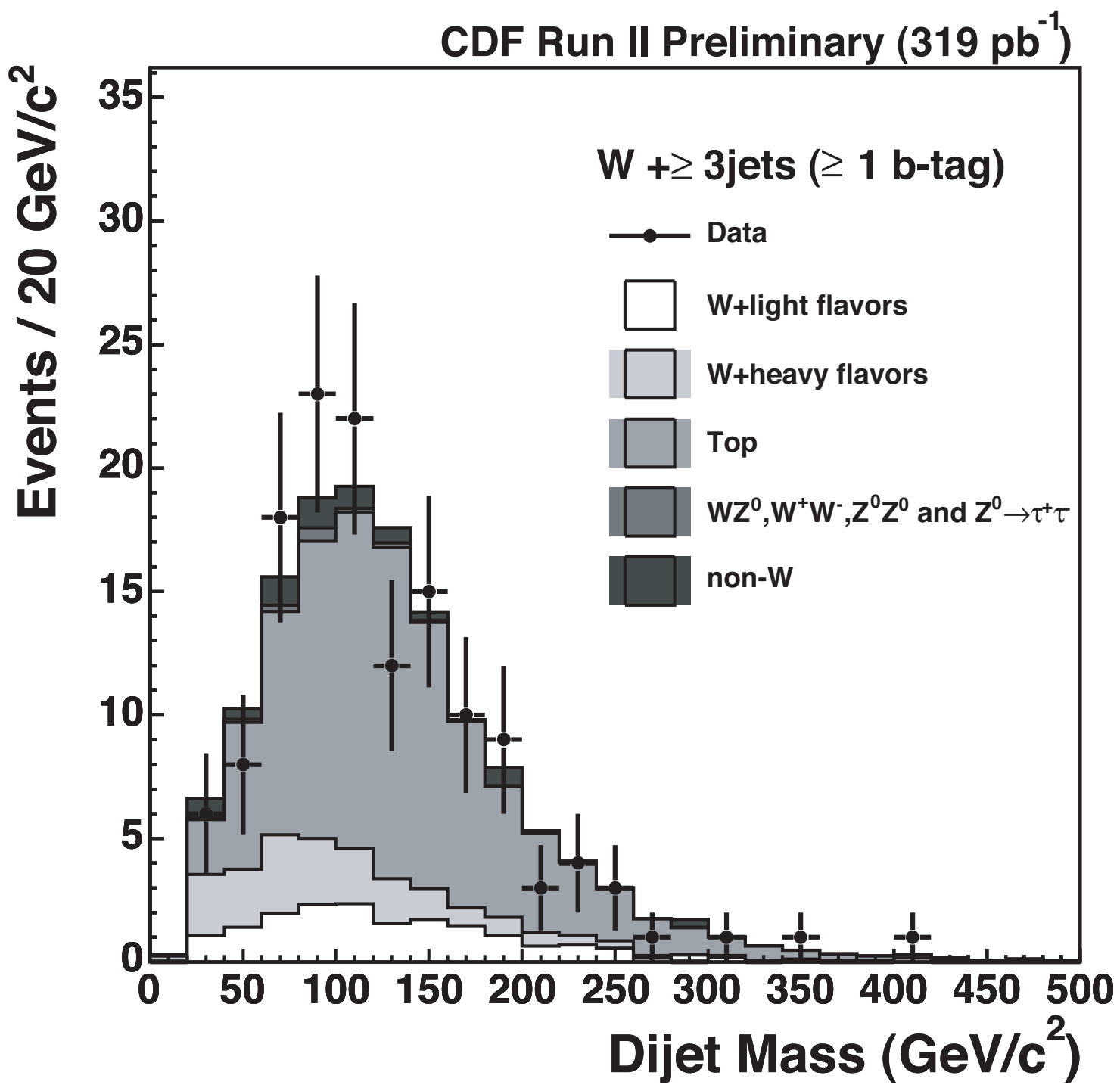

Figure 7.26: The invariant mass of the two leading jets in the $b$-tagged $W+\geq 3$ jets sample from data and expected backgrounds. 


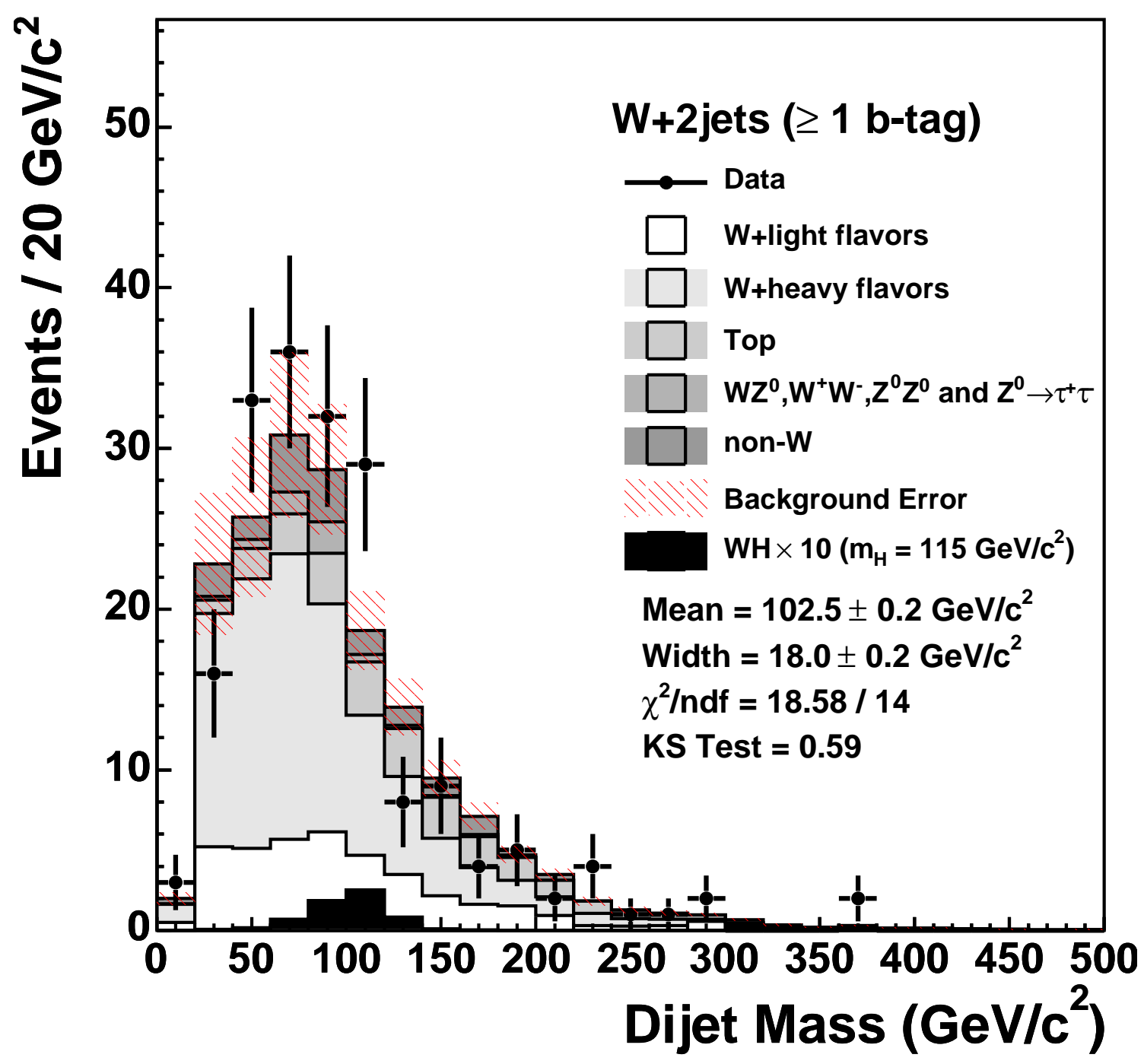

Figure 7.27: The dijet mass distribution in the data along with background samples. The Higgs boson mass distribution is scaled up by a factor of 10, where the Higgs boson mass is assumed to be $115 \mathrm{GeV} / c^{2}$. 

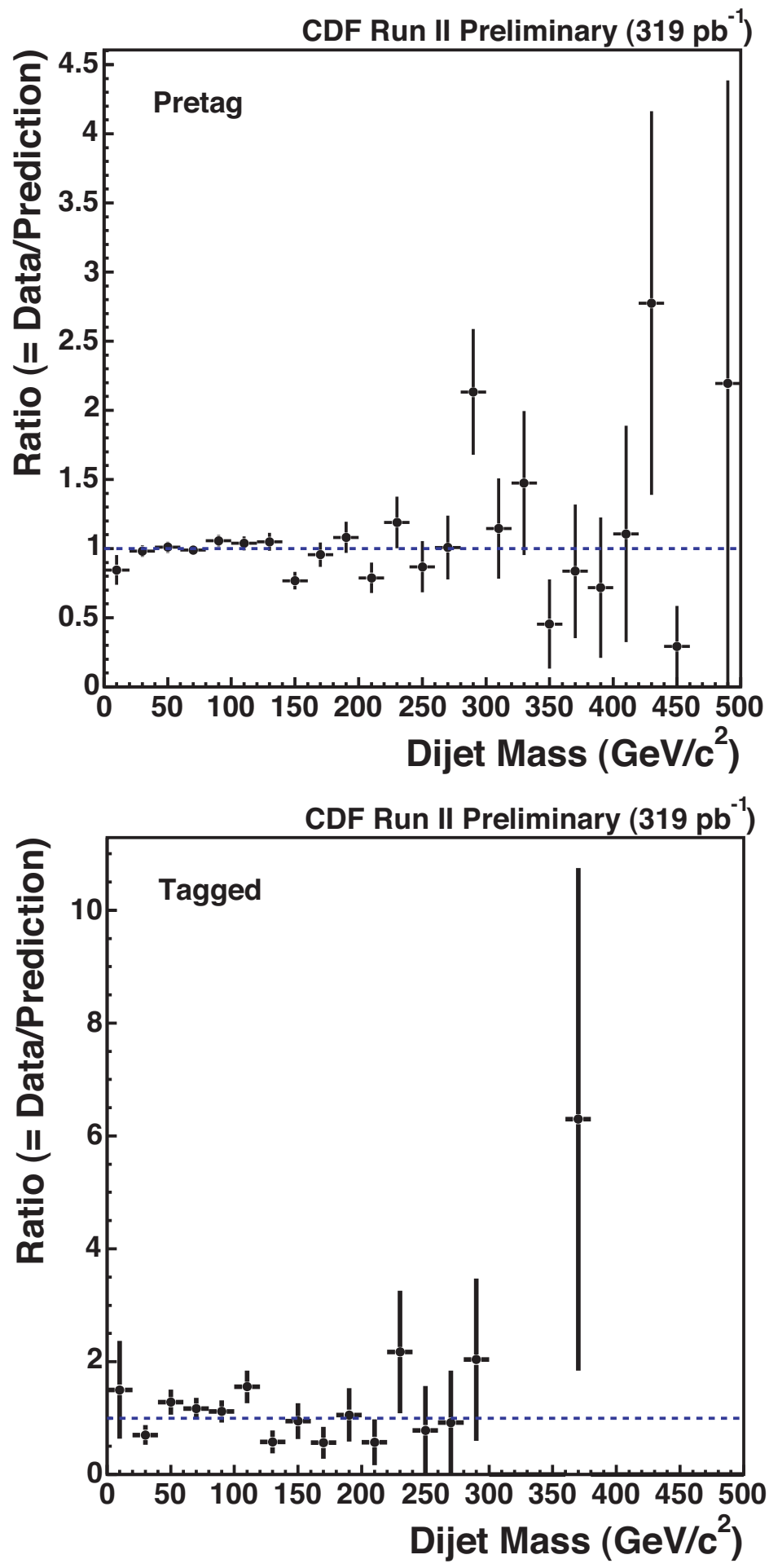

Figure 7.28: The ratio of the numbers of events between data and background expectation as a function of the dijet mass. (Top)pretag sample, (Bottom)tagged sample. 


\section{Chapter 8}

\section{Upper Limit on the Cross Section}

This chapter describes the final results of the Higgs boson search and methods used in obtaining the results. In the absence of a significant excess in the observed data w.r.t. the predicted background in the dijet invariant mass distribution as described in Chapter 7, a 95\% confidence level upper limit can be placed on the production cross section times branching ratio decaying into $b \bar{b}, \sigma(p \bar{p} \rightarrow W H) \times \mathcal{B} r(H \rightarrow b \bar{b})$, as a function of the Higgs boson mass.

\subsection{Procedure for Setting Limit}

To set the upper limits on $\sigma(p \bar{p} \rightarrow W H) \times \mathcal{B} r(H \rightarrow b \bar{b})$ at a $95 \%$ confidence level (C.L.), where $\sigma(p \bar{p} \rightarrow W H)$ and $\mathcal{B} r(H \rightarrow b \bar{b})$ denote the production cross section of $p \bar{p} \rightarrow W H^{1}$ and the branching ratio of $H \rightarrow b \bar{b}$, a binned maximum likelihood method is used with the contents of the bins treated with Poisson statistics [65]. The probability associated with $i$-th bin is

$$
P_{i}\left(n_{i}, \mu_{i}\right)=\frac{\mu_{i}^{n_{i}} e^{-\mu_{i}}}{n_{i} !}
$$

where $n_{i}$ is the number of events observed in $i$-th bin, $\mu_{i}$ is the number of events (signal + background) expected in the $i$-th bin. Then the likelihood function is defined as the total probability, which is the product of the individual probabilities:

$$
L(\alpha)=\prod_{i=1}^{N} P_{i}\left(n_{i}, \mu_{i}\right)=\prod_{i=1}^{N} \frac{\mu_{i}^{n_{i}} e^{-\mu_{i}}}{n_{i} !}
$$

where $N$ is the total number of bins. The $95 \%$ C.L. upper limit $\left(\alpha_{95}\right)$ is calculated by

$$
0.95=\frac{\int_{0}^{\alpha_{95}} L(\alpha) d \alpha}{\int_{0}^{\infty} L(\alpha) d \alpha}
$$

\footnotetext{
${ }^{1} \sigma(p \bar{p} \rightarrow W H)$ means the sum of $\sigma\left(p \bar{p} \rightarrow W^{+} H\right)$ and $\sigma\left(p \bar{p} \rightarrow W^{-} H\right)$.
} 
We note that in the limit of infinite statistics, the likelihood function becomes a Gaussian as a consequence of the central limit theorem. In this case, Equation 8.2 becomes equivalent to

$$
\ln L=\ln L_{\max }-\frac{s^{2}}{2}
$$

where $s$ is the number of standard deviations. For 95\% C.L., one finds $s=1.96$. The corresponding limit value then follows

$$
\ln L_{\max }-\ln L=1.92 \text {. }
$$

Figure 8.1 shows the logarithm of a likelihood function compared to the likelihood function itself. This illustrates the two different methods which can be used to derive the $95 \%$ C.L. on the parameter $\alpha$. Generally, both methods of maximum likelihood and likelihood integration yield the same limits. However, we use likelihood integration for setting the $95 \%$ C.L. upper limit.

The systematic uncertainty, $\Delta \alpha$, must be incorporated into the likelihood function. We convolute the systematic uncertainty into the likelihood function by smearing each point of $L$ by a Gaussian distribution centered at that point with width $\Delta \alpha:$

$$
L_{\text {smear }}(\alpha)=\int_{0}^{\infty} L\left(\alpha^{\prime}\right) \frac{e^{-\left(\alpha-\alpha^{\prime}\right)^{2} / 2 \Delta \alpha^{\prime 2}}}{\sqrt{2 \pi \Delta \alpha^{\prime 2}}} d \alpha^{\prime} .
$$

\subsection{Signal Acceptance}

The expected number of signal events, $N_{W H \rightarrow \ell \nu b \bar{b}}$, is calculated from the following equation:

$$
N_{W H \rightarrow \ell \nu b \bar{b}}=\varepsilon_{W H \rightarrow \ell \nu b \bar{b}} \cdot \mathcal{L} \cdot \sigma(p \bar{p} \rightarrow W H) \cdot \mathcal{B} r(H \rightarrow b \bar{b}),
$$

where $\varepsilon_{W H \rightarrow \ell \nu b \bar{b}}$ and $\mathcal{L}$ are the signal acceptance and the luminosity. The acceptance is split into various efficiencies and correction factors as follows:

$$
\varepsilon_{W H \rightarrow \ell \nu b \bar{b}}=\varepsilon_{z_{0}} \cdot \varepsilon_{\text {trig }} \cdot \varepsilon_{W H \rightarrow \ell^{\prime} \nu b \bar{b}}^{\mathrm{MC}} \cdot\left(\sum_{\ell^{\prime}=e, \mu, \tau} \mathcal{B} r\left(W \rightarrow \ell^{\prime} \nu\right)\right) \cdot s_{\text {lepton }},
$$

where $\varepsilon_{z_{0}}$ is the efficiency of the $\left|z_{0}\right|<60 \mathrm{~cm}$ cut, $\varepsilon_{\text {trig }}$ is the trigger efficiency for identifying a single high- $p_{T}$ lepton, and $\varepsilon_{W H \rightarrow \ell^{\prime} \nu b \bar{b}}^{\mathrm{MC}}$ is the fraction of $W H \rightarrow \ell^{\prime} \nu b \bar{b}$ $\left(\ell^{\prime}=e, \mu, \tau\right) \mathrm{MC}$ events which pass all the selection cuts including the reconstruction efficiency, as well as the efficiencies of the kinematic cuts and of the $b$-tagging algorithm. $\mathcal{B} r\left(W \rightarrow \ell^{\prime} \nu\right)$ and $s_{\text {lepton }}$ are the branching ratio of $W \rightarrow \ell^{\prime} \nu$ and a factor that corrects for the lepton identification efficiency difference between data and MC.

Samples of Pүтніа $W H \rightarrow \ell^{\prime} \nu b \bar{b}$ MC with the Higgs boson masses of $m_{H}=110$, 115, 120, 130, 140 and $150 \mathrm{GeV} / c^{2}$ are used to estimate $\varepsilon_{W H \rightarrow \ell^{\prime} \nu b \bar{b}}^{M C}$. Table 8.1 and Figure 8.2 show the definition of event selection numbers and the selection efficiency for $m_{H}=115 \mathrm{GeV} / c^{2}$ using the $W H \rightarrow \ell^{\prime} \nu b \bar{b}$ MC sample in the $W+2$ jets bin.

The $\mathrm{MC}$ acceptance is corrected for the $b$-tagging efficiency, and other three efficiencies using data and MC: 

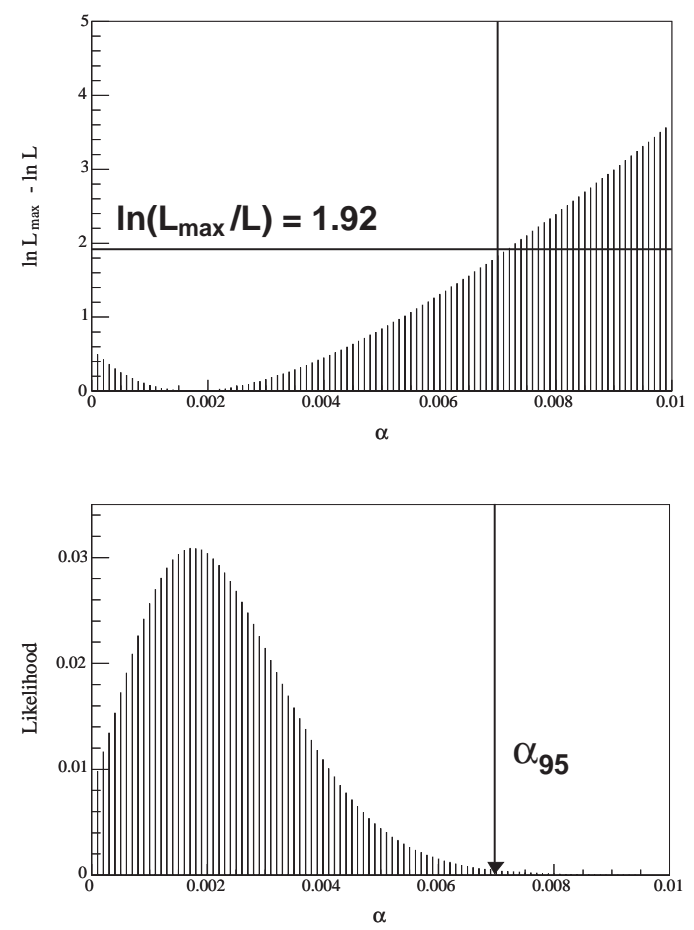

Figure 8.1: The logarithm of a likelihood function (top) compared to the likelihood function itself (bottom), illustrating the two different methods which can be used to derive the $95 \%$ C.L. on the parameter $\alpha$.

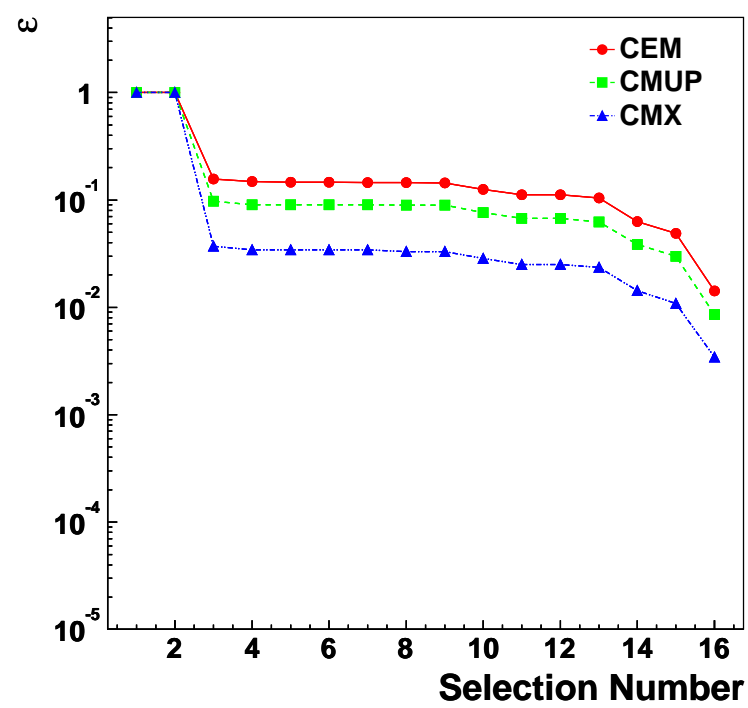

Figure 8.2: The event selection efficiency for $m_{H}=115 \mathrm{GeV} / c^{2}$ using the $W H \rightarrow$ $\ell^{\prime} \nu b \bar{b}\left(\ell^{\prime}=e, \mu, \tau\right) \mathrm{MC}$ sample in the $W+2 \mathrm{jets}$ bin. The selection number is described in Table 8.1. 


\begin{tabular}{cl}
\hline \hline Selection Number & Event Selection \\
\hline 1 & Initial (After good run requirement) \\
2 & Trigger Requirement \\
3 & Lepton ID \\
4 & Lepton Isolation \\
5 & Conversion or Cosmic Veto \\
6 & $\left|z_{0}-z_{\text {lepton }}\right|<5.0 \mathrm{~cm}$ \\
7 & Isolated Track Veto \\
8 & Dilepton Veto \\
9 & $Z^{0}$ Veto \\
10 & $\mathbb{E}_{T}>20 \mathrm{GeV}$ \\
11 & Extra Jet Veto \\
12 & $E_{T}^{\text {jet1 }}>15 \mathrm{GeV}$ (No effect) \\
13 & At Least One Taggable Jet \\
14 & At Least One Tagged Jet \\
15 & Single Tag \\
16 & Double Tags \\
\hline \hline
\end{tabular}

Table 8.1: The definition of event selection numbers.

- The $b$-tagging efficiency is corrected for the $b$-tagging scale factor of $\varepsilon_{\text {data }} / \varepsilon_{\mathrm{MC}}=$ $0.909 \pm 0.060[51,52,53]$ by randomly keeping only $90.9 \%$ of all the tags, and discarding the others.

- The efficiency of the $z_{0}$ cut is measured from data: $\varepsilon_{z_{0}}=0.951 \pm 0.003$ [66].

- The trigger efficiency $\varepsilon_{\text {trig }}$ is measured from each independent trigger. It is different for each type of lepton: $\varepsilon_{\text {trig }}^{\mathrm{CEM}}=0.962 \pm 0.007$ [67], $\varepsilon_{\text {trig }}^{\mathrm{CMUP}}=0.908 \pm$ 0.005 and $\varepsilon_{\text {trig }}^{\mathrm{CMX}}=0.965 \pm 0.004[68]$.

- The scale factor $s_{\text {lepton }}$ is evaluated by comparing a data sample of $Z^{0}$ events with a PYTHIA $Z^{0}$ sample, and found to be $s_{\text {lepton }}^{\text {CEM }}=0.996 \pm 0.052$ [69], $s_{\text {lepton }}^{\text {CMUP }}=$ $0.822 \pm 0.048$ and $s_{\text {lepton }}^{\mathrm{CMX}}=0.976 \pm 0.050[68]$.

Systematic uncertainties on the acceptance are:

- Lepton isolation:

The scale factor $s_{\text {lepton }}$ is evaluated in a $Z^{0}$ sample with little jet activity, while this analysis uses two jets events, so that leptons tend to be less isolated in this analysis. The statistically limited $Z^{0}+$ jet sample is used to check the isolation dependence on $s_{\text {lepton }}$ and a $5 \%$ systematic uncertainty is assigned [70].

- Jet energy scale:

Based on the single jet energy uncertainty, the jet energies in the $W H \mathrm{MC}$ 
samples are shifted by $\pm 1 \sigma$. The difference from the nominal acceptance is taken as the systematic uncertainty $(3 \%)$.

- Secondary vertex $b$-tagging:

Based on the $b$-tagging scale factor uncertainty as described in Section 5.5, the scale factor in the $W H$ MC sample is shifted by $\pm 1 \sigma$. The difference from the nominal acceptance is taken as the systematic uncertainty (5\%).

- Initial and final state radiation (ISR and FSR):

In PYTHIA, the parameters related to ISR and FSR are varied by half and double of PYTHIA default values. The difference from the nominal acceptance is taken as the systematic uncertainty $(3 \%$ and $7 \%)$.

- Parton distribution function (PDF) [36, 71]:

The uncertainty in the distribution of the proton (antiproton) momentum among its constituent partons affects the momentum of the $W H$ system. In the CTEQ parametrization, PDFs are described by 20 independent eigenvectors. In next to the leading order (NLO) version of PDFs, CTEQ6M, a $90 \%$ confidence interval is provided for each eigenvector. Using the $\pm 90 \%$ value of each eigenvector, we compute a new acceptance by reweighting our default CTEQ5L PYTHIA $W H$ sample. We add in quadrature the differences between the weighted acceptances for the 20 eigenvectors w.r.t. the weighted acceptance from the nominal CTEQ6M. The difference between the leading order CTEQ5L and the next to leading order CTEQ6M PDFs, as well as the uncertainty on $\alpha_{s}$ (QCD coupling constant) are added in quadrature, resulting in a total uncertainty of $1 \%$.

- Jet energy smearing:

The studies of $\gamma+$ jet balancing indicate that the jet energy resolution in PYTHIA is better than in the data. We have estimated the difference in the acceptance after smearing the jet energy in MC with additional $10 \%$ of jet energy resolution. The difference is small about $1 \%$ for the uncertainty.

- Soft jet modeling:

Although we used extra jet veto in the offline event selections, described in Section 4.6.2, the soft jet may not be simulated well in MC. We have used the inclusive $W+0$ jet and $W+1$ jet data and MC samples to estimate the soft $E_{T}$ jet rejection factors. Figure 8.3 shows the soft $E_{T}$ jet selection efficiencies. We take the difference (1\%) as the systematic uncertainty for soft jet modeling.

Table 8.2 summarizes the systematic uncertainties. Figure 8.4 shows the overall acceptance including all systematic effects as a function of $m_{H}$. 


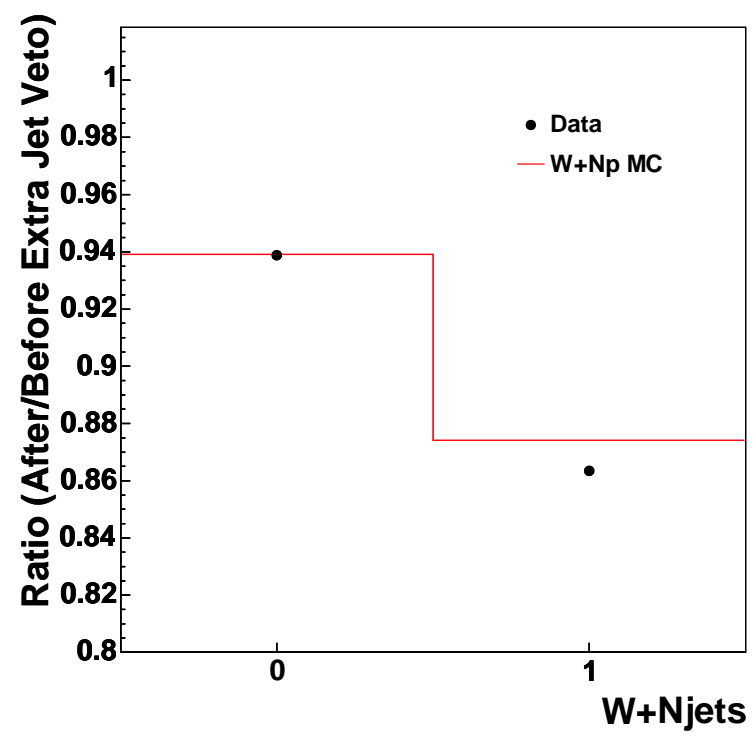

Figure 8.3: The extra jet selection efficiency for data and MC.

\begin{tabular}{cc}
\hline \hline source & uncertainty (\%) \\
\hline Lepton ID & 5 \\
Trigger & $<1$ \\
PDF & 1 \\
ISR & 3 \\
FSR & 7 \\
Jet energy scale & 3 \\
Secondary vertex tag & 5 \\
Jet energy smearing & 1 \\
Soft jet modeling & 1 \\
\hline Total & 11 \\
\hline \hline
\end{tabular}

Table 8.2: Systematic uncertainties on the signal acceptance. 


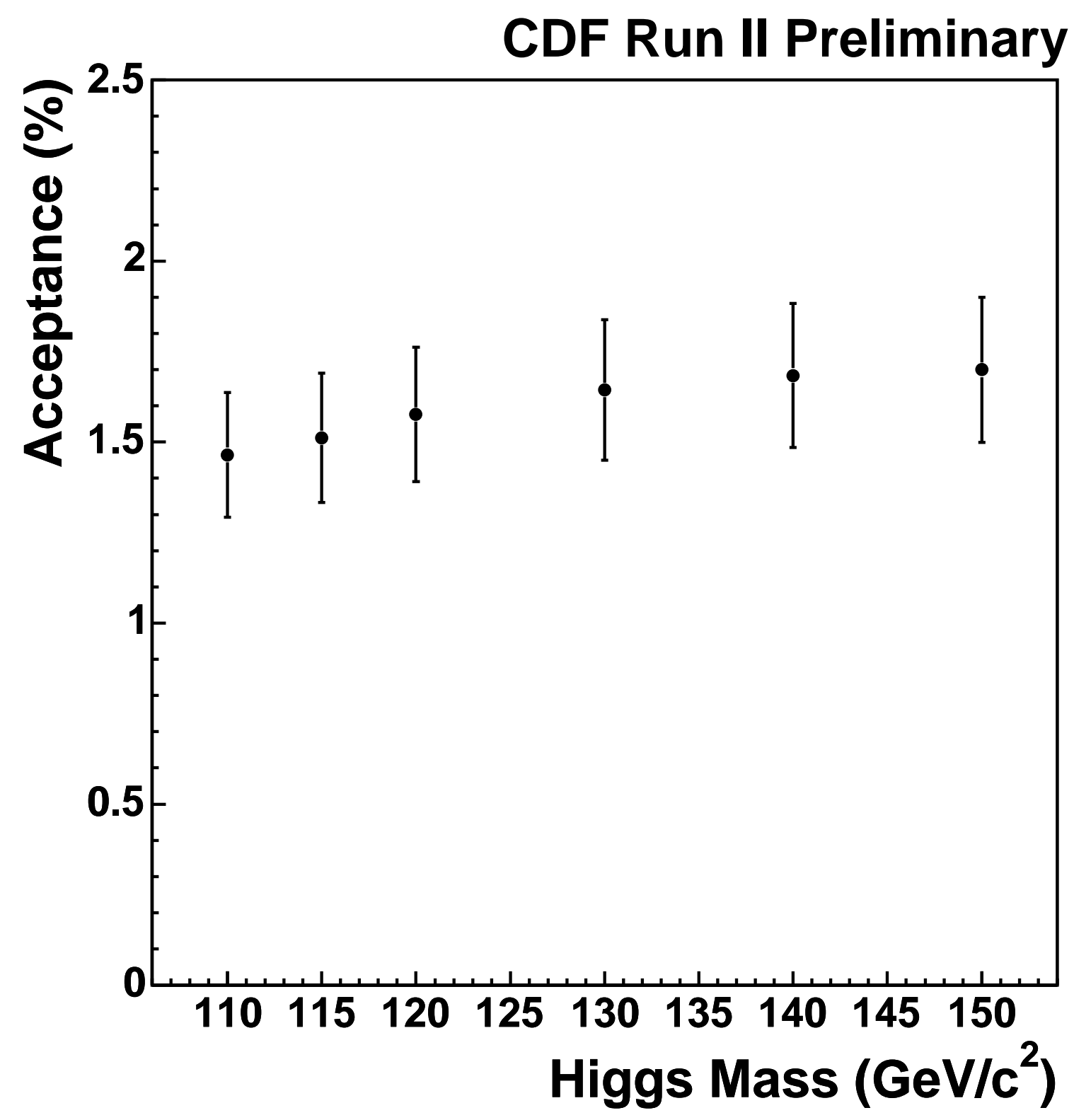

Figure 8.4: The overall $W H \rightarrow W b \bar{b}$ acceptance with overall systematic uncertainty (11\%) as a function of $m_{H}$. 


\subsection{A 95\% Confidence Level Limit on the Higgs Boson Production}

In the absence of a significant excess in the observed data w.r.t. the predicted background in the dijet invariant mass distribution, a 95\% C.L. upper limit can be placed on the production cross section times branching ratio decaying into $b \bar{b}$, $\sigma(p \bar{p} \rightarrow W H) \times \mathcal{B} r(H \rightarrow b \bar{b})$, as a function of the Higgs boson mass.

We assume the dijet mass distribution in the data consists of QCD (mistags, $W+b \bar{b}, W+c \bar{c}, W+c$ and diboson), TOP ( $t \bar{t}$ and single top) and $W H$ events. A binned maximum likelihood technique, described in Section 8.1, is used to estimate the number of $W H$ signal events by constraining the number of QCD and TOP events to the expectation within the statistical and systematic uncertainties. The expected number of events $(\mu)$ in each mass bin is

$\mu=f_{\mathrm{QCD}} \cdot N_{\mathrm{QCD}}+f_{\mathrm{TOP}} \cdot N_{\mathrm{TOP}}+f_{W H} \cdot\left(\varepsilon_{W H \rightarrow W b \bar{b}} \cdot \mathcal{L} \cdot \sigma(p \bar{p} \rightarrow W H) \cdot \mathcal{B} r(H \rightarrow b \bar{b})\right)$,

where $f_{\mathrm{QCD}}, f_{\mathrm{TOP}}$ and $f_{W H}$ are the expected fraction of events in a given mass bin predicted by MC. $N_{\mathrm{QCD}}$ and $N_{\mathrm{TOP}}$ are the expected number of QCD and TOP events, respectively.

The corresponding likelihood is

$$
L=\prod_{i=\text { bin }} \frac{\mu_{i}^{N_{i}} \cdot e^{-\mu_{i}}}{N_{i} !} \times G\left(N_{\mathrm{QCD}}, \sigma_{N_{\mathrm{QCD}}}\right) \times G\left(N_{\mathrm{TOP}}, \sigma_{N_{\mathrm{TOP}}}\right),
$$

where $N_{i}$ is the observed number of events from $W+2$ jets sample and $G$ is a Gaussian constraint on the estimates of QCD and TOP background events. Then we fit the dijet mass distribution with this likelihood function. Figure 8.5 and Figure 8.6 show the $-\ln \mathcal{L}$ and likelihood distributions as a function of the cross section. Figure 8.7 shows the $95 \%$ C.L. upper limits as a function of $m_{H}$ with the expected limits from pseudo-experiments which are described in Section 8.4 [14, 15]. Table 8.3 shows the cross section fit values with errors and the upper limits. Since there is some events remaining in the region from 40 to $120 \mathrm{GeV} / c^{2}$, the measurement gives a worse limit than the expectation. However, it is consistent with the expected limits. Although we have improved the limit compared to Run 1, the sensitivity of the present search is limited by statistics. The observed limit is approximately one or two orders of magnitude higher than the predicted cross section for Standard Model Higgs boson production.

\subsection{Pseudo-Experiments}

The pseudo-experiment is a mock numeric test. Since we have the number of expected backgrounds and the signal acceptances for the $b$-tagged $W+2$ jets events, we can simulate the number of observed events and also the $95 \%$ C.L. upper limits on the $W H$ production cross section. To validate the measured upper limits, we have made pseudo-experiments to check whether the measured upper limits are 


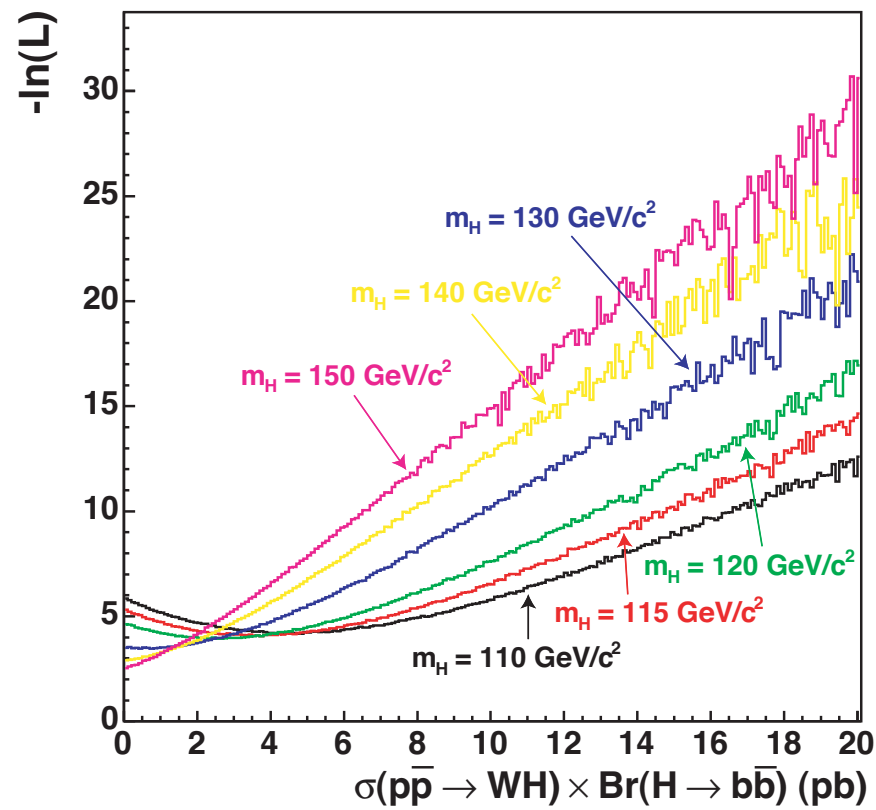

Figure 8.5: The $-\ln \mathcal{L}$ distributions as a function of the cross section for the dijet mass fit.

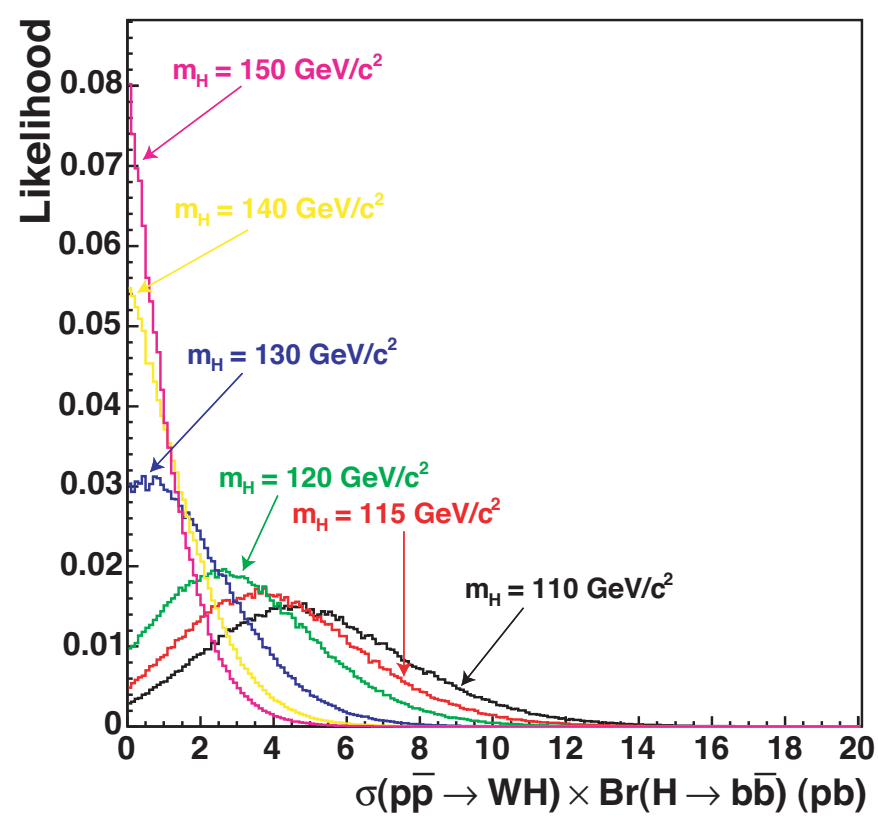

Figure 8.6: The likelihood distributions as a function of the cross section for the dijet mass fit. 


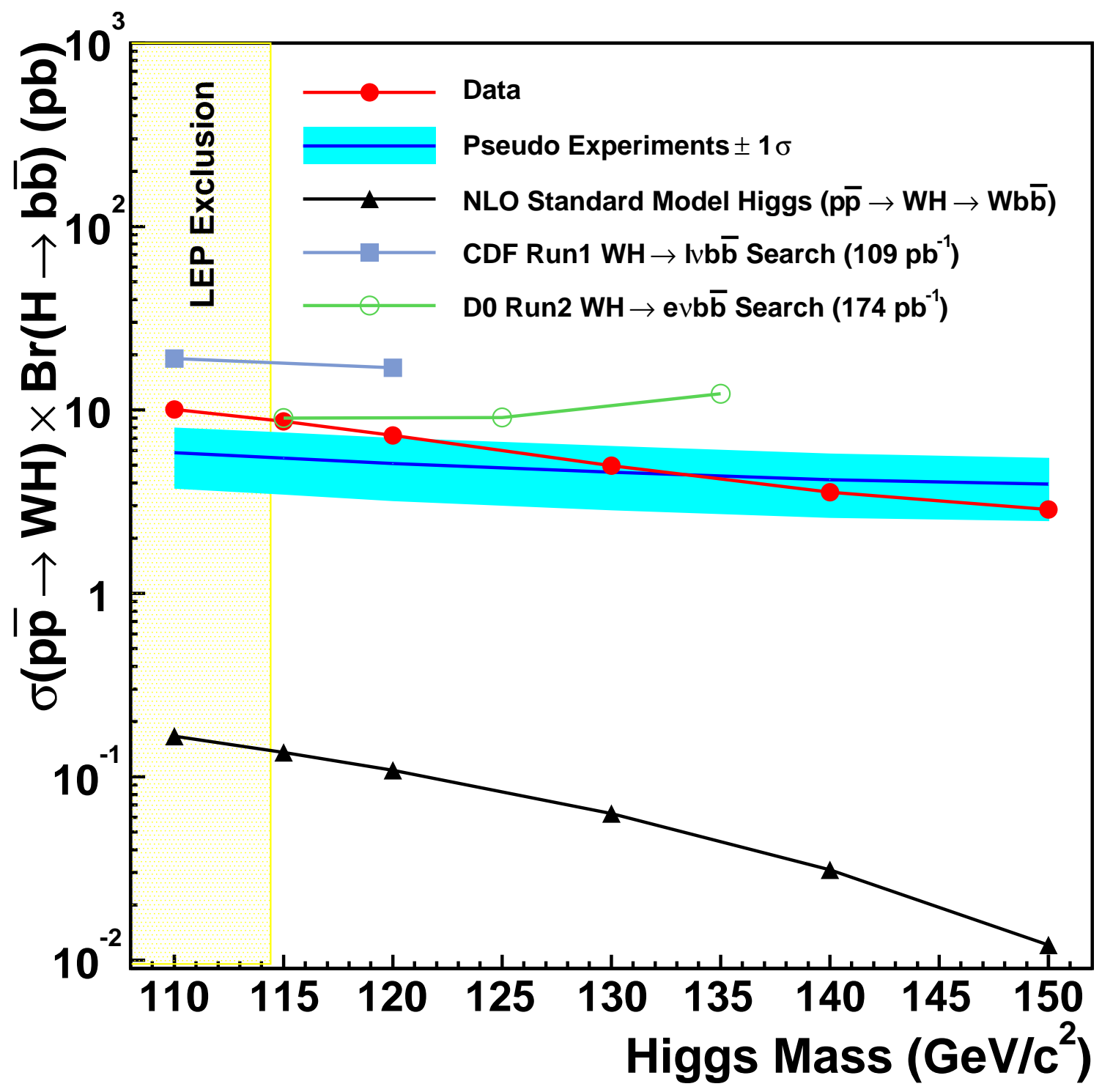

Figure 8.7: The 95\% C.L. upper limits (red circles) on the $W H$ cross section as a function of the Higgs boson mass. Also shown are the theoretical cross section (black triangles) for Standard Model Higgs boson production in association with a $W$ boson and the expected limit results from pseudo-experiments (blue region). 


\begin{tabular}{ccc}
\hline \hline$m_{H}$ & Fit Value $(\mathrm{pb})$ & Upper Limit $(\mathrm{pb})$ \\
\hline 110 & $4.8_{-2.9}^{+2.7}$ & 10.0 \\
115 & $3.7_{-2.5}^{+2.3}$ & 8.6 \\
120 & $2.6_{-2.3}^{+2.3}$ & 7.2 \\
130 & $0.4_{-0.0}^{+2.1}$ & 4.9 \\
140 & $0.0_{-0.0}^{+1.2}$ & 3.5 \\
150 & $0.0_{-0.0}^{+0.8}$ & 2.8 \\
\hline \hline
\end{tabular}

Table 8.3: The fit values with errors and the $95 \%$ C.L. upper limits.

reasonable by checking the statistical fluctuations expected from the background expectations and the signal acceptances. We make 1000 pseudo-experiments by fluctuating the background and the acceptance by Gaussians. Figure 8.8 shows the 95\% C.L. upper limit distributions from the pseudo-experiments. In the figure, the "prob." means probability to measure the upper limit from data. The measured upper limits from pseudo-experiments are consistent with the observed limits.

\subsection{Double $b$-Tagging as an Cross Check}

We have examined the double $b$-tagging requirements to check the excess of events between 40 and $120 \mathrm{GeV} / c^{2}$ :

- $\geq 1$ tag (At least one $b$-tagging method),

- $\geq 2$ tags (Double $b$-tagging method).

We require one of the jets to be tagged by SecVtx and the second jet to be also tagged by SecVtx. Table 8.4 and Figure 8.9 show the observed and estimated number of events as a function of jet multiplicity. Figure 8.10 shows the reconstructed dijet mass distributions. The observed number of events and dijet mass distribution are consistent with the estimated number of events and dijet mass distribution.

At first, we compare the significances between at least one $b$-tagging method and double $b$-tagging method. Figure 8.11 shows the significance $(S / \sqrt{B})$ comparison as a function of $m_{H}$. Table 8.5 shows the numbers of signal and background for $m_{H}=$ $115 \mathrm{GeV} / c^{2}$. The double tags method does not seem to improve the significance.

Table 8.6 and Figure 8.12 show the signal acceptance, and Figure 8.13 shows the $95 \%$ C.L. upper limits as a function of $m_{H}$ for double tags. The double $b$-tagging method does not improve the upper limits over at least one $b$-tagging method.

\subsection{Other Direct Standard Model Higgs Boson Searches at Tevatron}

The CDF and D $\varnothing$ collaborations are actively searching for the Higgs boson by making use of a variety of search channels. Figure 8.14 shows the compilation 

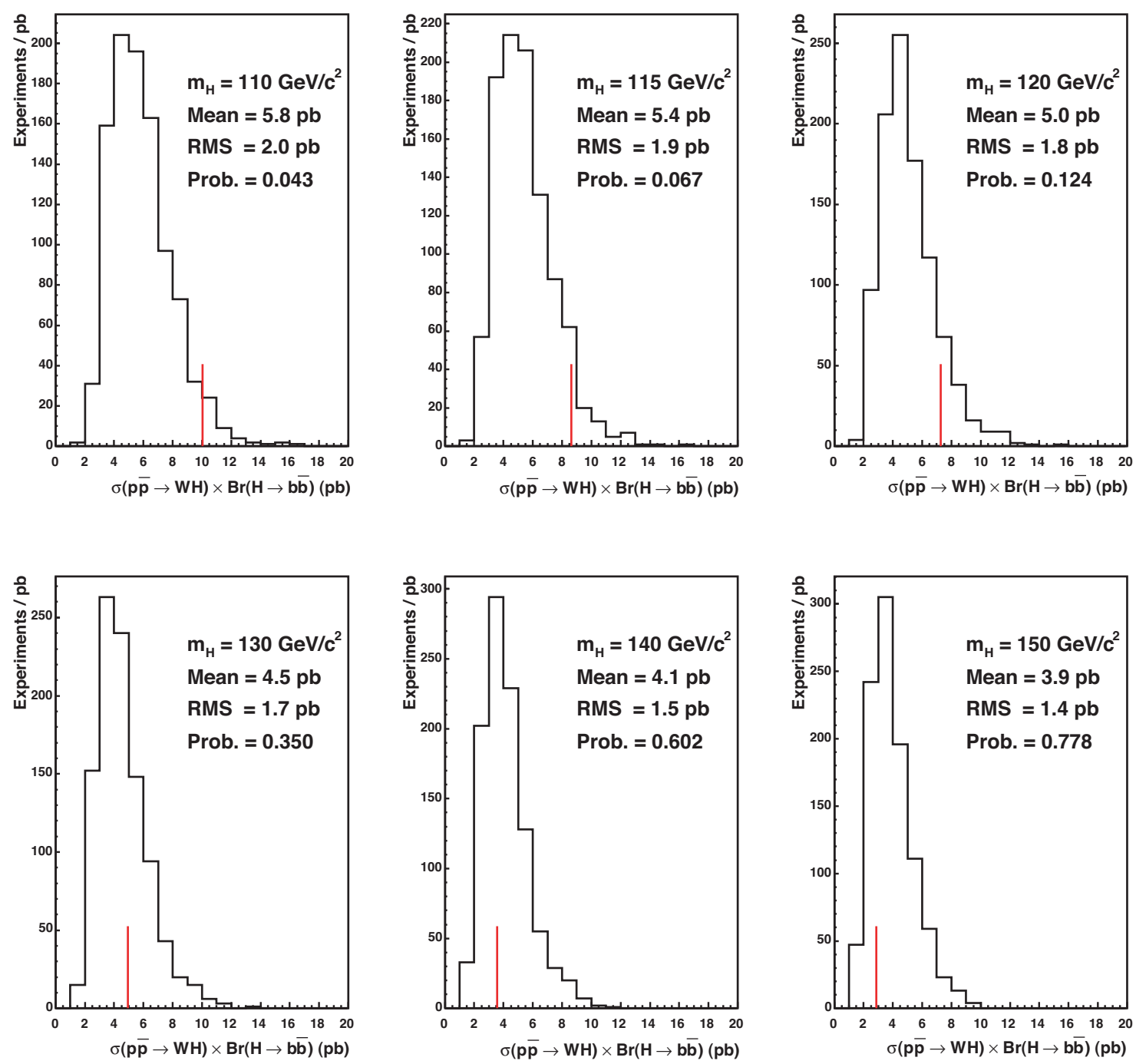

Figure 8.8: Comparison of observed limits with the expected limits from pseudoexperiments. The red line shows the measured upper limits. (a) $m_{H}=110 \mathrm{GeV} / c^{2}$, (b) $m_{H}=115 \mathrm{GeV} / c^{2}$, (c) $m_{H}=120 \mathrm{GeV} / c^{2}$, (d) $m_{H}=130 \mathrm{GeV} / c^{2}$, (e) $m_{H}=140$ $\mathrm{GeV} / c^{2}$, (f) $m_{H}=150 \mathrm{GeV} / c^{2}$. The lines show the measured upper limits. In figure, the "prob." means probability to measure the upper limit from data. 


\begin{tabular}{cccc}
\hline \hline Background & $W+2$ jets & $W+3$ jets & $W+\geq 4$ jets \\
\hline Mistags & $1.03 \pm 0.11$ & $0.41 \pm 0.06$ & $0.21 \pm 0.03$ \\
$W+b \bar{b}$ & $8.04 \pm 2.96$ & $1.32 \pm 0.47$ & $0.25 \pm 0.12$ \\
$W+c \bar{c}$ & $0.41 \pm 0.15$ & $0.08 \pm 0.03$ & $0.02 \pm 0.01$ \\
non- $W$ & $0.38 \pm 0.13$ & $0.31 \pm 0.11$ & $0.12 \pm 0.04$ \\
Diboson $/ Z^{0} \rightarrow \tau^{+} \tau^{-}$ & $0.34 \pm 0.06$ & $0.10 \pm 0.03$ & $0.02 \pm 0.00$ \\
single top & $1.30 \pm 0.30$ & $0.43 \pm 0.12$ & $0.09 \pm 0.04$ \\
$t \bar{t}$ & $3.12 \pm 0.54$ & $8.31 \pm 1.45$ & $15.98 \pm 2.78$ \\
\hline Total Background & $14.62 \pm 3.25$ & $10.96 \pm 1.68$ & $16.69 \pm 2.84$ \\
\hline Observed positive tags & 14 & 12 & 19 \\
\hline \hline
\end{tabular}

Table 8.4: The number of observed double positive tagged events and the background summary for an integrated luminosity of $318.5 \mathrm{pb}^{-1}$ for CEM and CMUP and 305.2 $\mathrm{pb}^{-1}$ for CMX.
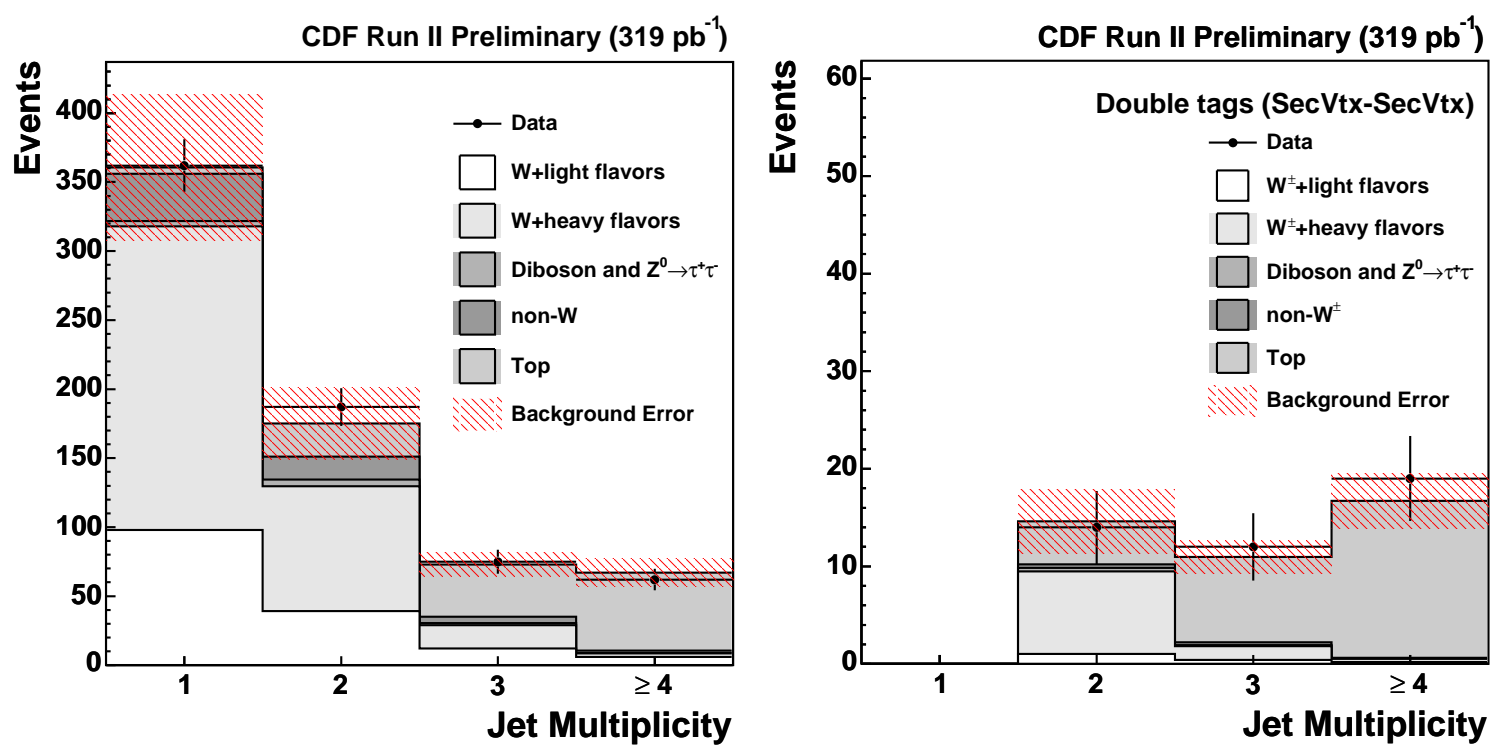

Figure 8.9: The observed and estimated number of events for (Left)at least one $b$-tagging method, (Right)double $b$-tagging method. The left plot is the same as Figure 6.2.

\begin{tabular}{ccc}
\hline \hline Methods & Signal & Background \\
\hline At least one $b$-tagging & 0.53 & 62.8 \\
Double $b$-tagging & 0.12 & 5.07 \\
\hline \hline
\end{tabular}

Table 8.5: The numbers of signal and background in $1.5 \sigma$ mass window for $m_{H}=115$ $\mathrm{GeV} / c^{2}$. 

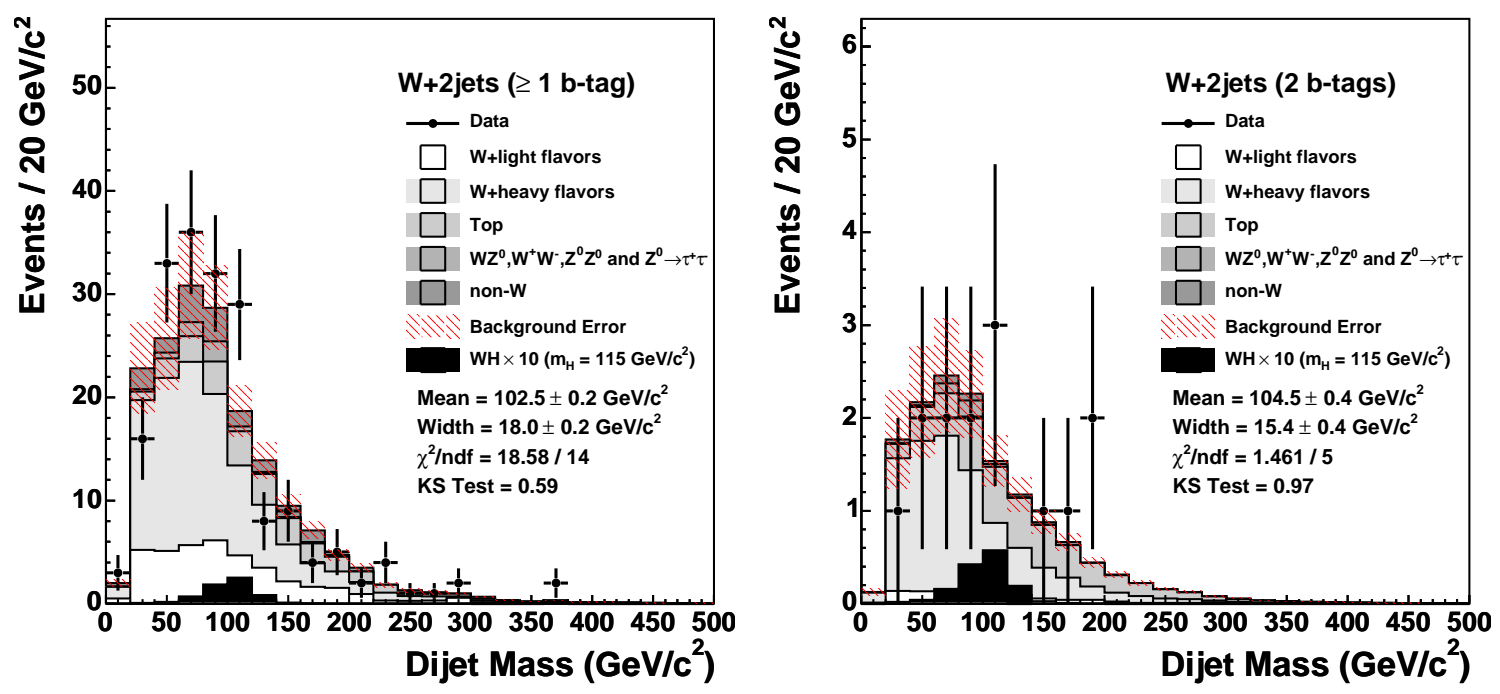

Figure 8.10: The dijet mass distributions for (Left)at least one $b$-tagging method, (Right)double $b$-tagging method. The left plot is the same as Figure 7.27.

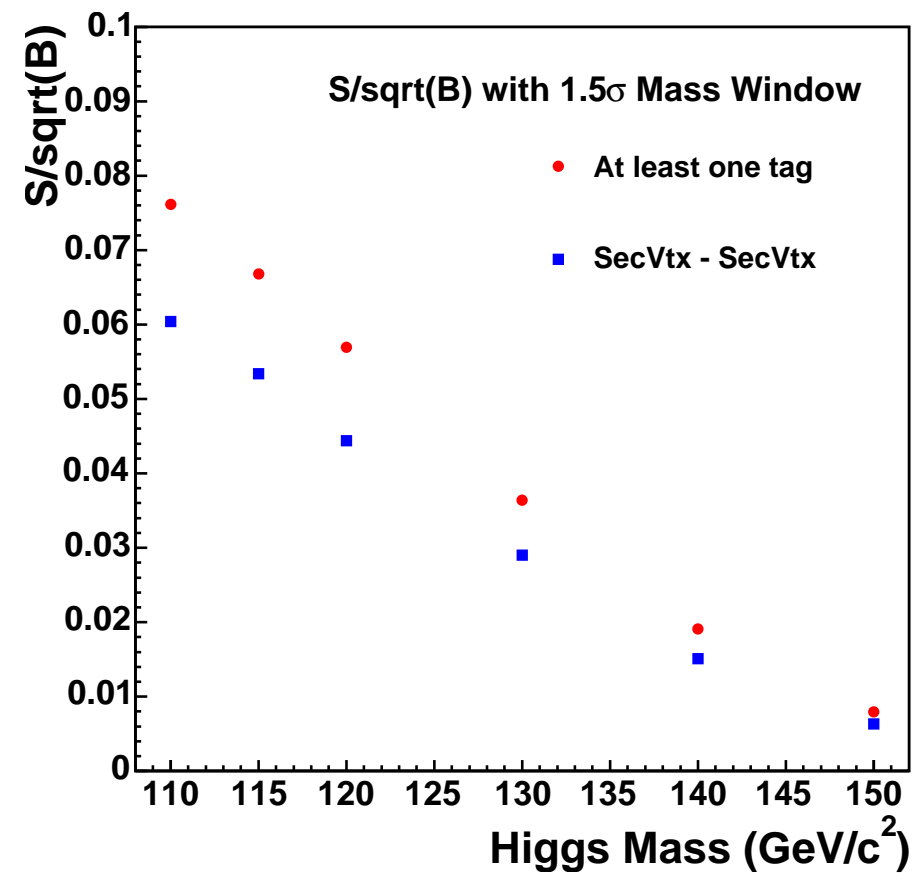

Figure 8.11: Significance as a function of Higgs mass for the selection with at least one $b$-tagging or with double $b$-tagging. 


\begin{tabular}{cc}
\hline \hline Methods & Acceptance $(\%)$ \\
\hline At least one $b$-tagging & 1.51 \\
Double $b$-tagging & 0.34 \\
\hline \hline
\end{tabular}

Table 8.6: The signal acceptance for $m_{H}=115 \mathrm{GeV} / c^{2}$.
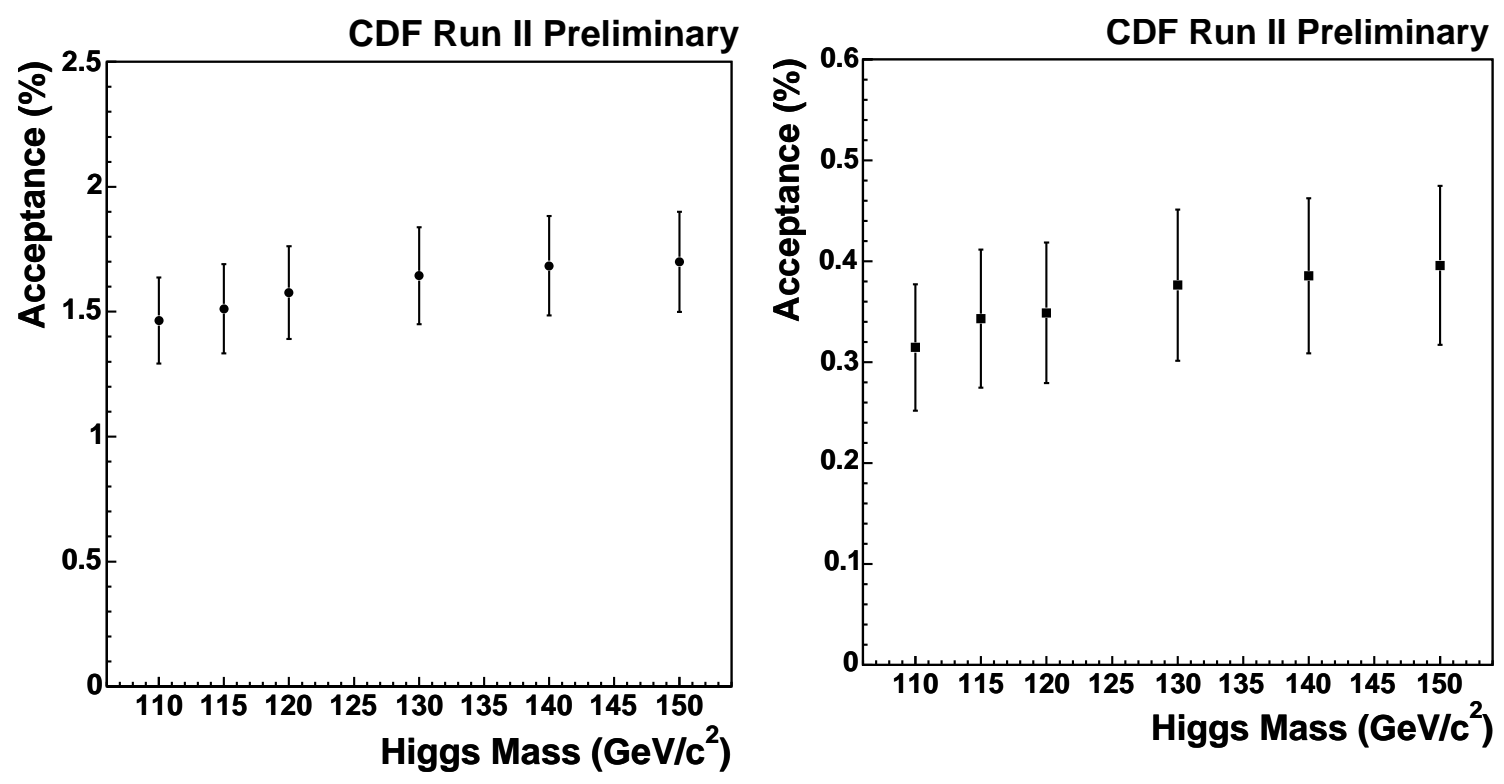

Figure 8.12: The total $W^{ \pm} H \rightarrow W b \bar{b}$ acceptance as a function of the Higgs boson mass for (Left)at least one $b$-tagging method, (Right)double $b$-tagging method. The left plot is the same as Figure 8.4. 


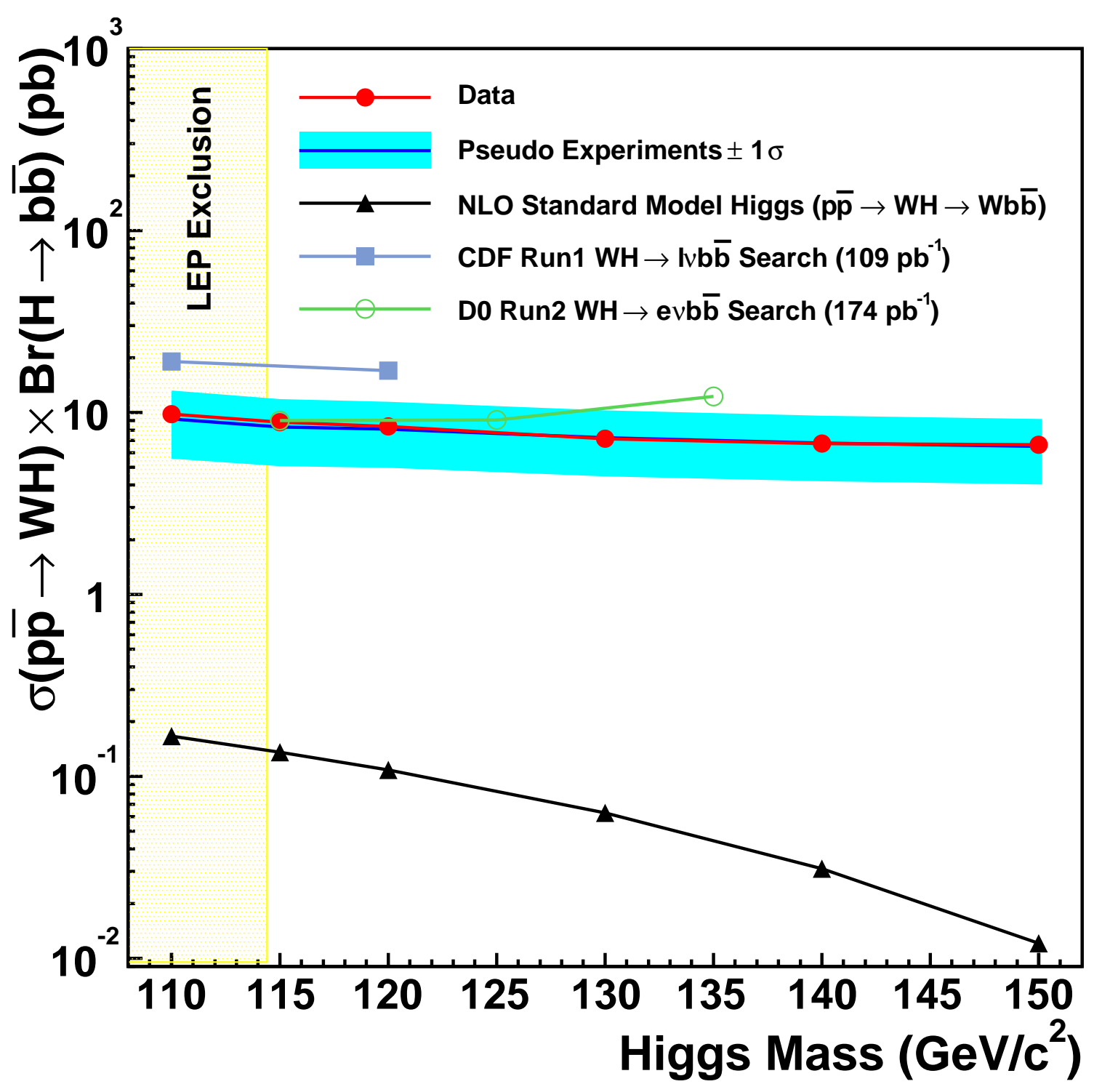

Figure 8.13: The 95\% C.L. upper limits (red circles) on the $W H$ cross section as a function of the Higgs boson mass for double $b$-tagged events. Also shown are the theoretical cross section (black triangles) for Standard Model Higgs boson production in association with a $W$ boson, and the expected limit results from pseudo-experiments (blue region). 


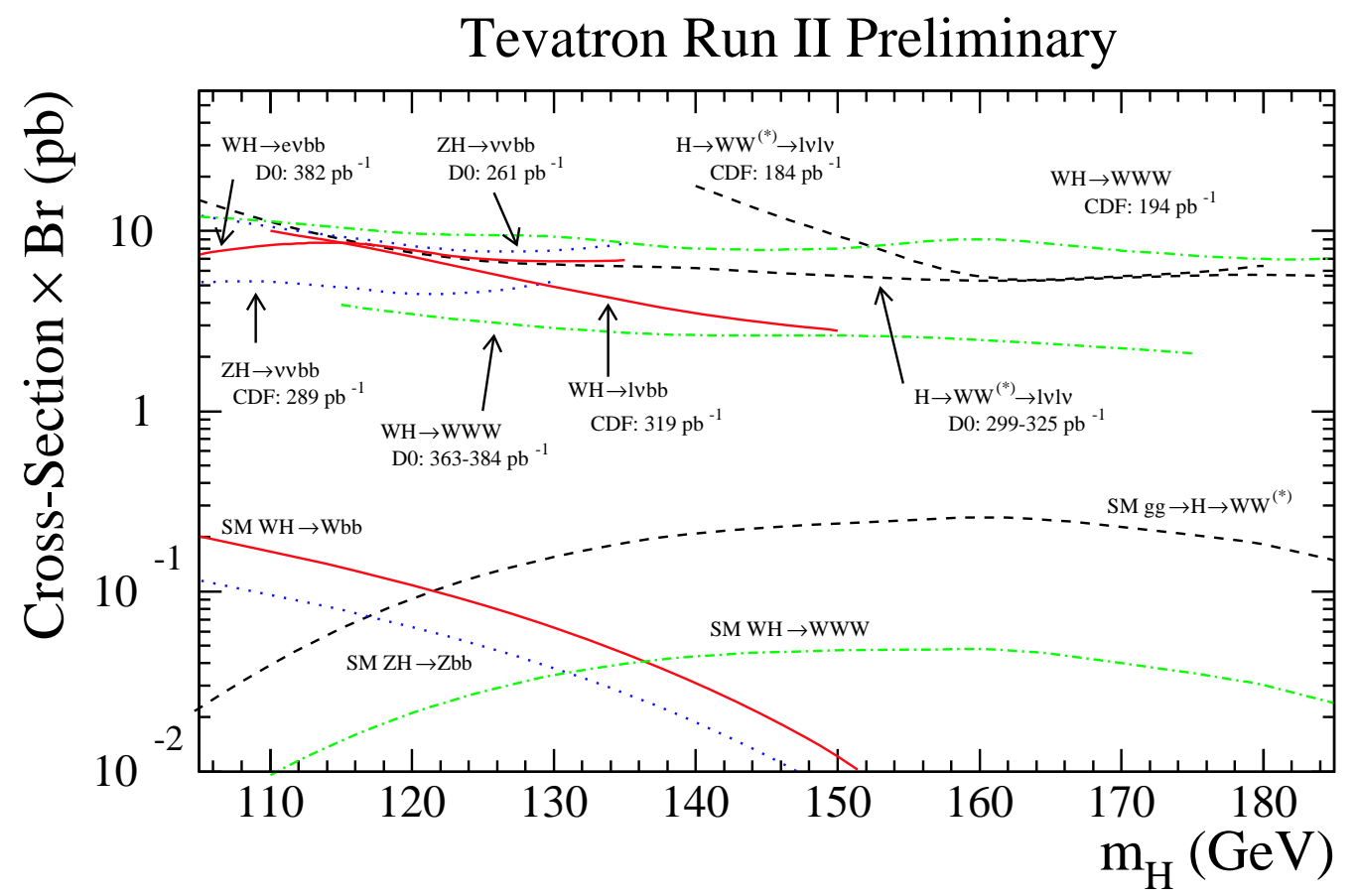

Figure 8.14: The current Higgs boson search results at Tevatron.

for the $95 \%$ C.L. upper limits on the production cross section. For CDF results, $W H \rightarrow \ell \nu b \bar{b}\left(319 \mathrm{pb}^{-1}\right)[72], Z^{0} H \rightarrow \nu \bar{\nu} b \bar{b}\left(289 \mathrm{pb}^{-1}\right)$ [73], $H \rightarrow W^{+} W^{-}(184$ $\left.\mathrm{pb}^{-1}\right)$ [74] and $W H \rightarrow W W^{+} W^{-}\left(194 \mathrm{pb}^{-1}\right)$ [75] are shown. For D $\varnothing$ results, $W H \rightarrow e \nu b \bar{b}\left(382 \mathrm{pb}^{-1}\right)$ [76], $Z^{0} H \rightarrow \nu \bar{\nu} b \bar{b}\left(261 \mathrm{pb}^{-1}\right)$ [77], $H \rightarrow W^{+} W^{-}(325$ $\mathrm{pb}^{-1}$ for $e^{+} e^{-} \nu \bar{\nu}$ channel, $318 \mathrm{pb}^{-1}$ for $e^{ \pm} \mu^{\mp} \nu \bar{\nu}$ channel and $299 \mathrm{pb}^{-1}$ for $\mu^{+} \mu^{-} \nu \bar{\nu}$ channel) [78] and $W H \rightarrow W W^{+} W^{-}\left(384 \mathrm{pb}^{-1}\right.$ for $e^{ \pm} e^{ \pm} \nu \nu$ channel, $368 \mathrm{pb}^{-1}$ for $e^{ \pm} \mu^{ \pm} \nu \nu$ channel and $363 \mathrm{pb}^{-1}$ for $\mu^{ \pm} \mu^{ \pm} \nu \nu$ channel) [79] are shown.

\subsection{Future Prospect}

The Tevatron Higgs Sensitivity Working group estimated the search potential for the Higgs boson in 2000 [80] and 2003 [81]. The studies focus on a number of important improvements including the detectors, $b$-tagging, dijet mass resolution, and the advanced analysis technique such as a neural network. Figure 8.15 shows the Higgs boson search sensitivity as functions of the Higgs boson mass and the integrated luminosity, where the curves shown are obtained by combining the $\ell \nu b \bar{b}$, $\nu \bar{\nu} b \bar{b}$ and $\ell^{+} \ell^{-} b \bar{b}$ channels in the low mass region $\left(90<m_{H}<130 \mathrm{GeV} / c^{2}\right)$, and the $\ell^{ \pm} \ell^{ \pm} j j \nu \nu$ and $\ell^{+} \ell^{-} \nu \bar{\nu}$ channels in the high mass region $\left(130<m_{H}<200 \mathrm{GeV} / c^{2}\right)$. The understanding of the Higgs boson sensitivity will improve over time, as we get more data, with a better understood detector and more clever ideas. Finding the Higgs boson at the Tevatron will be challenging. With $5 \mathrm{fb}^{-1}$, if the Higgs boson does exist, the Tevatron expects to exclude the Standard Model Higgs boson up to $130 \mathrm{GeV} / c^{2}$ at $95 \%$ C.L. or have $3 \sigma$ evidence for the Higgs boson up to 115 


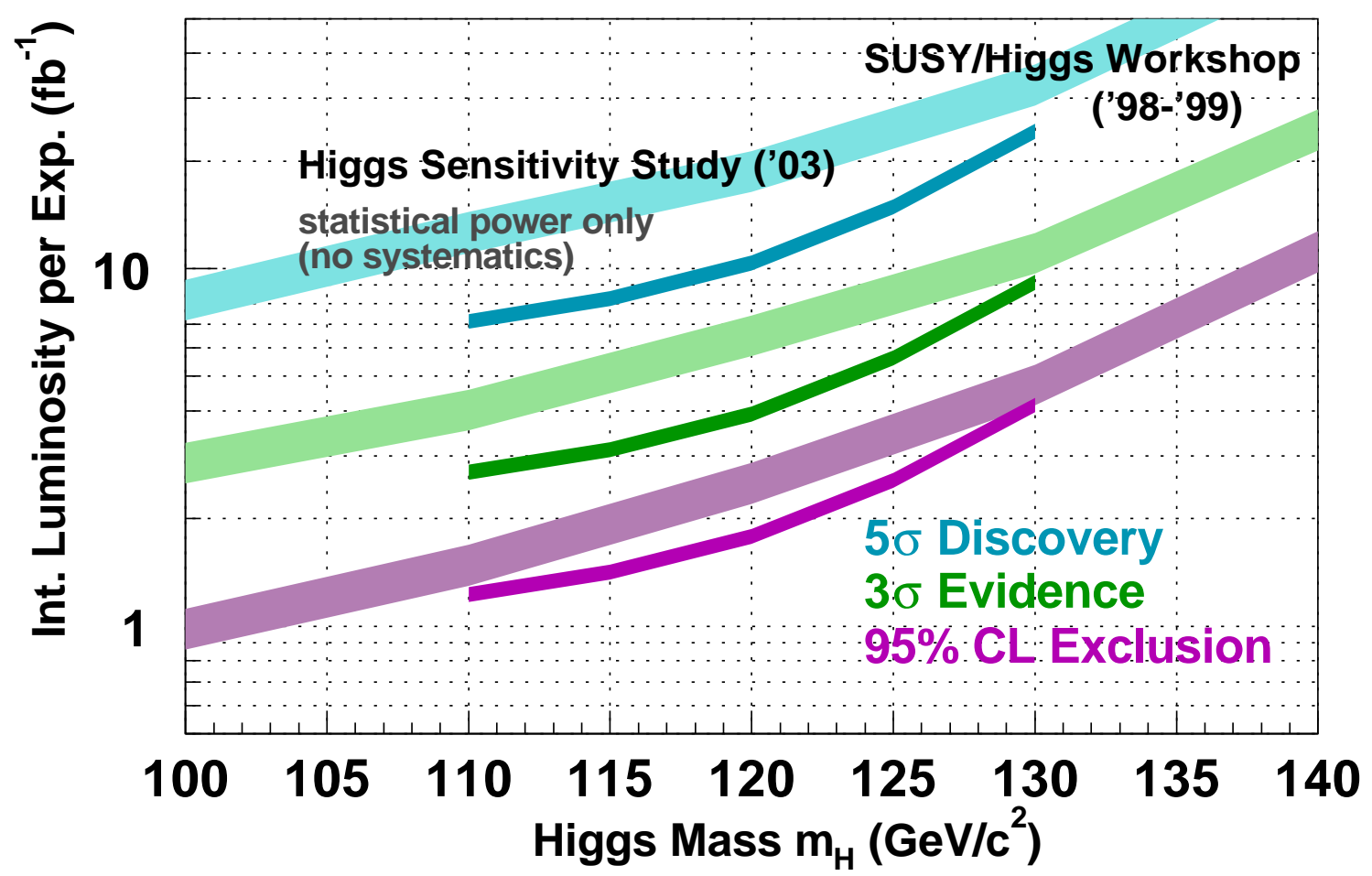

Figure 8.15: The Higgs boson search sensitivity at Tevatron.

$\mathrm{GeV} / c^{2}$. 


\section{Chapter 9}

\section{Conclusion}

We have conducted a search for $W H \rightarrow \ell \nu b \bar{b}$ in $1.96-\mathrm{TeV} p \bar{p}$ collisions using the CDF detector. The data were collected from February 2002 to August 2004. The corresponding integrated luminosity is $319 \mathrm{pb}^{-1}$. We have exploited the $b$-tagged $W+2$ jets events to carry out a search for the Higgs boson decaying into $b \bar{b}$ and produced in association with a $W$ boson. We observed 187 events, which agreed with the Standard Model background expectation of $175.2 \pm 26.3$ events, and there was no significant resonance corresponding to the Higgs boson in the reconstructed dijet invariant mass distribution. As a result, we set a 95\% C.L. upper limit on the production cross section times branching ratio decaying into $b \bar{b}$ as a function of the Higgs boson mass. The results are listed in Table 9.1 for at least one $b$-tagging and double $b$-tagging methods. The sensitivity of the present search is limited by small statistics to one or two orders of magnitude higher than the predicted cross sections.

In the next few years, the Tevatron Collider is still in a unique position to search for the dynamics responsible for electroweak symmetry breaking. We will be able to either see some glimmer of new physics or constrain the Standard Model at an unprecedented level, by using several ten times of data as much as the current data.

\begin{tabular}{ccc}
\hline \hline & \multicolumn{2}{c}{$\sigma(p \bar{p} \rightarrow W H) \times \mathcal{B} r(H \rightarrow b b)(\mathrm{pb})$} \\
\hline$m_{H}\left(\mathrm{GeV} / c^{2}\right)$ & At least one $b$-tagging & Double $b$-tagging \\
\hline 110 & 10.0 & 9.7 \\
115 & 8.6 & 8.8 \\
120 & 7.2 & 8.3 \\
130 & 4.9 & 7.1 \\
140 & 3.5 & 6.7 \\
150 & 2.8 & 6.6 \\
\hline \hline
\end{tabular}

Table 9.1: The 95\% C.L. upper limits for at least one $b$-tagging and double $b$-tagging methods. 



\section{Appendix A}

\section{Non- $W$ Background}

In Section 6.1 and Section 7.3, we estimate the number of events for the non- $W$ background and discuss the dijet invariant mass shapes. However, it would be desirable to understand better the predictions for some specific processes using MC events, such as $b \bar{b}, \gamma+$ jets, $W \rightarrow \tau \nu$ and $Z^{0} \rightarrow \mu^{+} \mu^{-}$.

\section{A.1 $b \bar{b}$}

We have investigated the lepton isolation $I$ versus $\mathbb{E}_{T}$ distributions in large $b \bar{b} \mathrm{MC}$ samples used for the $b$-tagging scale factor measurement [51, 52, 53], where the $2 \rightarrow 2$ process is generated and one lepton with $p_{T}>8 \mathrm{GeV}$ is required at the generator level. The sample is equivalent to a total integrated luminosity of $\sim 170$ $\mathrm{pb}^{-1}$. There is no evidence of any bias on the $b$-tagging rate versus $I$ and $\mathbb{E}_{T}$, shown in Figure A.1, which assures the use of sidebands to extrapolate to the signal region [55].

We have also checked the $I$ versus $\mathbb{E}_{T}$ method for these $b \bar{b}$ events and $\pi^{0} \rightarrow e^{+} e^{-} \gamma$ events with 1 and 2jets, shown in Table A.1, where the $\mathscr{E}_{T}$ cut is lowered to $15 \mathrm{GeV}$ in order to improve the statistics in the signal region. Within the statistics, the $I$ versus $\mathbb{E}_{T}$ method seems to work for both $b \bar{b}$ and fake electrons from $\pi^{0}$ decay. Figure A.2 shows the corresponding dijet and $W$ transverse mass distributions for events passing the standard selection.

\section{A.2 $\gamma+$ Jets}

One of the non- $W$ backgrounds in the electron channel is from $\gamma+$ jets events where the $\gamma$ converts into a $e^{+} e^{-}$pair. Since the $\gamma$ is likely to be isolated, there is some concern that the $I$ versus $\mathbb{E}_{T}$ method may not work for this particular process. We looked at the $\gamma+$ jets MC events made by the $\gamma+$ jets group; this dataset corresponds to about $187 \mathrm{pb}^{-1}$. The photon is required to have $p_{T}$ above $22 \mathrm{GeV} / c$ at the generator level. After the standard selection, we found 31 pretag events and 2 tagged events in $W+$ jets. These give 53.6 events and 3.5 events expected in the pretag and tagged sample for $319 \mathrm{pb}^{-1}$ data, which is about $2 \%$ of the total sample. 


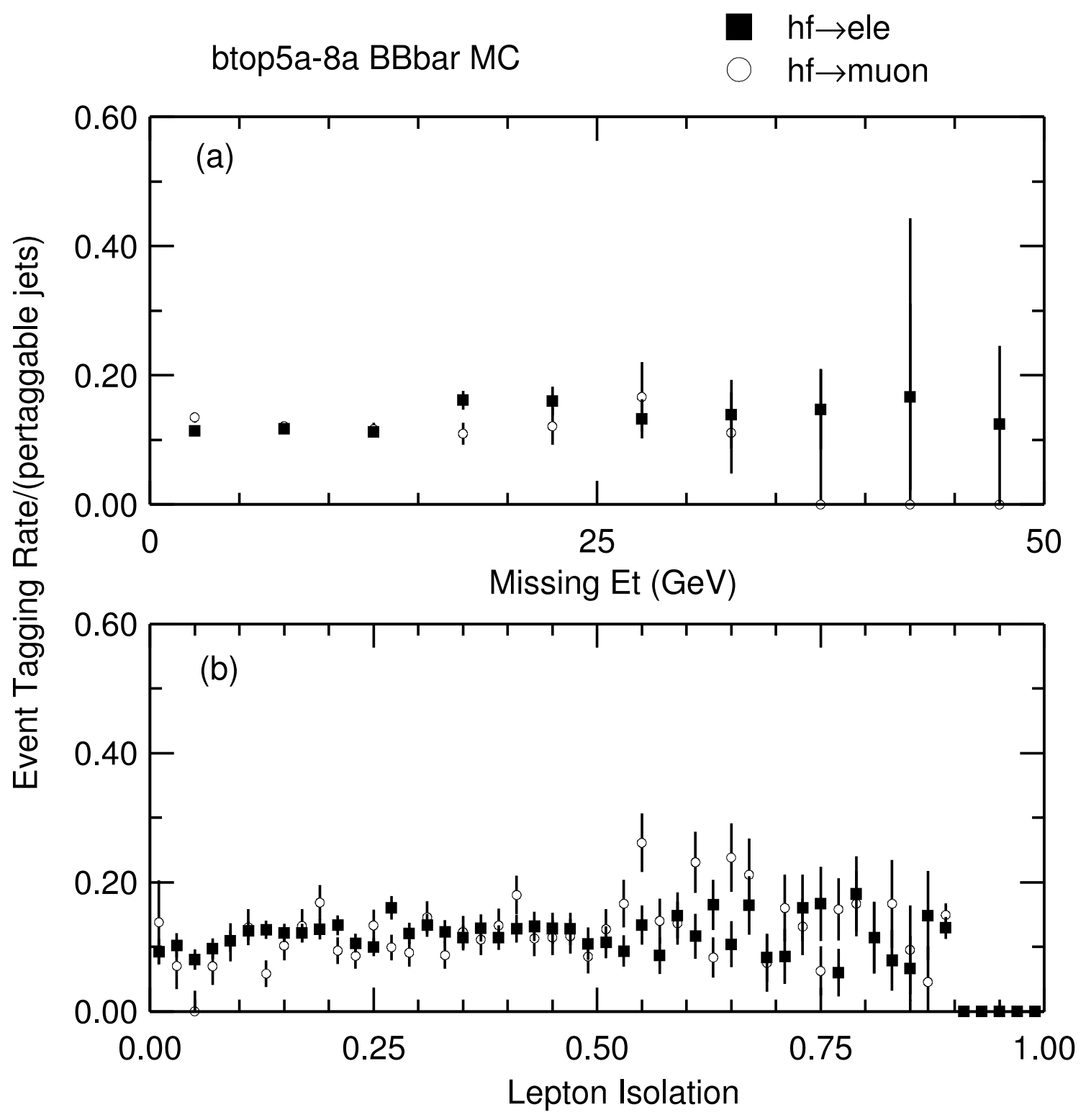

Figure A.1: The $b$-tagging rates in $b \bar{b}$ decays as functions of (a) $\mathbb{E}_{T}$ and (b)lepton isolation. 
(a) Electron

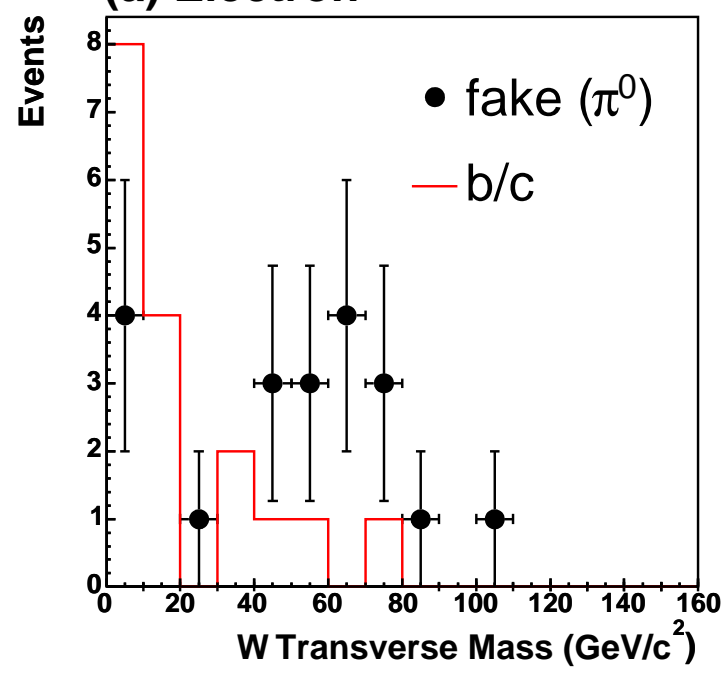

(a) Electron

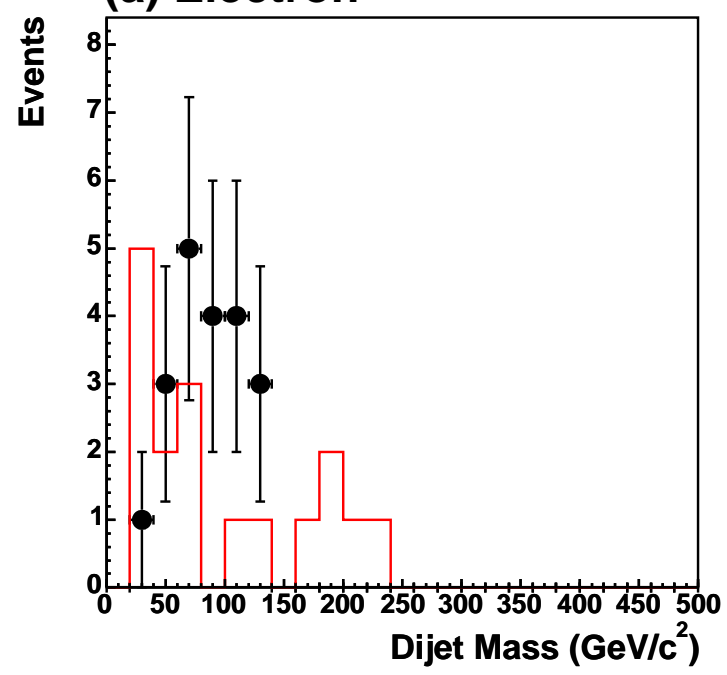

(b) Muon

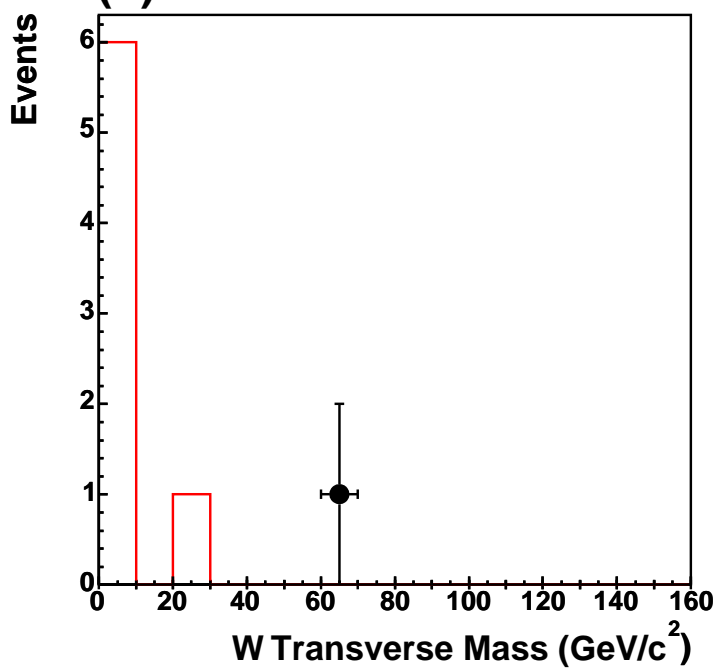

(b) Muon

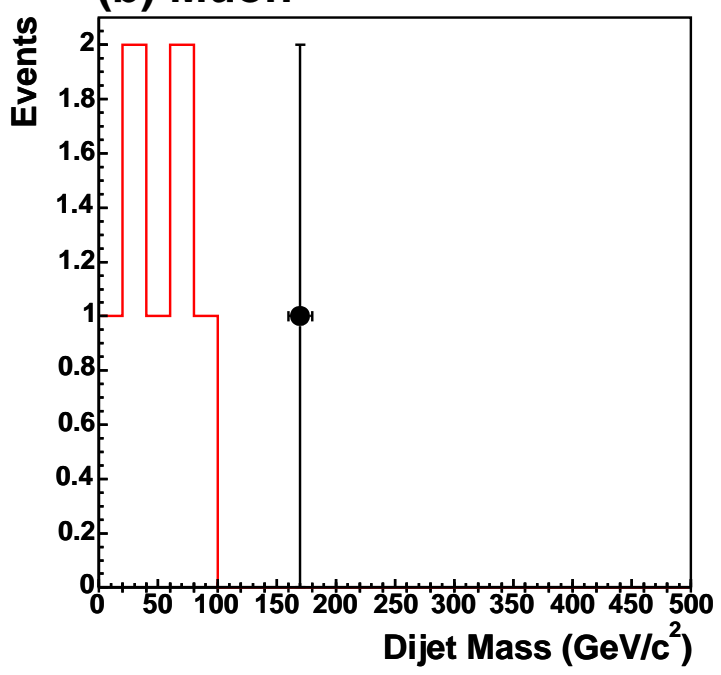

Figure A.2: The dijet and $W$ transverse mass distributions from $b \bar{b}$ and fake $\left(\pi^{0}\right.$ decays) samples after the standard selection. (Top) $W$ transverse mass, (Bottom)dijet mass, (Left)electron channel, (Right)muon channel. 


\begin{tabular}{c|ccc|cc}
\hline \hline & \multicolumn{3}{|c|}{ Pretag } & \multicolumn{2}{c}{ Tagged } \\
\hline Regions & $e(b b)$ & $e\left(\pi^{0}\right)$ & $\mu(b b)$ & $e(b b)$ & $\mu(b b)$ \\
\hline $\mathrm{A}\left(I>0.2\right.$ and $\left.\mathbb{E}_{T}<15 \mathrm{GeV}\right)$ & 6636 & 1668 & 4348 & 678 & 491 \\
$\mathrm{~B}\left(I<0.1\right.$ and $\left.\mathbb{E}_{T}<15 \mathrm{GeV}\right)$ & 1683 & 1448 & 410 & 124 & 23 \\
$\mathrm{C}\left(I>0.2\right.$ and $\left.\mathbb{E}_{T}>15 \mathrm{GeV}\right)$ & 819 & 155 & 503 & 116 & 54 \\
$\mathrm{D}\left(I<0.1\right.$ and $\left.\mathbb{E}_{T}>15 \mathrm{GeV}\right)$ & 153 & 152 & 34 & 21 & 3 \\
\hline $\mathrm{G}=\left(N_{\mathrm{B}} \cdot N_{\mathrm{C}}\right) /\left(N_{\mathrm{A}} \cdot N_{\mathrm{D}}\right)$ & $1.4 \pm 0.2$ & $0.9 \pm 0.1$ & $1.4 \pm 0.3$ & $1.0 \pm 0.3$ & $0.8 \pm 0.5$ \\
\hline \hline
\end{tabular}

Table A.1: The ratio of predicted and observed non- $W$ background in "signal" region for $b \bar{b}$ and "fake" (electrons from $\pi^{0}$ decay) samples.

\begin{tabular}{lc}
\hline \hline & Ratio \\
\hline$N_{\mathrm{D}} / N_{\mathrm{B}}$ & $0.103 \pm 0.006$ \\
$N_{\mathrm{C}} / N_{\mathrm{A}}$ & $0.112 \pm 0.023$ \\
\hline \hline
\end{tabular}

Table A.2: The ratio of the number of events between Region D (C) and Region B (A) for $\gamma+$ jets sample.

Figure A.3 shows some kinematic distributions from the pretag $\gamma+$ jets sample. Furthermore, we have checked the $I$ versus $\mathbb{E}_{T}$ method using the electron +1 jet events, where the $\mathbb{E}_{T}$ cut is lowered to $15 \mathrm{GeV}$ in order to improve the statistics. Table A.2 shows the ratio of the number of events between Region D (C) and Region $\mathrm{B}(\mathrm{A})$. It gives a consistent prediction of non- $W$ events in the signal region. As the results, we confirm that the $\gamma+$ jets events contribute little to the overall sample.

\section{A.3 $W(\rightarrow \tau \nu)+$ Jets and $Z^{0}\left(\rightarrow \mu^{+} \mu^{-}\right)+$Jets}

There is also some concern that $W(\rightarrow \tau \nu)+$ jets and $Z^{0}\left(\rightarrow \mu^{+} \mu^{-}\right)+$jets events may have very different kinematic distributions from other events, once they pass the standard event selections. In order to see their kinematic distributions independently, we have estimated their contribution in the signal region using MC events. The expected relative contribution to the total sample is about $4.5 \%$ for $W \rightarrow \tau \nu$ and about $2 \%$ for $Z^{0} \rightarrow \mu^{+} \mu^{-}$. Figure A.4 shows the dijet mass and $W$ transverse mass distributions in these two physics processes for comparison. The effect of different shape is discussed in Appendix A.4. 

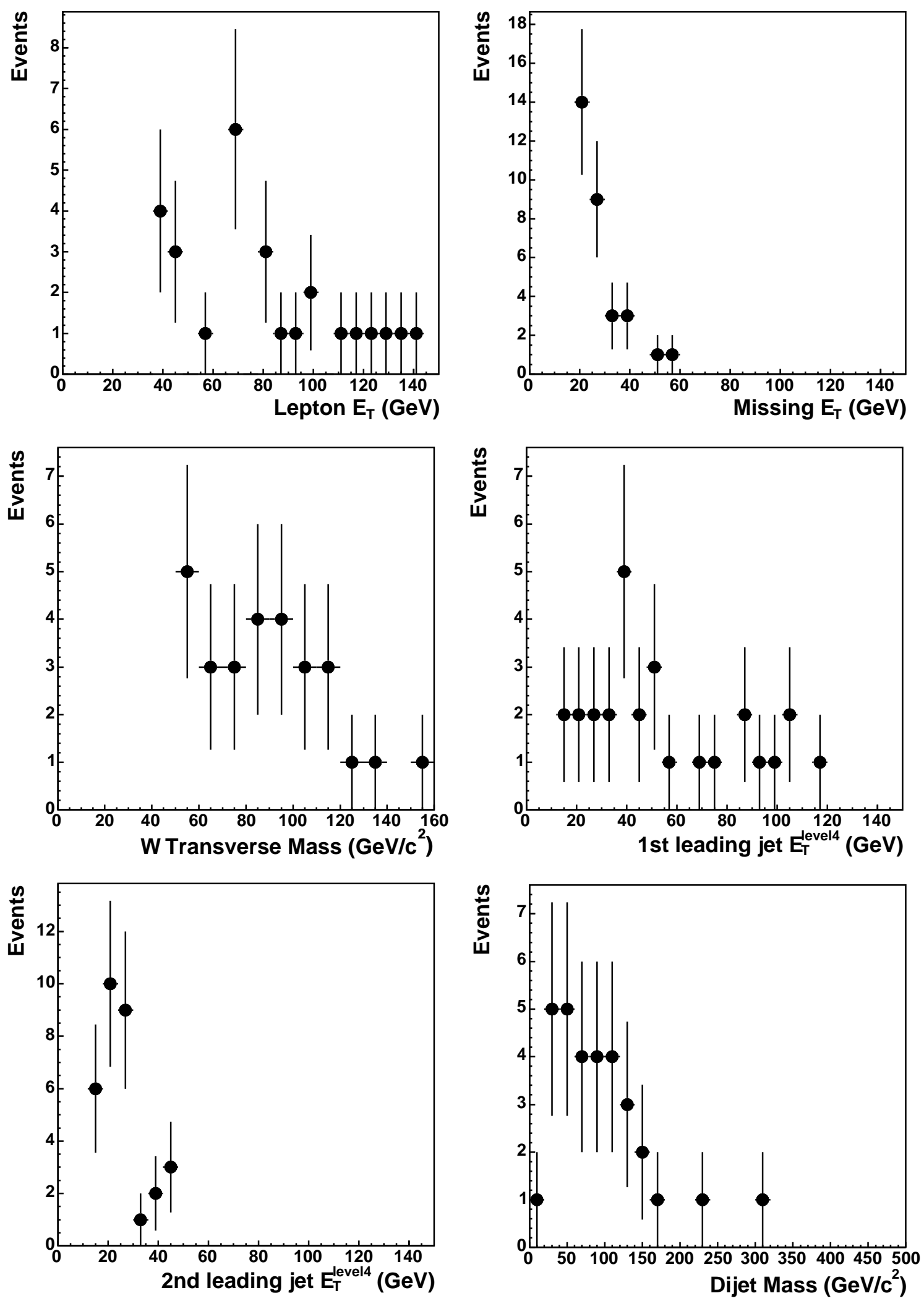

Figure A.3: Some kinematic distributions for pretag $\gamma+$ jets events. (Upper left)Lepton $E_{T}$, (Upper right) $E_{T}$, (Middle left) $W$ transverse mass, (Middle right)1st leading jet $E_{T}$, (Lower left)2nd leading jet $E_{T}$, (Lower right)dijet mass. 

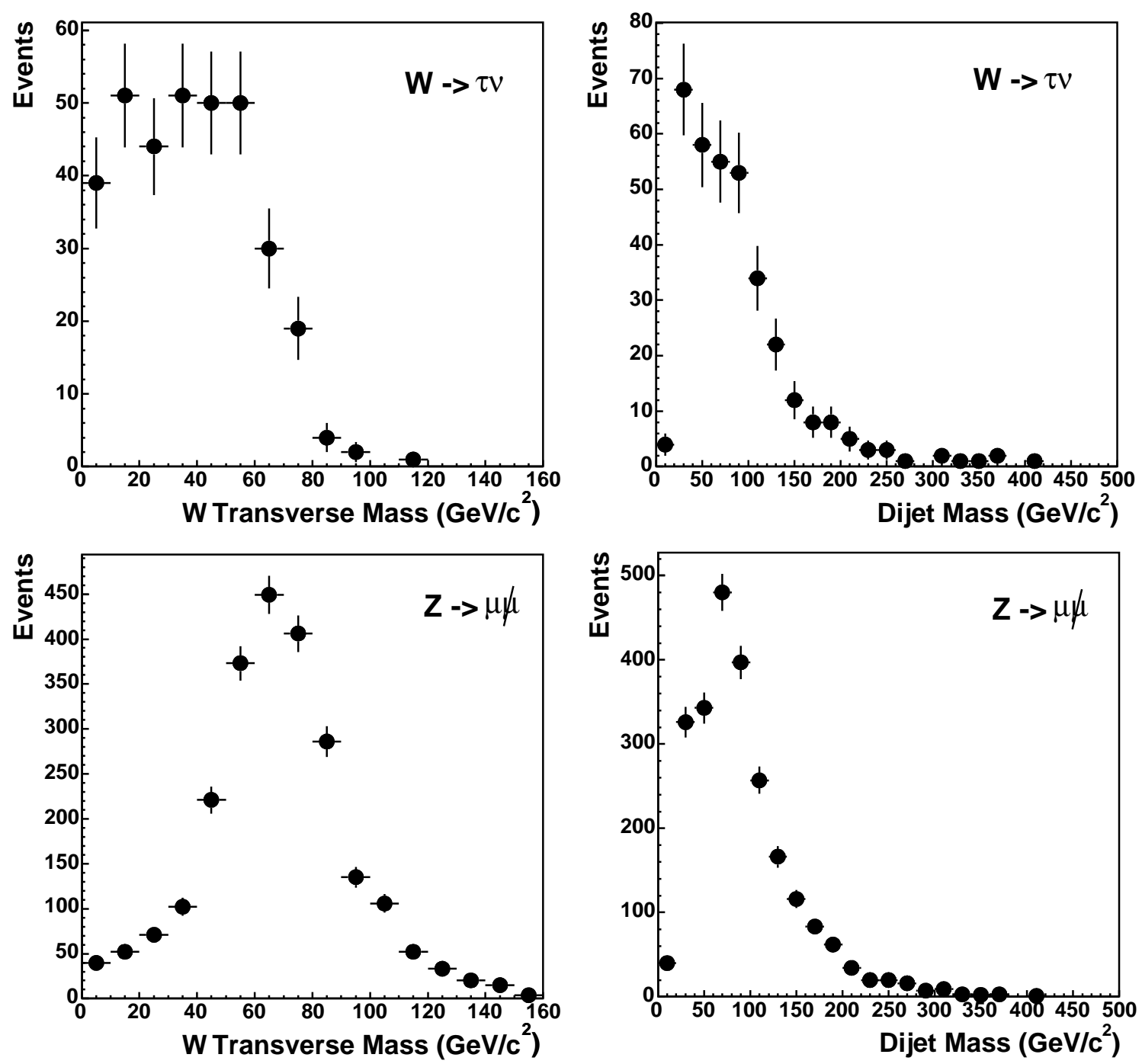

Figure A.4: The distributions of dijet and $W$ transverse mass from $W \rightarrow \tau \nu$ and $Z^{0} \rightarrow \mu^{+} \mu^{-}$. (Top) $W \rightarrow \tau \nu$ sample, (Bottom) $Z^{0} \rightarrow \mu^{+} \mu^{-}$sample, (Left) $W$ transverse mass, (Right)dijet mass. 


\section{A.4 Dijet Mass and $W$ Transverse Mass Distri- butions}

After checking carefully each source of non- $W$ events, we do not find anything significantly wrong in our treatment of non- $W$ background. Figure A.5 shows the comparison of the $W$ transverse mass distributions from data, the expected nominal shape and the alternative shape that includes $W \rightarrow \tau \nu$ and $Z^{0} \rightarrow \mu^{+} \mu^{-}$properly. The distributions are normalized to the same number of data events. The alternative shape seems to describe the data slightly better in the low $W$ transverse region than the shape we previously used; however, the effect is not significant taking into account the limited data statistics.

\section{(a) Electron}

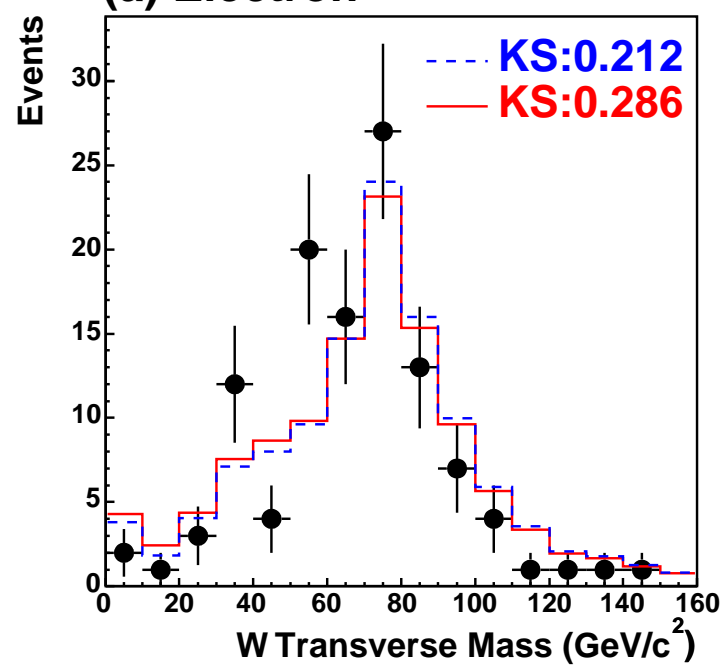

(c) Total

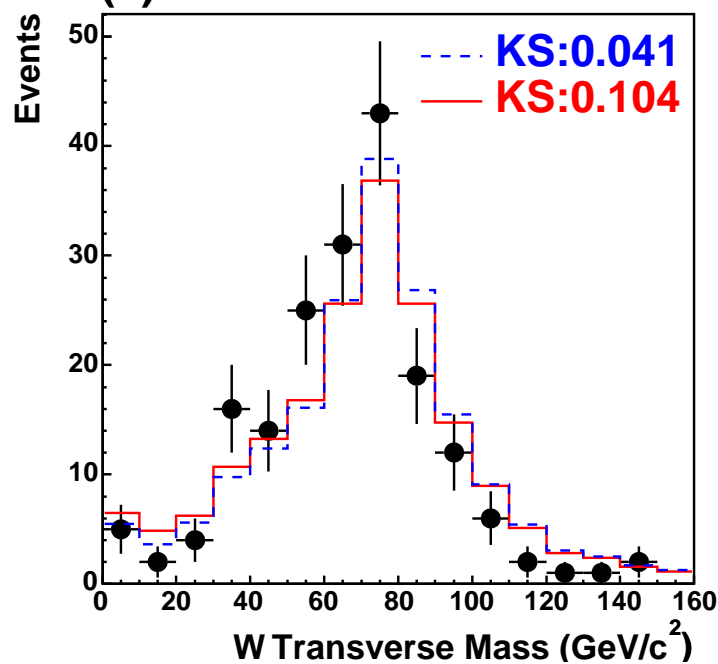

(b) Muon

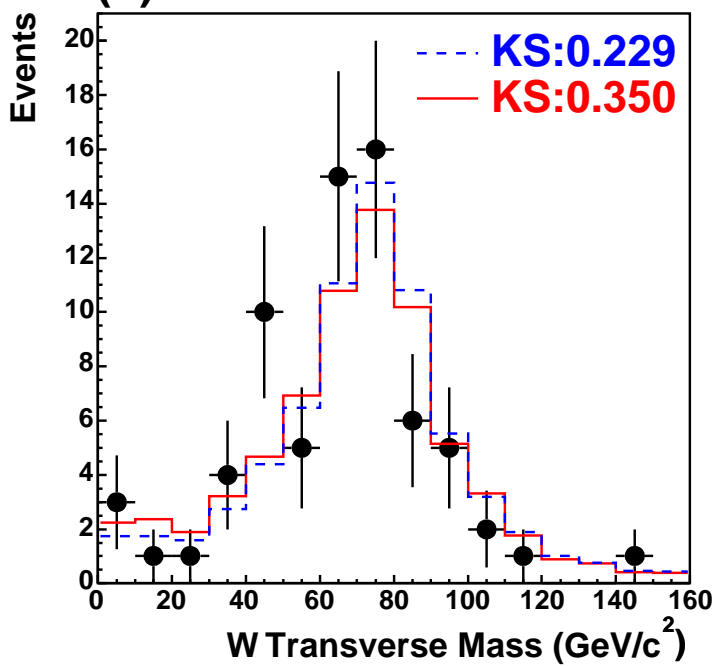

Figure A.5: The comparison of $W$ transverse mass in data and expected shape (see text). (a)electron channel, (b)muon channel, (c)electron + muon channel. 
Figure A.6 shows the comparison of the dijet mass distributions. The alternative shape is in good agreement with others, and the effect is not significant again.

(a) Electron

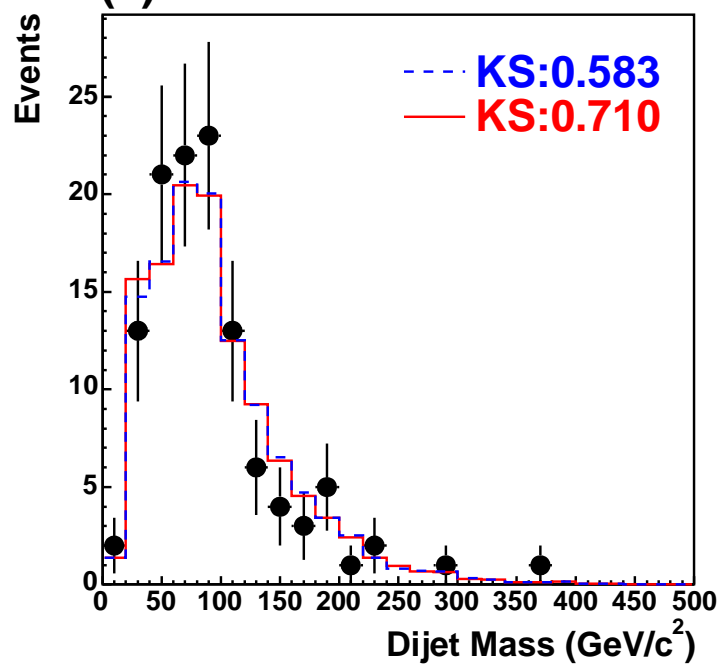

(c) Total

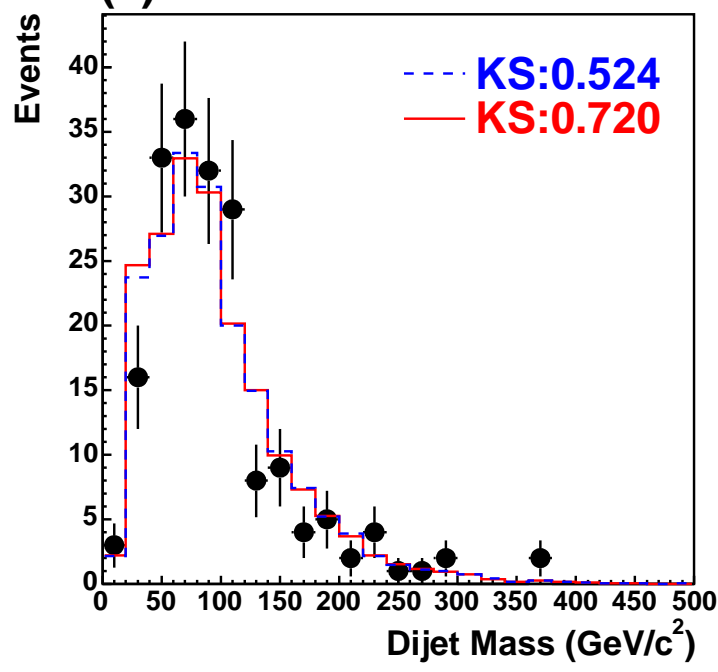

(b) Muon

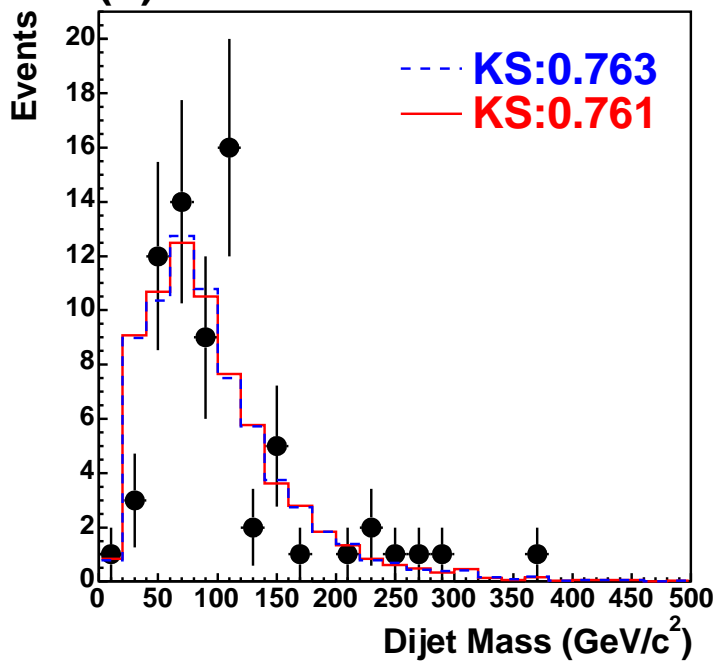

Figure A.6: The comparison of dijet mass in data and expected shape (see text). (a)electron channel, (b)muon channel, (c)electron + muon channel.

\section{A.5 A Cross Check with Additional Cut on Non- $W$}

As a cross check, we have repeated the analysis using an additional cut to reduce the non- $W$ background [82]. The cut requires in addition $0.5<\Delta \phi<2.6$ between the leading jet and $\mathbb{E}_{T}$ directions for events with $\mathbb{E}_{T}<30 \mathrm{GeV}$. This cut, developed 


\begin{tabular}{ccc}
\hline \hline Backgrounds & $\mathrm{w} / \mathrm{o} \Delta \phi$ & $\mathrm{w} / \Delta \phi$ \\
\hline Events before tagging & 3910 & 3378 \\
\hline Mistags & $39.3 \pm 3.1$ & $35.1 \pm 2.9$ \\
$W+b \bar{b}$ & $54.0 \pm 18.4$ & $48.6 \pm 16.5$ \\
$W+c \bar{c}$ & $19.5 \pm 6.6$ & $17.5 \pm 6.0$ \\
$W+c$ & $16.8 \pm 4.3$ & $15.1 \pm 3.9$ \\
Diboson $/ Z^{0} \rightarrow \tau^{+} \tau^{-}$ & $5.0 \pm 1.1$ & $4.6 \pm 1.0$ \\
non- $W$ & $16.5 \pm 3.2$ & $9.9 \pm 2.0$ \\
$t \bar{t}$ & $14.1 \pm 2.5$ & $14.0 \pm 2.4$ \\
single top & $9.6 \pm 2.0$ & $9.1 \pm 1.9$ \\
\hline Total & $174.7 \pm 26.3$ & $153.8 \pm 23.6$ \\
\hline Observed tags & 187 & 168 \\
\hline \hline
\end{tabular}

Table A.3: The comparison of the backgrounds and observed number of tags with and without $\Delta \phi$ cut.

in the top group for the cross section measurement, reduces the signal efficiency about $5 \%$ and the non- $W$ background by $50 \%$. Since the non- $W$ background is a small part of the total tagged background, the cut does not improve the search limit judged based on pseudo-experiment studies. However, it is interesting to check the robustness of our results against such a cut. Figure A.7 shows very similar dijet mass distributions with and without additional $\Delta \phi$ cut. The comparisons of data and expected background events are shown in Table A.3. The numbers of events are consistent with the observed events for both methods.
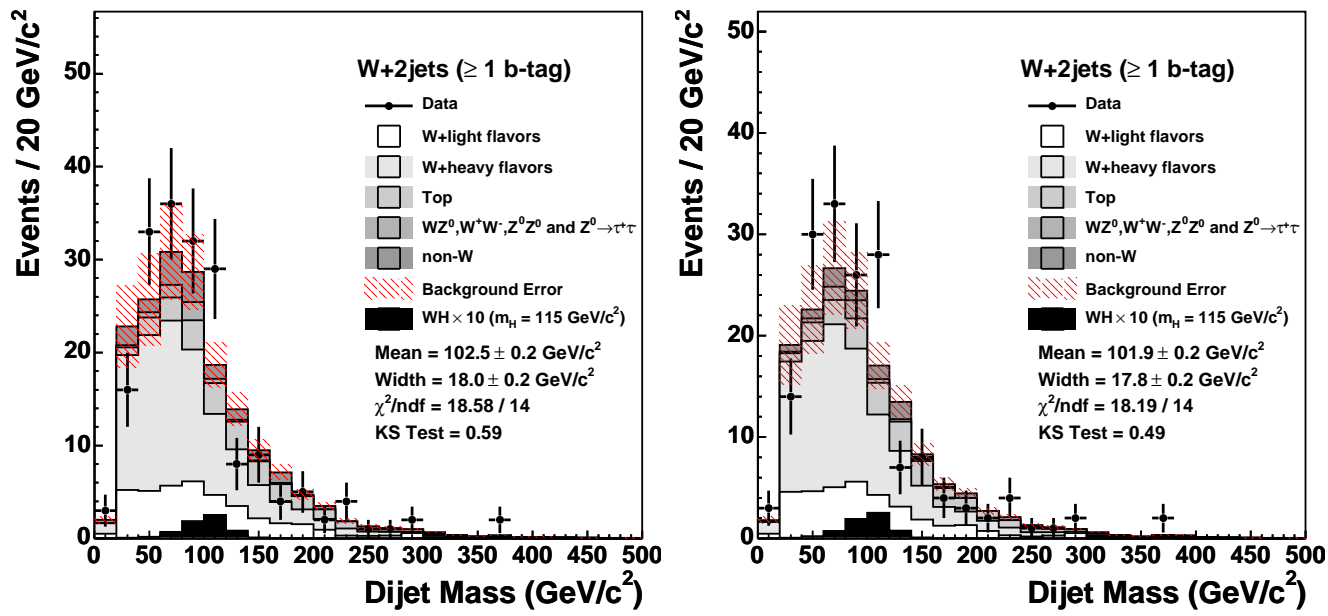

Figure A.7: The comparisons of dijet mass distribution from data and expected background with (left) and without (right) the $\Delta \phi$ cut. The left plot is the same as Figure 7.27. 



\section{Appendix B}

\section{Kolmogorov-Smirnov Test}

The Kolmogorov-Smirnov Test (or KS Test for short) [83] is often applied to see if two distributions are different; that is, to see if we can disprove the null hypothesis that they are drawn from the same population distribution function.

In the KS Test the two distributions to be compared are first converted into two cumulative probability distributions. Different distribution functions give different cumulative probability distributions. They all agree at the end points, however, That is, at the smallest value of $x$ every cumulative distribution is zero; and at the largest value of $x$ it is always unity. In other words, it is the behavior between these two end points that distinguishes distributions. What makes the KS Test ideal for comparing (low statistics) empirical distributions and theoretical curves is that it is applicable to unbinned distributions.

The Kolmogorov-Smirnov distance $D$ is a simple statistic to measure the overall difference between two cumulative distributions: It is defined as the maximum value of the absolute difference between two cumulative distributions. Thus, for comparing one dataset to a known distribution, KS statistic is

$$
D=\max _{-\infty<x<+\infty}\left|S_{N}(x)-P(x)\right|
$$

where $S_{N}(x)$ and $P(x)$ denote the cumulative probability distribution of the dataset and that of the known distribution.

The value $D$ is essentially a random variable but its distribution, when used in the context of null hypothesis, has been well studied. For large $N$, Kolmogorov found the limit distribution:

$$
Q_{K S}(\lambda)=2 \sum_{i=1}^{\infty}(-1)^{i-1} e^{-2 i^{2} \lambda^{2}},
$$

where $\lambda=\sqrt{N} D$. Given $\lambda$, this function returns the probability that the two distributions are compatible. In practice, $N=10 \sim 20$ is large enough. 



\section{Bibliography}

[1] S. L. Glashow, Nucl. Phys. 20, 579 (1961);

S. Weinberg, Phys. Rev. Lett. 19, 1264 (1967).

[2] F. Abe et al., Phys. Rev. Lett. 74, 2626 (1995).

[3] K. Kodama et al., Phys. Lett. B 504, 218 (2001).

[4] P. W. Higgs, Phys. Rev. Lett. 12, 132 (1964);

P. W. Higgs, Phys. Rev. 145, 1156 (1996).

[5] ALEPH, DELPHI, L3 and OPAL Collaborations and the LEP Working Group for Higgs Boson Searches, Phys. Lett. B 565, 61 (2003).

[6] ALEPH, DELPHI, L3 and OPAL Collaborations, the LEP Electroweak Working Group and the SLD Electroweak and Heavy Flavor Groups, arXiv:hep-ex/0312023;

The LEP Electroweak Working Group, http://lepewwg. web.cern.ch/LEPEWWG/.

[7] S. Weinberg, Phys. Rev. Lett. 19, 1264 (1976).

[8] The Gargamelle Neutrino Collaboration, F. J. Hasert et al., Phys. Lett. B 46, 138 (1973).

[9] G. Arnison et al., Phys. Lett. B 122, 103 (1983);

G. Arnison et al., Phys. Lett. B 126, 398 (1983).

[10] S. Eidelman et al., Phys. Lett. B 592, 1 (2004).

[11] M. Kobayashi and T. Maskawa, Prog. Theor. Phys. 49, 652 (1973).

[12] Michael Spira and Peter M. Zerwas, CERN-TH/97-379.

[13] S. Dawson, BNL-HET-99/1.

[14] D. Acosta et al., Submitted to Phys. Rev. Lett., FERMILAB-PUB-05-042-E.

[15] V. M. Abazov, Phys. Rev. Lett. 94, 091802 (2005).

[16] The CDF Collaboration, FERMILAB-Pub-96/390-E.

[17] C. S. Hill, Nucl. Instrum. Methods A530, 1 (2004). 
[18] A. Sill, Nucl. Instrum. Methods A447, 1 (2000).

[19] A. Affolder et al., Nucl. Instrum. Methods A453, 84 (2000).

[20] T. Affolder et al., Nucl. Instrum. Methods A526, 249 (2004).

[21] L. Balka et al., Nucl. Instrum. Methods A267, 272 (1988).

[22] S. Bertolucci et al., Nucl. Instrum. Methods A267, 301 (1988).

[23] M. Albrow et al., Nucl. Instrum. Methods A431, 101 (1999).

[24] M. Albrow et al., Nucl. Instrum. Methods A480, 524 (2002).

[25] Pawel de Barbaro, FERMILAB-CONF-98/057-E.

[26] G. Apollinari et al., Nucl. Instrum. Methods A267, 301 (1988).

[27] G. Apollinari et al., Nucl. Instrum. Methods A412, 515 (1998).

[28] A. Artikov et al., Submitted to Nucl. Instrum. Methods.

[29] D. Acosta et al., Nucl. Instrum. Methods A461, 540 (2001).

[30] P. D. B. Collins, Cambridge University Press (1977).

[31] S. Klimenko et al., Fermilab-FN-0741.

[32] T. Sjöstrand et al., Comp. Phys. Commun. 135, 238 (2001).

[33] G. Marchesini and B. Webber, Nucl. Phys. B 310, 461 (1998).

[34] G. Corcella et al., JHEP 0101, 010 (2001).

[35] M. Mangano, M. Moretti, F. Piccinini, R. Pittau and A. Polosa, JHEP 07, 001 (2003).

[36] J. Pumplin et al., JHEP 0207, 012 (2002).

[37] P. Avery, K. Read and G. Trahern (1985), unpublished.

[38] R. Brun and F. Carminati, CERN Programing Library Long Writeup W5013 (1993).

[39] G. Grindhammer, M. Rudowicz and S. Peters, Nucl. Instrum. Methods A290, 469 (1990).

[40] T. Affolder et al., Nucl. Instrum. Methods A526, 249 (2004).

[41] Chris Hays, Peter Tamburello, Ashutosh Kotwal, Peter Wittich and Rick Snider, CDF/DOC/TRACKING/CDFR/6992. 
[42] C. Chen, I. Cho, C. Hays, M. Herndon, J. Kraus, J. Kroll, T. Miao, P. Murat, R. OldeMan and J.C Yun, CDF/PHYS/BOTTOM/CDFR/6394.

[43] K. Bloom and W.M. Yao, CDF/DOC/TRACKING/CDFR/5991.

[44] TOP GROUP, $\mathrm{CDF} / \mathrm{ANAL} / \mathrm{TOP} / \mathrm{CDFR} / 2966$.

[45] F. Abe et al., Phys. Rev. D 50, 2966 (1994).

[46] F. Abe et al., Phys. Rev. Lett. 68, 1104 (1992).

[47] Anwar Bhatti and Florencia Canelli, $\mathrm{CDF} / \mathrm{PUB} / \mathrm{JET} / \mathrm{PUBLIC} / 7543$.

[48] D. Acosta et al., arXiv:hep-ex/0410041.

[49] Kevin Burkett and João Guimarães da Costa, CDF/DOC/SEC_VTX/CDFR/6430.

[50] Daniel Sherman, Sal Rappoccio, and João Guimarães da Costa, CDF/ANAL/SEC_VTX/CDFR/7585.

[51] P. Lujan, H. Bachacou, J. Nielsen, W. Yao and T. Wright, CDF/DOC/SEC_VTX/CDFR/7343.

[52] A. Holloway, D. Sherman, S. Rappoccio, and J. Guimarães da Costa, S. Grinstein, CDF/PHYS/SEC_VTX/CDFR/7445.

[53] H. Bachacou, P. Lujan, J. Nielsen, W.Yao, A. Holloway, D. Sherman, S. Rappoccio, J. Guimarães da Costa, S. Grinstein, T. Wright and S. Levy, CDF/DOC/SEC_VTX/CDFR/7480.

[54] Christopher Neu, Daniel Jeans, João Guimarães, Stan Lai, Stephen Levy, Oscar Gonzalez, Salvatore Rappoccio, Tom Wright and Weiming Yao, CDF/DOC/SEC_VTX/CDFR/7578.

[55] H. Bachacou, J. Nielsen and W. Yao, $\mathrm{CDF} / \mathrm{DOC} / \mathrm{TOP} / \mathrm{CDFR} / 6569$.

[56] Yoshio Ishizawa, Jason Nielsen and Weiming Yao, CDF/ANAL/EXOTIC/CDFR/7677.

[57] João Guimarães da Costa and Salvatore Rappoccio, CDF/PUB/SEC_VTX/PUBLIC/7326. 
[58] Andrew Foland, Melissa Franklin, João Guimarães da Costa, Salvatore Rappoccio and Daniel Sherman, CDF/PHYS/TOP/CDFR/7536.

[59] H. Bachacou, C. Ferretti, J. Nielsen and W. Yao, CDF/ANAL/TOP/CDFR/7007.

[60] J. M. Campbell and R. K. Ellis, Phys. Rev. D 65, 113007 (2002).

[61] D. Acsta, et, al., Phys. Rev. Lett. 94, 091803 (2005).

[62] M. Cacciari, S. Frixione, M. Mangano, P. Nason and G. Ridolfi, arXiv:hepph/0303085.

[63] B. W. H. Laenen, L. Phaf, Z. Sullivan and S. Weinzierl, Phys. Rev. D 66, 054024 (2002).

[64] T. Stelzer, Z. Sullivan and S. Willenbrock, Phys. Rev. D 56, 5919 (2002).

[65] Philip R. Bevington and D. Keith Robinson, A Division of The McGraw-Hill Companies, Data Reduction and Error Analysis For The Physical Sciences.

[66] W. K. Sakumoto, CDF/ANAL/ELECTROWEAK/CDFR/7324.

[67] Yoshio Ishizawa and Jason Nielsen, CDF/DOC/ELECTRON/CDFR/7401.

[68] Victoria Martin, CDF/DOC/MUON/7367.

[69] C. Hill, J. Incandela, and C. Mills, CDF/DOC/ELECTRON/CDFR/7309.

[70] H. Bachacou, M. McFarlane and W. Yao, CDF/ANAL/TOP/CDFR/7682.

[71] Oscar Gonzalez and Carsten Rott, CDF/PHYS/EXOTIC/CDFR/7051.

[72] Y. Ishizawa, J. Nielsen and W. Yao, CDF/PUB/EXOTIC/PUBLIC/7740.

[73] Viktor Veszpremi, Daniela Bortoletto, Art Garfinkel, Oscar Gonzalez and Carsten Rott, CDF/ANAL/EXOTIC/CDFR/7719.

[74] Sunny S. Chuang, D. Carlsmith, S. Cabrera, M. Kruse, D. McGivern and D. Waters, $\mathrm{CDF} / \mathrm{DOC} / \mathrm{EXOTIC} / \mathrm{CDFR} / 6958$. 
[75] Hirokazu Kobayashi, Kazuhiro Yamamoto and Yoshihiro Seiya, CDF/PUB/EXOTIC/PUBLIC/7307.

[76] The DØ Collaboration, DØnote4896-CONF.

[77] The DØ Collaboration, DØnote4774-CONF.

[78] The DØ Collaboration, DØnote4760-CONF.

[79] The DØ Collaboration, DØnote4861-CONF-v0.3.

[80] M. Carena et al., arXiv:hep-ph/0010338.

[81] The CDF and D $\varnothing$ Collaborations, FERMILAB-PUB-03/320-E.

[82] D. Acosta et al., Submitted to Phys. Rev. D, arXiv:hep-ex/0504053.

[83] Brian P. Flannery, Saul A. Teukolsky, and William T. Vetterling, W. H. Press, Numerical Recipes C: The Art of Scientific Computing. 